U.S. Department of the Interior

U.S. Geological Survey

Water-Quality Data for Streams in the Boulder River Watershed, Jefferson County, Montana

By David A. Nimick and Thomas E. Cleasby

Open-File Report 00-99

In cooperation with the

U.S. Department of Agriculture-Forest Service 


\section{U.S. Department of the Interior}

BRUCE BABBITT, Secretary

\section{U.S. Geological Survey}

Charles G. Groat, Director

Any use of trade, product, or firm names in this publication is for descriptive purposes only and does not imply endorsement by the U.S. Government

Helena, Montana

April 2000

For additional information write to:

District Chief

U.S. Geological Survey 3162 Bozeman Avenue

Helena, MT 59601-6456

Copies of this report may be purchased from:

U.S. Geological Survey

Branch of Information Services

Box 25286

Denver, CO 80225-0286 


\section{CONTENTS}

Abstract
Introduction
Purpose and scope
Description of study area
Acknowledgments
Quboratory analysis
Quality-assurance data
Data

\section{ILLUSTRATIONS}

Figure 1-3. Map showing location of:

1. The two watersheds being investigated as pilot studies of the Abandoned Mine Lands Initiative.

2. Water-quality sampling sites in the Boulder River watershed, Montana

3. Selected inactive mines, prospects, and mining-related sites in the Boulder River watershed, Montana.

\section{TABLES}

Table 1. Water-quality sampling sites in the Boulder River watershed, Montana, 1996-99

2. Water-quality data for streams in the Boulder River watershed, Montana, 1996-99

3. Historical water-quality sampling sites in the Boulder River watershed, Montana, with data in the Montana Bureau of Mines and Geology Ground Water Information Center database

4. Historical water-quality data retrieved from the Montana Bureau of Mines and Geology Ground Water Information Center database for streams in the Boulder River watershed, Montana.........

5. Water-quality data for field duplicates, Boulder River watershed, Montana 62

6. Water-quality data for field blanks, Boulder River watershed, Montana 


\section{CONVERSION FACTORS, ABBREVIATED UNITS, AND ACRONYMS}

\begin{tabular}{rll}
\hline Multiply & By & \multicolumn{1}{c}{ To obtain } \\
\hline & & \\
cubic foot per second $\left(\mathrm{ft}^{3} / \mathrm{s}\right)$ & 0.028317 & cubic meter per second \\
foot $(\mathrm{ft})$ & 0.3048 & meter \\
mile $(\mathrm{mi})$ & 1.609 & kilometer \\
\hline
\end{tabular}

Temperature can be converted to degrees Celsius $\left({ }^{\circ} \mathrm{C}\right)$ or degrees Fahrenheit $\left({ }^{\circ} \mathrm{F}\right)$ by the following equations:

$$
\begin{aligned}
& { }^{\circ} \mathrm{C}=5 / 9\left({ }^{\circ} \mathrm{F}-32\right) \\
& { }^{\circ} \mathrm{F}=9 / 5\left({ }^{\circ} \mathrm{C}\right)+32
\end{aligned}
$$

Abbreviated water-quality units used in this report:

$\mu \mathrm{g} / \mathrm{L} \quad$ micrograms per liter

$\mu \mathrm{m} \quad$ micrometer

$\mu \mathrm{S} / \mathrm{cm} \quad$ microsiemens per centimeter at 25 degrees Celsius

$\mathrm{mg} / \mathrm{L} \quad$ milligrams per liter

$\mathrm{mm} \quad$ millimeter

Acronyms used in this report:

MBMG Montana Bureau of Mines and Geology

NWQL National Water-Quality Laboratory of the U.S. Geological Survey

RPD relative percent difference

USDA U.S. Department of Agriculture

USGS U.S. Geological Survey 


\title{
WATER-QUALITY DATA FOR STREAMS IN THE BOULDER RIVER WATERSHED, JEFFERSON COUNTY, MONTANA
}

\author{
By David A. Nimick and Thomas E. Cleasby
}

\begin{abstract}
Chemical data were collected in the Boulder River watershed of southwestern Montana during 1996-99 as part of a detailed interdisciplinary study characterizing the effects of historical inactive mines on streams in the watershed. This report presents water-quality data collected by the U.S. Geological Survey for physical properties, major ions, nutrients, and trace elements for 62 sites in and near the watershed. Supplementary historical water-quality data for 83 sites also are presented.
\end{abstract}

\section{INTRODUCTION}

Trace elements from mineralized areas and abandoned mine lands affect water quality and aquatic biota in many watersheds of the United States. As part of a cooperative effort with Federal land-management agencies, the U.S. Geological Survey (USGS) implemented an Abandoned Mine Lands Initiative in 1996. The goal of the initiative is to develop a strategy for gathering and communicating the scientific information needed to formulate effective and cost-efficient characterization and remediation of the effects of historical mining using a watershed approach (Buxton and others, 1997). The watershed approach is intended to identify and characterize those sites that have the most profound effect on water and ecosystem quality within the watershed. The strategy is based on understanding the fundamental geologic, hydrologic, geochemical, and biologic processes that cause the environmental degradation often observed downstream from historical mining districts. With this understanding, remediation efforts can be designed and conducted effectively by land-management agencies in a focused and costefficient manner. As part of the Abandoned Mine Lands Initiative, pilot studies are being conducted in two watersheds: the Boulder River watershed near
Helena, Montana, and the upper Animas River watershed in southwestern Colorado (fig. 1).

Water is the link connecting toxic trace elements derived from inactive, historical mine sites to aquatic biota and, therefore, is an integral component of the assessment of environmental effects associated with past mining activity. Water quality in the Boulder River Abandoned Mine Lands Initiative study area was characterized by systematic sampling of streams throughout the watershed. Water-quality data from this sampling can be used to characterize current environmental conditions in the watershed and determine the seasonal and spatial distribution of trace element concentrations in streams throughout the watershed. These data also can be used to identify the natural and miningrelated source of those trace elements, understand the transport of dissolved and particulate trace elements, evaluate the potential for metal toxicity to biota, and provide baseline data for future evaluation of the success of clean-up activities at historical mine sites (Nimick and others, 1999).

\section{Purpose and Scope}

The purpose of this report is to present waterquality data for physical properties, major ions, nutrients, and trace elements for water samples collected during 1996-99 in the Boulder River watershed near the town of Basin in Jefferson County, Montana, to support a variety of abandoned mine land studies conducted by the USGS, U.S. Department of Agriculture (USDA-Forest Service), and the Bureau of Land Management. Analytical results are reported for 323 water samples collected by the USGS from 62 sites (fig. 2). Samples were collected at frequencies ranging from one-time site visits to multiple periodic samples collected to determine water-quality conditions over a range of seasonal and annual hydrologic conditions. Quality-assurance data associated with these water- 


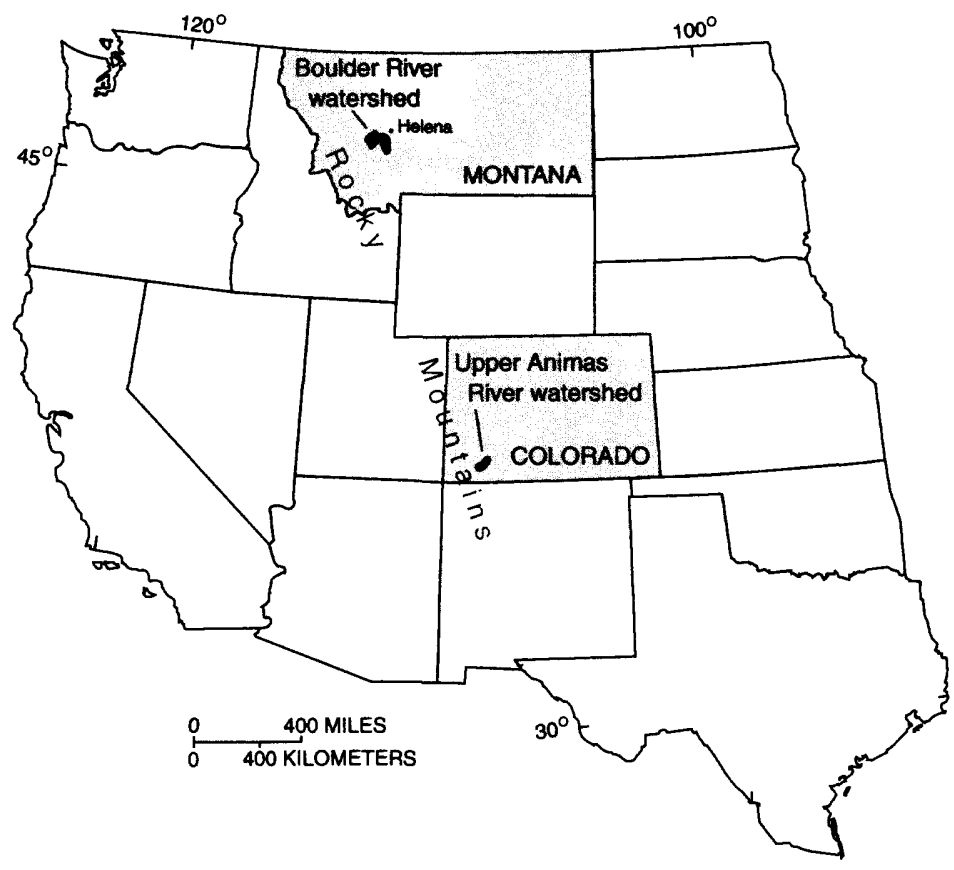

Figure 1. Location of the two watersheds being investigated as pilot studies of the Abandoned Mine Lands Initiative.

quality data are presented. The data-collection efforts were conducted by the USGS in cooperation with the USDA-Forest Service. Historical data from the Montana Bureau of Mines and Geology (MBMG) for selected dissolved trace elements for 83 sites in the watershed (fig. 2) also were compiled and are reported here.

\section{Description of Study Area}

The Boulder River watershed study area includes the drainage areas of three large tributaries (Basin, Cataract, and High Ore Creeks) to the Boulder River and the approximately 9-mile reach of the Boulder River that extends from just upstream (site 3 ) to just downstream (site 58) of these tributaries (fig. 2). The study area does not include the large drainage area of the Boulder River upstream from site 3 or the Boulder River downstream from site 58 to the Jefferson River, even though water quality may be affected by historical mining in this downstream reach. Limited sampling was conducted outside of the study area to document the extent of enriched trace-element concentrations in the Boulder River downstream of the abandoned mine lands and to characterize conditions at locations unaffected by historical mining.

Historical metal-mining activity began in the late 1860s. About 125 inactive mines and prospects are present in the Boulder River watershed. The locations of selected major mines and a smelter are shown on figure 3. Principal metals produced from these mines included silver, gold, lead, zinc, and copper. Mineralization in the Boulder River watershed occurs in quartz veins, and alteration associated with the mineralization is confined to narrow bands around the veins. Ore bodies in the veins, as well as the veins and associated alteration zones, contain sulfide minerals such as pyrite that weather to produce acid and trace elements. The primary effect of mining in the watershed is degraded water quality and aquatic habitat, both of which affect aquatic and fishery resources. Some streams are devoid of fish while others have chronically impaired fisheries (Farag and others, 1999). Inactive mines affect streams through direct discharge of acid drainage from adits, seepage from waste rock and tailings piles, and erosion of mining waste and tailings by storm runoff (Nimick and others, 1999; Farag and others, 1999).

Streamflow in the Boulder River and Basin, Cataract, and High Ore Creeks is typical of mountain 


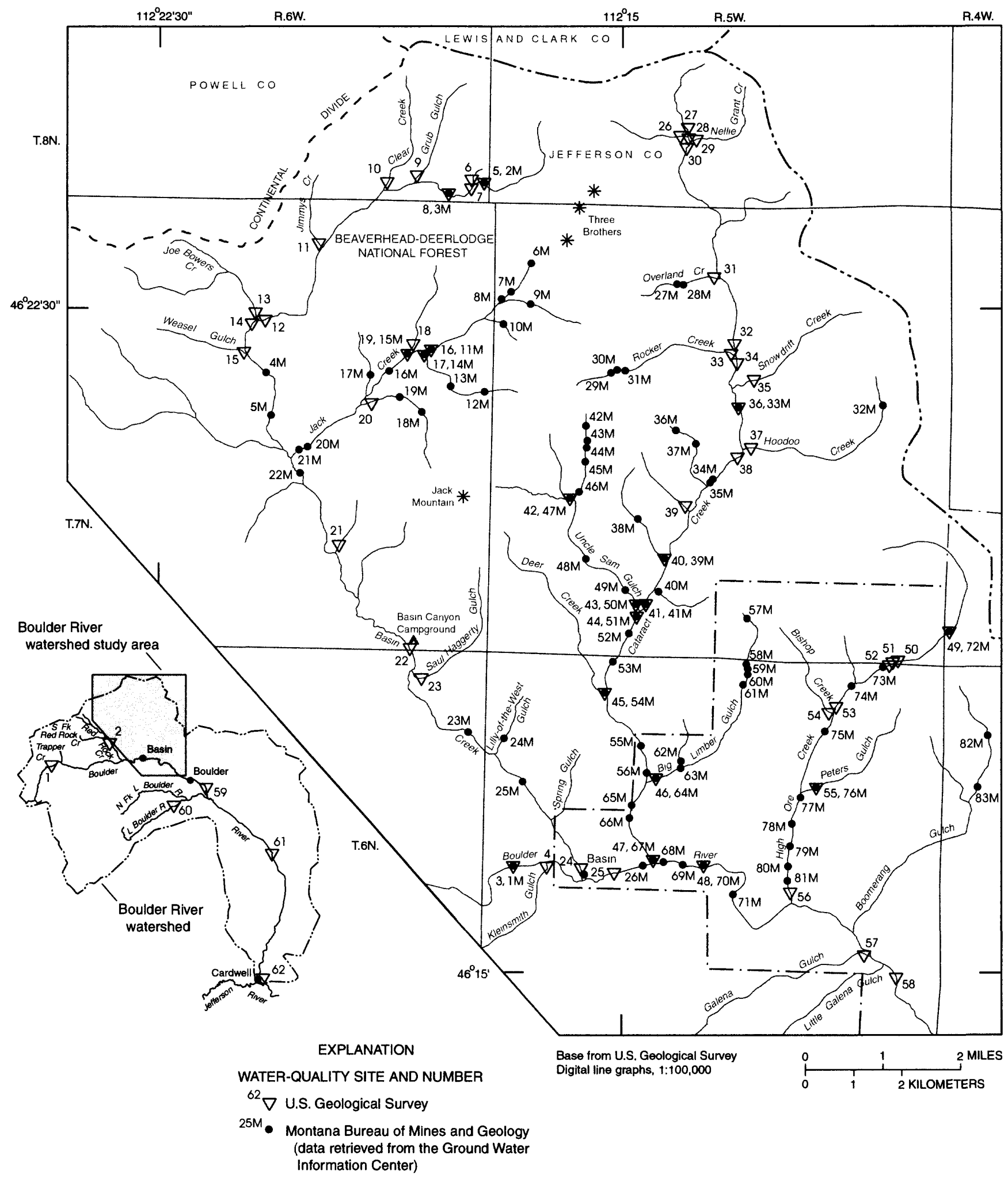

Figure 2. Water-quality sampling sites in the Boulder River watershed, Montana. 
streams throughout the northern Rocky Mountains. Streamflow is dominated by snowmelt runoff, which typically occurs sometime between April and June, and is augmented in some years by rain. Streamflow typically peaks in May or June and then decreases as the shallow ground-water system drains. Low streamflow conditions are typical from July to March.

\section{Acknowledgments}

The authors acknowledge with appreciation the many individuals who assisted in this study. Particular thanks for assistance are given to Pat Barringer, Robert Wintergerst, and Ray TeSoro with the USDA-Forest Service and Tim M. Bozorth with the Bureau of Land Management. Assisting in the collection of waterquality data were Fred A. Bailey, Michael R. Cannon, Kent A. Dodge, DeAnn M. Dutton, Philip L. Karper, Briant A. Kimball, and Kevin L. Sattler with the USGS. Appreciation is extended to the landowners in the study area for allowing access to their property.

\section{WATER-QUALITY DATA}

Water-quality samples were collected for this study by the USGS at 62 sites (table 1) from October 1996 through September 1999. Water-quality data for field parameters, major ions, nutrients, and trace elements are presented in table 2 . Water-sampling sites were located throughout the watershed and at locations upstream and downstream from inactive mines (fig. 2). Reference sites located in drainages that had a geologic setting similar to the study area, except without the mineralization, also were sampled to provide a reference for water-quality comparison with mineralized areas. These reference sites (fig. 2) included the Boulder River above Trapper Creek (site 1), Red Rock Creek below South Fork (site 2), and the Little Boulder River above North Fork (site 60). Samples were collected at three sites on the Boulder River (sites 59, 61, and 62) downstream from the study area to determine the extent of trace-element enrichment from the mining district.

Historical data for field parameters and selected dissolved metals for 83 sites (table 3) were compiled and are presented in table 4. Most of these data were collected during inventories of inactive mines in the Boulder River watershed that were conducted by the MBMG (Marvin and others, 1997; Metesh and others,
1994, 1995, 1996). Data were retrieved from the Ground Water Information Center database at the MBMG in Butte, Mont.

\section{Sampling and Processing Methods}

Water samples typically were collected from multiple verticals across the stream using depth- and width-integration methods described by Knapton (1985) and Edwards and Glysson (1988). These methods provide a vertically and laterally dischargeweighted sample that is representative of the entire flow through the cross section of a stream. Grab samples were collected when streamflow was too low to allow use of a depth-integrating sampler. Sampling equipment consisted of standard USGS depth-integrating suspended-sediment samplers (DH-81, DH-48, and D-74TM), which are either constructed of plastic or coated with a non-metallic epoxy paint, and equipped with interchangeable nylon nozzles for sampling a wide range of stream depths and velocities.

Field measurements of specific conductance, $\mathrm{pH}$, and water temperature were made during collection of periodic water-quality samples. Samples were processed according to procedures described by Horowitz and others (1994), Ward and Harr (1990), and Knapton (1985). Samples for some constituents were filtered using filters having a pore size of $0.45 \mu \mathrm{m}$; analytical data for these samples are considered dissolved. Instantaneous streamflow at the time of sample collection was determined either by direct measurement, estimation, or stage-discharge rating (Rantz and others, 1982).

\section{Laboratory Analysis}

Water samples were analyzed by the USGS National Water Quality Laboratory (NWQL) in Arvada and Lakewood, Colo. Initially, samples were analyzed for an extensive suite of trace elements. After reviewing analytical data for high-flow and low-flow samples from many sites throughout the study area, analyses were discontinued for selected trace elements that consistently had concentrations less than the minimum reporting level. Selected samples collected for assessment of aquatic-habitat conditions were analyzed for nutrients. Analytical methods are described by Faires (1993), Fishman and Friedman (1989), Fishman 


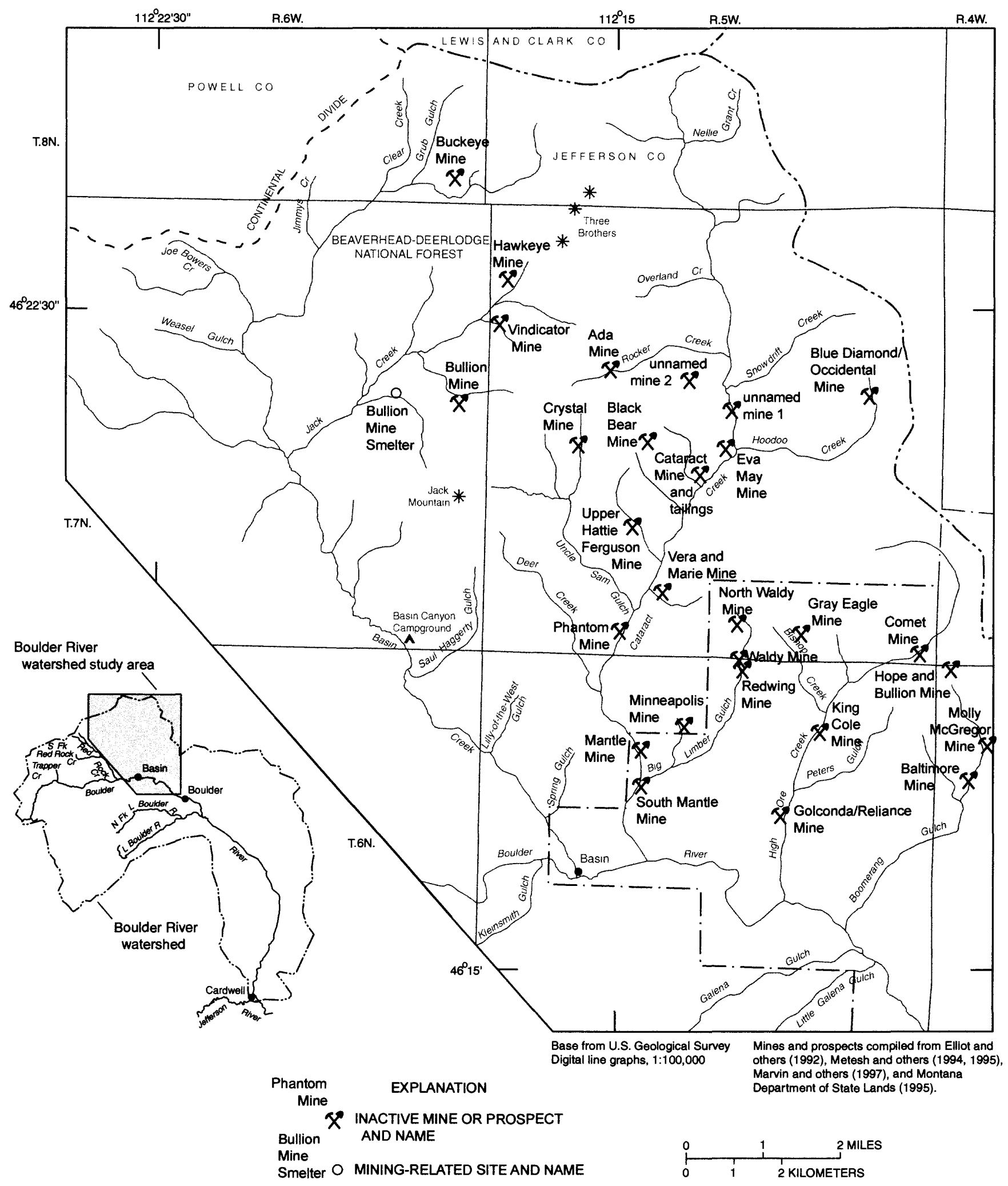

Figure 3. Selected inactive mines, prospects, and mining-related sites in the Boulder River watershed, Montana. 
(1993), Garbarino (1999), Garbarino and Struzeski (1998), Hoffman and others (1996), Jones and Garbarino (1999), and Patton and Truitt (1992).

Cross-sectional water samples also were collected for analysis of suspended sediment for most of the water-quality samples (table 2). These samples were analyzed for suspended-sediment concentration and the percentage of suspended sediment finer than 0.062-mm diameter (silt size and smaller) by the USGS Montana District sediment laboratory in Helena, Mont., according to methods described by Guy (1969) and Lambing and Dodge (1993).

\section{Quality-Assurance Data}

Data-collection and analytical procedures used in this study incorporated practices designed to control, verify, and assess the quality of sample data. Qualityassurance procedures used for the collection and field processing of water-quality samples are described by Horowitz and others (1994), Ward and Harr (1990), Edwards and Glysson (1988), Knapton and Nimick (1991), and Knapton (1985). Standard procedures used by the NWQL for internal sample handling and quality assurance are described by Friedman and Erdmann (1982), Jones (1987), and Pritt and Raese (1995). Quality-assurance procedures used by the Montana District sediment laboratory are described by Lambing and Dodge (1993).

The quality of analytical results reported for water-quality samples can be evaluated with data from quality-control samples that were submitted from the field and analyzed concurrently in the laboratory with routine samples. These quality-control samples consisted of duplicates and blanks, which provide quantitative information on the precision and bias of the overall field and laboratory process. The total number of quality-control samples represented about 6 percent of the total number of water-quality samples.

In addition to quality-control samples submitted from the field, internal quality-assurance practices at the NWQL are performed systematically to provide quality control of analytical procedures (Pritt and Raese, 1995). These internal practices include analyses of quality-control samples such as calibration standards, standard reference water samples, replicate samples, deionized-water blanks, or spiked samples at a proportion equivalent to at least 10 percent of the sample load.

Duplicate samples are two samples considered to be essentially identical in composition. Precision of analytical results for field duplicates is affected by many sources of variability within the field and laboratory environments, including sample collection, processing, and analysis. For this study, duplicate samples were obtained in the field to provide data on precision (reproducibility) of analytical results for samples exposed to all sources of variability. Duplicate samples were obtained in the field by splitting a single sample into two subsamples, which then were analyzed separately. Analyses of field duplicates are presented in table 5.

Precision of analytical results for any constituent can be determined from the relative percent difference (RPD) of the concentrations of the constituent in duplicate analyses. RPD for each duplicate sample pair is computed from the equation

$$
\mathrm{RPD}=\frac{\text { difference between duplicate values }}{\text { mean of duplicate values }} \times 100
$$

Analytical data in table 5 generally indicate good precision, with RPD values for most constituents of 10 percent or less. However, the precision of total recoverable analyses for trace elements is less; the RPDs for some analyses are as high as 29 percent for aluminum, arsenic, and copper and almost 50 percent for iron and lead. Although the precision was poor for several samples, the infrequent and random occurrence of affected constituents indicates no systematic analytical problem.

Blank samples of deionized water were routinely analyzed to identify the presence and magnitude of contamination that potentially could bias analytical results. The particular type of blank sample routinely tested was a "field" blank. A field blank is an aliquot of deionized water, which is certified as trace-element free and is processed through the sampling equipment used to collect stream samples. These blanks are then subjected to the same processing (filtration, preservation, transportation, and laboratory handling) as stream samples. Blank samples were analyzed for the same constituents as those of stream samples to identify whether any detectable concentrations exist. Analytical results for field blanks are presented in table 6 .

A field blank with constituent concentrations equal to or less than the minimum reporting level for 
the analytical method indicates that the entire process of sample collection, field processing, and laboratory analysis is presumably free of significant contamination. If detectable concentrations in field blanks were equal to or greater than twice the minimum reporting level (typical measurement precision at the detection level), the concentrations were noted during data review. Sporadic, infrequent exceedances of twice the minimum reporting level probably represent random contamination or instrument calibration error that is not persistent in the process and which is not likely to cause significant positive bias in a long-term record of analytical results. With the exception noted in the next paragraph, constituent concentrations in field blanks were almost always less than the minimum reporting level. Therefore, the analytical results for field blanks indicate no systematic contamination that would bias the reported water-quality data for stream samples.

At the beginning of this study, all samples were preserved in the field with concentrated nitric acid dispensed from a pre-filled and sealed glass vial. Blank samples collected during this period consistently had dissolved aluminum concentrations of 3 to $4 \mu \mathrm{g} / \mathrm{L}$. This apparent contamination was attributed to the glass vials. Subsequently, all samples were preserved with concentrated nitric acid either dispensed in the field from pre-filled polytetrafluorethylene vials or in the laboratory within 24 hours of sample collection from the manufacturer's acid container using a pipettor. Blank samples preserved using either of these two latter methods showed no consistent pattern of contamination. On the basis of these quality-assurance results, the dissolved aluminum concentration initially reported by NWQL for each stream sample preserved with acid dispensed from a glass vial was decreased by $3.5 \mu \mathrm{g} / \mathrm{L}$, which was the average dissolved aluminum concentration in the five blank samples preserved with acid dispensed from a glass vial. These adjusted values are reported in tables 2 and 5.

\section{REFERENCES CITED}

Buxton, H.T., Nimick, D.A., von Guerard, Paul, Church, S.E., Frazier, Ann, Gray, J.R., Lipin, B.R., Marsh, S.P., Woodward, Daniel, Kimball, Briant, Finger, Susan, Ischinger, Lee, Fordham, J.C., Power, M.S., Bunck, Christine, and Jones, J.W., 1997, A science-based, watershed strategy to support effective remediation of abandoned mine lands: Proceedings of the Fourth International Conference on Acid Rock Drainage, Vancouver, B.C., May 31-June 6, 1997, p. 1869-1880.
Edwards, T.K., and Glysson, G.D., eds., 1988, Field methods for measurement of fluvial sediment: U.S. Geological Survey Open-File Report 86-531, 118 p.

Elliot, J.E., Loen, J.S., Wise, K.K., and Blaskowski, M.J., 1992, Maps showing locations of mines and prospects in the Butte 1 degree $x 2$ degree quadrangle, western Montana: U.S. Geological Survey Miscellaneous Investigations Series Map I-2050-C, 2 plates, scale $1: 250,000$.

Faires, L.M., 1993, Methods of analysis by the U.S. Geological Survey National Water Quality Laboratory--Determination of metals in water by inductively coupled plasma-mass spectrometry: U.S. Geological Survey Open-File Report 92-634, 28 p.

Farag, A.M., Woodward, D.F., Skaar, D., and Brumbaugh, W.G., 1999, Characterizing the aquatic health in the Boulder River watershed, Montana, in Morganwalp, D.W., and Buxton, H.T., eds., U.S. Geological Survey Toxic Substances Hydrology Program--Proceedings of the Technical Meeting, Charleston, South Carolina, March 8-12, 1999--Volume 1 of 3--Contamination from Hardrock Mining: U.S. Geological Survey WaterResources Investigations Report 99-4018A, p. 55-58.

Fishman, M.J., 1993, Methods of analysis by the U.S. Geological Survey National Water Quality Laboratory--Determination of inorganic and organic constituents in water and fluvial sediments: U.S. Geological Survey Open-File Report 93-125, 217 p.

Fishman, M.J., and Friedman, L.C., 1989, Methods for determination of inorganic substances in water and fluvial sediments: U.S. Geological Survey Techniques of Water-Resources Investigations, book 5, chap. A1, $709 \mathrm{p}$.

Friedman, L.C., and Erdman, D.E., 1982, Quality assurance practices for the chemical and biological analyses of water and fluvial sediments: U.S. Geological Survey Techniques of Water-Resources Investigations, book 5 , chap. A6, $181 \mathrm{p}$.

Garbarino, J.R., 1999, Methods of analysis by the U.S. Geological Survey National Water Quality Laboratory--Determination of dissolved arsenic, boron, lithium, selenium, strontium, thallium, and vanadium using inductively coupled plasma-mass spectrometry: U.S. Geological Survey Open-File Report 99-093, 31 p.

Garbarino, J.R., and Struzeski, T.M., 1998, Methods of analysis by the U.S. Geological Survey National Water Quality Laboratory--Determination of elements in whole-water digests using inductively coupled plasmaoptical emission spectrometry and inductively coupled plasma-mass spectrometry: U.S. Geological Survey Open-File Report 98-165, 101 p. 
Guy, H.P., 1969, Laboratory theory and methods for sediment analysis: U.S. Geological Survey Techniques of Water-Resources Investigations, book 5 , chap. $\mathrm{Cl}$, $58 \mathrm{p}$.

Hoffman, G.L., Fishman, M.J., and Garbarino, J.R., 1996, Methods of analysis by the U.S. Geological Survey National Water Quality Laboratory--In-bottle acid digestion of whole-water samples: U.S. Geological Survey Open-File Report 96-225, 28 p.

Horowitz, A.J., Demas, C.R., Fitzgerald, K.K., Miller, T.L., and Rickert, D.A., 1994, U.S. Geological Survey protocol for the collection and processing of surfacewater samples for the subsequent determination of inorganic constituents in filtered water: U.S. Geological Survey Open-File Report 94-539, 57 p.

Jones, B.E., 1987, Quality control manual of the U.S. Geological Survey's National Water Quality Laboratory: U.S. Geological Survey Open-File Report $87-457,17 \mathrm{p}$.

Jones, S.R., and Garbarino, J.R., 1999, Methods of analysis by the U.S. Geological Survey National Water Quality Laboratory--Determination of arsenic and selenium in water and sediment by graphite furnace-atomic absorption spectrometry: U.S. Geological Survey Open-File Report 98-639, 39 p.

Knapton, J.R., 1985, Field guidelines for collection, treatment, and analysis of water samples, Montana District: U.S. Geological Survey Open-File Report 85409, 86 p.

Knapton, J.R., and Nimick, D.A., 1991, Quality assurance for water-quality activities of the U.S. Geological Survey in Montana: U.S. Geological Survey Open-File Report 91-216, $41 \mathrm{p}$.

Lambing, J.H., and Dodge, K.A., 1993, Quality assurance for laboratory analysis of suspended-sediment samples by the U.S. Geological Survey in Montana: U.S. Geological Survey Open-File Report 93-131, 34 p.

Marvin, R.K., Metesh, J.J., Hargrave, P.A., Lonn, J.D., Watson, J.E., Bowler, T.P., and Madison, J.P., 1997, Abandoned-inactive mines of Montana, U.S. Bureau of Land Management: Montana Bureau of Mines and Geology Open-File Report 348, 513 p.

Metesh, J.J., Lonn, J.D., Duaime, T.E., and Wintergerst, Robert, 1994, Abandoned-inactive mines in the Deerlodge National Forest, Volume I--Basin Creek drainage: Montana Bureau of Mines and Geology Open-File Report 321, 123 p.
Metesh, J.J., Lonn, J.D., Duaime, T.E., and Marvin, R.K., Wintergerst, Robert, 1995, Abandoned-inactive mines in the Deerlodge National Forest, Volume II--Cataract Creek drainage: Montana Bureau of Mines and Geology Open-File Report 344, 163 p.

Metesh, J.J., Lonn, J.D., Marvin, R.K, Madison, J.P., and Wintergerst, Robert, 1996, Abandoned-inactive mines in the Deerlodge National Forest, Volume V--Jefferson River drainage: Montana Bureau Of Mines and Geology Open-File Report 347, 157 p.

Montana Department of State Lands, 1995, Abandoned hardrock mine priority sites, 1995 summary report: Prepared by Pioneer Technical Services, Inc., Butte, Mont., for the Abandoned Mine Reclamation Bureau, variously paged.

Nimick, D.A., Church, S.E., Cleasby, T.E., Fey, D.L., Kimball, B.A., Leib, K.J., Mast, A.M., and Wright, W.G., 1999, Characterization of metals in water and bed sediment in two watersheds affected by historical mining in Montana and Colorado, in Morganwalp, D.W., and Buxton, H.T., eds., U.S. Geological Survey Toxic Substances Hydrology Program--Proceedings of the Technical Meeting, Charleston, South Carolina, March 8-12, 1999--Volume 1 of 3--Contamination from Hardrock Mining: U.S. Geological Survey WaterResources Investigations Report 99-4018A, p. 11-18.

Patton, C.J., and Truitt, E.P., 1992, Methods of analysis by the U.S. Geological Survey National Water Quality Laboratory--Determination of total phosphorus by a Kjeldahl digestion method and an automated colorimetric finish that includes dialysis: U.S. Geological Survey Open-File Report 92-146, 39 p.

Pritt, J.W., and Raese, J.W., eds., 1995, Quality assurance/ quality control manual--National Water Quality Laboratory: U.S. Geological Survey Open-File Report 95-443, $35 \mathrm{p}$.

Rantz, S.E., and others, 1982, Computation of discharge: U.S. Geological Survey Water-Supply Paper 2175, 2 v., $631 \mathrm{p}$.

Ward, J.R., and Harr, C.A., eds., 1990, Methods for collection and processing of surface-water and bedmaterial samples for physical and chemical analyses: U.S. Geological Survey Open-File Report 90-140, 71 p. 
DATA

DATA 9 
Table 1. Water-quality sampling sites in the Boulder River watershed, Montana, 1996-99

\begin{tabular}{|c|c|c|}
\hline $\begin{array}{c}\text { Site } \\
\text { number } \\
\text { (fig. 2) }\end{array}$ & $\begin{array}{c}\text { Station } \\
\text { identification number }^{1}\end{array}$ & Station name \\
\hline \multicolumn{3}{|r|}{ BOULDER RIVER } \\
\hline 1 & 461541112291001 & Boulder River above Trapper Creek, near Basin \\
\hline 2 & 461753112205001 & Red Rock Creek below South Fork, near Basin \\
\hline 3 & 06031450 & Boulder River above Kleinsmith Gulch, near Basin \\
\hline 4 & 461612112160401 & Kleinsmith Gulch at Basin \\
\hline 25 & 461609112150401 & Boulder River below Basin Creek, near Basin \\
\hline 48 & 06032000 & Boulder River near Basin \\
\hline 57 & 461517112110401 & Galena Gulch at mouth, near Boulder \\
\hline 58 & 06032400 & Boulder River below Little Galena Gulch, near Boulder \\
\hline 59 & 06033000 & Boulder River near Boulder \\
\hline 60 & 461135112085101 & Little Boulder River above North Fork, near Boulder \\
\hline 61 & 460649111550701 & Boulder River at County Bridge, near Boulder \\
\hline 62 & 06033900 & Boulder River near Cardwell \\
\hline \multicolumn{3}{|r|}{ BASIN CREEK } \\
\hline 5 & 462348112172901 & Basin Creek above Buckeye Mine, near Basin \\
\hline 6 & 462349112173101 & Buckeye Mine tributary near Basin \\
\hline 7 & 462345112173601 & Basin Creek below Buckeye Mine tributary, near Basin \\
\hline 8 & 462340112175701 & Basin Creek below Buckeye Mine, near Basin \\
\hline 9 & 462350112182701 & Grub Gulch near Basin \\
\hline 10 & 462341112190401 & Clear Creek near Basin \\
\hline 11 & 462305112200001 & Jimmys Creek near Basin \\
\hline 12 & 462218112203901 & Basin Creek above Joe Bowers Creek, near Basin \\
\hline 13 & 462216112210101 & Joe Bowers Creek near Basin \\
\hline 14 & 462212112210201 & Basin Creek at Joe Bowers Creek, near Basin \\
\hline 15 & 462151112211001 & Weasel Gulch near Basin \\
\hline 16 & 462155112181501 & Jack Creek above Bullion Mine tributary, near Basin \\
\hline 17 & 462153112181701 & Bullion Mine tributary at mouth, near Basin \\
\hline 18 & 462158112182501 & Jack Creek tributary near Basin \\
\hline 19 & 462152112183301 & Jack Creek below Bullion Mine tributary, near Basin \\
\hline 20 & 462122112185901 & Bullion Mine Smelter tributary near Basin \\
\hline 21 & 461944112193501 & Basin Creek tributary near Basin \\
\hline 22 & 461834112182301 & Basin Creek at Basin Canyon Campground, near Basin \\
\hline 23 & 461817112182001 & Saul Haggerty Gulch at mouth, near Basin \\
\hline 24 & 06031600 & Basin Creek at Basin \\
\hline \multicolumn{3}{|r|}{ CATARACT CREEK } \\
\hline 26 & 462422112140601 & Cataract Creek above Nellie Grant Creek, near Basin \\
\hline 27 & 462424112140401 & Cataract Creek tributary above Nellie Grant Creek, near Basin \\
\hline 28 & 462420112140301 & Cataract Creek 100 feet above Nellie Grant Creek, near Basin \\
\hline 29 & 462419112135801 & Nellie Grant Creek near Basin \\
\hline 30 & 462415112140201 & Cataract Creek below Nellie Grant Creek, near Basin \\
\hline 31 & 462250112133201 & Overland Creek near Basin \\
\hline 32 & 462200112131301 & Cataract Creek above Rocker Creek, near Basin \\
\hline 33 & 462157112131601 & Rocker Creek at mouth, near Basin \\
\hline 34 & 462154112131301 & Cataract Creek below Rocker Creek, near Basin \\
\hline 35 & 462137112125601 & Snowdrift Creek near Basin \\
\hline 36 & 462119112130901 & Cataract Creek above Hoodoo Creek, near Basin \\
\hline 37 & 462053112131401 & Hoodoo Creek near Basin \\
\hline 38 & 462045112131901 & Cataract Creek below Hoodoo Creek, near Basin \\
\hline
\end{tabular}


Table 1. Water-quality sampling sites in the Boulder River watershed, Montana, 1996-99 (Continued)

\begin{tabular}{cll}
$\begin{array}{c}\text { Site } \\
\text { number } \\
\text { (fig. 2) }\end{array}$ & \multicolumn{1}{c}{$\begin{array}{c}\text { Station } \\
\text { identification number }\end{array}$} & \\
\hline 39 & 462013112140001 & \multicolumn{1}{c}{ CATARACT CREEK--Continued } \\
Cataract Creek tributary 1.4 mi above Uncle Sam Gulch, near Basin \\
40 & 461937112142001 & Cataract Creek tributary 0.6 mi above Uncle Sam Gulch, near Basin \\
41 & 461905112144201 & Cataract Creek above Uncle Sam Gulch, near Basin \\
42. & 462056112161201 & Unnamed tributary to Uncle Sam Gulch, near Basin \\
43 & 461904112144401 & Uncle Sam Gulch at mouth, near Basin \\
44 & 461902112144501 & Cataract Creek below Uncle Sam Gulch, near Basin \\
45 & 461807112151901 & Deer Creek near Basin \\
46 & 461708112143201 & Big Limber Gulch near Basin \\
47 & 06031960 & Cataract Creek at Basin \\
& & HIGH ORE CREEK \\
49 & 461852112094201 & High Ore Creek above Comet Mine, near Basin \\
50 & 461831112103701 & High Ore Creek above Comet Mine outflow, near Basin \\
51 & 461830112103901 & Comet Mine outflow near Basin \\
52 & 461829112104301 & High Ore Creek below Comet Mine outflow, near Basin \\
53 & 461757112113501 & High Ore Creek above Bishop Creek, near Basin \\
54 & 461755112113701 & Bishop Creek near Basin \\
55 & 461703112120201 & Peters Gulch near Basin \\
56 & 06032300 & High Ore Creek near Basin \\
\hline
\end{tabular}

${ }^{1}$ Fifteen-digit station-identification number is a unique identifier that represents the approximate latitude and longitude location of the site (first 13 digits), plus a sequence number (last two digits). Eight-digit station identification number represents the standard USGS streamflow-gaging station numbering system wherein numbers increase in a downstream direction according to geographic location within the drainage basin. 
Table 2. Water-quality data for streams in the Boulder River watershed, Montana, 1996-99

[Samples collected by the U.S. Geological Survey. ANC, acid-neutralizing capacity determined on unfiltered samples, formerly referred to as alkalinity. Abbreviations: $\mathrm{ft}^{3} / \mathrm{s}$, cubic feet per second; ${ }^{\circ} \mathrm{C}$, degrees Celsius; e, estimated; lab, laboratory; $\mu \mathrm{g} / \mathrm{L}$, micrograms per liter; $\mu \mathrm{S} / \mathrm{cm}$, microsiemens per centimeter at $25^{\circ} \mathrm{C}$; mi, mile; $\mathrm{mg} / \mathrm{L}$, milligrams per liter; $\mathrm{mm}$, millimeters. Symbols: <, less than minimum reporting level; --, no data]

\begin{tabular}{|c|c|c|c|c|c|c|c|c|c|c|}
\hline $\begin{array}{c}\text { Site } \\
\text { num- } \\
\text { ber } \\
\text { (fig. 2) }\end{array}$ & Station name & Date & Time & $\begin{array}{c}\text { Dis- } \\
\text { charge, } \\
\text { instanta- } \\
\text { neous } \\
\left(\mathrm{ft}^{3} / \mathrm{s}\right)\end{array}$ & $\begin{array}{l}\text { Sedi- } \\
\text { ment, } \\
\text { sus- } \\
\text { pended } \\
\text { (mg/L) }\end{array}$ & $\begin{array}{c}\text { Sedi- } \\
\text { ment, } \\
\text { sus- } \\
\text { pended, } \\
\text { diameter } \\
\text { (percent } \\
\text { finer } \\
\text { than } \\
0.062 \\
\text { mm) }\end{array}$ & $\begin{array}{c}\text { Spe- } \\
\text { cific } \\
\text { con- } \\
\text { duct- } \\
\text { ance, } \\
\text { field } \\
(\mu \mathrm{S} / \mathrm{cm})\end{array}$ & $\begin{array}{c}\text { pH, } \\
\text { field } \\
\text { (stan- } \\
\text { dard } \\
\text { units) }\end{array}$ & $\begin{array}{l}\text { Tem- } \\
\text { pera- } \\
\text { ture, } \\
\text { water } \\
\left({ }^{\circ} \mathrm{C}\right)\end{array}$ & $\begin{array}{c}\text { Hard- } \\
\text { ness } \\
\text { (mg/L } \\
\text { as } \\
\left.\mathrm{CaCO}_{3}\right)\end{array}$ \\
\hline \multicolumn{11}{|c|}{ BOULDER RIVER } \\
\hline \multirow[t]{4}{*}{1} & Boulder River above Trapper Creek, near Basin & $07-23-97$ & 1000 & 15 & 5 & 87 & 100 & 8.1 & 12.5 & 38 \\
\hline & & $09-22-97$ & 0940 & 3.8 & 2 & 95 & 132 & 8.0 & 6.0 & 53 \\
\hline & & $02-12-98$ & 1310 & 2.3 & 2 & 89 & 134 & 8.0 & 0.0 & 52 \\
\hline & & $05-06-98$ & 0945 & 83 & 24 & 33 & 55 & 7.6 & 4.0 & 21 \\
\hline 2 & Red Rock Creek below South Fork, near Basin & $09-25-98$ & 0910 & 1.3 & -- & -- & 130 & 8.2 & 7.0 & 56 \\
\hline \multirow[t]{18}{*}{3} & Boulder River above Kleinsmith Gulch, near Basin & $10-18-96$ & 1255 & 19 & 3 & 79 & 143 & 7.4 & 3.0 & 56 \\
\hline & & $11-01-96$ & 0930 & 13 & 3 & 60 & 155 & 8.1 & 0.0 & 57 \\
\hline & & $12-03-96$ & 1000 & 12 & 2 & 94 & 145 & 7.6 & 0.0 & 54 \\
\hline & & $02-11-97$ & 1000 & 17 & 4 & 76 & 163 & 7.7 & 0.0 & 61 \\
\hline & & $04-18-97$ & 1000 & 106 & 21 & 85 & 132 & 8.0 & 3.5 & 50 \\
\hline & & $05-07-97$ & 1005 & 276 & 50 & 36 & 101 & 7.9 & 4.5 & 37 \\
\hline & & $05-14-97$ & 1030 & 552 & 57 & 43 & 80 & 7.7 & 9.5 & 28 \\
\hline & & $05-23-97$ & 1000 & 603 & 22 & 44 & 68 & 7.6 & 7.5 & 22 \\
\hline & & $06-02-97$ & 0915 & 725 & 52 & 43 & 65 & 7.6 & 9.0 & 24 \\
\hline & & $06-18-97$ & 0945 & 455 & 38 & 36 & 89 & 7.8 & 12.5 & 34 \\
\hline & & $07-17-97$ & 0915 & 111 & 15 & 87 & 124 & 7.9 & 15.0 & 49 \\
\hline & & $09-23-97$ & 0845 & 25 & 3 & 91 & 154 & 8.2 & 6.0 & 55 \\
\hline & & $02-10-98$ & 1500 & 21 & 6 & 86 & 150 & 7.7 & 0.0 & 58 \\
\hline & & $04-29-98$ & 0900 & 168 & 28 & 57 & 104 & 8.2 & 4.0 & 41 \\
\hline & & $05-05-98$ & 0845 & 300 & 29 & 59 & 74 & 8.0 & 4.5 & 28 \\
\hline & & $09-23-98$ & 1415 & 23 & 3 & 69 & 144 & - & 11.5 & 57 \\
\hline & & $05-25-99$ & 1000 & 318 & 76 & 54 & 63 & 8.0 & 8.0 & 22 \\
\hline & & $09-14-99$ & 0930 & 8.6 & 2 & 86 & 152 & 8.3 & 6.5 & 56 \\
\hline \multirow[t]{2}{*}{4} & Kleinsmith Gulch at Basin & $10-30-96$ & 1515 & .15 & -- & - & 236 & -- & 0.0 & - \\
\hline & & $05-13-97$ & 1400 & .43 & -- & -- & 174 & -- & 10.0 & -- \\
\hline \multirow[t]{4}{*}{25} & Boulder River below Basin Creek, near Basin & $07-24-97$ & 0930 & 94 & 6 & 86 & 125 & 7.9 & 13.5 & 47 \\
\hline & & $09-22-97$ & 1200 & 28 & 3 & 79 & 147 & 8.2 & 12.0 & 59 \\
\hline & & $02-11-98$ & 1430 & 25 & 3 & 82 & 146 & 7.9 & 0.0 & 55 \\
\hline & & $05-05-98$ & 1130 & 436 & 29 & 54 & 65 & 7.8 & -- & 24 \\
\hline \multirow[t]{4}{*}{48} & Boulder River near Basin & $07-24-97$ & 1140 & 106 & 5 & 80 & 122 & 8.0 & 15.5 & 47 \\
\hline & & $09-22-97$ & 1400 & 33 & 3 & 15 & 148 & 8.0 & 13.0 & 60 \\
\hline & & $02-09-98$ & 1130 & 26 & 2 & 83 & 146 & 7.7 & 0.0 & 57 \\
\hline & & $05-05-98$ & 1415 & 513 & 24 & 64 & 63 & 7.7 & -- & 24 \\
\hline \multirow[t]{2}{*}{57} & Galena Gulch at mouth, near Boulder & $10-31-96$ & 1230 & .40 & 1 & 89 & 281 & 8.3 & 0.0 & 120 \\
\hline & & $05-13-97$ & 1215 & 5.4 & 75 & 82 & 113 & 7.8 & 10.0 & 43 \\
\hline
\end{tabular}


Table 2. Water-quality data for streams in the Boulder River watershed, Montana, 1996-99 (Continued)

\begin{tabular}{|c|c|c|c|c|c|c|c|c|c|c|}
\hline $\begin{array}{c}\text { Site } \\
\text { num- } \\
\text { ber } \\
\text { (fig. 2) }\end{array}$ & Date & $\begin{array}{c}\text { Cal- } \\
\text { cium, } \\
\text { dissolved } \\
\text { (mg/L } \\
\text { as Ca) }\end{array}$ & $\begin{array}{l}\text { Magne- } \\
\text { sium, } \\
\text { dissolved } \\
\text { (mg/L } \\
\text { as } \mathbf{M g} \text { ) }\end{array}$ & $\begin{array}{c}\text { Sodium, } \\
\text { dissolved } \\
\text { (mg/L } \\
\text { as Na) }\end{array}$ & $\begin{array}{l}\text { Potas- } \\
\text { sium, } \\
\text { dissolved } \\
\text { (mg/L } \\
\text { as K) }\end{array}$ & $\begin{array}{c}\mathrm{ANC}, \\
\text { lab } \\
(\mathrm{mg} / \mathrm{L} \\
\left.\text { as } \mathrm{CaCO}_{3}\right)\end{array}$ & $\begin{array}{c}\text { Sulfate, } \\
\text { dissolved } \\
(\mathrm{mg} / \mathrm{L} \\
\left.\text { as } \mathrm{SO}_{4}\right)\end{array}$ & $\begin{array}{c}\text { Chloride, } \\
\text { dissolved } \\
\text { (mg/L } \\
\text { as } \mathrm{Cl} \text { ) }\end{array}$ & $\begin{array}{c}\text { Fluoride, } \\
\text { dissolved } \\
\text { (mg/L } \\
\text { as F) }\end{array}$ & $\begin{array}{c}\text { Silica, } \\
\text { dissolved } \\
(\mathrm{mg} / \mathrm{L} \\
\left.\text { as } \mathrm{SiO}_{2}\right)\end{array}$ \\
\hline
\end{tabular}

\begin{tabular}{|c|c|c|c|c|c|c|c|c|c|c|c|}
\hline \multirow[b]{2}{*}{1} & \multicolumn{11}{|c|}{ BOULDER RIVER } \\
\hline & $07-23-97$ & 12 & 2.0 & 4.5 & 1.3 & 45 & 5.5 & 0.41 & $<0.10$ & 21 & 74 \\
\hline & $09-22-97$ & 16 & 3.1 & 6.7 & 1.5 & 60 & 8.3 & .85 & $<.10$ & 22 & 95 \\
\hline & $02-12-98$ & 16 & 3.1 & 6.6 & 1.5 & 58 & 11 & .76 & $<.10$ & 23 & 97 \\
\hline & $05-06-98$ & 6.7 & 1.1 & 2.7 & .96 & 22 & 4.2 & .41 & $<.10$ & 15 & 44 \\
\hline 2 & $09-25-98$ & 16 & 3.7 & 3.6 & 1.1 & 60 & 6.7 & .47 & $<.10$ & 17 & 85 \\
\hline \multirow[t]{18}{*}{3} & $10-18-96$ & 17 & 3.4 & 8.2 & 1.4 & 59 & 14 & 2.3 & .10 & 20 & 102 \\
\hline & $11-01-96$ & 17 & 3.6 & 8.8 & 1.4 & 60 & 17 & 2.2 & .10 & 22 & 108 \\
\hline & $12-03-96$ & 16 & 3.3 & 8.1 & 1.4 & 56 & 14 & 1.6 & .10 & 21 & 100 \\
\hline & $02-11-97$ & 18 & 3.8 & -- & -- & -- & -- & -- & -- & -- & -- \\
\hline & $04-18-97$ & 15 & 3.2 & 6.1 & 1.9 & 50 & 13 & 2.2 & $<.10$ & 17 & 89 \\
\hline & $05-07-97$ & 11 & 2.4 & 5.0 & 1.4 & 35 & 11 & 1.3 & .10 & 18 & 72 \\
\hline & $05-14-97$ & 8.3 & 1.7 & 4.2 & 1.1 & 28 & 8.2 & .79 & $<.10$ & 18 & 59 \\
\hline & $05-23-97$ & 6.7 & 1.3 & 3.5 & .97 & 23 & 5.6 & .64 & $<.10$ & 18 & 51 \\
\hline & $06-02-97$ & 7.0 & 1.5 & 3.5 & .99 & 25 & 5.8 & .60 & $<.10$ & 16 & 51 \\
\hline & $06-18-97$ & 10 & 2.1 & 4.4 & 1.1 & 36 & 5.2 & .80 & $<.10$ & 20 & 66 \\
\hline & $07-17-97$ & 15 & 2.8 & 6.5 & 1.4 & 50 & 9.1 & 1.6 & $<.10$ & 22 & 89 \\
\hline & $09-23-97$ & 17 & 3.2 & 7.7 & 1.6 & 63 & 13 & 2.2 & .12 & 21 & 103 \\
\hline & $02-10-98$ & 17 & 3.7 & 8.0 & 1.3 & 57 & 15 & 2.6 & $<.10$ & 22 & 104 \\
\hline & $04-29-98$ & 12 & 2.5 & 4.9 & 1.5 & 42 & 8.7 & 1.4 & $<.10$ & 16 & 72 \\
\hline & $05-05-98$ & 8.6 & 1.7 & 3.9 & 1.1 & 30 & 5.9 & .75 & .10 & 15 & 55 \\
\hline & $09-23-98$ & 17 & 3.4 & 8.6 & 1.3 & 64 & 11 & 2.1 & .13 & 21 & 104 \\
\hline & $05-25-99$ & 6.6 & 1.4 & 3.4 & 1.0 & 25 & 4.9 & .66 & -- & -- & - \\
\hline & $09-14-99$ & 17 & 3.4 & 9.8 & 1.8 & 62 & 12 & 2.4 & - & -- & -- \\
\hline \multirow[t]{2}{*}{4} & $10-30-96$ & -- & -- & - & - & -- & - & - & - & -- & - \\
\hline & $05-13-97$ & -- & - & -- & -- & -- & -- & -- & -- & -- & - \\
\hline \multirow[t]{4}{*}{25} & $07-24-97$ & 14 & 2.8 & 5.8 & 1.5 & 49 & 11 & 1.7 & $<.10$ & 22 & 88 \\
\hline & $09-22-97$ & 17 & 3.7 & 7.5 & 1.5 & 57 & 14 & 2.1 & $<.10$ & 21 & 102 \\
\hline & $02-11-98$ & 16 & 3.6 & 7.4 & 1.2 & 53 & 16 & 2.1 & $<.10$ & 21 & 100 \\
\hline & $05-05-98$ & 7.2 & 1.5 & 3.2 & 1.1 & 24 & 5.8 & .69 & $<.10$ & 14 & 48 \\
\hline \multirow[t]{4}{*}{48} & $07-24-97$ & 14 & 2.8 & 5.3 & 1.4 & 47 & 11 & 1.6 & .11 & 20 & 86 \\
\hline & $09-22-97$ & 18 & 3.8 & 7.0 & 1.5 & 56 & 15 & 1.8 & .13 & 20 & 101 \\
\hline & $02-09-98$ & 17 & 3.6 & 6.9 & 1.3 & 53 & 17 & 2.1 & .11 & 20 & 100 \\
\hline & $05-05-98$ & 7.2 & 1.4 & 3.0 & 1.0 & 23 & 5.9 & .62 & $<.10$ & 14 & 47 \\
\hline \multirow[t]{2}{*}{57} & $10-31-96$ & 32 & 8.9 & 13 & 3.1 & 121 & 25 & 2.6 & .50 & 22 & 180 \\
\hline & $05-13-97$ & 12 & 3.2 & 4.0 & 1.8 & 39 & 13 & $<.1$ & .13 & 17 & 75 \\
\hline
\end{tabular}


Table 2. Water-quality data for streams in the Boulder River watershed, Montana, 1996-99 (Continued)

\begin{tabular}{|c|c|c|c|c|c|c|c|}
\hline $\begin{array}{c}\text { Site } \\
\text { num- } \\
\text { ber } \\
\text { (fig. 2) }\end{array}$ & Date & $\begin{array}{c}\text { Nitrite, } \\
\text { dissolved } \\
\text { (mg/L } \\
\text { as } \mathbf{N})\end{array}$ & $\begin{array}{l}\text { Nitrite } \\
\text { plus } \\
\text { nitrate, } \\
\text { dissolved } \\
\text { (mg/L } \\
\text { as } \mathrm{N})\end{array}$ & $\begin{array}{c}\text { Ammonia, } \\
\text { dissolved } \\
\text { (mg/L } \\
\text { as } \mathbf{N})\end{array}$ & $\begin{array}{c}\text { Ammonia plus } \\
\text { organic } \\
\text { nitrogen, } \\
\text { total } \\
(\mathbf{m g} / \mathbf{L} \\
\text { as } \mathbf{N})\end{array}$ & $\begin{array}{c}\text { Phos- } \\
\text { phorus, } \\
\text { total } \\
\text { (mg/L } \\
\text { as P) }\end{array}$ & $\begin{array}{c}\text { Phos- } \\
\text { phorus, } \\
\text { ortho- } \\
\text { phosphate, } \\
\text { dissolved } \\
\text { (mg/L } \\
\text { as P) }\end{array}$ \\
\hline \multicolumn{8}{|c|}{ BOULDER RIVER } \\
\hline \multirow[t]{4}{*}{1} & $07-23-97$ & $<0.01$ & $<0.05$ & $<0.015$ & -- & -- & 0.020 \\
\hline & $09-22-97$ & $<.01$ & $<.05$ & $<.015$ & $<0.2$ & $<0.010$ & $<.010$ \\
\hline & $02-12-98$ & $<.01$ & .12 & .028 & -- & - & .024 \\
\hline & $05-06-98$ & -- & -- & -- & -- & -- & -- \\
\hline 2 & $09-25-98$ & -- & - & - & .11 & $<.05$ & -- \\
\hline \multirow[t]{18}{*}{3} & $10-18-96$ & -- & - & - & - & -- & -- \\
\hline & $11-01-96$ & -- & -- & - & - & - & -- \\
\hline & $12-03-96$ & $<.01$ & .08 & .030 & -- & -- & .020 \\
\hline & $02-11-97$ & -- & - & -- & - & -- & -- \\
\hline & $04-18-97$ & -- & -- & -- & -- & -- & - \\
\hline & $05-07-97$ & -- & -- & -- & -- & -- & -- \\
\hline & $05-14-97$ & .01 & $<.05$ & $<.015$ & -- & -. & .016 \\
\hline & $05-23-97$ & $<.01$ & $<.05$ & $<.015$ & -- & -- & .014 \\
\hline & $06-02-97$ & -- & -- & -- & -- & -- & - \\
\hline & $06-18-97$ & -- & - & -- & -- & - & -- \\
\hline & $07-17-97$ & $<.01$ & $<.05$ & $<.015$ & - & -- & .026 \\
\hline & $09-23-97$ & $<.01$ & $<.05$ & $<.015$ & $<.2$ & .025 & .020 \\
\hline & $02-10-98$ & $<.01$ & .13 & .052 & -- & -- & .026 \\
\hline & $04-29-98$ & -- & -. & -- & -- & -- & -- \\
\hline & $05-05-98$ & -- & -- & -- & -- & -- & -- \\
\hline & $09-23-98$ & -- & -- & -- & -- & -- & - \\
\hline & $05-25-99$ & -- & - & -- & -- & -- & -- \\
\hline & $09-14-99$ & -- & -- & -- & -- & -- & -- \\
\hline \multirow[t]{2}{*}{4} & $10-30-96$ & -- & - & -- & -- & -- & -- \\
\hline & $05-13-97$ & -- & -- & -- & -- & -- & -- \\
\hline \multirow[t]{4}{*}{25} & $07-24-97$ & $<.01$ & $<.05$ & $<.015$ & -. & -- & .021 \\
\hline & $09-22-97$ & $<.01$ & $<.05$ & $<.015$ & $<.2$ & .022 & .017 \\
\hline & $02-11-98$ & $<.01$ & .13 & .030 & -- & -- & .023 \\
\hline & $05-05-98$ & -- & -. & -- & - & -- & -- \\
\hline \multirow[t]{4}{*}{48} & $07-24-97$ & $<.01$ & $<.05$ & $<.015$ & -- & -- & .021 \\
\hline & $09-22-97$ & $<.01$ & $<.05$ & $<.015$ & $<.2$ & .026 & .019 \\
\hline & $02-09-98$ & $<.01$ & .13 & .038 & - & - & .029 \\
\hline & $05-05-98$ & -- & -- & -- & -- & -- & - \\
\hline \multirow[t]{2}{*}{57} & $10-31-96$ & -- & -- & -- & -- & -- & -- \\
\hline & $05-13-97$ & - & -- & -- & - & - & -- \\
\hline
\end{tabular}


Table 2. Water-quality data for streams in the Boulder River watershed, Montana, 1996-99 (Continued)

\begin{tabular}{|c|c|c|c|c|c|c|c|c|c|c|c|c|}
\hline $\begin{array}{c}\text { Site } \\
\text { num- } \\
\text { ber } \\
\text { (fig. 2) }\end{array}$ & Date & $\begin{array}{l}\text { Alumi- } \\
\text { num, } \\
\text { total } \\
\text { recov- } \\
\text { erable } \\
(\mu \mathrm{g} / \mathrm{L} \text { as } \\
\text { Al) }\end{array}$ & $\begin{array}{l}\text { Alumi- } \\
\text { num, } \\
\text { dis- } \\
\text { solved } \\
(\mu \mathrm{g} / \mathrm{L} \text { as } \\
\text { Al) }\end{array}$ & $\begin{array}{l}\text { Anti- } \\
\text { mony, } \\
\text { dis- } \\
\text { solved } \\
(\mu \mathrm{g} / \mathrm{L} \text { as } \\
\text { Sb) }\end{array}$ & $\begin{array}{l}\text { Arsenic, } \\
\text { total } \\
\text { recov- } \\
\text { erable } \\
(\mu \mathrm{g} / \mathrm{L} \text { as } \\
\text { As })\end{array}$ & $\begin{array}{c}\text { Arsenic, } \\
\text { dis- } \\
\text { solved } \\
(\mu \mathrm{g} / \mathrm{L} \text { as } \\
\text { As })\end{array}$ & $\begin{array}{c}\text { Barium, } \\
\text { dis- } \\
\text { solved } \\
(\mu \mathrm{g} / \mathrm{L} \text { as } \\
\text { Ba })\end{array}$ & $\begin{array}{l}\text { Beryl- } \\
\text { lium, } \\
\text { dis- } \\
\text { solved } \\
(\mu \mathrm{g} / \mathrm{L} \text { as } \\
\text { Be) }\end{array}$ & $\begin{array}{c}\text { Cad- } \\
\text { mium, } \\
\text { total } \\
\text { recov- } \\
\text { erable } \\
(\mu \mathrm{g} / \mathrm{L} \text { as } \\
\text { Cd })\end{array}$ & $\begin{array}{c}\text { Cad- } \\
\text { mium, } \\
\text { dis- } \\
\text { solved } \\
(\mu \mathrm{g} / \mathrm{L} \text { as } \\
\text { Cd })\end{array}$ & $\begin{array}{c}\text { Chro- } \\
\text { mium, } \\
\text { total } \\
\text { recov- } \\
\text { erable } \\
(\mu \mathrm{g} / \mathrm{L} \text { as } \\
\text { Cr })\end{array}$ & $\begin{array}{c}\text { Chro- } \\
\text { mium, } \\
\text { dis- } \\
\text { solved } \\
\text { ( } \mu \mathrm{g} / \mathrm{L} \text { as } \\
\text { Cr) }\end{array}$ \\
\hline \multicolumn{13}{|c|}{ BOULDER RIVER } \\
\hline \multirow[t]{4}{*}{1} & $07-23-97$ & 110 & 6.4 & $<1$ & 5 & 4 & 31 & $<1$ & $<1$ & $<.1$ & $<1$ & $<1$ \\
\hline & $09-22-97$ & 30 & 1.9 & $<1$ & 3 & 2 & 38 & $<1$ & $<1$ & $<.1$ & -- & $<1$ \\
\hline & $02-12-98$ & 30 & 3.9 & $<1$ & 2 & 2 & 37 & $<1$ & $<1$ & $<.3$ & -- & 1 \\
\hline & $05-06-98$ & 290 & 26 & $<1$ & 3 & 2 & 17 & $<1$ & $<1$ & $<.3$ & -- & $<1$ \\
\hline 2 & $09-25-98$ & 20 & 2.9 & $<1$ & 1 & 1 & 32 & $<1$ & $<1$ & $<.3$ & -- & $<1$ \\
\hline \multirow[t]{18}{*}{3} & $10-18-96$ & -- & 5.5 & $<1$ & 2 & 3 & 16 & $<1$ & $<1$ & $<.1$ & $<1$ & $<1$ \\
\hline & $11-01-96$ & -- & 9.5 & $<1$ & 3 & 2 & 17 & $<1$ & $<1$ & $<.1$ & -- & $<1$ \\
\hline & $12-03-96$ & -- & 1.5 & $<1$ & 3 & 2 & 17 & $<1$ & $<1$ & $<, 1$ & $<1$ & $<1$ \\
\hline & $02-11-97$ & -- & 1.5 & $<1$ & 3 & 2 & 20 & $<1$ & $<1$ & $<.1$ & -- & 1 \\
\hline & $04-18-97$ & -- & 4.2 & $<1$ & 5 & 3 & 17 & $<1$ & $<1$ & $<.1$ & -- & 1 \\
\hline & $05-07-97$ & 970 & 426 & $<1$ & 6 & 4 & 24 & $<1$ & $<1$ & $<.1$ & -- & $<1$ \\
\hline & $05-14-97$ & 1,190 & 14 & $<1$ & 6 & 3 & 13 & $<1$ & $<1$ & $<.1$ & 1 & $<1$ \\
\hline & $05-23-97$ & 470 & 42 & $<1$ & 4 & 3 & 11 & $<1$ & $<1$ & $<.1$ & $<1$ & $<1$ \\
\hline & $06-02-97$ & 560 & 24 & $<1$ & 6 & 4 & 10 & $<1$ & $<1$ & $<.1$ & -- & $<1$ \\
\hline & $06-18-97$ & 550 & 12 & $<1$ & 6 & 5 & 15 & $<1$ & $<1$ & $<.1$ & -- & $<1$ \\
\hline & $07-17-97$ & -- & 8.9 & $<1$ & 5 & 3 & 18 & $<1$ & $<1$ & $<.1$ & - & 1 \\
\hline & $09-23-97$ & 70 & 1.8 & $<1$ & 4 & 3 & 18 & $<1$ & $<1$ & $<.1$ & -- & $<1$ \\
\hline & $02-10-98$ & 150 & 2.0 & $<1$ & 2 & 2 & 17 & $<1$ & $<1$ & $<.3$ & -- & 1 \\
\hline & $04-29-98$ & 460 & 9.0 & $<1$ & 4 & 3 & 16 & $<1$ & $<1$ & $<.3$ & -. & $<1$ \\
\hline & $05-05-98$ & 490 & 19 & $<1$ & 3 & 3 & 13 & $<1$ & $<1$ & $<.3$ & -- & $<1$ \\
\hline & $09-23-98$ & 40 & 2.7 & $<1$ & 4 & 4 & 15 & $<1$ & $<1$ & $<.3$ & -- & $<1$ \\
\hline & $05-25-99$ & 1,190 & 23 & -- & 7 & 4 & -- & -- & $<1$ & $<.3$ & -- & -- \\
\hline & $09-14-99$ & 49 & 4.9 & -- & 5 & 4 & -- & -- & $<1$ & $<.3$ & -- & -- \\
\hline \multirow[t]{2}{*}{4} & $10-30-96$ & -- & -- & -- & -- & - & -- & -- & -- & -- & -- & $-\cdot$ \\
\hline & $05-13-97$ & -- & - & -- & - & - & -- & -- & -- & -- & - & - \\
\hline \multirow[t]{4}{*}{25} & $07-24-97$ & 130 & 12 & $<1$ & -- & 5 & 20 & $<1$ & $<1$ & .1 & -- & 2 \\
\hline & $09-22-97$ & 60 & 9.7 & $<1$ & 5 & 4 & 19 & $<1$ & $<1$ & .2 & - & $<1$ \\
\hline & $02-11-98$ & 40 & 5.6 & $<1$ & 3 & 3 & 18 & $<1$ & $<1$ & $<.3$ & -- & 1 \\
\hline & $05-05-98$ & 610 & 34 & $<1$ & 7 & 4 & 13 & $<1$ & $<1$ & $<.3$ & -. & $<1$ \\
\hline \multirow[t]{4}{*}{48} & $07-24-97$ & 120 & 15 & $<1$ & 7 & 5 & 19 & $<1$ & $<1$ & .5 & -- & 1 \\
\hline & $09-22-97$ & 60 & 10 & $<1$ & 5 & 4 & 20 & $<1$ & $<1$ & .7 & -- & $<1$ \\
\hline & 02-09-98 & 40 & 5.6 & $<1$ & 3 & 3 & 19 & $<1$ & $<1$ & .6 & - & $<1$ \\
\hline & $05-05-98$ & 530 & 35 & $<1$ & 9 & 3 & 12 & $<1$ & $<1$ & .3 & -- & $<1$ \\
\hline \multirow[t]{2}{*}{57} & $10-31-96$ & -- & $<1$ & $<1$ & $<1$ & $<1$ & 37 & $<1$ & $<1$ & $<.1$ & $<1$ & 1 \\
\hline & $05-13-97$ & 1,470 & 4.2 & $<1$ & 3 & 1 & 21 & $<1$ & $<1$ & $<.1$ & 1 & $<1$ \\
\hline
\end{tabular}


Table 2. Water-quality data for streams in the Boulder River watershed, Montana, 1996-99 (Continued)

\begin{tabular}{|c|c|c|c|c|c|c|c|c|c|c|c|}
\hline $\begin{array}{c}\text { Site } \\
\text { num- } \\
\text { ber } \\
\text { (fig. 2) }\end{array}$ & Date & $\begin{array}{c}\text { Cobalt, } \\
\text { dis- } \\
\text { solved } \\
(\mu \mathrm{g} / \mathrm{L} \text { as } \\
\text { Co })\end{array}$ & $\begin{array}{l}\text { Copper, } \\
\text { total recov- } \\
\text { erable } \\
(\mu \mathrm{g} / \mathrm{L} \text { as } \\
\text { Cu })\end{array}$ & $\begin{array}{c}\text { Copper, } \\
\text { dis- } \\
\text { solved } \\
(\mu \mathrm{g} / \mathrm{L} \text { as } \\
\mathrm{Cu})\end{array}$ & $\begin{array}{c}\text { Iron, } \\
\text { total } \\
\text { recov- } \\
\text { erable } \\
(\mu \mathrm{g} / \mathrm{L} \text { as } \\
\text { Fe })\end{array}$ & $\begin{array}{c}\text { Iron, } \\
\text { dis- } \\
\text { solved } \\
(\mu \mathrm{g} / \mathrm{L} \text { as } \\
\text { Fe })\end{array}$ & $\begin{array}{c}\text { Lead, } \\
\text { total } \\
\text { recov- } \\
\text { erable } \\
(\mu \mathrm{g} / \mathrm{L} \text { as } \\
\text { Pb })\end{array}$ & $\begin{array}{c}\text { Lead, } \\
\text { dis- } \\
\text { solved } \\
(\mu \mathrm{g} / \mathrm{L} \text { as } \\
\mathrm{Pb})\end{array}$ & $\begin{array}{c}\text { Lithium, } \\
\text { dis- } \\
\text { solved } \\
(\mu \mathrm{g} / \mathrm{L} \text { as } \\
\text { Li) }\end{array}$ & $\begin{array}{c}\text { Manga- } \\
\text { nese, } \\
\text { total } \\
\text { recov- } \\
\text { erable } \\
(\mu \mathrm{g} / \mathrm{L} \text { as } \\
\text { Mn })\end{array}$ & $\begin{array}{c}\text { Manga- } \\
\text { nese, } \\
\text { dis- } \\
\text { solved } \\
(\mu \mathrm{g} / \mathrm{L} \text { as } \\
\text { Mn })\end{array}$ \\
\hline \multicolumn{12}{|c|}{ BOULDER RIVER } \\
\hline \multirow[t]{4}{*}{1} & $07-23-97$ & $<1$ & 3 & 1 & 230 & 72 & $<1$ & $<1$ & 5 & 20 & 8 \\
\hline & $09-22-97$ & $<1$ & 1 & 1 & 160 & 45 & $<1$ & $<1$ & -- & $<10$ & 4 \\
\hline & $02-12-98$ & $<1$ & 2 & $<1$ & 140 & 36 & $<1$ & $<1$ & -- & $<10$ & 3 \\
\hline & $05-06-98$ & $<1$ & 3 & 2 & 360 & 73 & $<1$ & $<1$ & -- & 18 & 4 \\
\hline 2 & $09-25-98$ & $<1$ & $<1$ & $<1$ & 240 & 110 & $<1$ & $<1$ & - & 11 & 5 \\
\hline \multirow[t]{18}{*}{3} & $10-18-96$ & $<1$ & 1 & 2 & 230 & 120 & $<1$ & $<1$ & 6 & 10 & 8 \\
\hline & $11-01-96$ & $<1$ & 2 & 2 & 230 & 120 & $<1$ & $<1$ & $<4$ & 20 & 12 \\
\hline & $12-03-96$ & $<1$ & $<1$ & $<1$ & 280 & 65 & $<1$ & $<1$ & 6 & 20 & 11 \\
\hline & $02-11-97$ & $<1$ & 1 & 2 & 430 & 150 & $<1$ & $<1$ & -- & 20 & 16 \\
\hline & $04-18-97$ & $<1$ & 4 & 2 & 990 & 320 & $<1$ & $<1$ & -. & 90 & 68 \\
\hline & $05-07-97$ & $<1$ & 5 & 5 & 970 & 470 & $<1$ & $<1$ & - & 43 & 26 \\
\hline & $05-14-97$ & $<1$ & 16 & 10 & 1,200 & 85 & 3 & $<1$ & $<4$ & 47 & 8 \\
\hline & $05-23-97$ & $<1$ & 10 & 4 & 490 & 100 & $<1$ & $<1$ & $<4$ & 20 & 5 \\
\hline & $06-02-97$ & $<1$ & 7 & 4 & 1,100 & 120 & 1 & $<1$ & -- & 40 & 7 \\
\hline & $06-18-97$ & $<1$ & 4 & 3 & 840 & 150 & 1 & $<1$ & -- & 42 & 11 \\
\hline & $07-17-97$ & $<1$ & 4 & 3 & 710 & 180 & 1 & $<1$ & -- & 48 & 13 \\
\hline & $09-23-97$ & $<1$ & 1 & 2 & 360 & 100 & $<1$ & $<1$ & -- & 23 & 12 \\
\hline & $02-10-98$ & $<1$ & 2 & $<1$ & 470 & 88 & $<1$ & $<1$ & -- & 29 & 14 \\
\hline & $04-29-98$ & $<1$ & 4 & 3 & 850 & 190 & $<1$ & $<1$ & -- & 45 & 8 \\
\hline & $05-05-98$ & $<1$ & 5 & 3 & 820 & 150 & 2 & $<1$ & -- & 35 & 8 \\
\hline & $09-23-98$ & $<1$ & 5 & 2 & 290 & 140 & $<1$ & $<1$ & -- & 15 & 9 \\
\hline & $05-25-99$ & -- & 7 & 3 & 1,600 & 98 & 2 & $<1$ & -- & 97 & 17 \\
\hline & $09-14-99$ & -- & 1 & 2 & 290 & 90 & $<1$ & $<1$ & -- & 12 & 8 \\
\hline \multirow[t]{2}{*}{4} & $10-30-96$ & -- & - & -- & -- & -- & -. & -- & -- & -- & -- \\
\hline & $05-13-97$ & -- & -- & -- & -- & -- & -- & - & -- & -. & - \\
\hline \multirow[t]{4}{*}{25} & $07-24-97$ & $<1$ & 10 & 9 & 400 & 120 & $<1$ & $<1$ & - & 26 & 14 \\
\hline & $09-22-97$ & $<1$ & 11 & 10 & 310 & 130 & $<1$ & $<1$ & -- & 99 & 14 \\
\hline & $02-11-98$ & $<1$ & 8 & 6 & 270 & 64 & $<1$ & $<1$ & -- & 19 & 14 \\
\hline & $05-05-98$ & $<1$ & 9 & 6 & 790 & 140 & 3 & $<1$ & -- & 45 & 13 \\
\hline \multirow[t]{4}{*}{48} & $07-24-97$ & $<1$ & 15 & 12 & 340 & 140 & $<1$ & $<1$ & -- & 21 & 14 \\
\hline & $09-22-97$ & $<1$ & 14 & 12 & 250 & 120 & $<1$ & $<1$ & -- & 20 & 17 \\
\hline & $02-09-98$ & $<1$ & 9 & 7 & 230 & 66 & $<1$ & $<1$ & -- & 18 & 14 \\
\hline & $05-05-98$ & $<1$ & 16 & 11 & 790 & 140 & 5 & $<1$ & -- & 49 & 15 \\
\hline \multirow[t]{2}{*}{57} & $10-31-96$ & $<1$ & $<1$ & $<1$ & 60 & 8 & $<1$ & $<1$ & 21 & 10 & 4 \\
\hline & $05-13-97$ & $<1$ & 7 & 2 & 3,100 & 48 & 4 & $<1$ & 4 & 210 & 11 \\
\hline
\end{tabular}


Table 2. Water-quality data for streams in the Boulder River watershed, Montana, 1996-99 (Continued)

\begin{tabular}{|c|c|c|c|c|c|c|c|c|c|c|c|}
\hline $\begin{array}{c}\text { Site } \\
\text { num- } \\
\text { ber } \\
\text { (fig. 2) }\end{array}$ & Date & $\begin{array}{c}\text { Mercury, } \\
\text { total } \\
\text { recov- } \\
\text { erable } \\
(\mu \mathrm{g} / \mathrm{L} \text { as } \\
\mathrm{Hg})\end{array}$ & $\begin{array}{l}\text { Mercury, } \\
\text { dis- } \\
\text { solved } \\
(\mu \mathrm{g} / \mathrm{L} \text { as } \\
\mathrm{Hg})\end{array}$ & $\begin{array}{c}\text { Molyb- } \\
\text { denum, } \\
\text { dis- } \\
\text { solved } \\
(\mu \mathrm{g} / \mathrm{L} \text { as } \\
\text { Mo) }\end{array}$ & $\begin{array}{c}\text { Nickel, } \\
\text { total } \\
\text { recov- } \\
\text { erable } \\
(\mu \mathrm{g} / \mathrm{L} \text { as } \\
\mathrm{Ni})\end{array}$ & $\begin{array}{c}\text { Nickel, } \\
\text { dis- } \\
\text { solved } \\
(\mu \mathrm{g} / \mathrm{L} \text { as } \\
\mathrm{Ni})\end{array}$ & $\begin{array}{c}\text { Silver, } \\
\text { total } \\
\text { recov- } \\
\text { erable } \\
(\mu \mathrm{g} / \mathrm{L} \text { as } \\
\mathrm{Ag})\end{array}$ & $\begin{array}{l}\text { Silver, } \\
\text { dis- } \\
\text { solved } \\
(\mu \mathrm{g} / \mathrm{L} \text { as } \\
\mathrm{Ag})\end{array}$ & $\begin{array}{c}\text { Uranium, } \\
\text { dis- } \\
\text { solved } \\
(\mu \mathrm{g} / \mathrm{L} \text { as } \\
\mathrm{U})\end{array}$ & $\begin{array}{c}\text { Zinc, } \\
\text { total } \\
\text { recov- } \\
\text { erable } \\
(\mu \mathrm{g} / \mathrm{L} \text { as } \\
\mathrm{Zn})\end{array}$ & $\begin{array}{c}\text { Zinc, } \\
\text { dis- } \\
\text { solved } \\
(\mu \mathrm{g} / \mathrm{L} \text { as } \\
\mathrm{Zn})\end{array}$ \\
\hline
\end{tabular}

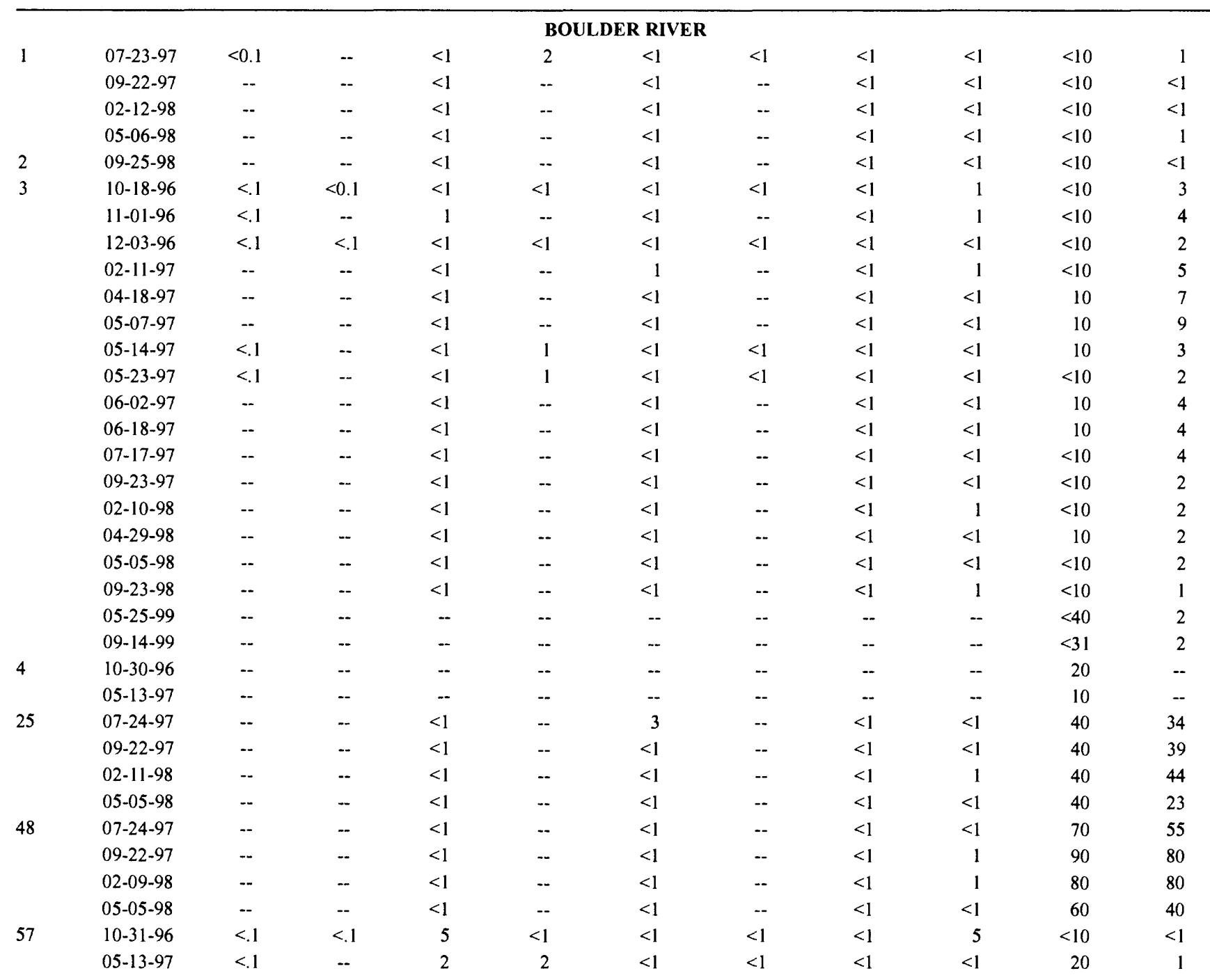


Table 2. Water-quality data for streams in the Boulder River watershed, Montana, 1996-99 (Continued)

\begin{tabular}{|c|c|c|c|c|c|c|c|c|c|c|}
\hline $\begin{array}{c}\text { Site } \\
\text { num- } \\
\text { ber } \\
\text { (fig. 2) }\end{array}$ & Station name & Date & Time & $\begin{array}{c}\text { Dis- } \\
\text { charge, } \\
\text { instanta- } \\
\text { neous } \\
\left(\mathrm{ft}^{3} / \mathbf{s}\right)\end{array}$ & $\begin{array}{c}\text { Sedi- } \\
\text { ment, } \\
\text { sus- } \\
\text { pended } \\
\text { (mg/L) }\end{array}$ & $\begin{array}{c}\text { Sedi- } \\
\text { ment, } \\
\text { sus- } \\
\text { pended, } \\
\text { diameter } \\
\text { (percent } \\
\text { finer } \\
\text { than } \\
0.062 \\
\text { mm) }\end{array}$ & $\begin{array}{l}\text { Spe- } \\
\text { cific } \\
\text { con- } \\
\text { duct- } \\
\text { ance, } \\
\text { field } \\
(\mu \mathrm{S} / \mathrm{cm})\end{array}$ & $\begin{array}{c}\text { pH, } \\
\text { field } \\
\text { (stan- } \\
\text { dard } \\
\text { units) }\end{array}$ & $\begin{array}{l}\text { Tem- } \\
\text { pera- } \\
\text { ture, } \\
\text { water } \\
\left({ }^{\circ} \mathrm{C}\right)\end{array}$ & $\begin{array}{c}\text { Hard- } \\
\text { ness } \\
(\mathrm{mg} / \mathrm{L} \\
\text { as } \\
\left.\mathrm{CaCO}_{3}\right)\end{array}$ \\
\hline \multicolumn{11}{|c|}{ BOULDER RIVER--Continued } \\
\hline \multirow[t]{19}{*}{58} & Boulder River below Little Galena Gulch, near & $10-15-96$ & 1115 & 29 & 1 & 81 & 156 & 8.2 & 4.5 & 65 \\
\hline & Boulder & $11-01-96$ & 1200 & 29 & 4 & 68 & 170 & 8.2 & 0.0 & 68 \\
\hline & & $12-04-96$ & 1205 & 35 & 5 & 67 & 155 & 8.1 & 0.0 & 61 \\
\hline & & $02-12-97$ & 1145 & 26 & 3 & 77 & 166 & 7.7 & 0.0 & 65 \\
\hline & & 04-19-97 & 0930 & 187 & 56 & 85 & 102 & 8.0 & 3.5 & 48 \\
\hline & & $05-07-97$ & 1330 & 369 & 24 & 70 & 100 & 7.9 & 6.5 & 37 \\
\hline & & $05-14-97$ & 1330 & 900 & 139 & 21 & 71 & 7.7 & 10.5 & 25 \\
\hline & & $05-23-97$ & 1300 & 960 & 87 & 17 & 58 & 7.6 & 9.5 & 20 \\
\hline & & $06-02-97$ & 1145 & 1,050 & 56 & 36 & 59 & 7.5 & 10.0 & 22 \\
\hline & & $06-18-97$ & 1400 & 635 & 33 & 39 & 83 & 8.0 & 14.0 & 32 \\
\hline & & $07-17-97$ & 1115 & 175 & 15 & 65 & 118 & 8.1 & 15.5 & 47 \\
\hline & & $09-23-97$ & 1420 & 36 & 3 & 89 & 161 & 8.2 & 12.5 & 64 \\
\hline & & $02-09-98$ & 0915 & 27 & 3 & 79 & 158 & 7.8 & 0.0 & 62 \\
\hline & & $04-29-98$ & 1400 & 256 & 36 & 47 & 96 & 8.2 & 7.5 & 38 \\
\hline & & $05-05-98$ & 1510 & 547 & 32 & 50 & 66 & 7.7 & 10.0 & 25 \\
\hline & & $06-18-98$ & 1000 & 614 & 43 & 37 & 73 & 7.6 & 6.5 & 29 \\
\hline & & $09-23-98$ & 1245 & 30 & 6 & 45 & 154 & 8.5 & 10.0 & 64 \\
\hline & & $05-25-99$ & 1525 & 602 & 52 & 59 & 58 & 8.0 & 11.0 & 19 \\
\hline & & 09-14-99 & 1230 & 17 & 2 & 75 & 165 & 8.0 & 13.0 & 64 \\
\hline \multirow[t]{2}{*}{59} & Boulder River near Boulder & $11-01-96$ & 1240 & 38 & 12 & 58 & 203 & 7.6 & 3.5 & 76 \\
\hline & & $05-24-97$ & 0930 & 1,420 & 305 & 29 & 59 & 7.7 & 6.5 & 21 \\
\hline \multirow[t]{15}{*}{60} & Little Boulder River above North Fork, near Boulder & $10-31-96$ & 0945 & 2.4 & 2 & 62 & 129 & 8.0 & 0.0 & 51 \\
\hline & & $05-13-97$ & 1000 & 69 & 58 & 45 & 53 & 7.2 & 6.0 & 19 \\
\hline & & $05-23-97$ & 1700 & 94 & 13 & 60 & 52 & 7.8 & 8.5 & 19 \\
\hline & & $07-17-97$ & 1345 & 11 & 3 & 71 & 101 & 8.1 & 15.0 & 37 \\
\hline & & $09-25-97$ & 1515 & 3.7 & 5 & 63 & 123 & 8.1 & 10.0 & 53 \\
\hline & & $07-21-98$ & 1545 & 6.3 & 2 & 78 & 114 & 8.1 & 18.0 & 48 \\
\hline & & $07-22-98$ & 0900 & -- & -- & -- & 114 & 8.0 & 13.0 & -- \\
\hline & & $07-23-98$ & 0805 & -- & -- & -- & 114 & 8.2 & 13.0 & -- \\
\hline & & $07-24-98$ & 0825 & -- & -- & -- & 113 & 8.1 & 14.0 & -- \\
\hline & & $07-25-98$ & 1415 & -- & -- & -- & 114 & 8.2 & 16.0 & -- \\
\hline & & $09-23-98$ & 0915 & 3.9 & 2 & 71 & 115 & 7.6 & 5.5 & 51 \\
\hline & & $08-02-99$ & 1830 & 2.6 & 1 & 87 & 134 & 8.1 & 16.5 & 56 \\
\hline & & $08-03-99$ & -- & -- & -- & -- & 134 & 8.1 & 16.0 & -- \\
\hline & & 08-04-99 & 1700 & -- & -- & -. & - & -- & -- & -- \\
\hline & & 08-05-99 & 1330 & -- & -- & - & 105 & 7.9 & 15.0 & - \\
\hline \multirow[t]{2}{*}{61} & Boulder River at County Bridge, near Boulder & $11-01-96$ & 1415 & 48 & 10 & 89 & 196 & 8.0 & 1.0 & 73 \\
\hline & & $05-24-97$ & 1200 & 1,350 & 368 & 44 & 71 & 7.7 & 9.5 & 25 \\
\hline \multirow[t]{2}{*}{62} & Boulder River near Cardwell & $11-02-96$ & 0945 & 101 & 11 & 78 & 332 & 8.4 & 2.5 & 130 \\
\hline & & $05-24-97$ & 1530 & 1,030 & 121 & 54 & 128 & 8.0 & 12.0 & 50 \\
\hline
\end{tabular}


Table 2. Water-quality data for streams in the Boulder River watershed, Montana, 1996-99 (Continued)

\begin{tabular}{|c|c|c|c|c|c|c|c|c|c|c|c|}
\hline $\begin{array}{c}\text { Site } \\
\text { num- } \\
\text { ber } \\
\text { (fig. 2) }\end{array}$ & Date & $\begin{array}{c}\text { Cal- } \\
\text { cium, } \\
\text { dissolved } \\
(\mathrm{mg} / \mathrm{L} \\
\text { as Ca) }\end{array}$ & $\begin{array}{l}\text { Magne- } \\
\text { sium, } \\
\text { dissolved } \\
\text { (mg/L } \\
\text { as } \mathbf{M g )}\end{array}$ & $\begin{array}{c}\text { Sodium, } \\
\text { dissolved } \\
(\mathbf{m g} / \mathbf{L} \\
\text { as } \mathrm{Na})\end{array}$ & $\begin{array}{l}\text { Potas- } \\
\text { sium, } \\
\text { dissolved } \\
\text { (mg/L } \\
\text { as K) }\end{array}$ & $\begin{array}{c}\mathrm{ANC}, \\
\mathrm{lab} \\
(\mathrm{mg} / \mathrm{L} \\
\left.\text { as } \mathrm{CaCO}_{3}\right)\end{array}$ & $\begin{array}{c}\text { Sulfate, } \\
\text { dissolved } \\
(\mathrm{mg} / \mathrm{L} \\
\left.\text { as } \mathrm{SO}_{4}\right)\end{array}$ & $\begin{array}{c}\text { Chloride, } \\
\text { dissolved } \\
(\mathrm{mg} / \mathrm{L} \\
\text { as } \mathrm{Cl})\end{array}$ & $\begin{array}{c}\text { Fluoride, } \\
\text { dissolved } \\
(\mathbf{m g} / \mathbf{L} \\
\text { as F) }\end{array}$ & $\begin{array}{c}\text { Silica, } \\
\text { dissolved } \\
(\mathbf{m g} / \mathbf{L} \\
\left.\text { as } \mathrm{SiO}_{2}\right)\end{array}$ & $\begin{array}{l}\text { Dis- } \\
\text { solved } \\
\text { solids, } \\
\text { calcu- } \\
\text { lated } \\
(\mathrm{mg} / \mathrm{L})\end{array}$ \\
\hline
\end{tabular}

\begin{tabular}{|c|c|c|c|c|c|c|c|c|c|c|c|}
\hline \multirow[b]{2}{*}{58} & \multicolumn{11}{|c|}{ BOULDER RIVER--Continued } \\
\hline & $10-15-96$ & 19 & 4.3 & 7.0 & 1.6 & 59 & 21 & 1.9 & .10 & 18 & 109 \\
\hline & $11-01-96$ & 20 & 4.3 & 7.6 & 1.5 & 60 & 24 & 1.9 & .10 & 21 & 117 \\
\hline & $12-04-96$ & 18 & 3.9 & 6.9 & 1.4 & 55 & 21 & 1.5 & .10 & 20 & 107 \\
\hline & $02-12-97$ & 19 & 4.2 & -- & -- & -- & -- & -- & - & -- & -- \\
\hline & $04-19-97$ & 14 & 3.1 & 4.9 & 1.8 & 44 & 15 & 1.8 & $<.10$ & 15 & 82 \\
\hline & $05-07-97$ & 11 & 2.5 & 4.4 & 1.4 & 33 & 12 & 1.4 & $<.10$ & 17 & 70 \\
\hline & $05-14-97$ & 7.5 & 1.5 & 3.4 & 1.1 & 23 & 8.1 & .78 & $<.10$ & 16 & 52 \\
\hline & $05-23-97$ & 6.0 & 1.2 & 2.8 & .90 & 19 & 5.7 & .47 & $<.10$ & 16 & 45 \\
\hline & $06-02-97$ & 6.4 & 1.4 & 2.9 & .98 & 21 & 6.1 & .58 & $<.10$ & 15 & 47 \\
\hline & $06-18-97$ & 9.7 & 2.0 & 4.0 & 1.1 & 32 & 6.1 & .76 & $<.10$ & 18 & 62 \\
\hline & $07-17-97$ & 14 & 2.7 & 5.2 & 1.4 & 44 & 12 & 1.3 & $<.10$ & 20 & 83 \\
\hline & $09-23-97$ & 19 & 4.2 & 7.0 & 1.5 & 59 & 18 & 2.0 & .14 & 19 & 107 \\
\hline & $02-09-98$ & 18 & 4.1 & 6.9 & 1.4 & 56 & 19 & 2.2 & $<.10$ & 20 & 106 \\
\hline & $04-29-98$ & 11 & 2.3 & 4.1 & 1.4 & 37 & 9.4 & 1.3 & $<.10$ & 15 & 67 \\
\hline & $05-05-98$ & 7.4 & 1.6 & 3.0 & 1.0 & 24 & 6.3 & .74 & $<.10$ & 14 & 48 \\
\hline & $06-18-98$ & 8.5 & 1.8 & 3.4 & .85 & 28 & 6.0 & .59 & .11 & 15 & 54 \\
\hline & $09-23-98$ & 19 & 4.1 & 7.2 & 1.6 & 60 & 17 & 1.9 & .12 & 19 & 106 \\
\hline & $05-25-99$ & 5.7 & 1.2 & 2.6 & .95 & 19 & 5.4 & .66 & -- & -- & -- \\
\hline & $09-14-99$ & 19 & 4.1 & 7.6 & 1.7 & 59 & 20 & 2.1 & -- & -- & -- \\
\hline \multirow[t]{2}{*}{59} & $11-01-96$ & 22 & 5.2 & 8.5 & 1.7 & 68 & 27 & 2.9 & .10 & 21 & 130 \\
\hline & $05-24-97$ & 6.2 & 1.3 & 2.8 & .96 & 19 & 6.7 & .61 & $<.10$ & 14 & 45 \\
\hline \multirow[t]{15}{*}{60} & $10-31-96$ & 15 & 3.2 & 5.0 & 1.3 & 51 & 13 & .80 & $<.10$ & 23 & 92 \\
\hline & $05-13-97$ & 5.7 & 1.2 & 2.3 & .87 & 18 & 5.6 & $<.1$ & $<.10$ & 13 & 40 \\
\hline & $05-23-97$ & 5.7 & 1.2 & 2.4 & .77 & 18 & 5.1 & .45 & $<.10$ & 13 & 40 \\
\hline & $07-17-97$ & 11 & 2.2 & 3.6 & 1.1 & 42 & 7.7 & .53 & $<.10$ & 19 & 71 \\
\hline & $09-25-97$ & 16 & 3.3 & 4.6 & 1.2 & 51 & 10 & .71 & $<.10$ & 19 & 86 \\
\hline & $07-21-98$ & 14 & 2.9 & 4.4 & 1.2 & 48 & 7.9 & .76 & $<.10$ & 14 & 75 \\
\hline & $07-22-98$ & -- & -- & -- & - & - & -- & -- & - & -- & -- \\
\hline & $07-23-98$ & -- & -- & -- & - & - & -- & -- & - & - & -- \\
\hline & $07-24-98$ & -- & -- & -- & -- & -- & -- & -- & - & -- & -- \\
\hline & $07-25-98$ & -- & -- & -- & $-\cdot$ & - & -- & - & - & - & - \\
\hline & $09-23-98$ & 15 & 3.2 & 4.9 & 1.3 & 52 & 9.9 & .72 & $<.10$ & 20 & 86 \\
\hline & $08-02-99$ & 17 & 3.5 & 5.1 & 1.5 & 57 & 9.9 & .72 & - & -- & - \\
\hline & $08-03-99$ & -- & -- & -- & -- & -- & -- & - & - & -- & -- \\
\hline & $08-04-99$ & -- & -- & -- & -- & -- & -- & -- & -- & -- & -- \\
\hline & $08-05-99$ & -- & -- & -- & -- & -- & -- & - & -- & -- & -- \\
\hline \multirow[t]{2}{*}{61} & $11-01-96$ & 21 & 5.0 & 10 & 1.6 & 72 & 23 & 3.5 & .30 & 21 & 129 \\
\hline & $05-24-97$ & 7.5 & 1.6 & 3.4 & 1.1 & 23 & 8.4 & .80 & $<.10$ & 15 & 52 \\
\hline \multirow[t]{2}{*}{62} & $11-02-96$ & 37 & 10 & 14 & 1.9 & 136 & 32 & 5.3 & .40 & 21 & 203 \\
\hline & $05-24-97$ & 15 & 3.3 & 5.5 & 1.6 & 47 & 12 & 1.5 & .13 & 17 & 84 \\
\hline
\end{tabular}


Table 2. Water-quality data for streams in the Boulder River watershed, Montana, 1996-99 (Continued)

\begin{tabular}{|c|c|c|c|c|c|c|c|}
\hline $\begin{array}{c}\text { Site } \\
\text { num- } \\
\text { ber } \\
\text { (fig. 2) }\end{array}$ & Date & $\begin{array}{c}\text { Nitrite, } \\
\text { dissolved } \\
\text { (mg/L } \\
\text { as } \mathbf{N})\end{array}$ & $\begin{array}{l}\text { Nitrite } \\
\text { plus } \\
\text { nitrate, } \\
\text { dissolved } \\
\text { (mg/L } \\
\text { as } N \text { ) }\end{array}$ & $\begin{array}{c}\text { Ammonia, } \\
\text { dissolved } \\
\text { (mg/L } \\
\text { as } N)\end{array}$ & $\begin{array}{c}\text { Ammonia plus } \\
\text { organic } \\
\text { nitrogen, } \\
\text { total } \\
\text { (mg/L } \\
\text { as } N \text { ) }\end{array}$ & $\begin{array}{c}\text { Phos- } \\
\text { phorus, } \\
\text { total } \\
\text { (mg/L } \\
\text { as P) }\end{array}$ & $\begin{array}{l}\text { Phos- } \\
\text { phorus, } \\
\text { ortho- } \\
\text { phosphate, } \\
\text { dissolved } \\
\text { (mg/L } \\
\text { as P) }\end{array}$ \\
\hline
\end{tabular}

\begin{tabular}{|c|c|c|c|c|c|c|c|}
\hline \multicolumn{8}{|c|}{ BOULDER RIVER-Continued } \\
\hline \multirow[t]{19}{*}{58} & $10-15-96$ & - & -- & -- & -- & -- & -- \\
\hline & $11-01-96$ & - & -- & -- & - & -- & -- \\
\hline & $12-04-96$ & $<.01$ & .10 & .040 & -- & -- & .020 \\
\hline & $02-12-97$ & -- & - & -- & - & -- & -- \\
\hline & $04-19-97$ & -- & -- & -- & -- & -- & - \\
\hline & $05-07-97$ & -- & -- & -- & -- & -- & -- \\
\hline & $05-14-97$ & .01 & $<.05$ & $<.015$ & -- & -- & .011 \\
\hline & $05-23-97$ & $<.01$ & $<.05$ & $<.015$ & -- & -- & $<.010$ \\
\hline & $06-02-97$ & - & -- & - & -- & -- & -- \\
\hline & $06-18-97$ & - & -- & -- & -- & -- & -- \\
\hline & $07-17-97$ & $<.01$ & $<.05$ & $<.015$ & -- & -- & .019 \\
\hline & $09-23-97$ & $<.01$ & $<.05$ & $<.015$ & $<.2$ & .011 & .011 \\
\hline & $02-09-98$ & $<.01$ & .15 & .029 & -- & -- & .021 \\
\hline & $04-29-98$ & -- & -- & -- & -- & -- & - \\
\hline & $05-05-98$ & -- & -- & -- & -- & -- & -- \\
\hline & $06-18-98$ & -- & -- & - & -- & -- & -- \\
\hline & $09-23-98$ & -- & -- & -- & -- & -- & -- \\
\hline & $05-25-99$ & -- & -- & -- & -- & -- & -- \\
\hline & $09-14-99$ & -- & -- & -- & -- & -- & - \\
\hline \multirow[t]{2}{*}{59} & $11-01-96$ & -- & -- & -- & -- & -- & -- \\
\hline & $05-24-97$ & -- & -- & -- & -- & -- & -- \\
\hline \multirow[t]{15}{*}{60} & $10-31-96$ & .03 & .06 & .020 & -- & -- & $<.010$ \\
\hline & $05-13-97$ & $<.01$ & .05 & $<.015$ & -- & -- & $<.010$ \\
\hline & $05-23-97$ & $<.01$ & $<.05$ & $<.015$ & -- & -- & $<.010$ \\
\hline & $07-17-97$ & $<.01$ & $<.05$ & $<.015$ & -- & -- & .017 \\
\hline & $09-25-97$ & $<.01$ & $<.05$ & $<.015$ & $<.2$ & $<.010$ & .010 \\
\hline & $07-21-98$ & $<.01$ & $<.05$ & .030 & -- & -- & .011 \\
\hline & $07-22-98$ & -- & -- & -- & -- & -- & -- \\
\hline & $07-23-98$ & -- & -- & -- & -- & -- & -- \\
\hline & $07-24-98$ & -- & - & -- & -- & -- & -- \\
\hline & $07-25-98$ & -- & -- & -- & -- & -- & -- \\
\hline & $09-23-98$ & -- & -- & -- & $<.1$ & $<.050$ & - \\
\hline & 08-02-99 & -- & -- & -- & -- & -- & -- \\
\hline & 08-03-99 & -- & -- & -- & -- & -- & -- \\
\hline & 08-04-99 & -- & -- & -- & -- & -- & - \\
\hline & $08-05-99$ & -- & -- & -- & - & -- & - \\
\hline \multirow[t]{2}{*}{61} & $11-01-96$ & -- & -- & -- & -- & -- & - \\
\hline & $05-24-97$ & -- & - & -- & -- & -- & -- \\
\hline \multirow[t]{2}{*}{62} & $11-02-96$ & -- & -- & -- & -- & - & - \\
\hline & $05-24-97$ & -- & -- & -- & - & -- & -- \\
\hline
\end{tabular}


Table 2. Water-quality data for streams in the Boulder River watershed, Montana, 1996-99 (Continued)

\begin{tabular}{|c|c|c|c|c|c|c|c|c|c|c|c|c|}
\hline $\begin{array}{c}\text { Site } \\
\text { num- } \\
\text { ber } \\
\text { (fig. 2) }\end{array}$ & Date & $\begin{array}{l}\text { Alumi- } \\
\text { num, } \\
\text { total } \\
\text { recov- } \\
\text { erable } \\
(\mu \mathrm{g} / \mathrm{L} \text { as } \\
\mathrm{Al})\end{array}$ & $\begin{array}{l}\text { Alumi- } \\
\text { num, } \\
\text { dis- } \\
\text { solved } \\
(\mu \mathrm{g} / \mathrm{L} \text { as } \\
\text { Al) }\end{array}$ & $\begin{array}{c}\text { Anti- } \\
\text { mony, } \\
\text { dis- } \\
\text { solved } \\
(\mu \mathrm{g} / \mathrm{L} \text { as } \\
\text { Sb) }\end{array}$ & $\begin{array}{l}\text { Arsenic, } \\
\text { total } \\
\text { recov- } \\
\text { erable } \\
(\mu \mathrm{g} / \mathrm{L} \text { as } \\
\text { As) }\end{array}$ & $\begin{array}{c}\text { Arsenic, } \\
\text { dis- } \\
\text { solved } \\
(\mu \mathrm{g} / \mathrm{L} \text { as } \\
\text { As) }\end{array}$ & $\begin{array}{c}\text { Barium, } \\
\text { dis- } \\
\text { solved } \\
(\mu \mathrm{g} / \mathrm{L} \text { as } \\
\text { Ba })\end{array}$ & $\begin{array}{c}\text { Beryl- } \\
\text { lium, } \\
\text { dis- } \\
\text { solved } \\
(\mu \mathrm{g} / \mathrm{L} \text { as } \\
\text { Be) }\end{array}$ & $\begin{array}{c}\text { Cad- } \\
\text { mium, } \\
\text { total } \\
\text { recov- } \\
\text { erable } \\
(\mu \mathrm{g} / \mathrm{L} \text { as } \\
\text { Cd })\end{array}$ & $\begin{array}{l}\text { Cad- } \\
\text { mium, } \\
\text { dis- } \\
\text { solved } \\
(\mu \mathrm{g} / \mathrm{L} \text { as } \\
\text { Cd) }\end{array}$ & $\begin{array}{c}\text { Chro- } \\
\text { mium, } \\
\text { total } \\
\text { recov- } \\
\text { erable } \\
(\mu \mathrm{g} / \mathrm{L} \text { as } \\
\text { Cr })\end{array}$ & $\begin{array}{l}\text { Chro- } \\
\text { mium, } \\
\text { dis- } \\
\text { solved } \\
(\mu \mathrm{g} / \mathrm{L} \text { as } \\
\text { Cr })\end{array}$ \\
\hline \multicolumn{13}{|c|}{ BOULDER RIVER--Continued } \\
\hline \multirow[t]{19}{*}{58} & $10-15-96$ & -- & 13 & $<1$ & 6 & 5 & 22 & $<1$ & 1 & 1.0 & $<1$ & $<1$ \\
\hline & $11-01-96$ & -- & 12 & $<1$ & 7 & 5 & 22 & $<1$ & 1 & 1.0 & - & $<1$ \\
\hline & $12-04-96$ & -- & 6.5 & $<1$ & 6 & 4 & 20 & $<1$ & $<1$ & .8 & $<1$ & $<1$ \\
\hline & $02-12-97$ & -- & 6.5 & $<1$ & 5 & 4 & 23 & $<1$ & $<1$ & .8 & -- & $<1$ \\
\hline & $04-19-97$ & -- & 7.5 & $<1$ & 13 & 4 & 16 & $<1$ & $<1$ & .2 &.- & $<1$ \\
\hline & $05-07-97$ & 540 & 87 & $<1$ & 8 & 4 & 17 & $<1$ & $<1$ & .2 & -- & $<1$ \\
\hline & $05-14-97$ & 1,340 & 27 & $<1$ & 16 & 4 & 12 & $<1$ & $<1$ & .3 & 1 & $<1$ \\
\hline & $05-23-97$ & 670 & 62 & $<1$ & 12 & 4 & 11 & $<1$ & $<1$ & .4 & $<1$ & $<1$ \\
\hline & $06-02-97$ & 670 & 87 & $<1$ & 12 & 5 & 11 & $<1$ & $<1$ & .3 & -- & $<1$ \\
\hline & $06-18-97$ & 450 & 21 & $<1$ & 12 & 5 & 15 & $<1$ & $<1$ & .3 & - & $<1$ \\
\hline & $07-17-97$ & -- & 20 & $<1$ & 17 & 5 & 19 & $<1$ & $<1$ & .6 & -- & $<1$ \\
\hline & $09-23-97$ & 40 & 8.0 & $<1$ & 7 & 5 & 21 & $<1$ & $<1$ & .7 & -- & $<1$ \\
\hline & $02-09-98$ & 50 & 4.4 & $<1$ & 4 & 3 & 20 & $<1$ & $<1$ & .7 & -- & $<1$ \\
\hline & $04-29-98$ & 360 & 16 & $<1$ & 8 & 3 & 16 & $<1$ & $<1$ & .3 & -- & $<1$ \\
\hline & $05-05-98$ & 560 & 43 & $<1$ & 12 & 4 & 13. & $<1$ & $<1$ & .4 & -- & $<1$ \\
\hline & $06-18-98$ & -- & 46 & $<1$ & 7 & 3 & 13 & $<1$ & $<1$ & .3 & -- & $<1$ \\
\hline & $09-23-98$ & 40 & 5.6 & $<1$ & 6 & 6 & 21 & $<1$ & $<1$ & .7 & - & $<1$ \\
\hline & $05-25-99$ & 820 & 51 & -- & 19 & 5 & -- & -- & $<1$ & $<.3$ & -- & -- \\
\hline & 09-14-99 & 40 & 7.5 & - & 7 & 6 & $\cdots$ & -- & 1 & .9 & -- & -- \\
\hline \multirow[t]{2}{*}{59} & $11-01-96$ & -- & 6.5 & $<1$ & 11 & 7 & 29 & $<1$ & $<1$ & .8 & -- & $<1$ \\
\hline & $05-24-97$ & 2,050 & 68 & $<1$ & 97 & 5 & 12 & $<1$ & 2 & .5 & -- & $<1$ \\
\hline \multirow[t]{15}{*}{60} & $10-31-96$ & $-\cdot$ & 3.5 & $<1$ & 2 & 1 & 11 & $<1$ & $<1$ & $<.1$ & $<1$ & $<1$ \\
\hline & $05-13-97$ & -- & 28 & $<1$ & 7 & 3 & 5.9 & $<1$ & $<1$ & $<.1$ & 1 & $<1$ \\
\hline & $05-23-97$ & -- & 61 & $<1$ & 5 & 3 & 6.7 & $<1$ & $<1$ & $<.1$ & $<1$ & $<1$ \\
\hline & $07-17-97$ & -- & 9.4 & $<1$ & 3 & 2 & 10 & $<1$ & $<1$ & $<.1$ & -- & $<1$ \\
\hline & $09-25-97$ & 40 & 2.9 & $<1$ & 2 & 1 & 11 & $<1$ & $<1$ & $<.1$ & -- & $<1$ \\
\hline & $07-21-98$ & 20 & 7.5 & $<1$ & 3 & 3 & 12 & $<1$ & $<1$ & .3 & -- & $<1$ \\
\hline & $07-22-98$ & -- & -- & -- & -- & -- & -- & -- & -- & $<.1$ & -- & -- \\
\hline & $07-23-98$ & -- & -- & -- & -- & - & -- & - & -- & $<.1$ & -- & - \\
\hline & $07-24-98$ & - & -- & -- & -- & -- & -- & -- & -- & $<.1$ & -- & - \\
\hline & $07-25-98$ & -- & -- & -- & - & -- & -- & -- & -- & $<.1$ & -- & -- \\
\hline & $09-23-98$ & 20 & 5.6 & $<1$ & 2 & 2 & 11 & $<1$ & $<1$ & $<.3$ & -- & $<1$ \\
\hline & $08-02-99$ & e20 & 4.0 & -- & 2 & 2 & -- & -- & $<1$ & $<1$ & -- & -- \\
\hline & $08-03-99$ & -- & -- & -- & - & -- & -- & -- & -- & $<1$ & -- & - \\
\hline & $08-04-99$ & -- & - & -- & - & -- & -- & -- & -- & $<1$ & -- & - \\
\hline & $08-05-99$ & -- & -- & -- & -- & -- & -- & -- & -- & $<1$ & -- & -- \\
\hline \multirow[t]{2}{*}{61} & $11-01-96$ & -- & 2.5 & $<1$ & 14 & 9 & 27 & $<1$ & $<1$ & .5 & -- & $<1$ \\
\hline & $05-24-97$ & 4,100 & 29 & $<1$ & 90 & 8 & 13 & $<1$ & 3 & .6 & -- & $<1$ \\
\hline \multirow[t]{2}{*}{62} & $11-02-96$ & -- & 2.5 & $<1$ & 8 & 7 & 40 & $<1$ & $<1$ & $<.1$ & -- & 1 \\
\hline & $05-24-97$ & 1,890 & 19 & $<1$ & 30 & 15 & 19 & $<1$ & $<1$ & .2 & -- & $<1$ \\
\hline
\end{tabular}


Table 2. Water-quality data for streams in the Boulder River watershed, Montana, 1996-99 (Continued)

\begin{tabular}{|c|c|c|c|c|c|c|c|c|c|c|c|}
\hline $\begin{array}{c}\text { Site } \\
\text { num- } \\
\text { ber } \\
\text { (fig. 2) }\end{array}$ & Date & $\begin{array}{l}\text { Cobalt, } \\
\text { dis- } \\
\text { solved } \\
(\mu \mathrm{g} / \mathrm{L} \text { as } \\
\text { Co })\end{array}$ & $\begin{array}{l}\text { Copper, } \\
\text { total recov- } \\
\text { erable } \\
(\mu \mathrm{g} / \mathrm{L} \text { as } \\
\text { Cu })\end{array}$ & $\begin{array}{l}\text { Copper, } \\
\text { dis- } \\
\text { solved } \\
(\mu \mathrm{g} / \mathrm{L} \text { as } \\
\mathrm{Cu})\end{array}$ & $\begin{array}{c}\text { Iron, } \\
\text { total } \\
\text { recov- } \\
\text { erable } \\
(\mu \mathrm{g} / \mathrm{L} \text { as } \\
\mathrm{Fe})\end{array}$ & $\begin{array}{c}\text { Iron, } \\
\text { dis- } \\
\text { solved } \\
(\mu \mathrm{g} / \mathrm{L} \text { as } \\
\text { Fe })\end{array}$ & $\begin{array}{c}\text { Lead, } \\
\text { total } \\
\text { recov- } \\
\text { erable } \\
(\mu \mathrm{g} / \mathrm{L} \text { as } \\
\mathrm{Pb})\end{array}$ & $\begin{array}{c}\text { Lead, } \\
\text { dis- } \\
\text { solved } \\
(\mu \mathrm{g} / \mathrm{L} \text { as } \\
\mathrm{Pb})\end{array}$ & $\begin{array}{c}\text { Lithium, } \\
\text { dis- } \\
\text { solved } \\
(\mu \mathrm{g} / \mathrm{L} \text { as } \\
\text { Li) }\end{array}$ & $\begin{array}{c}\text { Manga- } \\
\text { nese, } \\
\text { total } \\
\text { recov- } \\
\text { erable } \\
(\mu \mathrm{g} / \mathrm{L} \text { as } \\
\text { Mn) }\end{array}$ & $\begin{array}{l}\text { Manga- } \\
\text { nese, } \\
\text { dis- } \\
\text { solved } \\
(\mu \mathrm{g} / \mathrm{L} \text { as } \\
\mathrm{Mn})\end{array}$ \\
\hline
\end{tabular}

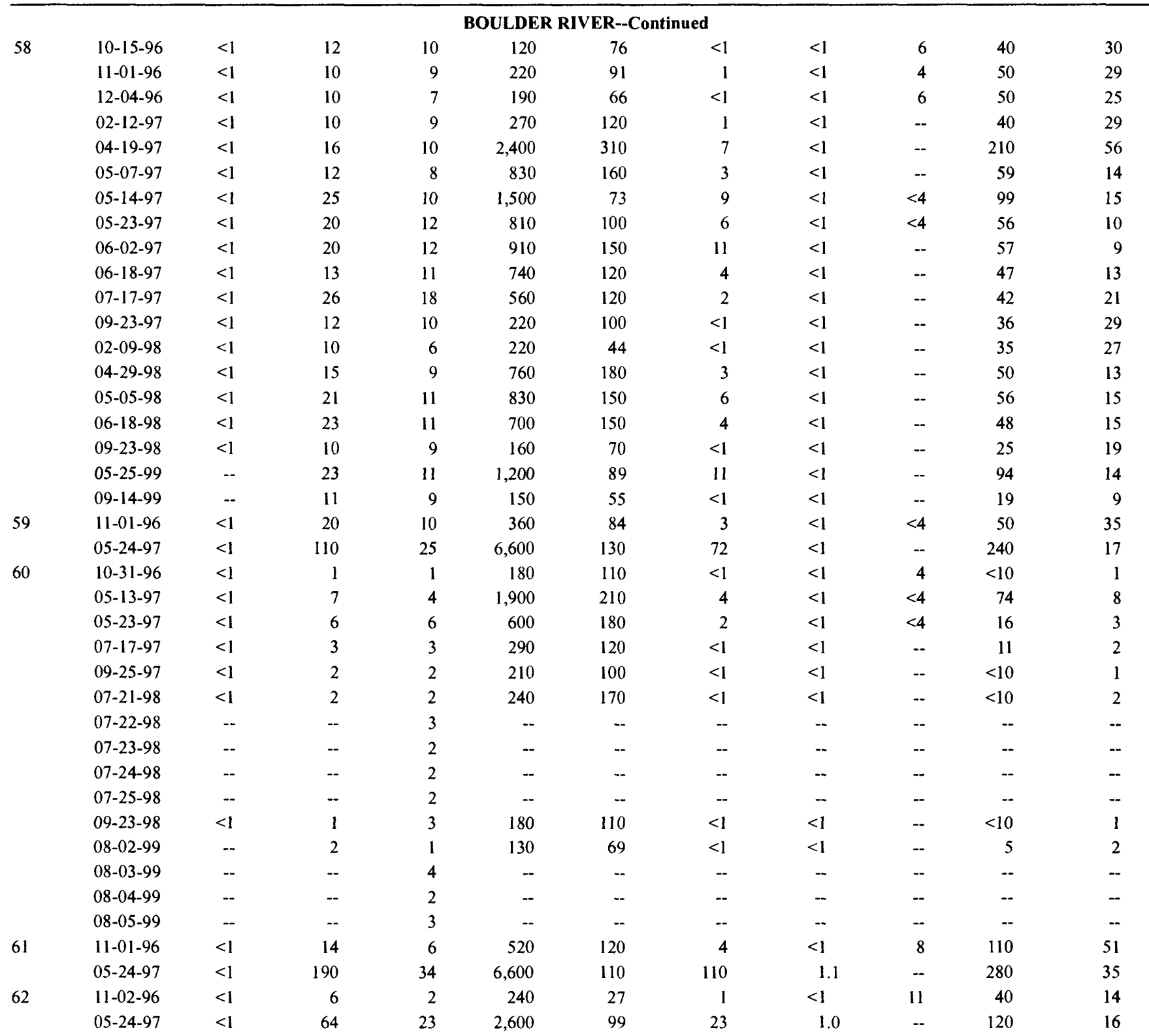


Table 2. Water-quality data for streams in the Boulder River watershed, Montana, 1996-99 (Continued)

\begin{tabular}{|c|c|c|c|c|c|c|c|c|c|c|c|}
\hline $\begin{array}{c}\text { Site } \\
\text { num- } \\
\text { ber } \\
\text { (fig. 2) }\end{array}$ & Date & $\begin{array}{c}\text { Mercury, } \\
\text { total } \\
\text { recov- } \\
\text { erable } \\
(\mu \mathrm{g} / \mathrm{L} \text { as } \\
\mathrm{Hg})\end{array}$ & $\begin{array}{c}\text { Mercury, } \\
\text { dis- } \\
\text { solved } \\
(\mu \mathrm{g} / \mathrm{L} \text { as } \\
\mathrm{Hg})\end{array}$ & $\begin{array}{c}\text { Molyb- } \\
\text { denum, } \\
\text { dis- } \\
\text { solved } \\
(\mu \mathrm{g} / \mathrm{L} \text { as } \\
\text { Mo) }\end{array}$ & $\begin{array}{c}\text { Nickel, } \\
\text { total } \\
\text { recov- } \\
\text { erable } \\
(\mu \mathrm{g} / \mathrm{L} \text { as } \\
\mathrm{Ni})\end{array}$ & $\begin{array}{l}\text { Nickel, } \\
\text { dis- } \\
\text { solved } \\
(\mu \mathrm{g} / \mathrm{L} \text { as } \\
\mathrm{Ni})\end{array}$ & $\begin{array}{l}\text { Silver, } \\
\text { total } \\
\text { recov- } \\
\text { erable } \\
(\mu \mathrm{g} / \mathrm{L} \text { as } \\
\mathrm{Ag})\end{array}$ & $\begin{array}{l}\text { Silver, } \\
\text { dis- } \\
\text { solved } \\
(\mu \mathrm{g} / \mathrm{L} \text { as } \\
\mathrm{Ag})\end{array}$ & $\begin{array}{c}\text { Uranium, } \\
\text { dis- } \\
\text { solved } \\
(\mu \mathrm{g} / \mathrm{L} \text { as } \\
\mathrm{U})\end{array}$ & $\begin{array}{c}\text { Zinc, } \\
\text { total } \\
\text { recov- } \\
\text { erable } \\
(\mu \mathrm{g} / \mathrm{L} \text { as } \\
\mathrm{Zn})\end{array}$ & $\begin{array}{c}\text { Zinc, } \\
\text { dis- } \\
\text { solved } \\
(\mu \mathrm{g} / \mathrm{L} \text { as } \\
\mathrm{Zn})\end{array}$ \\
\hline
\end{tabular}

\begin{tabular}{|c|c|c|c|c|c|c|c|c|c|c|c|}
\hline \multicolumn{12}{|c|}{ BOULDER RIVER-Continued } \\
\hline \multirow[t]{19}{*}{58} & $10-15-96$ & $<.1$ & $<.1$ & 1 & $<1$ & $<1$ & $<1$ & $<1$ & 2 & 210 & 189 \\
\hline & $11-01-96$ & $<.1$ & -- & 1 & -- & $<1$ & - & $<1$ & 2 & 230 & 208 \\
\hline & $12-04-96$ & $<.1$ & $<.1$ & $<1$ & $<1$ & $<1$ & $<1$ & $<1$ & 2 & 210 & 179 \\
\hline & $02-12-97$ & -- & -- & $<1$ & - & 1 & -- & $<1$ & 2 & 210 & 194 \\
\hline & $04-19-97$ & -- & -- & $<1$ & -- & $<1$ & -- & $<1$ & $<1$ & 140 & 65 \\
\hline & $05-07-97$ & -- & -- & $<1$ & - & $<1$ & -- & $<1$ & $<1$ & 70 & 44 \\
\hline & $05-14-97$ & $<.1$ & -- & $<1$ & 1 & $<1$ & $<1$ & $<1$ & $<1$ & 100 & 49 \\
\hline & $05-23-97$ & $<.1$ & -- & $<1$ & 1 & $<1$ & $<1$ & $<1$ & $<1$ & 80 & 47 \\
\hline & $06-02-97$ & -- & -- & $<1$ & -- & $<1$ & -- & $<1$ & $<1$ & 70 & 40 \\
\hline & $06-18-97$ & -- & -- & $<1$ & -- & $<1$ & -- & $<1$ & $<1$ & 60 & 37 \\
\hline & $07-17-97$ & - & -- & $<1$ & -- & $<1$ & -- & $<1$ & $<1$ & 110 & 73 \\
\hline & $09-23-97$ & -- & -- & 1 & -- & $<1$ & -- & $<1$ & 2 & 140 & 115 \\
\hline & $02-09-98$ & -- & -- & $<1$ & -- & $<1$ & -- & $<1$ & 2 & 150 & 140 \\
\hline & $04-29-98$ & -- & -- & $<1$ & -- & $<1$ & -- & $<1$ & $<1$ & 70 & 46 \\
\hline & $05-05-98$ & -- & -- & $<1$ & -- & $<1$ & -- & $<1$ & $<1$ & 70 & 43 \\
\hline & $06-18-98$ & -- & -- & $<1$ & -- & $<1$ & -- & $<1$ & $<1$ & 70 & 51 \\
\hline & $09-23-98$ & -- & -- & 1 & -- & $<1$ & -- & $<1$ & 2 & 110 & 100 \\
\hline & $05-25-99$ & -- & -- & - & -- & - & -- & -- & -- & 86 & 40 \\
\hline & $09-14-99$ & -- & -- & -- & -- & -- & -- & - & -- & 130 & 117 \\
\hline \multirow[t]{2}{*}{59} & $11-01-96$ & $<.1$ & -- & 2 & -- & $<1$ & -- & $<1$ & 3 & 210 & 189 \\
\hline & $05-24-97$ & $<.1$ & -- & $<1$ & -- & $<1$ & -- & $<1$ & $<1$ & 250 & 89 \\
\hline \multirow[t]{15}{*}{60} & $10-31-96$ & $<.1$ & $<.1$ & 3 & $<1$ & $<1$ & $<1$ & $<1$ & 3 & $<10$ & 1 \\
\hline & $05-13-97$ & $<.1$ & -- & $<1$ & $<1$ & $<1$ & $<1$ & $<1$ & $<1$ & 10 & 3 \\
\hline & $05-23-97$ & $<.1$ & -- & $<1$ & $<1$ & $<1$ & $<1$ & $<1$ & $<1$ & $<10$ & 4 \\
\hline & $07-17-97$ & -- & -- & 2 & -- & $<1$ & -- & $<1$ & 2 & $<10$ & 2 \\
\hline & $09-25-97$ & -- & -- & 2 & -- & $<1$ & - & $<1$ & 2 & $<10$ & 2 \\
\hline & $07-21-98$ & - & -- & 2 & -- & $<1$ & -- & $<1$ & 2 & $<10$ & 2 \\
\hline & $07-22-98$ & - & -- & -- & -- & -- & -- & -- & -- & -- & $<20$ \\
\hline & $07-23-98$ & -- & - & -- & -- & -- & -- & -- & -- & -- & $<20$ \\
\hline & $07-24-98$ & - & -. & -- & -- & -- & -- & -- & -- & -- & $<20$ \\
\hline & $07-25-98$ & - & -- & -- & -- & -- & -- & -- & - & -- & $<20$ \\
\hline & $09-23-98$ & -- & -- & 3 & -- & $<1$ & -- & $<1$ & 2 & $<10$ & 3 \\
\hline & 08-02-99 & -- & -- & -- & -- & -- & -- & -- & -- & $<40$ & 2 \\
\hline & $08-03-99$ & -- & -- & -- & -- & -- & -- & -- & -- & -- & 3 \\
\hline & 08-04-99 & -- & -- & -- & -- & -- & -- & -- & -- & -- & 2 \\
\hline & $08-05-99$ & -- & -- & -- & -- & -- & -- & -- & -- & -- & 5 \\
\hline \multirow[t]{2}{*}{61} & $11-01-96$ & $<.1$ & -- & 3 & -- & $<1$ & - & $<1$ & 3 & 180 & 138 \\
\hline & $05-24-97$ & $<.1$ & -. & $<1$ & -- & $<1$ & -- & $<1$ & $<1$ & 480 & 154 \\
\hline \multirow[t]{2}{*}{62} & $11-02-96$ & $<.1$ & -- & 2 & -- & $<1$ & -- & $<1$ & 4 & 30 & 14 \\
\hline & $05-24-97$ & $<.1$ & -- & 1 & -- & $<1$ & -- & $<1$ & 2 & 190 & 57 \\
\hline
\end{tabular}


Table 2. Water-quality data for streams in the Boulder River watershed, Montana, 1996-99 (Continued)

\begin{tabular}{|c|c|c|c|c|c|c|c|c|c|c|}
\hline $\begin{array}{c}\text { Site } \\
\text { num- } \\
\text { ber } \\
\text { (fig. 2) }\end{array}$ & Station name & Date & Time & $\begin{array}{c}\text { Dis- } \\
\text { charge, } \\
\text { instanta- } \\
\text { neous } \\
\left(\mathrm{ft}^{3} / \mathrm{s}\right)\end{array}$ & $\begin{array}{c}\text { Sedi- } \\
\text { ment, } \\
\text { sus- } \\
\text { pended } \\
\text { (mg/L) }\end{array}$ & $\begin{array}{c}\text { Sedi- } \\
\text { ment, } \\
\text { sus- } \\
\text { pended, } \\
\text { diameter } \\
\text { (percent } \\
\text { finer } \\
\text { than } \\
\mathbf{0 . 0 6 2} \\
\text { mm) }\end{array}$ & $\begin{array}{l}\text { Spe- } \\
\text { cific } \\
\text { con- } \\
\text { duct- } \\
\text { ance, } \\
\text { field } \\
(\mu \mathrm{S} / \mathrm{cm})\end{array}$ & $\begin{array}{c}\text { pH, } \\
\text { field } \\
\text { (stan- } \\
\text { dard } \\
\text { units) }\end{array}$ & $\begin{array}{l}\text { Tem- } \\
\text { pera- } \\
\text { ture, } \\
\text { water } \\
\left({ }^{\circ} \mathrm{C}\right)\end{array}$ & $\begin{array}{c}\text { Hard- } \\
\text { ness } \\
(\mathrm{mg} / \mathrm{L} \\
\text { as } \\
\left.\mathrm{CaCO}_{3}\right)\end{array}$ \\
\hline \multicolumn{11}{|c|}{ BASIN CREEK } \\
\hline \multirow[t]{5}{*}{5} & Basin Creek above Buckeye Mine, near Basin & $10-16-96$ & 1030 & -- & - & -- & 78 & - & -- & -- \\
\hline & & $09-25-98$ & 1345 & .44 & 29 & 99 & 79 & 7.6 & 5.0 & 32 \\
\hline & & $10-16-98$ & 1045 & .43 & 1 & 80 & 76 & 7.8 & 1.0 & 31 \\
\hline & & $05-28-99$ & 1010 & 7.9 & -- & - & 37 & 8.1 & 2.0 & 16 \\
\hline & & $09-13-99$ & 1115 & .34 & 15 & 29 & 79 & 7.8 & 5.0 & 33 \\
\hline \multirow[t]{3}{*}{6} & Buckeye Mine tributary near Basin & $10-16-98$ & 1055 & .05 & 1 & 67 & 87 & 8.3 & 1.0 & 36 \\
\hline & & $05-28-99$ & 1030 & 1.7 & -- & -- & 42 & 7.1 & 6.0 & 16 \\
\hline & & $09-13-99$ & 1030 & .009 & 7 & 90 & 104 & 7.9 & 4.5 & 47 \\
\hline \multirow[t]{6}{*}{7} & Basin Creek below Buckeye Mine tributary, near & $05-27-97$ & 1130 & 12 & -- & -- & 42 & -- & 4.0 & - \\
\hline & Basin & $04-30-98$ & 1100 & 5.0 & 8 & 66 & 55 & 7.0 & 0.0 & 23 \\
\hline & & $05-07-98$ & 1315 & 10 & 48 & 46 & 45 & 7.7 & - & 18 \\
\hline & & $10-16-98$ & 1340 & .48 & 1 & 75 & 75 & 7.7 & .5 & 31 \\
\hline & & $05-28-99$ & 0945 & 9.2 & -- & -- & 39 & 7.2 & 2.5 & 16 \\
\hline & & $09-13-99$ & 1130 & .37 & 2 & 64 & 80 & 7.8 & 5.0 & 33 \\
\hline \multirow[t]{8}{*}{8} & Basin Creek below Buckeye Mine, near Basin & $10-16-96$ & 1010 & .51 & 1 & 62 & 82 & 7.3 & 0.0 & 34 \\
\hline & & $05-27-97$ & 1045 & 15 & 10 & 36 & 44 & 7.1 & 5.0 & 16 \\
\hline & & $04-30-98$ & 1015 & 6.3 & 16 & 32 & 57 & 7.3 & 3.0 & 23 \\
\hline & & $05-07-98$ & 1445 & 17 & 74 & 77 & 49 & 7.0 & 3.0 & 17 \\
\hline & & $09-25-98$ & 1215 & .49 & 4 & 79 & 80 & 7.8 & 8.0 & 32 \\
\hline & & $10-16-98$ & 1315 & .66 & 4 & 82 & 75 & 7.6 & .5 & 27 \\
\hline & & $05-28-99$ & 0840 & 13 & -- & -- & 39 & 6.7 & 2.0 & 16 \\
\hline & & $09-13-99$ & 1250 & .35 & 1 & 82 & 82 & 7.9 & 11.0 & 33 \\
\hline \multirow[t]{2}{*}{9} & Grub Gulch near Basin & $10-16-96$ & 1115 & - & -- & - & 73 & -- & 0.0 & -- \\
\hline & & $05-27-97$ & 1300 & 13 & -- & -- & 36 & -- & 5.0 & -- \\
\hline \multirow[t]{2}{*}{10} & Clear Creek near Basin & $10-30-96$ & 1315 & .08 & -- & - & 122 & -- & 0.0 & .. \\
\hline & & $05-27-97$ & 1330 & 11 & -- & -- & 43 & -- & 4.5 & -- \\
\hline \multirow[t]{2}{*}{11} & Jimmys Creek near Basin & $10-30-96$ & 1330 & .02 & - & -- & 41 & -- & 4.5 & -- \\
\hline & & $05-27-97$ & 1345 & 5.5 & -- & -- & 16 & -. & 6.0 & -- \\
\hline \multirow[t]{2}{*}{12} & Basin Creek above Joe Bowers Creek, near Basin & $10-16-96$ & 1145 & -- & -- & - & 90 & -- & 0.0 & -- \\
\hline & & $05-27-97$ & 1505 & 75 & -- & -- & 29 & - & 6.0 &.- \\
\hline \multirow[t]{2}{*}{13} & Joe Bowers Creek near Basin & $10-30-96$ & 1250 & .32 & -- & -- & 44 & -- & 0.0 & -- \\
\hline & & $05-27-97$ & 1430 & 10 & -- & -- & 18 & -- & 6.5 & -- \\
\hline 14 & Basin Creek at Joe Bowers Creek, near Basin & $05-27-97$ & 1500 & 85 & -- & - & 27 & - & 6.5 & - \\
\hline \multirow[t]{2}{*}{15} & Weasel Gulch near Basin & $10-30-96$ & 1400 & .10 & -- & -- & 39 & -- & 4.0 & -- \\
\hline & & $05-27-97$ & 1545 & 5.9 & -- & -- & 17 & -- & 5.0 & -- \\
\hline
\end{tabular}


Table 2. Water-quality data for streams in the Boulder River watershed, Montana, 1996-99 (Continued)

\begin{tabular}{|c|c|c|c|c|c|c|c|c|c|c|c|}
\hline $\begin{array}{c}\text { Site } \\
\text { num- } \\
\text { ber } \\
\text { (fig. 2) }\end{array}$ & Date & $\begin{array}{c}\text { Cal- } \\
\text { cium, } \\
\text { dissolved } \\
(\mathbf{m g} / \mathbf{L} \\
\text { as } \mathrm{Ca})\end{array}$ & $\begin{array}{l}\text { Magne- } \\
\text { sium, } \\
\text { dissolved } \\
\text { (mg/L } \\
\text { as } \mathbf{M g )}\end{array}$ & $\begin{array}{c}\text { Sodium, } \\
\text { dissolved } \\
\text { (mg/L } \\
\text { as } \mathbf{N a )}\end{array}$ & $\begin{array}{l}\text { Potas- } \\
\text { sium, } \\
\text { dissolved } \\
\text { (mg/L } \\
\text { as } \mathbf{K})\end{array}$ & $\begin{array}{c}\mathrm{ANC}, \\
\text { lab } \\
(\mathrm{mg} / \mathrm{L} \\
\left.\text { as } \mathrm{CaCO}_{3}\right)\end{array}$ & $\begin{array}{c}\text { Sulfate, } \\
\text { dissolved } \\
\text { (mg/L } \\
\left.\text { as } \mathrm{SO}_{4}\right)\end{array}$ & $\begin{array}{c}\text { Chloride, } \\
\text { dissolved } \\
\text { (mg/L } \\
\text { as } \mathrm{Cl} \text { ) }\end{array}$ & $\begin{array}{c}\text { Fluoride, } \\
\text { dissolved } \\
\text { (mg/L } \\
\text { as F) }\end{array}$ & $\begin{array}{c}\text { Silica, } \\
\text { dissolved } \\
(\mathrm{mg} / \mathrm{L} \\
\left.\text { as } \mathrm{SiO}_{2}\right)\end{array}$ & $\begin{array}{c}\text { Dis- } \\
\text { solved } \\
\text { solids, } \\
\text { calcu- } \\
\text { lated } \\
(\mathrm{mg} / \mathrm{L})\end{array}$ \\
\hline
\end{tabular}

\begin{tabular}{|c|c|c|c|c|c|c|c|c|c|c|c|}
\hline \multirow[b]{2}{*}{5} & \multicolumn{11}{|c|}{ BASIN CREEK } \\
\hline & $10-16-96$ & -- & - & -- & - & - & -- & - & - & -- & - \\
\hline & $09-25-98$ & 9.5 & 2.1 & 2.2 & 1.1 & 32 & 6.8 & .19 & $<.10$ & 14 & 55 \\
\hline & $10-16-98$ & 9.0 & 2.1 & 2.0 & 1.1 & 31 & 6.7 & .24 & $<.10$ & 14 & 53 \\
\hline & $05-28-99$ & 4.8 & 1.0 & 1.1 & .68 & 15 & 2.7 & .24 & -- & -- & -- \\
\hline & $09-13-99$ & 9.5 & 2.3 & 2.1 & 1.1 & 33 & 6.7 & $<.29$ & - & - & -- \\
\hline \multirow[t]{3}{*}{6} & $10-16-98$ & 10 & 2.6 & 2.5 & 1.0 & 35 & 7.1 & .26 & $<.10$ & 11 & 55 \\
\hline & $05-28-99$ & 4.8 & 1.0 & 1.3 & .64 & 11 & 6.6 & .84 & -- & -- & -- \\
\hline & $09-13-99$ & 13 & 3.2 & 2.7 & .99 & 47 & 5.0 & $<.29$ & -- & -- & -- \\
\hline \multirow[t]{6}{*}{7} & $05-27-97$ & - & -- & - & - & -- & -- & - & -- & -- & - \\
\hline & $04-30-98$ & 6.7 & 1.5 & 1.3 & 1.0 & 19 & 6.5 & .23 & $<.10$ & 8.9 & 38 \\
\hline & $05-07-98$ & 5.4 & 1.2 & 1.1 & .77 & 14 & 5.8 & .27 & $<.10$ & 7.2 & 31 \\
\hline & $10-16-98$ & 9.0 & 2.1 & 2.1 & .94 & 31 & 6.8 & .21 & $<.10$ & 13 & 53 \\
\hline & $05-28-99$ & 4.8 & 1.0 & 1.2 & .67 & 14 & 3.5 & .21 & -. & .. & -- \\
\hline & $09-13-99$ & 9.5 & 2.3 & 2.1 & 1.1 & 34 & 6.7 & $<.29$ & -- & -- & - \\
\hline \multirow[t]{8}{*}{8} & $10-16-96$ & 10 & 2.3 & 2.3 & 1.0 & 32 & 8.7 & .30 & $<.10$ & 13 & 57 \\
\hline & $05-27-97$ & 4.8 & 1.1 & 1.2 & .67 & 12 & 7.6 & .19 & $<.10$ & 9.6 & 33 \\
\hline & $04-30-98$ & 6.8 & 1.5 & 1.3 & 1.2 & 20 & 7.1 & .28 & $<.10$ & 8.9 & 40 \\
\hline & $05-07-98$ & 5.0 & 1.1 & 1.1 & .91 & 10 & 8.7 & .22 & $<.10$ & 7.8 & 32 \\
\hline & $09-25-98$ & 9.3 & 2.2 & 2.2 & .99 & 31 & 7.8 & .19 & $<.10$ & 14 & 56 \\
\hline & $10-16-98$ & 7.7 & 1.8 & 2.0 & .83 & 29 & 7.5 & .22 & $<.10$ & 13 & 51 \\
\hline & $05-28-99$ & 4.7 & 1.0 & 1.2 & .68 & 13 & 4.7 & .22 & -- & -- & -- \\
\hline & $09-13-99$ & 9.5 & 2.3 & 2.3 & 1.1 & 32 & 8.0 & $<.29$ & - & - & -- \\
\hline \multirow[t]{2}{*}{9} & $10-16-96$ & -- & -- & - & - & - & -- & $\cdots$ & -- & -- & - \\
\hline & $05-27-97$ & -- & -- & -- & -- & - & -- & -- & - & -- & - \\
\hline \multirow[t]{2}{*}{10} & $10-30-96$ & -- & - & -- & -- & -- & -- & -- & -- & -- & - \\
\hline & $05-27-97$ & - & -- & -- & -- & -- & -- & -- & -- & -- & - \\
\hline \multirow[t]{2}{*}{11} & $10-30-96$ & - & -- & -- & -- & - & -- & - & -- & -- & -. \\
\hline & $05-27-97$ & -- & -- & -- & -. & - & -- & -- & -. & -- & -. \\
\hline \multirow[t]{2}{*}{12} & $10-16-96$ & -- & -- & -- & -- & -- & -- & -- & - & -. & -. \\
\hline & $05-27-97$ & - & -- & -- & -- & -- & -- & -- & - & -- & - \\
\hline \multirow[t]{2}{*}{13} & $10-30-96$ & - & - & -- & - & -- & -- & - & -- & - & - \\
\hline & $05-27-97$ & -- & -- & - & -- & -- & -- & -- & - & - & - \\
\hline 14 & $05-27-97$ & -- & - & -- & - & -. & -- & -- & - & - & - \\
\hline \multirow[t]{2}{*}{15} & $10-30-96$ & -- & -- & -- & -- & -- & -- & - & -- & - & -- \\
\hline & $05-27-97$ & -- & -- & -- & -- & -- & -- & - & -- & -- & -- \\
\hline
\end{tabular}


Table 2. Water-quality data for streams in the Boulder River watershed, Montana, 1996-99 (Continued)

\begin{tabular}{|c|c|c|c|c|c|c|c|}
\hline $\begin{array}{c}\text { Site } \\
\text { num- } \\
\text { ber } \\
\text { (fig. 2) }\end{array}$ & Date & $\begin{array}{c}\text { Nitrite, } \\
\text { dissolved } \\
\text { (mg/L } \\
\text { as } \mathbf{N})\end{array}$ & $\begin{array}{c}\text { Nitrite } \\
\text { plus } \\
\text { nitrate, } \\
\text { dissolved } \\
\text { (mg/L } \\
\text { as } \mathbf{N})\end{array}$ & $\begin{array}{c}\text { Ammonia, } \\
\text { dissolved } \\
\text { (mg/L } \\
\text { as } \mathrm{N} \text { ) }\end{array}$ & $\begin{array}{c}\text { Ammonia plus } \\
\text { organic } \\
\text { nitrogen, } \\
\text { total } \\
\text { (mg/L } \\
\text { as } \mathbf{N})\end{array}$ & $\begin{array}{c}\text { Phos- } \\
\text { phorus, } \\
\text { total } \\
\text { (mg/L } \\
\text { as P) }\end{array}$ & $\begin{array}{c}\text { Phos- } \\
\text { phorus, } \\
\text { ortho- } \\
\text { phosphate, } \\
\text { dissolved } \\
\text { (mg/L } \\
\text { as P) }\end{array}$ \\
\hline
\end{tabular}

\begin{tabular}{|c|c|c|c|c|c|c|c|}
\hline \multicolumn{8}{|c|}{ BASIN CREEK } \\
\hline \multirow[t]{5}{*}{5} & $10-16-96$ & -- & -- & -- & -- & -- & -- \\
\hline & $09-25-98$ & -- & -- & -- & $<.1$ & .011 & -- \\
\hline & $10-16-98$ & -- & - & - & -- & -- & - \\
\hline & $05-28-99$ & -- & -- & -- & - & -- & - \\
\hline & $09-13-99$ & - & - & -- & - & -- & - \\
\hline \multirow[t]{3}{*}{6} & $10-16-98$ & -- & - & -- & - & -. & - \\
\hline & $05-28-99$ & -- & -- & -- & -- & -- & - \\
\hline & $09-13-99$ & - & -- & -- & -- & -- & -- \\
\hline \multirow[t]{6}{*}{7} & $05-27-97$ & -- & -- & -- & -- & -- & -- \\
\hline & $04-30-98$ & -- & -- & -- & -- & -- & -- \\
\hline & $05-07-98$ & - & -- & -- & -- & -- & -- \\
\hline & $10-16-98$ & -- & -- & -- & -- & -- & -- \\
\hline & $05-28-99$ & - & -- & -- & -- & -- & -. \\
\hline & $09-13-99$ & -- & -- & -. & -- & -- & -. \\
\hline \multirow[t]{8}{*}{8} & $10-16-96$ & -- & -- & -- & - & -- & -- \\
\hline & $05-27-97$ & -- & -- & -- & -- & -- & -- \\
\hline & $04-30-98$ & -- & -- & -- & - &.- & -- \\
\hline & $05-07-98$ & -- & -- & .. & - & -. & -. \\
\hline & $09-25-98$ & -- & -- & -- & $<.1$ & $<.05$ & - \\
\hline & $10-16-98$ & - & - & -. & -- & -. & -- \\
\hline & $05-28-99$ & - & -- & -. & -- & -. & -- \\
\hline & $09-13-99$ & -- & -- & -- & -- & -- & -- \\
\hline \multirow[t]{2}{*}{9} & $10-16-96$ & -- & -- & -- & -- & -. & -. \\
\hline & $05-27-97$ & -- & -- & -- & -- & -. & -- \\
\hline \multirow[t]{2}{*}{10} & $10-30-96$ & - & - & .. & -. & -- & -- \\
\hline & $05-27-97$ & -- & -- & -- & -- & -- & -- \\
\hline \multirow[t]{2}{*}{11} & $10-30-96$ & -- & -- & -- & -- & -- & - \\
\hline & $05-27-97$ & -- & -- & -- & - & -- & -. \\
\hline \multirow[t]{2}{*}{12} & $10-16-96$ & -- & -- & -- & - & -. & - \\
\hline & $05-27-97$ & -- & -- & -- & -- & -- & - \\
\hline \multirow[t]{2}{*}{13} & $10-30-96$ & -- & -- & -- & -- & -- & -- \\
\hline & $05-27-97$ & -- & -- & - & - & -- & -- \\
\hline 14 & $05-27-97$ & -- & -- & -- & -- & -- & -- \\
\hline \multirow[t]{2}{*}{15} & $10-30-96$ & -- & -- & -- & -- & -- & -- \\
\hline & $05-27-97$ & $-\cdot$ & - & - & -- & -- & - \\
\hline
\end{tabular}


Table 2. Water-quality data for streams in the Boulder River watershed, Montana, 1996-99 (Continued)

\begin{tabular}{|c|c|c|c|c|c|c|c|c|c|c|c|c|}
\hline $\begin{array}{c}\text { Site } \\
\text { num- } \\
\text { ber } \\
\text { (fig. 2) }\end{array}$ & Date & $\begin{array}{c}\text { Alumi- } \\
\text { num, } \\
\text { total } \\
\text { recov- } \\
\text { erable } \\
(\mu \mathrm{g} / \mathrm{L} \text { as } \\
\text { Al) }\end{array}$ & $\begin{array}{l}\text { Alumi- } \\
\text { num, } \\
\text { dis- } \\
\text { solved } \\
(\mu \mathrm{g} / \mathrm{L} \text { as } \\
\text { Al) }\end{array}$ & $\begin{array}{l}\text { Anti- } \\
\text { mony, } \\
\text { dis- } \\
\text { solved } \\
(\mu \mathrm{g} / \mathrm{L} \text { as } \\
\text { Sb) }\end{array}$ & $\begin{array}{c}\text { Arsenic, } \\
\text { total } \\
\text { recov- } \\
\text { erable } \\
(\mu \mathrm{g} / \mathrm{L} \text { as } \\
\text { As) }\end{array}$ & $\begin{array}{l}\text { Arsenic, } \\
\text { dis- } \\
\text { solved } \\
(\mu \mathrm{g} / \mathrm{L} \text { as } \\
\text { As) }\end{array}$ & $\begin{array}{c}\text { Barium, } \\
\text { dis- } \\
\text { solved } \\
(\mu \mathrm{g} / \mathbf{L} \text { as } \\
\text { Ba })\end{array}$ & $\begin{array}{l}\text { Beryl- } \\
\text { lium, } \\
\text { dis- } \\
\text { solved } \\
(\mu \mathrm{g} / \mathrm{L} \text { as } \\
\text { Be })\end{array}$ & $\begin{array}{l}\text { Cad- } \\
\text { mium, } \\
\text { total } \\
\text { recov- } \\
\text { erable } \\
(\mu \mathrm{g} / \mathrm{L} \text { as } \\
\text { Cd })\end{array}$ & $\begin{array}{c}\text { Cad- } \\
\text { mium, } \\
\text { dis- } \\
\text { solved } \\
(\mu \mathrm{g} / \mathrm{L} \text { as } \\
\text { Cd })\end{array}$ & $\begin{array}{c}\text { Chro- } \\
\text { mium, } \\
\text { total } \\
\text { recov- } \\
\text { erable } \\
(\mu \mathrm{g} / \mathrm{L} \text { as } \\
\text { Cr })\end{array}$ & $\begin{array}{c}\text { Chro- } \\
\text { mium, } \\
\text { dis- } \\
\text { solved } \\
(\mu \mathrm{g} / \mathrm{L} \mathrm{a} \\
\mathrm{Cr})\end{array}$ \\
\hline \multicolumn{13}{|c|}{ BASIN CREEK } \\
\hline \multirow[t]{4}{*}{5} & $10-16-96$ & -- & -- & -- & -- & - & -- & -- & - & -- & - & -- \\
\hline & $09-25-98$ & 1,300 & 8.3 & $<1$ & 4 & 3 & 8.1 & $<1$ & $<1$ & $<.3$ & -- & $<1$ \\
\hline & $10-16-98$ & 17 & 2.3 & $<1$ & 2 & 1 & 4.7 & $<1$ & $<1$ & $<.3$ & -- & $<1$ \\
\hline & $05-28-99$ & 185 & 52 & -- & 4 & 2 & -- & -- & $<1$ & $<.3$ & - & - \\
\hline \multirow[t]{6}{*}{7} & $05-27-97$ & - & -- & - & -- & -- & -- & -- & -- & -- & -- & -- \\
\hline & $04-30-98$ & 350 & 149 & $<1$ & 5 & 3 & 6.1 & $<1$ & $<1$ & $<.3$ & -- & $<1$ \\
\hline & $05-07-98$ & 900 & 102 & $<1$ & 10 & 7 & 5.5 & $<1$ & $<1$ & .5 & - & $<1$ \\
\hline & $10-16-98$ & $<10$ & 13 & $<1$ & 2 & 2 & 4.7 & $<1$ & $<1$ & $<.3$ & -- & $<1$ \\
\hline & $05-28-99$ & 220 & 66 & -- & 3 & 2 & -- & -- & $<1$ & .4 & -- & - \\
\hline & $09-13-99$ & 41 & 5 & - & 3 & 3 & -- & -- & $<1$ & $<.3$ & -- & - \\
\hline \multirow[t]{5}{*}{8} & $10-16-96$ & -- & 22 & 3 & 19 & 16 & 5.0 & $<1$ & $<1$ & .2 & $<1$ & $<1$ \\
\hline & $05-27-97$ & 430 & 225 & 2 & 36 & 13 & 6.1 & $<1$ & 1 & 1.2 & $<1$ & $<1$ \\
\hline & $04-30-98$ & 430 & 154 & 3 & 52 & 29 & 7.9 & $<1$ & $<1$ & .8 & -- & $<1$ \\
\hline & $05-07-98$ & 1,300 & 219 & 7 & 500 & 38 & 8.3 & $<1$ & 2 & 1.4 & - & $<1$ \\
\hline & $09-25-98$ & 90 & 13 & 4 & 24 & 21 & 5.8 & $<1$ & $<1$ & $<.3$ & -- & $<1$ \\
\hline \multirow[t]{2}{*}{11} & $10-30-96$ & -- & -- & - & -- & -- & - & - & -- & -- & -- & -- \\
\hline & $05-27-97$ & -- & - & -- & -- & -- & -- & - & -- & -- & - & - \\
\hline \multirow[t]{2}{*}{12} & $10-16-96$ & - & -- & -- & -- & -- & -- & -- & -- & -- & -- & - \\
\hline & $05-27-97$ & - & -- & - & -- & -- & -- & -- & -- & -- & -- & -- \\
\hline \multirow[t]{2}{*}{13} & $10-30-96$ & -- & -- & - & -- & -- & -- & - & -- & -- & - & -- \\
\hline & $05-27-97$ & - & -- & -- & -- & -- & - & -- & - & -- & - & - \\
\hline 14 & $05-27-97$ & -- & -- & -- & -- & -- & -- & - & -- & -- & -- & -- \\
\hline \multirow[t]{2}{*}{15} & $10-30-96$ & -- & - & -- & -- & -- & -- & -- & -- & -- & -- & -- \\
\hline & $05-27-97$ & -- & - & -. & -- & -. & -- & - & -- & -- & -- & -- \\
\hline
\end{tabular}


Table 2. Water-quality data for streams in the Boulder River watershed, Montana, 1996-99 (Continued)

\begin{tabular}{|c|c|c|c|c|c|c|c|c|c|c|c|}
\hline $\begin{array}{c}\text { Site } \\
\text { num- } \\
\text { ber } \\
\text { (fig. 2) }\end{array}$ & Date & $\begin{array}{c}\text { Cobalt, } \\
\text { dis- } \\
\text { solved } \\
(\mu \mathrm{g} / \mathrm{L} \text { as } \\
\text { Co) }\end{array}$ & $\begin{array}{l}\text { Copper, } \\
\text { total recov- } \\
\text { erable } \\
(\mu \mathrm{g} / \mathrm{L} \text { as } \\
\text { Cu) }\end{array}$ & $\begin{array}{c}\text { Copper, } \\
\text { dis- } \\
\text { solved } \\
(\mu \mathrm{g} / \mathrm{L} \text { as } \\
\mathrm{Cu})\end{array}$ & $\begin{array}{c}\text { Iron, } \\
\text { total } \\
\text { recov- } \\
\text { erable } \\
(\mu \mathrm{g} / \mathrm{L} \text { as } \\
\text { Fe) }\end{array}$ & $\begin{array}{c}\begin{array}{c}\text { Iron, } \\
\text { dis- } \\
\text { solved }\end{array} \\
(\mu \mathrm{g} / \mathrm{L} \text { as } \\
\text { Fe })\end{array}$ & $\begin{array}{l}\text { Lead, } \\
\text { total } \\
\text { recov- } \\
\text { erable } \\
(\mu \mathrm{g} / \mathrm{L} \text { as } \\
\mathrm{Pb})\end{array}$ & $\begin{array}{c}\text { Lead, } \\
\text { dis- } \\
\text { solved } \\
(\mu \mathrm{g} / \mathrm{L} \text { as } \\
\text { Pb) }\end{array}$ & $\begin{array}{c}\text { Lithium, } \\
\text { dis- } \\
\text { solved } \\
(\mu \mathrm{g} / \mathrm{L} \text { as } \\
\mathrm{Li})\end{array}$ & $\begin{array}{c}\text { Manga- } \\
\text { nese, } \\
\text { total } \\
\text { recov- } \\
\text { erable } \\
(\mu \mathrm{g} / \mathbf{L} \text { as } \\
\mathbf{M n})\end{array}$ & $\begin{array}{c}\text { Manga- } \\
\text { nese, } \\
\text { dis- } \\
\text { solved } \\
(\mu \mathrm{g} / \mathrm{L} \text { as } \\
\text { Mn) }\end{array}$ \\
\hline
\end{tabular}

\begin{tabular}{|c|c|c|c|c|c|c|c|c|c|c|c|}
\hline \multirow[b]{2}{*}{5} & \multicolumn{11}{|c|}{ BASIN CREEK } \\
\hline & $10-16-96$ & -- & -- & - & -- & -- & - & -- & $-\cdot$ & -- & -- \\
\hline & $09-25-98$ & $<1$ & 3 & $<1$ & 1,200 & 17 & 7 & $<1$ & -- & 46 & 13 \\
\hline & $10-16-98$ & $<1$ & $<1$ & $<1$ & 71 & 48 & $<1$ & $<1$ & -- & $<10$ & 8 \\
\hline & $05-28-99$ & - & 2 & 2 & 240 & 61 & 1 & $<1$ & -- & 11 & 3 \\
\hline & $09-13-99$ & $-\cdots$ & $<1$ & $<1$ & 470 & 230 & $<1$ & $<1$ & - & 230 & 214 \\
\hline \multirow[t]{3}{*}{6} & $10-16-98$ & $<1$ & $<1$ & $<1$ & 190 & 50 & $<1$ & $<1$ & -- & 73 & 54 \\
\hline & $05-28-99$ & - & 14 & 11 & 460 & 79 & 1 & $<1$ & -- & 220 & 178 \\
\hline & $09-13-99$ & - & $<1$ & $<1$ & 75 & 60 & $<1$ & $<1$ & -- & 9 & 9 \\
\hline \multirow[t]{6}{*}{7} & $05-27-97$ & - & -- & -- & -- & -- & - & - & -- & -- & -- \\
\hline & $04-30-98$ & $<1$ & 9 & 9 & 600 & 190 & 1 & $<1$ & -- & 200 & 184 \\
\hline & $05-07-98$ & $<1$ & 7 & 5 & 2,000 & 160 & 7 & $<1$ & -- & 330 & 229 \\
\hline & $10-16-98$ & $<1$ & $<1$ & $<1$ & 130 & 103 & $<1$ & $<1$ & - & 26 & 28 \\
\hline & $05-28-99$ & $\cdots$ & 3 & 4 & 230 & 54 & $<1$ & $<1$ & -- & 43 & 39 \\
\hline & $09-13-99$ & -- & $<1$ & $<1$ & 340 & 130 & $<1$ & $<1$ & -- & 33 & 21 \\
\hline \multirow[t]{8}{*}{8} & $10-16-96$ & $<1$ & 4 & 2 & 170 & 130 & 3 & $<1$ & $<4$ & 70 & 65 \\
\hline & $05-27-97$ & $<1$ & 18 & 18 & 530 & 180 & 24 & 10 & $<4$ & 140 & 136 \\
\hline & $04-30-98$ & $<1$ & 12 & 11 & 880 & 240 & 28 & 7.7 & -- & 200 & 187 \\
\hline & $05-07-98$ & $<1$ & 40 & 18 & 3,200 & 240 & 340 & 23 & -- & 410 & 306 \\
\hline & $09-25-98$ & $<1$ & 3 & 2 & 250 & 93 & 2 & $<1$ & -- & 55 & 48 \\
\hline & $10-16-98$ & $<1$ & 3 & 2 & 380 & 88 & 9 & $<1$ & -- & 74 & 52 \\
\hline & $05-28-99$ & - & 11 & 9 & 350 & 100 & 17 & 5.4 & -- & 91 & 85 \\
\hline & 09-13-99 & - & 3 & 2 & 220 & 160 & 5 & 2.0 & -- & 61 & 55 \\
\hline \multirow[t]{2}{*}{9} & $10-16-96$ & - & -- & -. & -. & -- & -. & -- & -- & -. & -. \\
\hline & $05-27-97$ & - & -- & - & -- & -- & -- & -- & -- & -- & -- \\
\hline \multirow[t]{2}{*}{10} & $10-30-96$ & - & -- & $-\cdot$ & -- & -- & -- & -- & -- & -- & -- \\
\hline & $05-27-97$ & - & - & - & -- & -- & -- & -- & - & -- & -- \\
\hline \multirow[t]{2}{*}{11} & $10-30-96$ & - & - & - & -- & -- & -- & - & -- & - & -- \\
\hline & $05-27-97$ & - & - & -. & -- & - & -- & -- & -- & - & - \\
\hline \multirow[t]{2}{*}{12} & $10-16-96$ & - & -- & -. & - & -- & -- & -- & -- & -- & -- \\
\hline & $05-27-97$ & - & - & -- & -. & -- & -- &.- & -- & -- & -- \\
\hline \multirow[t]{2}{*}{13} & $10-30-96$ & -. & -- & - & -- & - &.- & - & - & -- & -- \\
\hline & $05-27-97$ & - & - & - & -- & -- & - & -- & - & -- & -- \\
\hline 14 & $05-27-97$ & -- & -. & - & -. & -- &.- & -- & - & - & -- \\
\hline \multirow[t]{2}{*}{15} & $10-30-96$ & -- & -- & -- & -- & -- & -- & -- & -- & -- & -- \\
\hline & $05-27-97$ & -- & - & - & -- & -- & -- & -- & -- & -- & -- \\
\hline
\end{tabular}


Table 2. Water-quality data for streams in the Boulder River watershed, Montana, 1996-99 (Continued)

\begin{tabular}{|c|c|c|c|c|c|c|c|c|c|c|c|}
\hline $\begin{array}{c}\text { Site } \\
\text { num- } \\
\text { ber } \\
\text { (fig. 2) }\end{array}$ & Date & $\begin{array}{c}\text { Mercury, } \\
\text { total } \\
\text { recov- } \\
\text { erable } \\
(\mu \mathrm{g} / \mathrm{L} \text { as } \\
\mathrm{Hg})\end{array}$ & $\begin{array}{c}\text { Mercury, } \\
\text { dis- } \\
\text { solved } \\
(\mu \mathrm{g} / \mathrm{L} \text { as } \\
\text { Hg) }\end{array}$ & $\begin{array}{c}\text { Molyb- } \\
\text { denum, } \\
\text { dis- } \\
\text { solved } \\
(\mu \mathrm{g} / \mathrm{L} \text { as } \\
\text { Mo })\end{array}$ & $\begin{array}{c}\text { Nickel, } \\
\text { total } \\
\text { recov- } \\
\text { erable } \\
(\mu \mathrm{g} / \mathrm{L} \text { as } \\
\mathrm{Ni})\end{array}$ & $\begin{array}{c}\text { Nickel, } \\
\text { dis- } \\
\text { solved } \\
(\mu \mathrm{g} / \mathrm{L} \text { as } \\
\text { Ni) }\end{array}$ & $\begin{array}{c}\text { Silver, } \\
\text { total } \\
\text { recov- } \\
\text { erable } \\
(\mu \mathrm{g} / \mathrm{L} \mathrm{as} \\
\mathrm{Ag})\end{array}$ & $\begin{array}{c}\text { Silver, } \\
\text { dis- } \\
\text { solved } \\
(\mu \mathrm{g} / \mathrm{L} \text { as } \\
\text { Ag) }\end{array}$ & $\begin{array}{c}\text { Uranium, } \\
\text { dis- } \\
\text { solved } \\
(\mu \mathrm{g} / \mathrm{L} \text { as } \\
\text { U) }\end{array}$ & $\begin{array}{c}\text { Zinc, } \\
\text { total } \\
\text { recov- } \\
\text { erable } \\
(\mu \mathrm{g} / \mathrm{L} \text { as } \\
\mathrm{Zn})\end{array}$ & $\begin{array}{c}\text { Zinc, } \\
\text { dis- } \\
\text { solved } \\
(\mu \mathrm{g} / \mathrm{L} \text { as } \\
\mathrm{Zn})\end{array}$ \\
\hline
\end{tabular}

\begin{tabular}{|c|c|c|c|c|c|c|c|c|c|c|c|}
\hline & & & & & & CRE & & & & & \\
\hline 5 & $10-16-96$ & - & .. & -- & -- & - & -- & - & -- & $<10$ & - \\
\hline & $09-25-98$ & -- & -. & $<1$ & -- & $<1$ & - & $<1$ & $<1$ & 20 & 3 \\
\hline & $10-16-98$ & -- & -. & $<1$ & - & $<1$ & -- & $<1$ & $<1$ & 10 & 7 \\
\hline & $05-28-99$ & -- & -- & -- & -- & -- & - & -. & -- & $<40$ & 6 \\
\hline & 09-13-99 & -- & -- & -- & -- & -- & -- & -- & -- & $<31$ & 7 \\
\hline 6 & $10-16-98$ & -- & -- & $<1$ & -- & $<1$ & - & $<1$ & $<1$ & 21 & 15 \\
\hline & $05-28-99$ & -- & -- &.- & -. & -- & -- & -- & - & 250 & 234 \\
\hline & $09-13-99$ & - & -- & -- & -- & -- & -- & -- & -. & e28 & 28 \\
\hline 7 & $05-27-97$ & - & -- & -- & -- & - & - & -- & -- & 100 & - \\
\hline & $04-30-98$ & -- & -. & $<1$ & -. & 1 & -. & $<1$ & $<1$ & 130 & 129 \\
\hline & $05-07-98$ & - & -- & $<1$ & -- & 1 & -- & $<1$ & $<1$ & 140 & 109 \\
\hline & $10-16-98$ & -- & -- & $<1$ & -- & $<1$ & -- & $<1$ & $<1$ & 15 & 12 \\
\hline & $05-28-99$ & - & -- & -- & $\ldots$ & -- & -- & -- & -- & 52 & 49 \\
\hline & $09-13-99$ & -- & -- & -- & -- & - & -. & -- & -- & $<31$ & 7 \\
\hline 8 & $10-16-96$ & $<.1$ & $<.1$ & $<1$ & $<1$ & $<1$ & $<1$ & $<1$ & $<1$ & 40 & 39 \\
\hline & $05-27-97$ & $<.1$ & -- & $<1$ & $<1$ & $<1$ & $<1$ & $<1$ & $<1$ & 180 & 173 \\
\hline & $04-30-98$ & -- & - & $<1$ & -- & 1 & -. & $<1$ & $<1$ & 150 & 140 \\
\hline & $05-07-98$ & -- &.- & $<1$ & -- & $<1$ & -- & $<1$ & $<1$ & 270 & 218 \\
\hline & $09-25-98$ & -- & - & $<1$ & - & $<1$ & - & $<1$ & $<1$ & 30 & 24 \\
\hline & $10-16-98$ & -- & -- & $<1$ & -- & $<1$ & - & $<1$ & $<1$ & 42 & 26 \\
\hline & $05-28-99$ & -- & - & -- & - & - & - & -- & -. & 95 & 90 \\
\hline & $09-13-99$ & -- & - & -- & - & -- & -- & - & - & 37 & 35 \\
\hline 9 & $10-16-96$ & -- & -- & -- & - & -- & -- & -- & -- & 20 & -- \\
\hline & $05-27-97$ & - & -- & - & - & - & -- & -- & - & 40 & - \\
\hline 10 & $10-30-96$ & -- & - & - & - & -- & - & -- & -- & 30 & -- \\
\hline & $05-27-97$ & -- & - & -- & -- & -- & -- & -- & -- & 30 & -- \\
\hline 11 & $10-30-96$ & - & - & -- & -- & -- & - & -- & -- & $<10$ & - \\
\hline & $05-27-97$ & -- & - & -- & - & -- & -. & -- & -- & $<10$ & -- \\
\hline 12 & $10-16-96$ & -- & -- & -- & -- & -- & -- & - & - & $<10$ & - \\
\hline & $05-27-97$ & -- & -- & -- & -- & -- & -- & -- & -- & 50 & - \\
\hline 13 & $10-30-96$ & -- & -- & -- &.- & -- & -- & -- & - & $<10$ & -- \\
\hline & $05-27-97$ & -- & -- & -- & -- & - & - & -- & -- & $<10$ & - \\
\hline 14 & $05-27-97$ & -- & - & -- & -- & -- & - & -- & -- & 40 & - \\
\hline 15 & $10-30-96$ & -- & -- & -- & -- & -- & -- & -- & -- & $<10$ & - \\
\hline & $05-27-97$ & -- & - & -- & -- & -- & -- & -- & -- & $<10$ & - \\
\hline
\end{tabular}


Table 2. Water-quality data for streams in the Boulder River watershed, Montana, 1996-99 (Continued)

\begin{tabular}{|c|c|c|c|c|c|c|c|c|c|c|}
\hline $\begin{array}{c}\text { Site } \\
\text { num- } \\
\text { ber } \\
\text { (fig. 2) }\end{array}$ & Station name & Date & Time & $\begin{array}{c}\begin{array}{c}\text { Dis- } \\
\text { charge, }\end{array} \\
\text { instanta- } \\
\text { neous } \\
\left(\mathrm{ft}^{3} / \mathbf{s}\right)\end{array}$ & $\begin{array}{c}\text { Sedi- } \\
\text { ment, } \\
\text { sus- } \\
\text { pended } \\
\text { (mg/L) }\end{array}$ & $\begin{array}{c}\text { Sedi- } \\
\text { ment, } \\
\text { sus- } \\
\text { pended, } \\
\text { diameter } \\
\text { (percent } \\
\text { finer } \\
\text { than } \\
0.062 \\
\text { mm) }\end{array}$ & $\begin{array}{l}\text { Spe- } \\
\text { cific } \\
\text { con- } \\
\text { duct- } \\
\text { ance, } \\
\text { field } \\
(\mu \mathrm{S} / \mathrm{cm})\end{array}$ & $\begin{array}{c}\text { pH, } \\
\text { field } \\
\text { (stan- } \\
\text { dard } \\
\text { units) }\end{array}$ & $\begin{array}{l}\text { Tem- } \\
\text { pera- } \\
\text { ture, } \\
\text { water } \\
\left({ }^{\circ} \mathrm{C}\right)\end{array}$ & $\begin{array}{c}\text { Hard- } \\
\text { ness } \\
\text { (mg/L } \\
\text { as } \\
\left.\mathrm{CaCO}_{3}\right)\end{array}$ \\
\hline
\end{tabular}

17 Bullion Mine tributary at mouth, near Basin

18 Jack Creek tributary near Basin

Jack Creek below Bullion Mine tributary, near Basin

Bullion Smelter tributary near Basin

21
Jack Creek above Bullion Mine tributary, near Basin

BASIN CREEK-Continued

$$
\text { 10-30-96 } 1200
$$

$05-16-97 \quad 1130$

09-25-97 1000

05-07-98 1715

09-24-98 0935

05-24-99 1100

08-02-99 1430

10-31-96 1600

05-16-97 1100

09-25-97 1110

02-11-98 1200

04-30-98 1400

05-07-98 1730

07-21-98 0900

07-22-98 1220

09-24-98 1005

05-24-99 1115

08-02-99 1310

08-03-99 1001

08-03-99 1301

10-30-96 1140

05-27-97 1230

07-22-98 1240

07-23-98 1115

07-24-98 1225

$10-31-96 \quad 1530$

05-16-97 1330

07-18-97 1500

09-25-97 1240

04-30-98 1415

05-07-98 1900

07-21-98 1024

07-25-98 1245

09-24-98 1130

05-24-99 1245

08-02-99 1145

08-03-99 1105

$10-30-96 \quad 1100$

05-27-97 1645

10-30-96 1435

05-13-97 1540

10-16-96 1240

05-21-97 1315
.75

8.3

.38

8.0

.42

6.6

.53

.40

5.6

.64

e. 19

1.0

3.9

1.0

--

.15

3.4

.24

$-$

$-$

1.2

16

16

$\cdots$

$$
-
$$$$
.87
$$$$
35
$$

35

$$
\begin{gathered}
4.4 \\
86
\end{gathered}
$$

5.0

20

7.0

$-$.

19

19

-.

.43

4.7

.10

2.4

3.0

233

-

--

-.

50

64

$-$

50

83

93

50

97
91

91
81

91

--

$-$

--

99
65

65
96

96

-.

$-$

$$
-
$$$$
\cdots
$$$$
-
$$$$
\cdots
$$$$
-
$$$$
-
$$$$
91
$$$$
\begin{aligned}
& 27 \\
& 79
\end{aligned}
$$$$
63
$$$$
71
$$$$
77
$$$$
55
$$$$
73
$$$$
\begin{array}{r}
73 \\
51
\end{array}
$$$$
\begin{aligned}
& 51 \\
& 86
\end{aligned}
$$

95

$\begin{array}{lll}-- & 0.0 & -\end{array}$

$\begin{array}{llll}95 & - & 0.0 & - \\ 64 & - & 1.5\end{array}$

$\begin{array}{llll}98 & 7.8 & 5.0 & 44\end{array}$

$\begin{array}{llll}60 & 7.7 & 2.0 & 25\end{array}$

$\begin{array}{llll}98 & 7.9 & 5.0 & 42\end{array}$

$\begin{array}{llll}65 & 8.0 & 2.5 & 27\end{array}$

$\begin{array}{rrrr}94 & 7.7 & 13.0 & 39\end{array}$

$\begin{array}{rrrr}238 & 5.9 & 0.0 & 86\end{array}$

$\begin{array}{llll}92 & 6.6 & 2.0 & 29\end{array}$

$\begin{array}{llll}228 & 5.4 & 6.0 & 77\end{array}$

$\begin{array}{llll}210 & 6.8 & 0.0 & 77\end{array}$

$\begin{array}{llll}110 & 7.1 & 0.0 & 39\end{array}$

$\begin{array}{llll}102 & 6.7 & 2.0 & 33\end{array}$

$\begin{array}{llll}120 & 7.4 & 8.5 & 39\end{array}$

$\begin{array}{llll}120 & 7.4 & 13.0\end{array}$

$\begin{array}{llll}266 & 5.6 & 4.0 & 87\end{array}$

$\begin{array}{llll}90 & 7.4 & 3.0 & 30\end{array}$

$\begin{array}{llll}203 & 5.5 & 13.5 & 66\end{array}$

$\begin{array}{llll}175 & 6.2 & 9.5 & -\end{array}$

$\begin{array}{llll}182 & 6.0 & 14.0\end{array}$

$84 \quad-.-0.0 \quad-$

$\begin{array}{llll}31 & -- & 2.0 & -\end{array}$

$\begin{array}{lll}49 & 7.7 & 12.0\end{array}$

$\begin{array}{lll}51 & 8.1 & 10.0\end{array}$

$\begin{array}{llll}51 & 8.0 & 11.0 & -\end{array}$

$\begin{array}{llll}132 & 7.7 & 0.0 & 49\end{array}$

$\begin{array}{llll}53 & 7.3 & 2.0 & 19\end{array}$

$\begin{array}{llll}83 & 7.5 & 11.5 & 30\end{array}$

$\begin{array}{llll}130 & 7.6 & 9.5 & 51\end{array}$

$\begin{array}{llll}94 & 7.5 & 0.0 & 38\end{array}$

$\begin{array}{llll}57 & 7.3 & 2.0 & 22\end{array}$

$\begin{array}{llll}76 & 7.7 & 11.0 & 29\end{array}$

$\begin{array}{llll}78 & 7.8 & 12.0 & --\end{array}$

$\begin{array}{llll}138 & 7.7 & 5.5 & 49\end{array}$

$\begin{array}{llll}60 & 7.5 & 4.5 & 21\end{array}$

$\begin{array}{llll}119 & 7.8 & 12.0 & 44\end{array}$

$\begin{array}{lllll}-. & - & - & -\end{array}$

$\begin{array}{llll}67 & - & 0.0 & -\end{array}$

$\begin{array}{llll}39 & - & 4.0 & -\end{array}$

$\begin{array}{llll}177 & -- & 0.0\end{array}$

$\begin{array}{llll}53 & - & 3.5 & -\end{array}$

$\begin{array}{llll}96 & 6.6 & 2.0 & 37\end{array}$

$\begin{array}{llll}39 & 7.2 & 6.5 & 12\end{array}$ 
Table 2. Water-quality data for streams in the Boulder River watershed, Montana, 1996-99 (Continued)

\begin{tabular}{|c|c|c|c|c|c|c|c|c|c|c|}
\hline $\begin{array}{c}\text { Site } \\
\text { num- } \\
\text { ber } \\
\text { (fig. 2) }\end{array}$ & Date & $\begin{array}{c}\text { Cal- } \\
\text { cium, } \\
\text { dissolved } \\
(\mathbf{m g} / \mathbf{L} \\
\text { as } \mathbf{C a})\end{array}$ & $\begin{array}{l}\text { Magne- } \\
\text { sium, } \\
\text { dissolved } \\
\text { (mg/L } \\
\text { as } \mathbf{M g} \text { ) }\end{array}$ & $\begin{array}{c}\text { Sodium, } \\
\text { dissolved } \\
(\mathbf{m g} / \mathbf{L} \\
\text { as } \mathbf{N a})\end{array}$ & $\begin{array}{l}\text { Potas- } \\
\text { sium, } \\
\text { dissolved } \\
\text { (mg/L } \\
\text { as K) }\end{array}$ & $\begin{array}{c}\mathrm{ANC}, \\
\text { lab } \\
(\mathrm{mg} / \mathrm{L} \\
\left.\text { as } \mathrm{CaCO}_{3}\right)\end{array}$ & $\begin{array}{c}\text { Sulfate, } \\
\text { dissolved } \\
(\mathrm{mg} / \mathrm{L} \\
\left.\text { as } \mathrm{SO}_{4}\right)\end{array}$ & $\begin{array}{c}\text { Chloride, } \\
\text { dissolved } \\
(\mathrm{mg} / \mathrm{L} \\
\text { as } \mathrm{Cl})\end{array}$ & $\begin{array}{c}\text { Fluoride, } \\
\text { dissolved } \\
\text { (mg/L } \\
\text { as F) }\end{array}$ & $\begin{array}{c}\text { Silica, } \\
\text { dissolved } \\
(\mathrm{mg} / \mathrm{L} \\
\left.\text { as } \mathrm{SiO}_{2}\right)\end{array}$ \\
\hline
\end{tabular}

\begin{tabular}{|c|c|c|c|c|c|c|c|c|c|c|c|}
\hline \multicolumn{12}{|c|}{ BASIN CREEK-Continued } \\
\hline \multirow[t]{7}{*}{16} & $10-30-96$ & - & -- & -- & -- & -- & -- & -- & -- & -- & -- \\
\hline & $05-16-97$ & -- & -- & -- & -- & -- & -- & -- & -- & -- & -- \\
\hline & $09-25-97$ & 13 & 2.8 & 2.6 & 1.1 & 39 & 10 & .36 & $<.10$ & 15 & 69 \\
\hline & $05-07-98$ & 7.6 & 1.5 & 1.7 & .86 & 23 & 5.6 & .29 & $<.10$ & 10 & 42 \\
\hline & $09-24-98$ & 12 & 2.6 & 2.7 & 1.2 & 39 & 10 & .23 & $<.10$ & 16 & 69 \\
\hline & $05-24-99$ & 7.9 & 1.7 & 1.8 & .90 & 24 & 6.5 & .24 & -- & - & - \\
\hline & 08-02-99 & 12 & 2.5 & 2.7 & 1.1 & 36 & 8.9 & $<.29$ & -- & -- & -- \\
\hline \multirow[t]{13}{*}{17} & $10-31-96$ & 24 & 6.4 & 3.2 & 1.1 & 1.3 & 100 & .40 & .10 & 21 & 166 \\
\hline & $05-16-97$ & 8.1 & 2.1 & 1.8 & .69 & 4.6 & 30 & .28 & $<.10$ & 13 & 61 \\
\hline & $09-25-97$ & 21 & 5.8 & 3.0 & 1.1 & $<1$ & 97 & .37 & .13 & 21 & 160 \\
\hline & $02-11-98$ & 21 & 5.8 & 3.1 & 1.0 & 6.2 & 84 & .29 & .12 & 20 & 146 \\
\hline & $04-30-98$ & 11 & 2.7 & 2.2 & .77 & 10 & 36 & .37 & $<.10$ & 14 & 76 \\
\hline & $05-07-98$ & 9.5 & 2.3 & 1.8 & .74 & 2.9 & 37 & .22 & $<.10$ & 12 & 68 \\
\hline & $07-21-98$ & 11 & 2.8 & 2.4 & .85 & 3.9 & 43 & .19 & $<.10$ & 16 & 82 \\
\hline & $07-22-98$ & -- & -- & -- & -- & -- & -- & -- & -- & -- & -- \\
\hline & $09-24-98$ & 24 & 6.7 & 3.3 & 1.2 & - & 120 & .45 & .13 & 23 & - \\
\hline & $05-24-99$ & 8.3 & 2.2 & 1.9 & .70 & 6.4 & 29 & .28 & -- & -- & -- \\
\hline & 08-02-99 & 18 & 5.0 & 3.0 & 1.2 & 1.3 & 84 & .29 & -- & - & -- \\
\hline & 08-03-99 & - & -- & -- & -. & -- & -- & -- & -- & -- & -- \\
\hline & 08-03-99 & -- & -- & -- & -- & -- & -- & -- & -- & -- & -- \\
\hline \multirow[t]{5}{*}{18} & $10-30-96$ & - & -- & -- & -- & -- & -- & -- & -- & -- & -- \\
\hline & $05-27-97$ & - & -- & -- & -- & -- & -- & -- & -- & - & -- \\
\hline & $07-22-98$ & -- & -- & -- & -- & -- & -- & -- & -- & -- & -- \\
\hline & $07-23-98$ & -- & -- & -- & -- & -- & -- & -- & - & -- & -- \\
\hline & $07-24-98$ & -- & -- & -- & -- & -- & -- & -- & -- & -- & -- \\
\hline \multirow[t]{12}{*}{19} & $10-31-96$ & 14 & 3.5 & 3.0 & 1.1 & 24 & 36 & .40 & $<.10$ & 18 & 92 \\
\hline & $05-16-97$ & 5.3 & 1.3 & 1.6 & .78 & 12 & 9.8 & .27 & $<.10$ & 12 & 39 \\
\hline & $07-18-97$ & 8.7 & 2.0 & 2.2 & 1.0 & 21 & 17 & .19 & $<.10$ & 15 & 60 \\
\hline & $09-25-97$ & 14 & 3.6 & 2.9 & 1.3 & 23 & 34 & .34 & .10 & 18 & 90 \\
\hline & $04-30-98$ & 11 & 2.4 & 2.2 & .98 & 26 & 17 & .33 & $<.10$ & 12 & 63 \\
\hline & $05-07-98$ & 6.4 & 1.5 & 1.6 & .84 & 14 & 11 & .22 & $<.10$ & 11 & 41 \\
\hline & $07-21-98$ & 8.4 & 1.9 & 2.2 & .93 & 22 & 11 & .21 & $<.10$ & 15 & 54 \\
\hline & $07-25-98$ & -- & -- & -- & -- & -- & -- & - & -- & -- & -- \\
\hline & $09-24-98$ & 14 & 3.5 & 3.1 & 1.3 & 22 & 36 & .31 & .10 & 19 & 92 \\
\hline & $05-24-99$ & 6.1 & 1.5 & 1.7 & .86 & 15 & 11 & .25 & -- & -- & -- \\
\hline & 08-02-99 & 12 & 3.1 & 2.9 & .12 & 22 & 30 & $<.29$ & -- & - & -- \\
\hline & $08-03-99$ & -- & -- & -- & - & -- & -- & -- & -- & -- & -- \\
\hline \multirow[t]{2}{*}{20} & $10-30-96$ & -- & -- & -- & -- & -- & -- & -- & - & -- & - \\
\hline & $05-27-97$ & -- & -- & -- & -- & -- & -- & -- & -- & -- & -- \\
\hline \multirow[t]{2}{*}{21} & $10-30-96$ & -- & -- & -- & -- & -- & -- & -- & - & -- & - \\
\hline & $05-13-97$ & -- & -- & -- & -- & -- & -- & -- & -- & -- & - \\
\hline \multirow[t]{2}{*}{22} & $10-16-96$ & 11 & 2.4 & 3.4 & 1.2 & 27 & 16 & 1.1 & $<.10$ & 17 & 69 \\
\hline & $05-21-97$ & 3.3 & .76 & 1.8 & .83 & 6.4 & 5.4 & 2.7 & $<.10$ & 13 & 32 \\
\hline
\end{tabular}


Table 2. Water-quality data for streams in the Boulder River watershed, Montana, 1996-99 (Continued)

\begin{tabular}{|c|c|c|c|c|c|c|c|}
\hline $\begin{array}{c}\text { Site } \\
\text { num- } \\
\text { ber } \\
\text { (fig. 2) }\end{array}$ & Date & $\begin{array}{c}\text { Nitrite, } \\
\text { dissolved } \\
\text { (mg/L } \\
\text { as } \mathbf{N})\end{array}$ & $\begin{array}{l}\text { Nitrite } \\
\text { plus } \\
\text { nitrate, } \\
\text { dissolved } \\
\text { (mg/L } \\
\text { as } N \text { ) }\end{array}$ & $\begin{array}{c}\text { Ammonia, } \\
\text { dissolved } \\
\text { (mg/L } \\
\text { as } N)\end{array}$ & $\begin{array}{c}\text { Ammonia plus } \\
\text { organic } \\
\text { nitrogen, } \\
\text { total } \\
(\mathrm{mg} / \mathrm{L} \\
\text { as } \mathrm{N})\end{array}$ & $\begin{array}{l}\text { Phos- } \\
\text { phorus, } \\
\text { total } \\
\text { (mg/L } \\
\text { as P) }\end{array}$ & $\begin{array}{l}\text { Phos- } \\
\text { phorus, } \\
\text { ortho- } \\
\text { phosphate, } \\
\text { dissolved } \\
\text { (mg/L } \\
\text { as P) }\end{array}$ \\
\hline \multicolumn{8}{|c|}{ BASIN CREEK-Continued } \\
\hline \multirow[t]{7}{*}{16} & $10-30-96$ & - & -- & -- & -- & -- & -- \\
\hline & $05-16-97$ & -- & -- & -- & -- & -- & -- \\
\hline & $09-25-97$ & $<.01$ & $<.05$ & $<.015$ & $<.2$ & $<.010$ & $<.010$ \\
\hline & $05-07-98$ & - & - & -- & -- & - & - \\
\hline & $09-24-98$ & - & - & -- & -- & -- & -- \\
\hline & $05-24-99$ & $\sim$ & - & -- & -- & -. & - \\
\hline & $08-02-99$ & -- & -- & -- & -- & -- & -- \\
\hline \multirow[t]{13}{*}{17} & $10-31-96$ & - & -- & -- & $\ldots$ & -- & -- \\
\hline & $05-16-97$ & - & -- & -- & -- & - & - \\
\hline & $09-25-97$ & $<.01$ & $<.05$ & $<.015$ & $<.2$ & $<.010$ & $<.010$ \\
\hline & $02-11-98$ & $<.01$ & .11 & .039 & -- & -- & .012 \\
\hline & $04-30-98$ & -- & - & -- & -- & -- & -- \\
\hline & $05-07-98$ & - & -- & -- & -- & -. & - \\
\hline & $07-21-98$ & $<.01$ & .05 & .038 & -- & -- & $<.010$ \\
\hline & $07-22-98$ & -- &.- & -- & -- & -- & -- \\
\hline & $09-24-98$ & -- & -- & -- & - & -- & -- \\
\hline & $05-24-99$ & -- & -- & -- & -- & -- & -- \\
\hline & $08-02-99$ & - & - & -. & -- & -- & -- \\
\hline & $08-03-99$ & -- & -- & -- & -- & -- & -. \\
\hline & $08-03-99$ & - & -- & -- & -- & -- & -- \\
\hline \multirow[t]{5}{*}{18} & $10-30-96$ & -- & -- & - & -- & -- & - \\
\hline & $05-27-97$ & -- & -- & -- & - & -- & -- \\
\hline & $07-22-98$ & -- & -- & -- & -- & -- & - \\
\hline & $07-23-98$ & -- & -- & -- & -- & -- & -. \\
\hline & $07-24-98$ & -. & -- & -- & -- & -- & -- \\
\hline \multirow[t]{12}{*}{19} & $10-31-96$ & - & -- & -- & -- & -- & -- \\
\hline & $05-16-97$ &.- & -- & -- & -- & -- & -- \\
\hline & $07-18-97$ & $<.01$ & $<.05$ & $<.015$ & -- & -- & $<.010$ \\
\hline & $09-25-97$ & $<.01$ & $<.05$ & $<.015$ & $<.2$ & $<.010$ & $<.010$ \\
\hline & $04-30-98$ & $\ldots$ & -- & -- & -- & -- & -- \\
\hline & $05-07-98$ & -. & -- & -- & -- & -- & -- \\
\hline & $07-21-98$ & $<.01$ & $<.05$ & .036 & - & -- & $<.010$ \\
\hline & $07-25-98$ & -- & -- & -- & -- & -- & -- \\
\hline & $09-22-98$ & -- & -- & -- & -. & -. & -- \\
\hline & $05-24-99$ & -. & -- & -. & -- & -. & .. \\
\hline & 08-02-99 & - & -- & -- & -- & - & -- \\
\hline & 08-03-99 & -- & -- & -- & -- & -. & -- \\
\hline \multirow[t]{2}{*}{20} & $10-30-96$ & -- & -- & -- & -- & -- & -- \\
\hline & $05-27-97$ & - & -- & -- & -- & -- & -- \\
\hline \multirow[t]{2}{*}{21} & $10-30-96$ & -- & -- & -- & -- & -- & -- \\
\hline & $05-13-97$ & - & -- & -- & -- & -- & -- \\
\hline \multirow[t]{2}{*}{22} & $10-16-96$ & -- & -- & -- & -- & -- & -- \\
\hline & $05-21-97$ & - & - & - & -- & -- & -- \\
\hline
\end{tabular}


Table 2. Water-quality data for streams in the Boulder River watershed, Montana, 1996-99 (Continued)

\begin{tabular}{|c|c|c|c|c|c|c|c|c|c|c|c|c|}
\hline $\begin{array}{c}\text { Site } \\
\text { num- } \\
\text { ber } \\
\text { (fig. 2) }\end{array}$ & Date & $\begin{array}{l}\text { Alumi- } \\
\text { num, } \\
\text { total } \\
\text { recov- } \\
\text { erable } \\
(\mu \mathrm{g} / \mathrm{L} \text { as } \\
\mathrm{Al})\end{array}$ & $\begin{array}{l}\text { Alumi- } \\
\text { num, } \\
\text { dis- } \\
\text { solved } \\
(\mu \mathrm{g} / \mathrm{L} \text { as } \\
\text { Al) }\end{array}$ & $\begin{array}{l}\text { Anti- } \\
\text { mony, } \\
\text { dis- } \\
\text { solved } \\
(\mu \mathrm{g} / \mathrm{L} \text { as } \\
\text { Sb) }\end{array}$ & $\begin{array}{c}\text { Arsenic, } \\
\text { total } \\
\text { recov- } \\
\text { erable } \\
(\mu \mathrm{g} / \mathrm{L} \text { as } \\
\text { As) }\end{array}$ & $\begin{array}{l}\text { Arsenic, } \\
\text { dis- } \\
\text { solved } \\
(\mu \mathrm{g} / \mathrm{L} \text { as } \\
\text { As) }\end{array}$ & $\begin{array}{l}\text { Barium, } \\
\text { dis- } \\
\text { solved } \\
(\mu \mathrm{g} / \mathrm{L} \text { as } \\
\text { Ba) }\end{array}$ & $\begin{array}{c}\text { Beryl- } \\
\text { lium, } \\
\text { dis- } \\
\text { solved } \\
(\mu \mathrm{g} / \mathrm{L} \text { as } \\
\text { Be) }\end{array}$ & $\begin{array}{c}\text { Cad- } \\
\text { mium, } \\
\text { total } \\
\text { recov- } \\
\text { erable } \\
(\mu \mathrm{g} / \mathrm{L} \text { as } \\
\text { Cd) }\end{array}$ & $\begin{array}{c}\text { Cad- } \\
\text { mium, } \\
\text { dis- } \\
\text { solved } \\
(\mu \mathrm{g} / \mathrm{L} \text { as } \\
\text { Cd) }\end{array}$ & $\begin{array}{c}\text { Chro- } \\
\text { mium, } \\
\text { total } \\
\text { recov- } \\
\text { erable } \\
(\mu \mathrm{g} / \mathrm{L} \text { as } \\
\mathrm{Cr})\end{array}$ & $\begin{array}{c}\text { Chro- } \\
\text { mium, } \\
\text { dis- } \\
\text { solved } \\
(\mu \mathrm{g} / \mathrm{L} \text { as } \\
\text { Cr) }\end{array}$ \\
\hline
\end{tabular}

\begin{tabular}{|c|c|c|c|c|c|c|c|c|c|c|c|c|}
\hline \multirow[b]{2}{*}{16} & \multicolumn{12}{|c|}{ BASIN CREEK--Continued } \\
\hline & $10-30-96$ & - & - & - & -- & -- & -- & -- & - & - & -- & -- \\
\hline & $05-16-97$ & -- & -- & -- & -- & -- & -- & -- & - & - & - & -- \\
\hline & $09-25-97$ & 40 & 3.2 & $<1$ & 6 & 5 & 9.8 & $<1$ & $<1$ & .1 & -- & $<1$ \\
\hline & $05-07-98$ & 390 & 29 & $<1$ & 7 & 3 & 5.9 & $<1$ & $<1$ & $<.3$ & -- & $<1$ \\
\hline & $09-24-98$ & 20 & 3.2 & $<1$ & 6 & 6 & 10 & $<1$ & $<1$ & $<.3$ & - & $<1$ \\
\hline & $05-24-99$ & 450 & 31 & -- & 12 & 5 & -- & - & $<1$ & $<.3$ & -- & - \\
\hline \multirow{8}{*}{17} & $05-16-97$ & 1,300 & 59 & $<1$ & 100 & 2 & 11 & $<1$ & 16 & 15 & $<1$ & $<1$ \\
\hline & $09-25-97$ & 1,500 & 284 & $<1$ & 62 & $<1$ & 23 & $<1$ & 49 & 48 & -- & $<1$ \\
\hline & $02-11-98$ & 860 & 21 & $<1$ & 5 & $<1$ & 18 & $<1$ & 38 & 39 & -- & $<1$ \\
\hline & $04-30-98$ & 870 & 127 & $<1$ & 28 & 3 & 11 & $<1$ & 16 & 16 & -- & $<1$ \\
\hline & $05-07-98$ & 3,300 & 47 & 1 & 440 & 3 & 13 & $<1$ & 17 & 17 & -- & $<1$ \\
\hline & $08-02-99$ & 1,300 & 306 & -- & 97 & 1 & -- & -- & 39 & 38 & -. & -- \\
\hline & 08-03-99 & -- & -- & -- & -- & -- & -- & -- & -- & 33 & - & -- \\
\hline & 08-03-99 & - & - & $\cdots$ & $\sim$ & -- & -- & - & -- & 35 & - & -- \\
\hline \multirow[t]{5}{*}{18} & $10-30-96$ & -- & -- & -- & -- & -- & -- & -- & -- & -- & - & -. \\
\hline & $05-27-97$ & - & -- & -- & - & -- & -- & -- & -- & -- & - & -- \\
\hline & $07-22-98$ & -- & -- & -- & - & -- & - & -- & -- & $<.1$ & - & -- \\
\hline & $07-23-98$ & -- & -- & -- & -- & -- & -- & -- & - & $<.1$ & - & - \\
\hline & $07-24-98$ & -- & -- & -- & -- & -- & -- & -- & -- & $<.1$ & - & -- \\
\hline \multirow[t]{5}{*}{19} & $10-31-96$ & -- & 37 & $<1$ & 6 & 2 & 18 & $<1$ & 11 & 11 & $<1$ & $<1$ \\
\hline & $05-16-97$ & 1,000 & 63 & $<1$ & 32 & 2 & 13 & $<1$ & 3 & 2.5 & $<1$ & $<1$ \\
\hline & $07-18-97$ & -- & 21 & $<1$ & 32 & 3 & 13 & $<1$ & 5 & 4.1 & - & $<1$ \\
\hline & $08-02-99$ & 280 & 63 & -- & 23 & 2 & -- & -- & 10 & 8.6 & -- & - \\
\hline & 08-03-99 & -- & -- & - & -- & -- & -- & -- & -- & 7.3 & -- & -- \\
\hline \multirow[t]{2}{*}{20} & $10-30-96$ & - & - & -- & -- & -- & -- & -- & -- & - & - & -- \\
\hline & $05-27-97$ & - & -- & -- & -- & -- & -- & -- & -- & -- & -- & - \\
\hline \multirow[t]{2}{*}{21} & $10-30-96$ & -- & -- & -- & - & -- & -- & - & - & -- & -- & - \\
\hline & $05-13-97$ & - & -- & -- & -- & - & -- & -- & - & -- & -. & -- \\
\hline \multirow[t]{2}{*}{22} & $10-16-96$ & -- & 7.5 & $<1$ & 9 & 7 & 24 & $<1$ & $<1$ & .6 & $<1$ & $<1$ \\
\hline & $05-21-97$ & 570 & 98 & $<1$ & 12 & 4 & 13 & $<1$ & $<1$ & .5 & $<1$ & $<1$ \\
\hline
\end{tabular}


Table 2. Water-quality data for streams in the Boulder River watershed, Montana, 1996-99 (Continued)

\begin{tabular}{|c|c|c|c|c|c|c|c|c|c|c|c|}
\hline $\begin{array}{c}\text { Site } \\
\text { num- } \\
\text { ber } \\
\text { (fig. 2) }\end{array}$ & Date & $\begin{array}{c}\text { Cobalt, } \\
\text { dis- } \\
\text { solved } \\
(\mu \mathrm{g} / \mathrm{L} \mathrm{as} \\
\text { Co })\end{array}$ & $\begin{array}{l}\text { Copper, } \\
\text { total recov- } \\
\text { erable } \\
(\mu \mathrm{g} / \mathrm{L} \text { as } \\
\mathrm{Cu})\end{array}$ & $\begin{array}{c}\text { Copper, } \\
\text { dis- } \\
\text { solved } \\
(\mu \mathrm{g} / \mathrm{L} \text { as } \\
\text { Cu })\end{array}$ & $\begin{array}{c}\text { Iron, } \\
\text { total } \\
\text { recov- } \\
\text { erable } \\
(\mu \mathrm{g} / \mathrm{L} \text { as } \\
\mathrm{Fe})\end{array}$ & $\begin{array}{c}\text { Iron, } \\
\text { dis- } \\
\text { solved } \\
(\mu \mathrm{g} / \mathrm{L} \text { as } \\
\text { Fe) }\end{array}$ & $\begin{array}{l}\text { Lead, } \\
\text { total } \\
\text { recov- } \\
\text { erable } \\
(\mu \mathrm{g} / \mathrm{L} \text { as } \\
\mathrm{Pb})\end{array}$ & $\begin{array}{c}\text { Lead, } \\
\text { dis- } \\
\text { solved } \\
(\mu \mathrm{g} / \mathrm{L} \text { as } \\
\text { Pb) }\end{array}$ & $\begin{array}{c}\text { Lithium, } \\
\text { dis- } \\
\text { solved } \\
(\mu \mathrm{g} / \mathrm{L} \mathrm{as} \\
\mathrm{Li})\end{array}$ & $\begin{array}{c}\text { Manga- } \\
\text { nese, } \\
\text { total } \\
\text { recov- } \\
\text { erable } \\
(\mu \mathrm{g} / \mathrm{L} \text { as } \\
\mathrm{Mn})\end{array}$ & $\begin{array}{c}\text { Manga- } \\
\text { nese, } \\
\text { dis- } \\
\text { solved } \\
(\mu \mathrm{g} / \mathrm{L} \text { as } \\
\mathrm{Mn})\end{array}$ \\
\hline
\end{tabular}

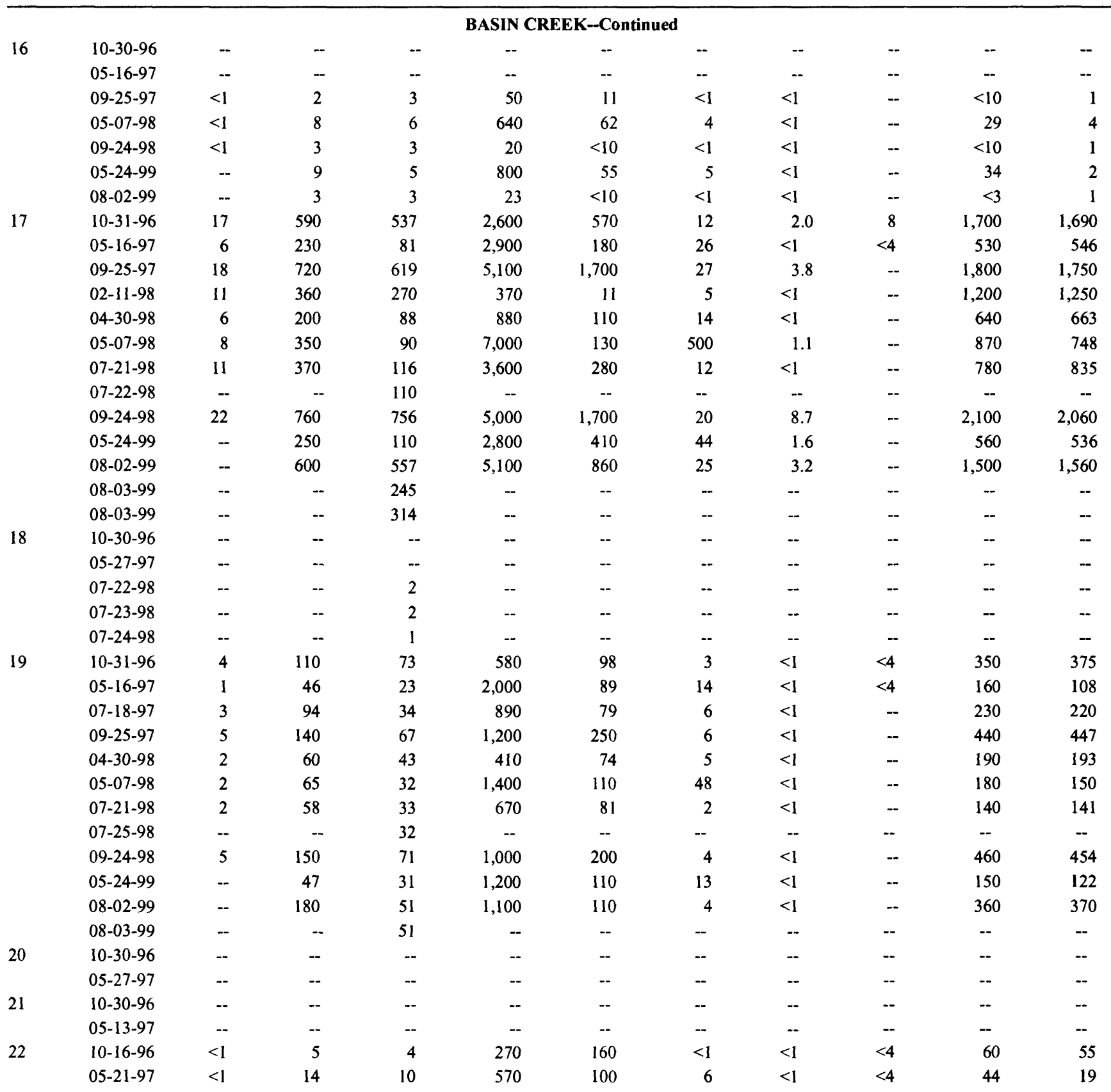


Table 2. Water-quality data for streams in the Boulder River watershed, Montana, 1996-99 (Continued)

\begin{tabular}{|c|c|c|c|c|c|c|c|c|c|c|c|}
\hline $\begin{array}{c}\text { Site } \\
\text { num- } \\
\text { ber } \\
\text { (fig. 2) }\end{array}$ & Date & $\begin{array}{c}\text { Mercury, } \\
\text { total } \\
\text { recov- } \\
\text { erable } \\
(\mu \mathrm{g} / \mathrm{L} \text { as } \\
\mathrm{Hg})\end{array}$ & $\begin{array}{c}\text { Mercury, } \\
\text { dis- } \\
\text { solved } \\
(\mu \mathrm{g} / \mathrm{L} \text { as } \\
\mathrm{Hg})\end{array}$ & $\begin{array}{c}\text { Molyb- } \\
\text { denum, } \\
\text { dis- } \\
\text { solved } \\
(\mu \mathrm{g} / \mathrm{L} \text { as } \\
\text { Mo) }\end{array}$ & $\begin{array}{l}\text { Nickel, } \\
\text { total } \\
\text { recov- } \\
\text { erable } \\
(\mu \mathrm{g} / \mathrm{L} \text { as } \\
\mathrm{Ni})\end{array}$ & $\begin{array}{c}\text { Nickel, } \\
\text { dis- } \\
\text { solved } \\
(\mu \mathrm{g} / \mathrm{L} \text { as } \\
\mathrm{Ni})\end{array}$ & $\begin{array}{l}\text { Silver, } \\
\text { total } \\
\text { recov- } \\
\text { erable } \\
(\mu \mathrm{g} / \mathrm{L} \text { as } \\
\mathrm{Ag})\end{array}$ & $\begin{array}{l}\text { Silver, } \\
\text { dis- } \\
\text { solved } \\
(\mu \mathrm{g} / \mathrm{L} \text { as } \\
\mathrm{Ag})\end{array}$ & $\begin{array}{c}\text { Uranium, } \\
\text { dis- } \\
\text { solved } \\
(\mu \mathrm{g} / \mathrm{L} \text { as } \\
\mathrm{U})\end{array}$ & $\begin{array}{c}\text { Zinc, } \\
\text { total } \\
\text { recov- } \\
\text { erable } \\
(\mu \mathrm{g} / \mathrm{L} \text { as } \\
\mathrm{Zn})\end{array}$ & $\begin{array}{c}\text { Zinc, } \\
\text { dis- } \\
\text { solved } \\
(\mu \mathrm{g} / \mathrm{L} \text { as } \\
\text { Zn) }\end{array}$ \\
\hline
\end{tabular}

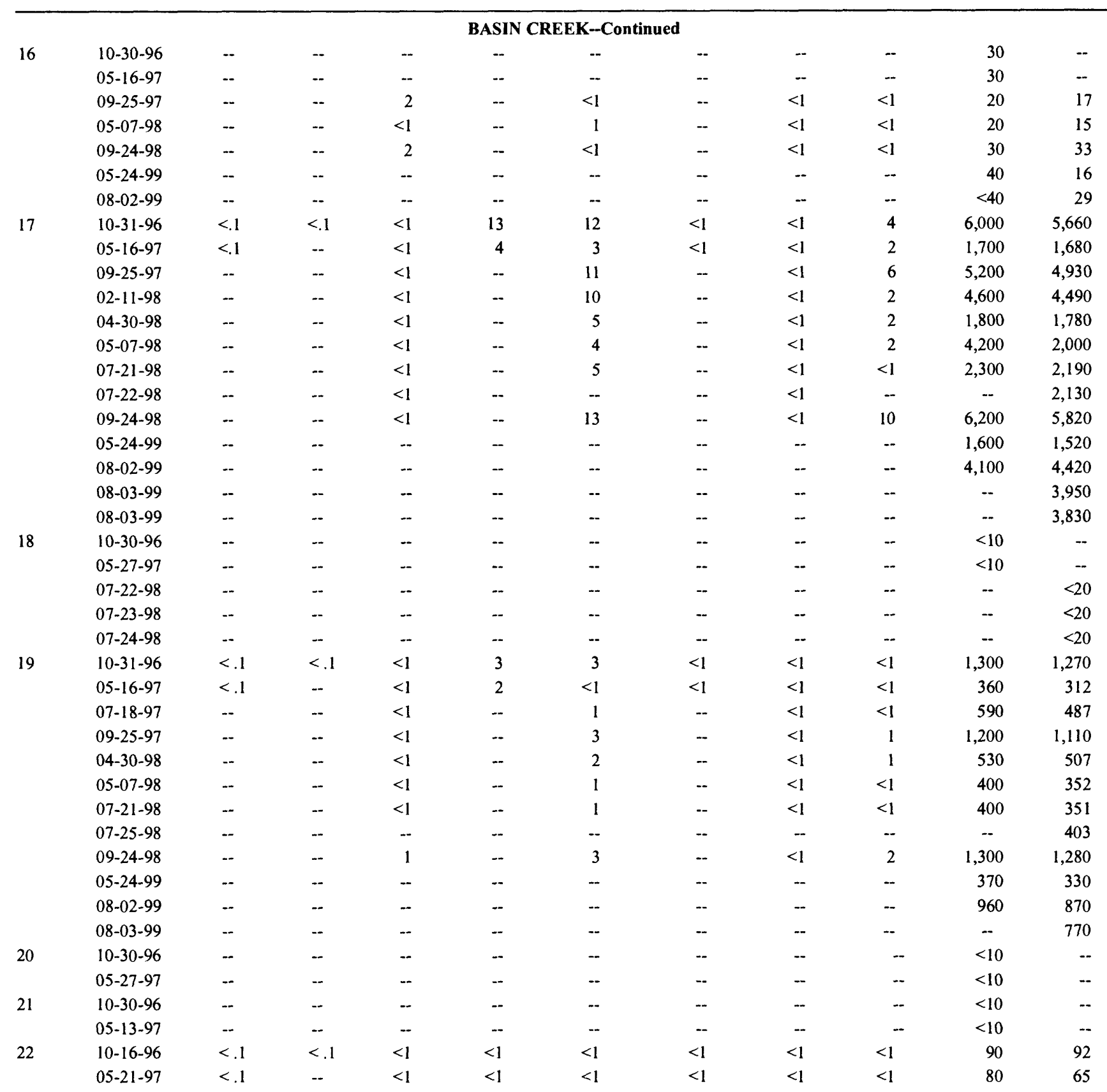


Table 2. Water-quality data for streams in the Boulder River watershed, Montana, 1996-99 (Continued)

\begin{tabular}{|c|c|c|c|c|c|c|c|c|c|c|}
\hline $\begin{array}{c}\text { Site } \\
\text { num- } \\
\text { ber } \\
\text { (fig. 2) } \\
.\end{array}$ & Station name & Date & Time & $\begin{array}{c}\text { Dis- } \\
\text { charge, } \\
\text { instanta- } \\
\text { neous } \\
\left(\mathrm{ft}^{3} / \mathbf{s}\right)\end{array}$ & $\begin{array}{c}\text { Sedi- } \\
\text { ment, } \\
\text { sus- } \\
\text { pended } \\
\text { (mg/L) }\end{array}$ & $\begin{array}{c}\text { Sedi- } \\
\text { ment, } \\
\text { sus- } \\
\text { pended, } \\
\text { diameter } \\
\text { (percent } \\
\text { finer } \\
\text { than } \\
\mathbf{0 . 0 6 2} \\
\text { mm) }\end{array}$ & $\begin{array}{c}\text { Spe- } \\
\text { cific } \\
\text { con- } \\
\text { duct- } \\
\text { ance, } \\
\text { field } \\
(\mu S / \mathrm{cm})\end{array}$ & $\begin{array}{c}\text { pH, } \\
\text { field } \\
\text { (stan- } \\
\text { dard } \\
\text { units) }\end{array}$ & $\begin{array}{l}\text { Tem- } \\
\text { pera- } \\
\text { ture, } \\
\text { water } \\
\left({ }^{\circ} \mathrm{C}\right)\end{array}$ & $\begin{array}{c}\text { Hard- } \\
\text { ness } \\
(\mathrm{mg} / \mathrm{L} \\
\text { as } \\
\left.\mathrm{CaCO}_{3}\right)\end{array}$ \\
\hline
\end{tabular}

$31 \quad$ Overland Creek near Basin

32 Cataract Creek above Rocker Creek, near Basin

Cataract Creek above Nellie Grant Creek, near Basin

Cataract Creek tributary above Nellie Grant Creek, near Basin

Cataract Creek 100 feet above Nellie Grant Creek, near Basin

Nellie Grant Creek near Basin

Cataract Creek below Nellie Grant Creek, near Basin

Creek, near Basin

\section{BASIN CREEK-Continued}

Saul Haggarty Gulch at mouth, near Basin

Basin Creek at Basin

\begin{tabular}{ccc}
\multicolumn{3}{c}{ BASIN CREEK-Continued } \\
$10-30-96$ & 1450 & .20 \\
$05-13-97$ & 1445 & 8.8 \\
$10-15-96$ & 1545 & 3.9 \\
$12-03-96$ & 1245 & 3.9 \\
$02-11-97$ & 1135 & 4.0 \\
$04-18-97$ & 1140 & 15 \\
$05-08-97$ & 0945 & 45 \\
$05-21-97$ & 1630 & 251 \\
$06-03-97$ & 1015 & 173 \\
$06-11-97$ & 1030 & 211 \\
$06-17-97$ & 1030 & 106 \\
$07-18-97$ & 1300 & 26 \\
$09-23-97$ & 1050 & 5.8 \\
$02-09-98$ & 1400 & 4.2 \\
$04-29-98$ & 1045 & 48 \\
$05-05-98$ & 1015 & 136 \\
$09-23-98$ & 1545 & 4.4 \\
$05-25-99$ & 1120 & 188 \\
$09-14-99$ & 1030 & 3.1
\end{tabular}

$\begin{array}{rrrrr}-- & 122 & -- & 0.0 & - \\ -- & 56 & -- & 7.0 & - \\ 61 & 104 & 7.6 & 5.5 & 42 \\ 56 & -- & 7.5 & 0.0 & 39 \\ 72 & 108 & 7.7 & 0.0 & 42 \\ 67 & 100 & 7.9 & 4.5 & 35 \\ 75 & 67 & 7.6 & 2.5 & 25 \\ 42 & 38 & 7.6 & 10.0 & 13 \\ 36 & 38 & 7.0 & 7.5 & 13 \\ 38 & 40 & 7.4 & 9.5 & 14 \\ 42 & 50 & 7.5 & 11.0 & 18 \\ 83 & 73 & 7.7 & 13.5 & 25 \\ 42 & 107 & 7.8 & 8.0 & 39 \\ 71 & 105 & 7.8 & 0.0 & 40 \\ 85 & 62 & 7.9 & 4.5 & 23 \\ 62 & 41 & 7.7 & 3.5 & 14 \\ 52 & 100 & 8.2 & 11.5 & 40 \\ 47 & 36 & 7.4 & 5.5 & 12 \\ 56 & 106 & 7.8 & 8.5 & 40\end{array}$

\section{CATARACT CREEK}

$\begin{array}{cccc}10-18-96 & 1143 & .30 \\ 09-26-97 & 1320 & .22 & .10 \\ 10-18-96 & 1142 & .03 & - \\ 09-26-97 & 1340 & .22 & - \\ 05-28-97 & 1320 & 20 & .35 \\ 09-26-97 & 1325 & .1 & - \\ 09-22-98 & 0955 & 7.4 & - \\ 10-18-96 & 1145 & .01 & \\ 05-28-97 & 1400 & 2.0 & - \\ 09-22-98 & 1045 & .37 & - \\ 10-18-96 & 1135 & .3 & - \\ 05-28-97 & 1430 & 28 & - \\ 09-26-97 & 1415 & .08 & - \\ 05-28-97 & 1115 & 7.3 & - \\ 09-26-97 & 1240 & & - \\ 10-18-96 & 0945 & .68 & \end{array}$

$\begin{array}{rr}-- & - \\ -- & - \\ 1 & 61 \\ 1 & 56 \\ 2 & 72 \\ 13 & 67 \\ 10 & 75 \\ 35 & 42 \\ 26 & 36 \\ 74 & 38 \\ 17 & 42 \\ 2 & 83 \\ 1 & 42 \\ 1 & 71 \\ 12 & 85 \\ 29 & 62 \\ 3 & 52 \\ 45 & 47 \\ 2 & 56\end{array}$

$\begin{array}{rrrrrr}-- & -- & 83 & -- & 0.0 & -- \\ -- & -- & 79 & -- & 8.5 & -- \\ -- & -- & 126 & - & .5 & - \\ -- & -- & 103 & -- & 10.0 & - \\ -- & -- & 25 & -- & 5.5 & - \\ -- & -- & 82 & -- & 9.5 & -- \\ 3 & 64 & 74 & 7.7 & 2.5 & 36 \\ -- & -- & 133 & -- & 1.0 & -- \\ -- & -- & 23 & -- & 13.5 & - \\ 1 & 90 & 102 & 7.0 & 4.5 & 49 \\ -- & -- & 91 & -- & .5 & - \\ -- & -- & 25 & -- & 8.5 & -- \\ -- & -- & 85 & -- & 10.0 & - \\ -- & -- & 27 & -- & 3.5 & - \\ -- & -- & 76 & -- & 9.0 & - \\ -- & -- & 89 & -- & 1.0 & - \\ -- & -- & 33 & -- & 4.0 & -\end{array}$


Table 2. Water-quality data for streams in the Boulder River watershed, Montana, 1996-99 (Continued)

\begin{tabular}{|c|c|c|c|c|c|c|c|c|c|c|}
\hline $\begin{array}{c}\text { Site } \\
\text { num- } \\
\text { ber } \\
\text { (fig. 2) }\end{array}$ & Date & $\begin{array}{c}\text { Cal- } \\
\text { cium, } \\
\text { dissolved } \\
(\mathrm{mg} / \mathrm{L} \\
\text { as Ca) }\end{array}$ & $\begin{array}{l}\text { Magne- } \\
\text { sium, } \\
\text { dissolved } \\
\text { (mg/L } \\
\text { as } \mathbf{M g})\end{array}$ & $\begin{array}{c}\text { Sodium, } \\
\text { dissolved } \\
(\mathrm{mg} / \mathrm{L} \\
\text { as } \mathrm{Na})\end{array}$ & $\begin{array}{l}\text { Potas- } \\
\text { sium, } \\
\text { dissolved } \\
\text { (mg/L } \\
\text { as K) }\end{array}$ & $\begin{array}{c}\mathrm{ANC}, \\
\text { lab } \\
(\mathrm{mg} / \mathrm{L} \\
\left.\text { as } \mathrm{CaCO}_{3}\right)\end{array}$ & $\begin{array}{c}\text { Sulfate, } \\
\text { dissolved } \\
(\mathrm{mg} / \mathrm{L} \\
\left.\text { as } \mathrm{SO}_{4}\right)\end{array}$ & $\begin{array}{c}\text { Chloride, } \\
\text { dissolved } \\
(\mathrm{mg} / \mathrm{L} \\
\text { as } \mathrm{Cl})\end{array}$ & $\begin{array}{c}\text { Fluoride, } \\
\text { dissolved } \\
\text { (mg/L } \\
\text { as F) }\end{array}$ & $\begin{array}{c}\text { Silica, } \\
\text { dissolved } \\
(\mathrm{mg} / \mathrm{L} \\
\left.\text { as } \mathrm{SiO}_{2}\right)\end{array}$ \\
\hline
\end{tabular}

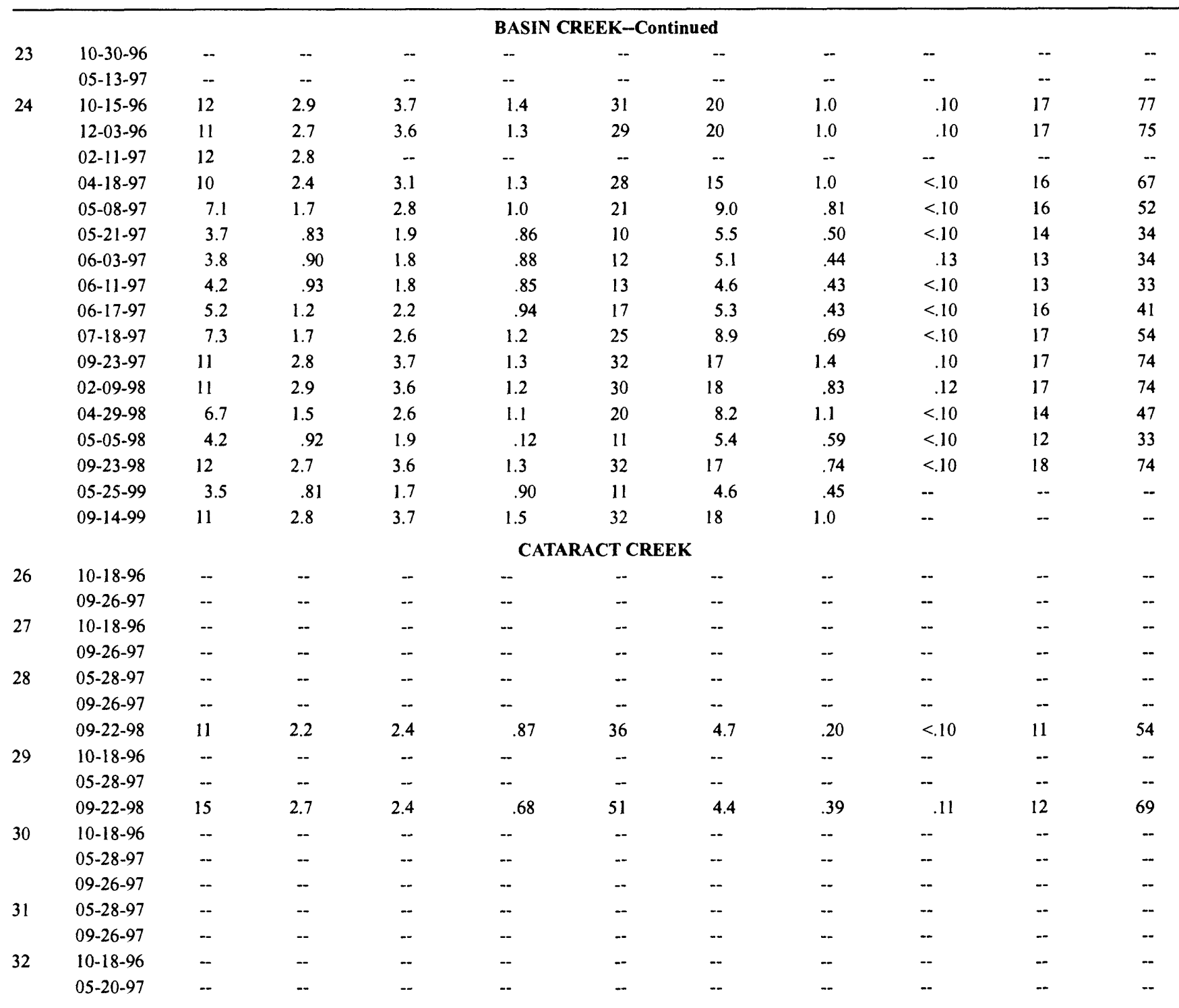


Table 2. Water-quality data for streams in the Boulder River watershed, Montana, 1996-99 (Continued)

\begin{tabular}{|c|c|c|c|c|c|c|c|}
\hline $\begin{array}{c}\text { Site } \\
\text { num- } \\
\text { ber } \\
\text { (fig. 2) }\end{array}$ & Date & $\begin{array}{c}\text { Nitrite, } \\
\text { dissolved } \\
\text { (mg/L } \\
\text { as } \mathbf{N})\end{array}$ & $\begin{array}{l}\text { Nitrite } \\
\text { plus } \\
\text { nitrate, } \\
\text { dissolved } \\
(\mathrm{mg} / \mathrm{L} \\
\text { as } \mathrm{N})\end{array}$ & $\begin{array}{c}\text { Ammonia, } \\
\text { dissolved } \\
\text { (mg/L } \\
\text { as } N \text { ) }\end{array}$ & $\begin{array}{c}\text { Ammonia plus } \\
\text { organic } \\
\text { nitrogen, } \\
\text { total } \\
(\mathrm{mg} / \mathrm{L} \\
\text { as } \mathrm{N})\end{array}$ & $\begin{array}{c}\text { Phos- } \\
\text { phorus, } \\
\text { total } \\
\text { (mg/L } \\
\text { as P) }\end{array}$ & $\begin{array}{l}\text { Phos- } \\
\text { phorus, } \\
\text { ortho- } \\
\text { phosphate, } \\
\text { dissolved } \\
\text { (mg/L } \\
\text { as P) }\end{array}$ \\
\hline
\end{tabular}

\begin{tabular}{|c|c|c|c|c|c|c|c|}
\hline \multicolumn{8}{|c|}{ BASIN CREEK-Continued } \\
\hline \multirow[t]{2}{*}{23} & $10-30-96$ & - & - & -- & - & -- & - \\
\hline & $05-13-97$ & - & -- & - & -- & -- & - \\
\hline \multirow[t]{18}{*}{24} & $10-15-96$ & -- & -- & - & -- & -- & -- \\
\hline & $12-03-96$ & $<.01$ & .06 & .030 & -- & - & $<.010$ \\
\hline & $02-11-97$ & -- & -- & -- & - & -- & - \\
\hline & $04-18-97$ & -- & -- & -- & - & -- & - \\
\hline & $05-08-97$ & -- & - & -- & -- & -- & -- \\
\hline & $05-21-97$ & -- & -- & $\cdots$ & -- & -- & - \\
\hline & $06-03-97$ & -- & -- & -- & -- & -- & -- \\
\hline & $06-11-97$ & -- & -- & - & -- & -- & - \\
\hline & $06-17-97$ & -- & -- & -- & -- & -- & - \\
\hline & $07-18-97$ & $<.01$ & $<.05$ & $<.015$ & -- & -. & $<.010$ \\
\hline & $09-23-97$ & $<.01$ & $<.05$ & $<.015$ & $<.2$ & .012 & $<.010$ \\
\hline & $02-09-98$ & $<.01$ & .10 & .039 & - & - & .016 \\
\hline & 04-29-98 & -- & -- & -- & -- & -- & -- \\
\hline & $05-05-98$ & -- & -- & -- & - & -- & -- \\
\hline & $09-23-98$ & -- & - & -- & $<.1$ & $<.05$ & - \\
\hline & $05-25-99$ & - & -- & -- & -- & -- & -- \\
\hline & $09-14-99$ & -- & -- & -- & - & -- & -- \\
\hline & \multicolumn{7}{|c|}{ CATARACT CREEK } \\
\hline \multirow[t]{2}{*}{26} & $10-18-96$ & -- & -- & -- & -- & -- & - \\
\hline & $09-26-97$ & -- & -- & - & - & -- & -- \\
\hline \multirow[t]{2}{*}{27} & $10-18-96$ & -- & -- & -- & -- & -- & -- \\
\hline & $09-26-97$ & -- & -- & -- & - & -- & -- \\
\hline \multirow[t]{3}{*}{28} & $05-28-97$ & - & -. & -- & -- & -- & -- \\
\hline & $09-26-97$ & - & -- & -- & - & -- & -- \\
\hline & $09-22-98$ & -- & -- & - & $<.1$ & $<.05$ & -- \\
\hline \multirow[t]{3}{*}{29} & $10-18-96$ & -- & -- & - & -- & -- & -- \\
\hline & $05-28-97$ & -- & - & -- & - & -- & -- \\
\hline & $09-22-98$ & -- & -- & -- & - & -- & - \\
\hline \multirow[t]{3}{*}{30} & $10-18-96$ & - & -- & - & -- & -- & -- \\
\hline & $05-28-97$ & - & - & -- & -- & -- & -- \\
\hline & $09-26-97$ & $\cdots$ & -- & -- & - & -- & -- \\
\hline \multirow[t]{2}{*}{31} & $05-28-97$ & -- & -- & -- & -- & -- & -- \\
\hline & $09-26-97$ & -- & -- & -- & -- & -- & - \\
\hline \multirow[t]{2}{*}{32} & $10-18-96$ & -- & -- & - & - & -- & -- \\
\hline & $05-20-97$ & -- & -- & - & - & -- & -- \\
\hline
\end{tabular}


Table 2. Water-quality data for streams in the Boulder River watershed, Montana, 1996-99 (Continued)

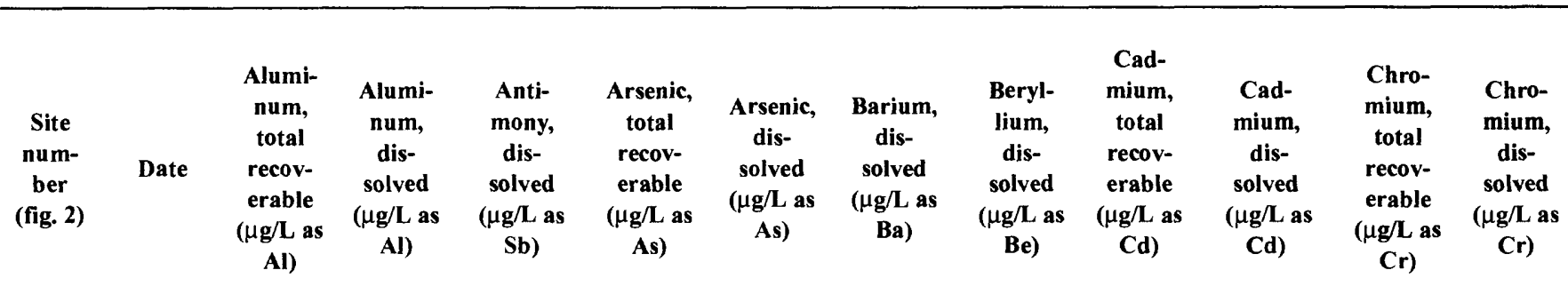

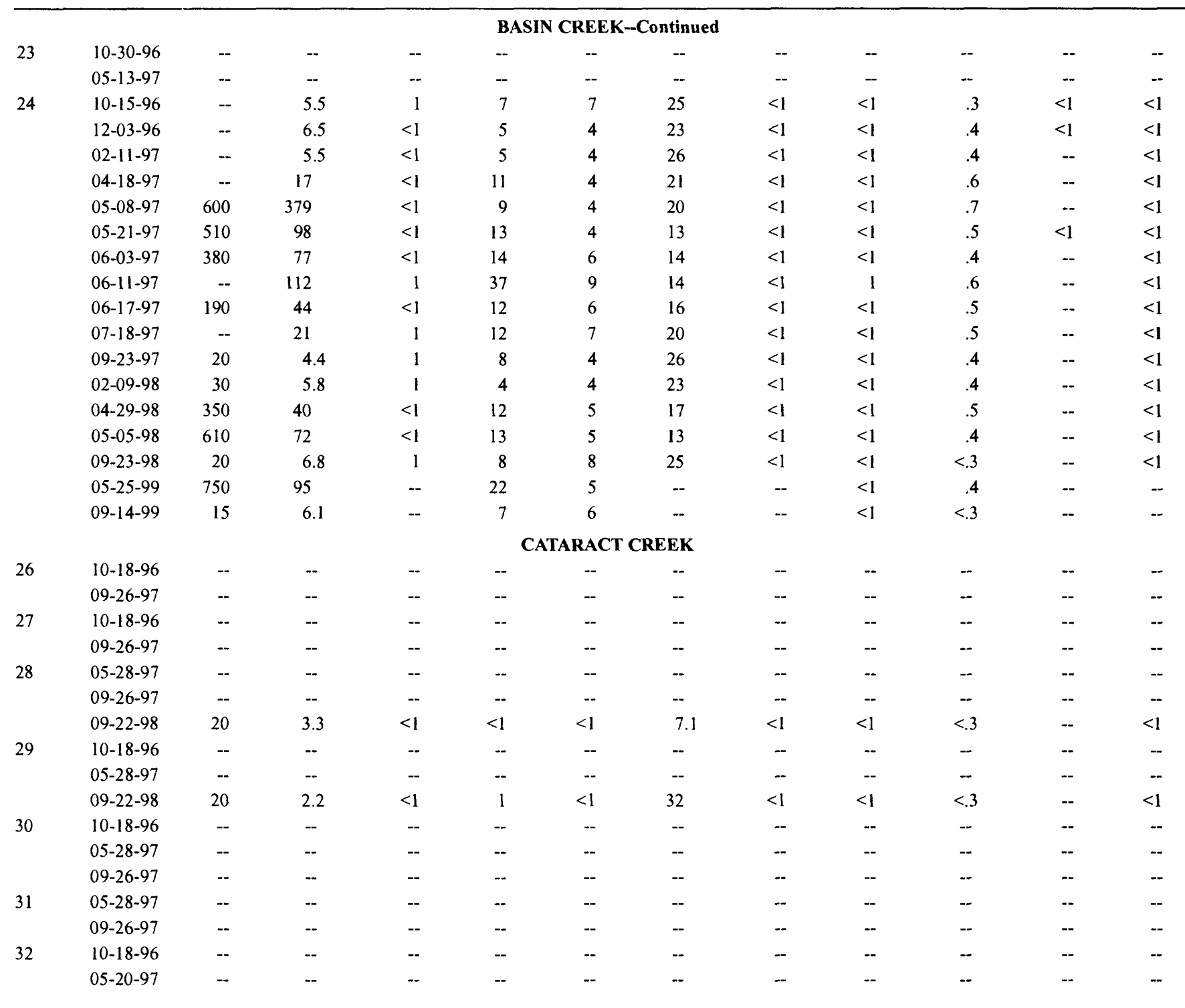


Table 2. Water-quality data for streams in the Boulder River watershed, Montana, 1996-99 (Continued)

\begin{tabular}{|c|c|c|c|c|c|c|c|c|c|c|c|}
\hline $\begin{array}{c}\text { Site } \\
\text { num- } \\
\text { ber } \\
\text { (fig. 2) }\end{array}$ & Date & $\begin{array}{c}\text { Cobalt, } \\
\text { dis- } \\
\text { solved } \\
(\mu \mathrm{g} / \mathrm{L} \text { as } \\
\text { Co })\end{array}$ & $\begin{array}{l}\text { Copper, } \\
\text { total recov- } \\
\text { erable } \\
(\mu \mathrm{g} / \mathrm{L} \text { as } \\
\text { Cu) }\end{array}$ & $\begin{array}{l}\text { Copper, } \\
\text { dis- } \\
\text { solved } \\
(\mu \mathrm{g} / \mathrm{L} \text { as } \\
\mathrm{Cu})\end{array}$ & $\begin{array}{c}\text { Iron, } \\
\text { total } \\
\text { recov- } \\
\text { erable } \\
(\mu \mathrm{g} / \mathrm{L} \text { as } \\
\mathrm{Fe})\end{array}$ & $\begin{array}{c}\text { Iron, } \\
\text { dis- } \\
\text { solved } \\
(\mu \mathrm{g} / \mathrm{L} \text { as } \\
\text { Fe })\end{array}$ & $\begin{array}{c}\text { Lead, } \\
\text { total } \\
\text { recov- } \\
\text { erable } \\
(\mu \mathrm{g} / \mathrm{L} \text { as } \\
\mathrm{Pb})\end{array}$ & $\begin{array}{c}\text { Lead, } \\
\text { dis- } \\
\text { solved } \\
(\mu \mathrm{g} / \mathrm{L} \text { as } \\
\text { Pb) }\end{array}$ & $\begin{array}{c}\text { Lithium, } \\
\text { dis- } \\
\text { solved } \\
(\mu \mathrm{g} / \mathrm{L} \text { as } \\
\mathrm{Li})\end{array}$ & $\begin{array}{c}\text { Manga- } \\
\text { nese, } \\
\text { total } \\
\text { recov- } \\
\text { erable } \\
\text { ( } \mu \text { g/L as } \\
\text { Mn) }\end{array}$ & $\begin{array}{c}\text { Manga- } \\
\text { nese, } \\
\text { dis- } \\
\text { solved } \\
\text { ( } \mu \mathrm{g} / \mathrm{L} \text { as } \\
\mathrm{Mn})\end{array}$ \\
\hline
\end{tabular}

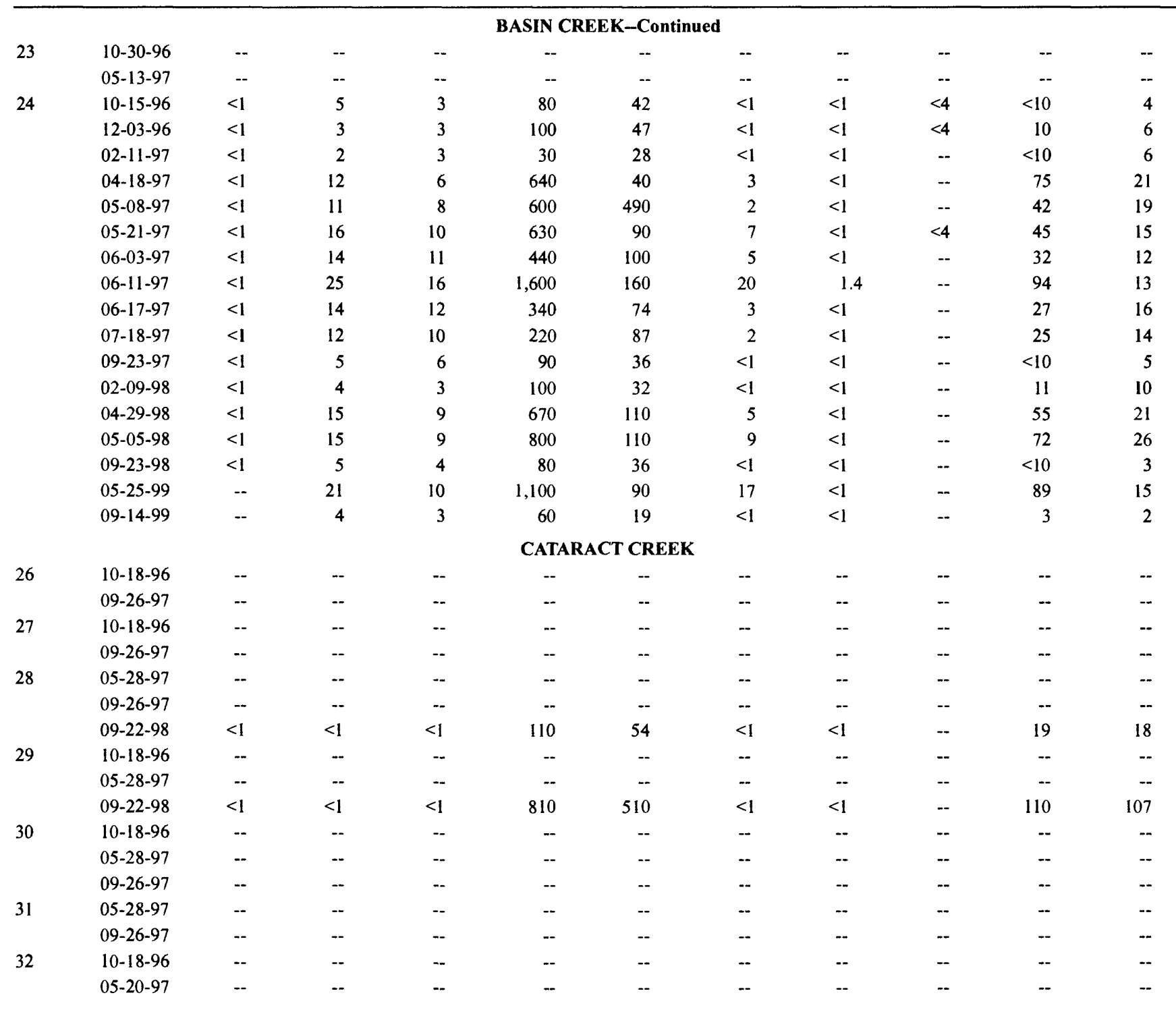


Table 2. Water-quality data for streams in the Boulder River watershed, Montana, 1996-99 (Continued)

\begin{tabular}{|c|c|c|c|c|c|c|c|c|c|c|c|}
\hline $\begin{array}{c}\text { Site } \\
\text { num- } \\
\text { ber } \\
\text { (fig. 2) }\end{array}$ & Date & $\begin{array}{c}\text { Mercury, } \\
\text { total } \\
\text { recov- } \\
\text { erable } \\
(\mu \mathrm{g} / \mathrm{L} \text { as } \\
\mathbf{H g})\end{array}$ & $\begin{array}{c}\text { Mercury, } \\
\text { dis- } \\
\text { solved } \\
(\mu \mathrm{g} / \mathrm{L} \text { as } \\
\mathrm{Hg})\end{array}$ & $\begin{array}{c}\text { Molyb- } \\
\text { denum, } \\
\text { dis- } \\
\text { solved } \\
(\mu \mathrm{g} / \mathrm{L} \text { as } \\
\text { Mo })\end{array}$ & $\begin{array}{c}\text { Nickel, } \\
\text { total } \\
\text { recov- } \\
\text { erable } \\
(\mu \mathrm{g} / \mathrm{L} \text { as } \\
\mathrm{Ni})\end{array}$ & $\begin{array}{l}\text { Nickel, } \\
\text { dis- } \\
\text { solved } \\
(\mu \mathrm{g} / \mathrm{L} \text { as } \\
\mathrm{Ni})\end{array}$ & $\begin{array}{c}\text { Silver, } \\
\text { total } \\
\text { recov- } \\
\text { erable } \\
(\mu \mathrm{g} / \mathrm{L} \text { as } \\
\mathbf{A g})\end{array}$ & $\begin{array}{l}\text { Silver, } \\
\text { dis- } \\
\text { solved } \\
(\mu \mathrm{g} / \mathrm{L} \text { as } \\
\mathrm{Ag})\end{array}$ & $\begin{array}{c}\text { Uranium, } \\
\text { dis- } \\
\text { solved } \\
(\mu \mathrm{g} / \mathrm{L} \text { as } \\
\text { U) }\end{array}$ & $\begin{array}{c}\text { Zinc, } \\
\text { total } \\
\text { recov- } \\
\text { erable } \\
(\mu \mathrm{g} / \mathrm{L} \text { as } \\
\mathrm{Zn})\end{array}$ & $\begin{array}{c}\text { Zinc, } \\
\text { dis- } \\
\text { solved } \\
(\mu \mathrm{g} / \mathrm{L} \text { as } \\
\mathrm{Zn})\end{array}$ \\
\hline
\end{tabular}

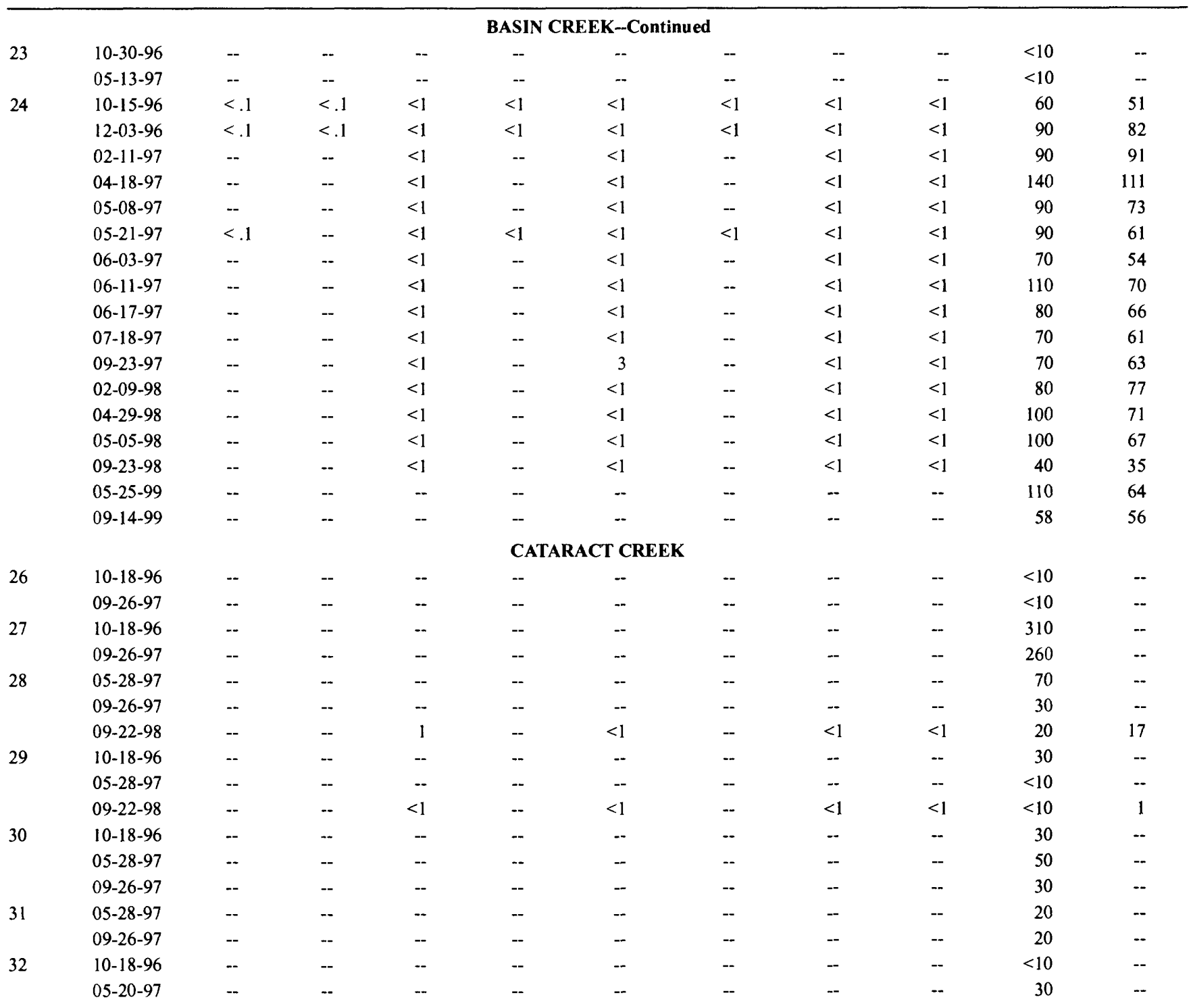


Table 2. Water-quality data for streams in the Boulder River watershed, Montana, 1996-99 (Continued)

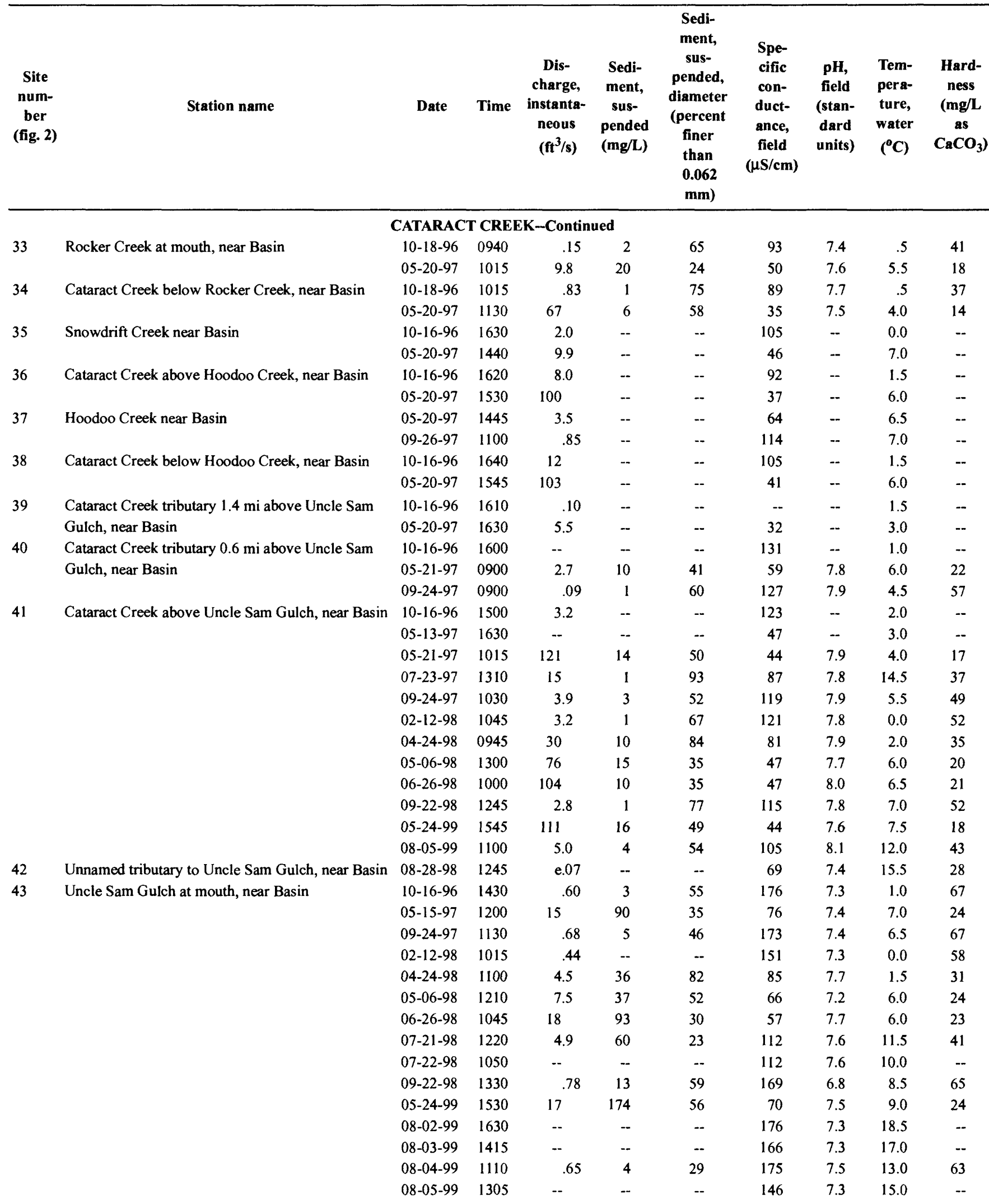


Table 2. Water-quality data for streams in the Boulder River watershed, Montana, 1996-99 (Continued)

\begin{tabular}{|c|c|c|c|c|c|c|c|c|c|c|}
\hline $\begin{array}{c}\text { Site } \\
\text { num- } \\
\text { ber } \\
\text { (fig. 2) }\end{array}$ & Date & $\begin{array}{c}\text { Cal- } \\
\text { cium, } \\
\text { dissolved } \\
(\mathrm{mg} / \mathrm{L} \\
\text { as Ca) }\end{array}$ & $\begin{array}{c}\text { Magne- } \\
\text { sium, } \\
\text { dissolved } \\
\text { (mg/L } \\
\text { as Mg) }\end{array}$ & $\begin{array}{c}\text { Sodium, } \\
\text { dissolved } \\
(\mathrm{mg} / \mathrm{L} \\
\text { as } \mathrm{Na})\end{array}$ & $\begin{array}{c}\text { Potas- } \\
\text { sium, } \\
\text { dissolved } \\
(\mathrm{mg} / \mathbf{L} \\
\text { as K) }\end{array}$ & $\begin{array}{c}\mathrm{ANC}, \\
\text { lab } \\
(\mathrm{mg} / \mathrm{L} \\
\left.\text { as } \mathrm{CaCO}_{3}\right)\end{array}$ & $\begin{array}{c}\text { Sulfate, } \\
\text { dissolved } \\
(\mathrm{mg} / \mathrm{L} \\
\left.\text { as } \mathrm{SO}_{4}\right)\end{array}$ & $\begin{array}{c}\text { Chloride, } \\
\text { dissolved } \\
\text { (mg/L } \\
\text { as } \mathrm{Cl})\end{array}$ & $\begin{array}{c}\text { Fluoride, } \\
\text { dissolved } \\
\text { (mg/L } \\
\text { as F) }\end{array}$ & $\begin{array}{c}\text { Silica, } \\
\text { dissolved } \\
(\mathrm{mg} / \mathrm{L} \\
\left.\text { as } \mathrm{SiO}_{2}\right)\end{array}$ \\
\hline
\end{tabular}

\begin{tabular}{|c|c|c|c|c|c|c|c|c|c|c|c|}
\hline \multicolumn{12}{|c|}{ CATARACT CREEK-Continued } \\
\hline \multirow[t]{2}{*}{33} & $10-18-96$ & 12 & 2.6 & 2.8 & .90 & 35 & 11 & .40 & $<.10$ & 14 & 65 \\
\hline & $05-20-97$ & 5.4 & 1.1 & 1.5 & .64 & 12 & 8.7 & .25 & $<.10$ & 11 & 37 \\
\hline \multirow[t]{2}{*}{34} & $10-18-96$ & 11 & 2.4 & 2.6 & .90 & 36 & 7.4 & 1.3 & $<.10$ & 13 & 60 \\
\hline & $05-20-97$ & 4.3 & .81 & 1.2 & .63 & 12 & 3.7 & .24 & $<.10$ & 8.9 & 27 \\
\hline \multirow[t]{2}{*}{35} & $10-16-96$ & -- & -- & -- & -- & -- & -- & - & -- & -- & -- \\
\hline & $05-20-97$ & -- & -- & -- & -- & -- & -- & -. & -- & -- & -- \\
\hline \multirow[t]{2}{*}{36} & $10-16-96$ & -- & -- & -- & -- & - & -- & -- & -- & -- & -- \\
\hline & $05-20-97$ & -- & -- & - & -- & - & -- & -- & -- & -- & -- \\
\hline \multirow[t]{2}{*}{37} & $05-20-97$ & -- & -- & -- & - & - & -- & -- & -- & -- & -- \\
\hline & $09-26-97$ & -- & -- & -- & -- & -- & -- & -- & -- & -- & -- \\
\hline \multirow[t]{2}{*}{38} & $10-16-96$ & -- & -- & $\ldots$ & -- & -- & -- & - & -- & -- & -- \\
\hline & $05-20-97$ & -- & -- & -- & -- & -- & -- & -- & -- & -- & - \\
\hline \multirow[t]{2}{*}{39} & $10-16-96$ & -- & -- & -- & -- & -- & -- & -- & -- & -- & - \\
\hline & $05-20-97$ & -- & -- & -- & -- & - & -- & -- & - & -- & - \\
\hline \multirow[t]{3}{*}{40} & $10-16-96$ & -- & -- & -- & -- & -- & -- & -- & -- & -- & -- \\
\hline & $05-21-97$ & 6.6 & 1.2 & 2.0 & .65 & 14 & 11 & .43 & $<.10$ & 13 & 44 \\
\hline & 09-24-97 & 18 & 3.2 & 3.4 & 1.0 & 43 & 19 & .50 & $<.10$ & 18 & 89 \\
\hline \multirow[t]{12}{*}{41} & $10-16-96$ & -- & -- & - & -- & -- & -- & - & -- & -- & - \\
\hline & 05-13-97 & -- & -- & -- & -- & -- & -- & -- & -- & -- & -- \\
\hline & $05-21-97$ & 5.0 & 1.0 & 1.5 & .60 & 14 & 5.3 & .28 & $<.10$ & 9.9 & 32 \\
\hline & $07-23-97$ & 11 & 2.0 & 2.5 & .89 & 36 & 7.6 & .25 & $<.10$ & 14 & 60 \\
\hline & $09-24-97$ & 15 & 2.6 & 3.1 & 1.1 & 47 & 13 & .49 & $<.10$ & 15 & 79 \\
\hline & $02-12-98$ & 16 & 3.0 & 3.2 & 1.0 & 44 & 14 & 1.5 & $<.10$ & 15 & 80 \\
\hline & $04-24-98$ & 11 & 2.0 & 2.1 & 1.9 & 33 & 7.1 & .64 & $<.10$ & 10 & 55 \\
\hline & $05-06-98$ & 6.1 & 1.1 & 1.5 & .87 & 18 & 4.4 & .31 & $<.10$ & 9.3 & 35 \\
\hline & $06-26-98$ & 6.6 & 1.2 & 1.9 & .53 & 19 & 5.0 & .18 & $<.10$ & 11 & 38 \\
\hline & 09-22-98 & 16 & 2.9 & 3.8 & 1.0 & 48 & 13 & .49 & $<.10$ & 15 & 81 \\
\hline & $05-24-99$ & 5.3 & 1.0 & 1.5 & .71 & 15 & 4.7 & .22 & -- & -- & -- \\
\hline & 08-05-99 & 13 & 2.5 & 3.1 & 1.1 & 41 & 9.4 & .39 & -- & -- & -- \\
\hline 42 & 08-28-98 & 8.4 & 1.7 & 2.6 & .72 & 27 & 7.4 & $<.1$ & $<.10$ & 13 & 43 \\
\hline \multirow[t]{15}{*}{43} & $10-16-96$ & 20 & 4.1 & 3.3 & 1.0 & 29 & 52 & .70 & $<.10$ & 17 & 120 \\
\hline & $05-15-97$ & 7.2 & 1.4 & 1.9 & .71 & 10 & 18 & .28 & $<.10$ & 12 & 49 \\
\hline & $09-24-97$ & 20 & 4.2 & 3.4 & 1.0 & 28 & 51 & .46 & $<.10$ & 17 & 119 \\
\hline & $02-12-98$ & 17 & 3.7 & 3.3 & .80 & 28 & 41 & .63 & $<.10$ & 17 & 103 \\
\hline & $04-24-98$ & 9.5 & 1.9 & 2.4 & .83 & 17 & 20 & .36 & $<.10$ & 13 & 59 \\
\hline & $05-06-98$ & 7.2 & 1.5 & 2.0 & .70 & 11 & 16 & .42 & $<.10$ & 12 & 48 \\
\hline & $06-26-98$ & 7.0 & 1.3 & 2.1 & .63 & 14 & 13 & .20 & $<.10$ & 13 & 47 \\
\hline & $07-21-98$ & 12 & 2.4 & 2.8 & .79 & 22 & 26 & .39 & $<.10$ & 16 & 76 \\
\hline & $07-22-98$ & -- & -- & -- & -- & -- & -- & -- & -- & -- & -- \\
\hline & $09-22-98$ & 19 & 4.1 & 3.5 & 1.0 & 28 & 54 & .47 & $<.1$ & 18 & 122 \\
\hline & $05-24-99$ & 7.0 & 1.5 & 2.0 & .69 & 10 & 18 & .25 & -- & -- & \\
\hline & $08-02-99$ & -- & -- & -- & -- & -- & -- & -- & -- & -- & - \\
\hline & 08-03-99 & -- & -- & -- & -- & -- & -- & -- & - & -- & -- \\
\hline & 08-04-99 & 18 & 4.0 & 3.3 & 1.1 & 21 & 57 & .35 & -- & -- & - \\
\hline & 08-05-99 & -- & - & -- & -- & -- & -- & -- & -- & -- & -- \\
\hline
\end{tabular}


Table 2. Water-quality data for streams in the Boulder River watershed, Montana, 1996-99 (Continued)

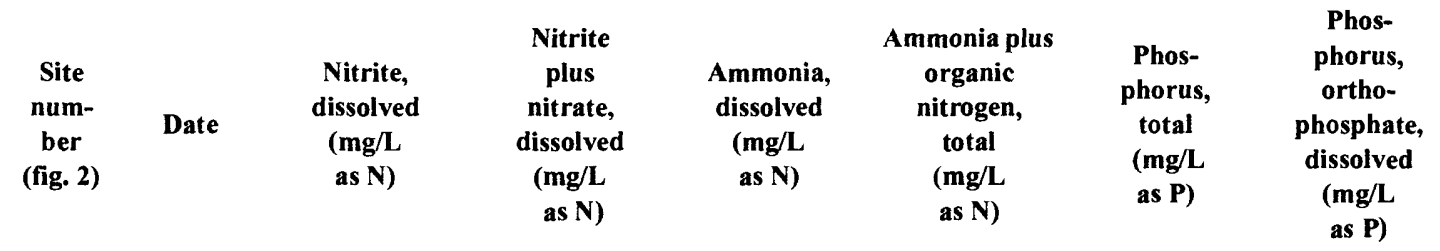

\begin{tabular}{|c|c|c|c|c|c|c|c|}
\hline & & & CAT & REEK- & & & \\
\hline 33 & $10-18-96$ & - & - & -- & - & -- & $\ldots$ \\
\hline & $05-20-97$ & -- & -- & -- & -- & -- & -- \\
\hline 34 & $10-18-96$ & -- & -- & -- & -- & -- & -- \\
\hline & $05-20-97$ & - & - & -- & -- & -- & - \\
\hline 35 & $10-16-96$ & -- & -- & -- & - & -- & -- \\
\hline & $05-20-97$ & - & -- & -- & - & -- & -- \\
\hline 36 & $10-16-96$ & -- & -- & -- & -- & - & -- \\
\hline & $05-20-97$ & - & -- & - & - & - & -- \\
\hline 37 & $05-20-97$ & - & -- & - & - & -. & $\ldots$ \\
\hline & $09-26-97$ & - & -- & -- & -- & -- & -- \\
\hline 38 & $10-16-96$ & -- & -- & -- & -- & -- & -- \\
\hline & $05-20-97$ & - & -. & - & - & - & -. \\
\hline 39 & $10-16-96$ & -- & -- & -- & -- & - & - \\
\hline & $05-20-97$ & -- & -- & -- & - & -- & -- \\
\hline 40 & $10-16-96$ & - & -- & -- & -- & -- & - \\
\hline & $05-21-97$ & - & - & -.- & - & - & -. \\
\hline & $09-24-97$ & $<.01$ & $<.05$ & $<.015$ & $<.2$ & $<.010$ & $<.010$ \\
\hline 41 & $10-16-96$ & -- & -- & -. & -. & -. & - \\
\hline & $05-13-97$ & -- & - & -. & -- & -- & -- \\
\hline & $05-21-97$ & -- & - & -- & - & -- & -- \\
\hline & $07-23-97$ & $<.01$ & $<.05$ & $<.015$ & -. & -. & $<.010$ \\
\hline & $09-24-97$ & $<.01$ & $<.05$ & $<.015$ & $<.2$ & $<.010$ & $<.010$ \\
\hline & $02-12-98$ & $<.01$ & .10 & .038 & -- & -- & .013 \\
\hline & $04-24-98$ & -- & -- & - & - & -- & - \\
\hline & $05-06-98$ & -- & -- & -- & -- & -- & - \\
\hline & $06-26-98$ & -- & -- & -- & -- & -- & -- \\
\hline & $09-22-98$ & - & -- & -. & -- & -- & -- \\
\hline & $05-24-99$ & -- & -- & - & -- & -- & -- \\
\hline & $08-05-99$ & -- & - & -- & -- & -- & -- \\
\hline 42 & $08-28-98$ & -- & -- & -. & -. & - & -- \\
\hline 43 & $10-16-96$ & -- & -- & -- & - & -- & -- \\
\hline & $05-15-97$ & - & - & -- & -- & -- & -. \\
\hline & $09-24-97$ & $<.01$ & $<.05$ & $<.015$ & $<.2$ & $<.010$ & $<.010$ \\
\hline & $02-12-98$ & $<.01$ & .10 & .031 & -- & -- & .012 \\
\hline & $04-24-98$ & -- & -- & -- & - & -- & -- \\
\hline & $05-06-98$ & -- & -- & - & $\ldots$ & -- & - \\
\hline & $06-26-98$ & - & - & - & -- & - & - \\
\hline & $07-21-98$ & $<.01$ & $<.05$ & .037 & - & - & $<.010$ \\
\hline & $07-22-98$ & -- & -- & -- & -- & - & -- \\
\hline & $09-22-98$ & -- & - & - & -- & -- & -- \\
\hline & $05-24-99$ & -- & -- & -- & -- & -- & -- \\
\hline & $08-02-99$ & -- & -- & -- & -- & -- & -- \\
\hline & 08-03-99 & -- & - & -- & -- & - & -- \\
\hline & $08-04-99$ & -- & -- & -- & -- & -- & - \\
\hline & $08-05-99$ & -- & -- & .- & -- & -- & -- \\
\hline
\end{tabular}


Table 2. Water-quality data for streams in the Boulder River watershed, Montana, 1996-99 (Continued)

\begin{tabular}{|c|c|c|c|c|c|c|c|c|c|c|c|c|}
\hline $\begin{array}{c}\text { Site } \\
\text { num- } \\
\text { ber } \\
\text { (fig. 2) }\end{array}$ & Date & $\begin{array}{l}\text { Alumi- } \\
\text { num, } \\
\text { total } \\
\text { recov- } \\
\text { erable } \\
(\mu \mathrm{g} / \mathrm{L} \text { as } \\
\mathrm{Al})\end{array}$ & $\begin{array}{l}\text { Alumi- } \\
\text { num, } \\
\text { dis- } \\
\text { solved } \\
(\mu \mathrm{g} / \mathrm{L} \text { as } \\
\text { Al) }\end{array}$ & $\begin{array}{c}\text { Anti- } \\
\text { mony, } \\
\text { dis- } \\
\text { solved } \\
(\mu \mathrm{g} / \mathrm{L} \text { as } \\
\text { Sb) }\end{array}$ & $\begin{array}{c}\text { Arsenic, } \\
\text { total } \\
\text { recov- } \\
\text { erable } \\
(\mu \mathrm{g} / \mathrm{L} \text { as } \\
\text { As) }\end{array}$ & $\begin{array}{c}\text { Arsenic, } \\
\text { dis- } \\
\text { solved } \\
(\mu \mathrm{g} / \mathrm{L} \text { as } \\
\text { As) }\end{array}$ & $\begin{array}{c}\text { Barium, } \\
\text { dis- } \\
\text { solved } \\
(\mu \mathrm{g} / \mathrm{L} \text { as } \\
\text { Ba) }\end{array}$ & $\begin{array}{c}\text { Beryl- } \\
\text { lium, } \\
\text { dis- } \\
\text { solved } \\
(\mu \mathrm{g} / \mathrm{L} \text { as } \\
\text { Be) }\end{array}$ & $\begin{array}{c}\text { Cad- } \\
\text { mium, } \\
\text { total } \\
\text { recov- } \\
\text { erable } \\
(\mu \mathrm{g} / \mathrm{L} \text { as } \\
\text { Cd })\end{array}$ & $\begin{array}{l}\text { Cad- } \\
\text { mium, } \\
\text { dis- } \\
\text { solved } \\
\text { ( } \mu \text { g/L as } \\
\text { Cd) }\end{array}$ & $\begin{array}{c}\text { Chro- } \\
\text { mium, } \\
\text { total } \\
\text { recov- } \\
\text { erable } \\
(\mu \mathrm{g} / \mathrm{L} \text { as } \\
\text { Cr) }\end{array}$ & $\begin{array}{c}\text { Chro- } \\
\text { mium, } \\
\text { dis- } \\
\text { solved } \\
\text { ( } \mu \mathrm{g} / \mathrm{L} \text { as } \\
\mathrm{Cr})\end{array}$ \\
\hline
\end{tabular}

\begin{tabular}{|c|c|c|c|c|c|c|c|c|c|c|c|c|}
\hline \multicolumn{13}{|c|}{ CATARACT CREEK-Continued } \\
\hline \multirow[t]{2}{*}{33} & $10-18-96$ & - & 3.5 & $<1$ & 3 & 2 & 9.0 & $<1$ & $<1$ & $<.1$ & $<1$ & $<1$ \\
\hline & $05-20-97$ & 460 & 92 & $<1$ & 9 & 2 & 7.8 & $<1$ & $<1$ & .3 & $<1$ & $<1$ \\
\hline \multirow[t]{2}{*}{34} & $10-18-96$ & -- & 4.5 & $<1$ & 1 & 1 & 13 & $<1$ & $<1$ & $<.1$ & $<1$ & $<1$ \\
\hline & $05-20-97$ & 260 & 111 & $<1$ & 3 & 1 & 8.9 & $<1$ & $<1$ & .1 & $<1$ & $<1$ \\
\hline \multirow[t]{2}{*}{35} & $10-16-96$ & -- & -- & -- & -- & -- & -- & -- & -- & -- & -- & -- \\
\hline & $05-20-97$ & -- & -- & -- & -- & -- & -- & -- & -- & -- & -- & - \\
\hline \multirow[t]{2}{*}{37} & $05-20-97$ & - & - & - & -. & -- & -- & -- & - & -- & -- & - \\
\hline & $09-26-97$ & -- & -- & -- & -- & -- & -- & -- & -- & -- & -- & -- \\
\hline \multirow[t]{2}{*}{38} & $10-16-96$ & -- & -- & -- & -- & -- & -- & -- & -- & -- & -- & - \\
\hline & $05-20-97$ & -- & -- & -. & - & -- & -- & -- & -- & -- & - & - \\
\hline 39 & $10-16-96$ & -- & -- & -- & -- & -- & -- & -- & -- & -- & -- & - \\
\hline \multirow[t]{11}{*}{41} & $10-16-96$ & -- & -- & - & -- & -- & -- & -- & -- & - & - & -- \\
\hline & $05-13-97$ & -- & -- & -- & -- & -- & -- & -- & -- & -- & -. & - \\
\hline & $05-21-97$ & 310 & 88 & $<1$ & 4 & 2 & 10 & $<1$ & $<1$ & .2 & - & $<1$ \\
\hline & $07-23-97$ & 30 & 15 & $<1$ & 3 & 3 & 16 & $<1$ & $<1$ & .4 & - & $<1$ \\
\hline & $09-24-97$ & $<10$ & 3.0 & $<1$ & 3 & 2 & 17 & $<1$ & $<1$ & .3 & - & $<1$ \\
\hline & $02-12-98$ & $<10$ & 9.0 & $<1$ & 2 & 2 & 15 & $<1$ & $<1$ & .3 & -. & $<1$ \\
\hline & $04-24-98$ & 240 & 22 & $<1$ & 4 & 2 & 15 & $<1$ & $<1$ & $<.3$ & -- & $<1$ \\
\hline & $05-06-98$ & 290 & 46 & $<1$ & 3 & 2 & 10 & $<1$ & $<1$ & $<.3$ & - & $<1$ \\
\hline & $06-26-98$ & 150 & 48 & $<1$ & 2 & 5 & 10 & $<1$ & $<1$ & $<.3$ & - & $<1$ \\
\hline & $09-22-98$ & 10 & 3.0 & $<1$ & 3 & 3 & 16 & $<1$ & $<1$ & $<.3$ & - & $<1$ \\
\hline & $05-24-99$ & 375 & 56 & -- & 6 & 3 & -- & -- & $<1$ & $<.3$ & - & -- \\
\hline \multirow{10}{*}{43} & $05-06-98$ & 660 & 63 & 2 & 110 & 6 & 13 & $<1$ & 11 & 9.8 & -- & $<1$ \\
\hline & $06-26-98$ & 880 & 61 & 2 & 170 & 2 & 12 & $<1$ & 11 & 7.8 & -- & $<1$ \\
\hline & $07-21-98$ & 410 & 55 & 2 & 48 & 3 & 20 & $<1$ & 23 & 22 & -- & $<1$ \\
\hline & $07-22-98$ & - & -- & -- & -- & -- & -- & -- & -- & 22 & - & - \\
\hline & $09-22-98$ & 560 & 16 & 1 & 7 & 1 & 28 & $<1$ & 52 & 54 & -- & $<1$ \\
\hline & $05-24-99$ & 1,890 & 45 & -- & 760 & 5 & $-\cdot$ & -- & 20 & 11 & -- & -- \\
\hline & $08-02-99$ & -- & -- & -- & -- & -- & -- & -- & -- & 75 & - & - \\
\hline & $08-03-99$ & -- & -- & -- & -- & -- & - & -- & -- & 65 & -- & -- \\
\hline & $08-04-99$ & 390 & 18 & -- & 12 & 1 & -- & -- & 61 & 59 & - & - \\
\hline & $08-05-99$ & -- & -. & -- & -- & -- & -- & -- & -- & 39 & -- & - \\
\hline
\end{tabular}


Table 2. Water-quality data for streams in the Boulder River watershed, Montana, 1996-99 (Continued)

\begin{tabular}{|c|c|c|c|c|c|c|c|c|c|c|c|}
\hline $\begin{array}{c}\text { Site } \\
\text { num- } \\
\text { ber } \\
\text { (fig. 2) }\end{array}$ & Date & $\begin{array}{l}\text { Cobalt, } \\
\text { dis- } \\
\text { solved } \\
(\mu \mathrm{g} / \mathrm{L} \text { as } \\
\text { Co) }\end{array}$ & $\begin{array}{l}\text { Copper, } \\
\text { total recov- } \\
\text { erable } \\
(\mu \mathrm{g} / \mathrm{L} \text { as } \\
\text { Cu })\end{array}$ & $\begin{array}{l}\text { Copper, } \\
\text { dis- } \\
\text { solved } \\
(\mu \mathrm{g} / \mathrm{L} \text { as } \\
\text { Cu) }\end{array}$ & $\begin{array}{c}\text { Iron, } \\
\text { total } \\
\text { recov- } \\
\text { erable } \\
(\mu \mathrm{g} / \mathrm{L} \text { as } \\
\text { Fe })\end{array}$ & $\begin{array}{c}\text { Iron, } \\
\text { dis- } \\
\text { solved } \\
(\mu \mathrm{g} / \mathrm{L} \text { as } \\
\text { Fe) }\end{array}$ & $\begin{array}{c}\text { Lead, } \\
\text { total } \\
\text { recov- } \\
\text { erable } \\
(\mu \mathrm{g} / \mathrm{L} \text { as } \\
\text { Pb) }\end{array}$ & $\begin{array}{c}\text { Lead, } \\
\text { dis- } \\
\text { solved } \\
(\mu \mathrm{g} / \mathrm{L} \text { as } \\
\mathrm{Pb})\end{array}$ & $\begin{array}{c}\text { Lithium, } \\
\text { dis- } \\
\text { solved } \\
(\mu \mathrm{g} / \mathrm{L} \text { as } \\
\mathrm{Li})\end{array}$ & $\begin{array}{l}\text { Manga- } \\
\text { nese, } \\
\text { total } \\
\text { recov- } \\
\text { erable } \\
(\mu \mathrm{g} / \mathrm{L} \text { as } \\
\text { Mn) }\end{array}$ & $\begin{array}{c}\text { Manga- } \\
\text { nese, } \\
\text { dis- } \\
\text { solved } \\
(\mu \mathrm{g} / \mathrm{L} \text { as } \\
\text { Mn) }\end{array}$ \\
\hline
\end{tabular}

\begin{tabular}{|c|c|c|c|c|c|c|c|c|c|c|c|}
\hline \multicolumn{12}{|c|}{ CATARACT CREEK-Continued } \\
\hline \multirow[t]{2}{*}{33} & $10-18-96$ & $<1$ & 7 & 5 & 490 & 300 & $<1$ & $<1$ & $<4$ & 80 & 22 \\
\hline & $05-20-97$ & 1 & 44 & 35 & 670 & 130 & 2 & $<1$ & $<4$ & 56 & 47 \\
\hline \multirow[t]{2}{*}{34} & $10-18-96$ & $<1$ & 2 & 2 & 110 & 77 & $<1$ & $<1$ & $<4$ & $<10$ & 4 \\
\hline & $05-20-97$ & $<1$ & 8 & 7 & 360 & 180 & $<1$ & $<1$ & $<4$ & 19 & 11 \\
\hline \multirow[t]{2}{*}{35} & $10-16-96$ & -- & -- & -- & -- & -- & -- & -- & -- & -- & -- \\
\hline & $05-20-97$ & -- & -- & -- & -- & -- & - & -- & -- & -- & - \\
\hline \multirow[t]{2}{*}{36} & $10-16-96$ & -- & -- & -- & -- & -- & - & - & -- & - & - \\
\hline & $05-20-97$ & -- & -- & -- & -- & - & - & -- & - & - & - \\
\hline \multirow[t]{2}{*}{37} & $05-20-97$ & -- & -- & -- & -- & -- & -- & -- & -- & -- & - \\
\hline & $09-26-97$ & -- & -- & -- & -- & -- & -- & -- & - & -- & - \\
\hline \multirow[t]{2}{*}{38} & $10-16-96$ & - & -- & -- & -- & -. & -- & -- & -- & -- & -- \\
\hline & $05-20-97$ & -- & -- & -- & -- & -- & -- & -- & - & -- & -- \\
\hline \multirow[t]{2}{*}{39} & $10-16-96$ & -- & -- & -- & -- & -- & -- & -- & -- & - & - \\
\hline & $05-20-97$ & -- & -- & -- & -. & - & -- & -- & - & - & - \\
\hline \multirow[t]{3}{*}{40} & $10-16-96$ & -- & -- & -- & -- & -- & -- & -- & -- & -- & - \\
\hline & $05-21-97$ & $<1$ & 7 & 6 & 200 & 46 & 1 & $<1$ & -- & $<10$ & $<1$ \\
\hline & $09-24-97$ & $<1$ & $<1$ & 1 & 50 & 15 & $<1$ & $<1$ & -- & $<10$ & $<1$ \\
\hline \multirow[t]{12}{*}{41} & $10-16-96$ & -- & -- & -- & -- & -- & -- & -- & - & -- & -. \\
\hline & $05-13-97$ & - & -- & -- & -- & -- & -- & -- & -- & -- & -- \\
\hline & $05-21-97$ & $<1$ & 9 & 9 & 410 & 130 & 4 & $<1$ & -- & 26 & 8 \\
\hline & $07-23-97$ & $<1$ & 6 & 6 & 200 & 110 & $<1$ & $<1$ & -- & 10 & 6 \\
\hline & $09-24-97$ & $<1$ & 5 & 5 & 120 & 74 & $<1$ & $<1$ & -- & 12 & 6 \\
\hline & $02-12-98$ & $<1$ & 6 & 5 & 90 & 49 & $<1$ & $<1$ & -- & $<10$ & 4 \\
\hline & $04-24-98$ & $<1$ & 13 & 9 & 850 & 300 & 5 & $<1$ & -- & 74 & 16 \\
\hline & $05-06-98$ & $<1$ & 8 & 7 & 600 & 200 & 3 & $<1$ & -- & 38 & 16 \\
\hline & $06-26-98$ & $<1$ & 7 & 7 & 210 & 90 & 1 & $<1$ & -- & 13 & 5 \\
\hline & $09-22-98$ & $<1$ & 4 & 4 & 80 & 46 & $<1$ & $<1$ & -- & $<10$ & 3 \\
\hline & $05-24-99$ & -- & 11 & 7 & 550 & 87 & 4 & $<1$ & -- & 44 & 6 \\
\hline & $08-05-99$ & -- & 4 & 4 & 29 & 25 & $<1$ & $<1$ & -- & e2 & 3 \\
\hline 42 & $09-28-98$ & $<1$ & -- & 7 & -- & 130 & -- & 1.5 & -- & -- & 38 \\
\hline \multirow[t]{15}{*}{43} & $10-16-96$ & 11 & 530 & 226 & 200 & 7 & 10 & $<1$ & 5 & 640 & 597 \\
\hline & $05-15-97$ & 3 & 500 & 187 & 3,100 & 250 & 56 & 2 & $<4$ & 260 & 147 \\
\hline & $09-24-97$ & 13 & 470 & 200 & 350 & 4 & 13 & $<1$ & -- & 750 & 728 \\
\hline & $02-12-98$ & 5 & 190 & 127 & 70 & $<10$ & 4 & $<1$ & -- & 370 & 376 \\
\hline & $04-24-98$ & 2 & 460 & 134 & 2,200 & 68 & 50 & 1 & -- & 250 & 205 \\
\hline & $05-06-98$ & 3 & 340 & 166 & 1,900 & 120 & 41 & $<1$ & -- & 240 & 163 \\
\hline & $06-26-98$ & 2 & 340 & 136 & 2,900 & 99 & 72 & $<1$ & - & 320 & 111 \\
\hline & $07-21-98$ & 6 & 400 & 84 & 790 & 14 & 9 & $<1$ & -- & 310 & 323 \\
\hline & $07-22-98$ & - & -- & 84 & -- & -- & -- & -- & -- & -- & -- \\
\hline & $09-22-98$ & 13 & 530 & 231 & 230 & $<10$ & 11 & $<1$ & -- & 740 & 733 \\
\hline & $05-24-99$ & -- & 800 & 150 & 6,100 & 83 & 150 & $<1$ & -- & 490 & 154 \\
\hline & 08-02-99 & -- & -- & 377 & -- & - & -- & -- & -- & -- & -- \\
\hline & 08-03-99 & -- & -- & 274 & -- & - & -- & -- & -- & -- & - \\
\hline & 08-04-99 & -. & 440 & 206 & 230 & $<10$ & 6 & $<1$ & -- & 770 & 795 \\
\hline & $08-05-99$ & -- & -- & 117 & -- & - & -- & -- & -- & -- & - \\
\hline
\end{tabular}


Table 2. Water-quality data for streams in the Boulder River watershed, Montana, 1996-99 (Continued)

\begin{tabular}{|c|c|c|c|c|c|c|c|c|c|c|c|}
\hline $\begin{array}{c}\text { Site } \\
\text { num- } \\
\text { ber } \\
\text { (fig. 2) }\end{array}$ & Date & $\begin{array}{c}\text { Mercury, } \\
\text { total } \\
\text { recov- } \\
\text { erable } \\
(\mu \mathrm{g} / \mathrm{L} \text { as } \\
\mathrm{Hg})\end{array}$ & $\begin{array}{c}\text { Mercury, } \\
\text { dis- } \\
\text { solved } \\
(\mu \mathrm{g} / \mathrm{L} \text { as } \\
\mathrm{Hg})\end{array}$ & $\begin{array}{c}\text { Molyb- } \\
\text { denum, } \\
\text { dis- } \\
\text { solved } \\
(\mu \mathrm{g} / \mathrm{L} \text { as } \\
\text { Mo) }\end{array}$ & $\begin{array}{c}\text { Nickel, } \\
\text { total } \\
\text { recov- } \\
\text { erable } \\
(\mu \mathrm{g} / \mathrm{L} \text { as } \\
\mathrm{Ni})\end{array}$ & $\begin{array}{c}\text { Nickel, } \\
\text { dis- } \\
\text { solved } \\
(\mu \mathrm{g} / \mathrm{L} \text { as } \\
\mathrm{Ni})\end{array}$ & $\begin{array}{l}\text { Silver, } \\
\text { total } \\
\text { recov- } \\
\text { erable } \\
(\mu \mathrm{g} / \mathrm{L} \text { as } \\
\mathrm{Ag})\end{array}$ & $\begin{array}{l}\text { Silver, } \\
\text { dis- } \\
\text { solved } \\
(\mu \mathrm{g} / \mathrm{L} \text { as } \\
\mathrm{Ag})\end{array}$ & $\begin{array}{c}\text { Uranium, } \\
\text { dis- } \\
\text { solved } \\
(\mu \mathrm{g} / \mathrm{L} \text { as } \\
\mathbf{U})\end{array}$ & $\begin{array}{c}\text { Zinc, } \\
\text { total } \\
\text { recov- } \\
\text { erable } \\
(\mu \mathrm{g} / \mathrm{L} \text { as } \\
\mathrm{Zn})\end{array}$ & $\begin{array}{c}\text { Zinc, } \\
\text { dis- } \\
\text { solved } \\
(\mu \mathrm{g} / \mathrm{L} \text { as } \\
\mathrm{Zn})\end{array}$ \\
\hline
\end{tabular}

\begin{tabular}{|c|c|c|c|c|c|c|c|c|c|c|c|}
\hline \multirow[b]{2}{*}{33} & \multicolumn{11}{|c|}{ CATARACT CREEK-Continued } \\
\hline & $10-18-96$ & $<.1$ & $<.1$ & $<1$ & $<1$ & $<1$ & $<1$ & $<1$ & $<1$ & 20 & 19 \\
\hline & $05-20-97$ & $<.1$ & -- & $<1$ & $<1$ & $<1$ & $<1$ & $<1$ & $<1$ & 60 & 46 \\
\hline \multirow[t]{2}{*}{34} & $10-18-96$ & $<.1$ & $<.1$ & $<1$ & $<1$ & $<1$ & $<1$ & $<1$ & $<1$ & $<10$ & 10 \\
\hline & $05-20-97$ & $<.1$ & -- & $<1$ & $<1$ & $<\mathbf{l}$ & $<1$ & $<1$ & $<1$ & 30 & 29 \\
\hline \multirow[t]{2}{*}{35} & $10-16-96$ & -- & -- & -- & - & -- & -- & - & -- & $<10$ & -- \\
\hline & $05-20-97$ & -- & -- & -- & -- & -- & -- & -- & -. & 20 & -. \\
\hline \multirow[t]{2}{*}{36} & $10-16-96$ & -- & -- & -- & -- & -- & -. & -- & -- & $<10$ & - \\
\hline & $05-20-97$ & -- & - & -- & -- & -- & -- & -- & -. & 30 & - \\
\hline \multirow[t]{2}{*}{37} & $05-20-97$ & -- & -- & -- & -- & -- & -- & -- & - & 10 & - \\
\hline & $09-26-97$ & -- & - & -- & -- & -- & -- & -- & -- & $<10$ & - \\
\hline \multirow[t]{2}{*}{38} & $10-16-96$ & -. & -- & -- & - & -- & -- & -- & -- & 140 & -. \\
\hline & $05-20-97$ & -- & -- & -- & -- & -- & -- & -- & - & 50 & - \\
\hline \multirow[t]{2}{*}{39} & $10-16-96$ & -- & -- & -- & - & -- & -- & -- & -- & 100 & -- \\
\hline & $05-20-97$ & $-\cdot$ & -- & -- & -. & -- & -. & -- & - & 120 & - \\
\hline \multirow[t]{3}{*}{40} & $10-16-96$ & -- & -- & -- & -- & -- & -- & -- & -- & 210 & - \\
\hline & $05-21-97$ & -- & -- & $<1$ & -- & $<1$ & - & $<1$ & $<1$ & 260 & 258 \\
\hline & $09-24-97$ & -- & -. & $<1$ & - & $<1$ & -- & $<1$ & $<1$ & 200 & 202 \\
\hline \multirow[t]{12}{*}{41} & $10-16-96$ & -- & -- & -- & -- & - & -- & -- & -- & 70 & -- \\
\hline & $05-13-97$ & -- & -- & -- & -- & - & -- & -- & -- & 90 & -- \\
\hline & $05-21-97$ & -- & -- & $<1$ & -- & $<1$ & -- & $<1$ & $<1$ & 60 & 50 \\
\hline & $07-23-97$ & -- & -- & $<1$ & -- & $<1$ & -- & $<1$ & $<1$ & 40 & 34 \\
\hline & $09-24-97$ & -- & -- & $<1$ & -- & $<1$ & -- & $<1$ & $<1$ & 60 & 63 \\
\hline & $02-12-98$ & -- & -- & $<1$ & + & $<1$ & -- & $<1$ & $<1$ & 80 & 75 \\
\hline & $04-24-98$ & -- & -- & $<1$ & -- & $<1$ & -- & $<1$ & $<1$ & 80 & 55 \\
\hline & $05-06-98$ & -- & -. & $<1$ & -. & $<1$ & -- & $<1$ & $<1$ & 50 & 36 \\
\hline & $06-26-98$ & -- & -- & $<1$ & -- & $<1$ & -- & $<1$ & $<1$ & 50 & 45 \\
\hline & $09-22-98$ & -- & -- & $<1$ & -- & $<1$ & -- & $<1$ & $<1$ & 60 & 57 \\
\hline & $05-24-99$ & -- & -- & -- & -- & - & -- & -- & -- & 63 & 41 \\
\hline & $08-05-99$ & -- & -- & - & -- & -- & -- & -- & -- & $<40$ & 47 \\
\hline 42 & $09-28-98$ & -. & -- & $<1$ & -- & $<1$ & -- & $<1$ & $<1$ & -- & 41 \\
\hline \multirow[t]{15}{*}{43} & $10-16-96$ & $<.1$ & $<.1$ & $<1$ & 3 & 3 & $<1$ & $<1$ & 1 & 4,300 & 3,750 \\
\hline & $05-15-97$ & $<.1$ & -- & $<1$ & 2 & $<1$ & $<1$ & $<1$ & 1 & 1,100 & 928 \\
\hline & $09-24-97$ & -- & -- & $<1$ & -. & 4 & -- & $<1$ & 1 & 4,300 & 4,030 \\
\hline & $02-12-98$ & -. & -- & $<1$ & -- & 3 & -- & $<1$ & 1 & 3,000 & 2,790 \\
\hline & $04-24-98$ & -- & -- & $<1$ & -- & 2 & -- & $<1$ & $<1$ & 1,400 & 1,200 \\
\hline & $05-06-98$ & -- & -- & $<1$ & -- & 1 & -- & $<1$ & $<1$ & 1,000 & 885 \\
\hline & $06-26-98$ & -- & -- & $<1$ & -- & 1 & -- & $<1$ & $<1$ & 930 & 685 \\
\hline & $07-21-98$ & -- & -. & $<1$ & .. & 2 & -- & $<1$ & 1 & 2,100 & 1,750 \\
\hline & $07-22-98$ & -- & -- & - & -- & -- & -- & -- & - & - & 1,910 \\
\hline & $09-22-98$ & -- & -- & $<1$ & -- & 4 & -- & $<1$ & 1 & 4,300 & 4,310 \\
\hline & $05-24-99$ & -- & -- & - & -- & -- & -- & -- & -- & 1,500 & 902 \\
\hline & 08-02-99 & -- & -- & - & -- & -- & -- & - & -- & -- & 5,730 \\
\hline & 08-03-99 & -- & -- & -. & -- & -- & -- & -- & -- & -- & 4,960 \\
\hline & $08-04-99$ & -- & -- & -- & - & -- & -- & -- & -- & 4,800 & 4,940 \\
\hline & 08-05-99 & -- & -- & -. & - & -- & -- & -- & -- & -- & 3,020 \\
\hline
\end{tabular}


Table 2. Water-quality data for streams in the Boulder River watershed, Montana, 1996-99 (Continued)

\begin{tabular}{|c|c|c|c|c|c|c|c|c|c|c|}
\hline $\begin{array}{c}\text { Site } \\
\text { num- } \\
\text { ber } \\
\text { (fig. 2) }\end{array}$ & Station name & Date & Time & $\begin{array}{c}\text { Dis- } \\
\text { charge, } \\
\text { instanta- } \\
\text { neous } \\
\left(\mathrm{ft}^{3} / \mathrm{s}\right)\end{array}$ & $\begin{array}{c}\text { Sedi- } \\
\text { ment, } \\
\text { sus- } \\
\text { pended } \\
(\mathrm{mg} / \mathrm{L})\end{array}$ & $\begin{array}{c}\text { Sedi- } \\
\text { ment, } \\
\text { sus- } \\
\text { pended, } \\
\text { diameter } \\
\text { (percent } \\
\text { finer } \\
\text { than } \\
\mathbf{0 . 0 6 2} \\
\text { mm) }\end{array}$ & $\begin{array}{c}\text { Spe- } \\
\text { cific } \\
\text { con- } \\
\text { duct- } \\
\text { ance, } \\
\text { field } \\
(\mu \mathrm{S} / \mathrm{cm})\end{array}$ & $\begin{array}{c}\mathrm{pH}, \\
\text { field } \\
\text { (stan- } \\
\text { dard } \\
\text { units) }\end{array}$ & $\begin{array}{c}\text { Tem- } \\
\text { pera- } \\
\text { ture, } \\
\text { water } \\
\left({ }^{\circ} \mathrm{C}\right)\end{array}$ & $\begin{array}{c}\text { Hard- } \\
\text { ness } \\
(\mathrm{mg} / \mathrm{L} \\
\text { as } \\
\left.\mathrm{CaCO}_{3}\right)\end{array}$ \\
\hline
\end{tabular}

45 Deer Creek near Basin

$46 \quad$ Big Limber Gulch near Basin

47 Cataract Creek at Basin

49

High Ore Creek above Comet Mine, near Basin
Cataract Creek below Uncle Sam Gulch, near Basin

\section{CATARACT CREEK-Continued}

\begin{tabular}{|c|c|c|c|c|c|c|c|c|}
\hline $10-16-96$ & 1510 & 3.8 & 4 & 88 & 130 & 7.6 & 1.0 & 55 \\
\hline $05-15-97$ & 1330 & 186 & 48 & 50 & 52 & 7.5 & 5.0 & 19 \\
\hline $07-23-97$ & 1445 & 17 & 4 & 35 & 92 & 7.9 & 16.5 & 37 \\
\hline $09-24-97$ & 1220 & 4.5 & 5 & 24 & 129 & 8.0 & 8.0 & 57 \\
\hline $02-12-98$ & 0940 & 3.6 & 3 & 38 & 127 & 7.6 & 0.0 & 53 \\
\hline $04-24-98$ & 1230 & 35 & 19 & 70 & 80 & 7.8 & 2.0 & 34 \\
\hline $05-06-98$ & 1415 & 82 & 17 & 38 & 49 & 7.2 & 7.0 & 20 \\
\hline $06-26-98$ & 1145 & 122 & 18 & 37 & 47 & 7.8 & 6.5 & 21 \\
\hline $07-21-98$ & 1310 & 20 & 10 & 32 & 87 & 7.9 & 14.0 & 36 \\
\hline $07-22-98$ & 1100 & -- & -- & -- & 89 & 7.8 & 11.0 & -- \\
\hline $07-23-98$ & 1000 & -- & - & -- & 89 & 8.2 & 11.0 & -- \\
\hline $07-24-98$ & 1015 & -- & -- & -- & 91 & 8.1 & 11.0 & -- \\
\hline $09-22-98$ & 1400 & 3.6 & -- & -- & 129 & 7.8 & -- & 55 \\
\hline $05-24-99$ & 1700 & 129 & 52 & 65 & 47 & 7.5 & 7.5 & 18 \\
\hline $08-02-99$ & 1610 & -- & -- & -- & 125 & 7.9 & 18.0 & -- \\
\hline 08-03-99 & 1350 & -- & -- & -- & 112 & 8.0 & 15.5 & -- \\
\hline 08-04-99 & 1330 & 4.6 & 1 & 82 & 118 & 7.9 & 14.5 & 48 \\
\hline $08-05-99$ & 1305 & -- & -- & -- & 106 & 7.8 & 14.0 & -- \\
\hline $10-16-96$ & 1712 & 1.0 & - & - & 217 & -- & 2.0 & - \\
\hline $05-13-97$ & 1645 & 8.5 & -- & -- & 62 & -- & 4.0 & -- \\
\hline $10-16-96$ & 1355 & -- & -- & -- & 313 & -- & 1.5 & - \\
\hline $05-13-97$ & 1730 & .73 & -- & -- & 219 & -- & 12.0 & -- \\
\hline $10-15-96$ & 1645 & 3.5 & 3 & 59 & 158 & 7.9 & 4.5 & 69 \\
\hline $12-04-96$ & 1020 & 5.4 & 1 & 75 & 140 & 7.5 & 0.0 & 59 \\
\hline $02-11-97$ & 1330 & 3.8 & -- & -- & 147 & 7.6 & 0.0 & 62 \\
\hline $04-18-97$ & 1325 & 13 & 10 & 70 & 128 & 7.9 & 5.5 & 54 \\
\hline $05-08-97$ & 1200 & 46 & 15 & 70 & 91 & 7.7 & 3.0 & 36 \\
\hline $05-15-97$ & 0930 & 158 & 51 & 38 & 50 & 7.1 & 4.0 & 19 \\
\hline $05-22-97$ & 1000 & 134 & 18 & 45 & 53 & 6.8 & 5.5 & 18 \\
\hline $06-03-97$ & 1200 & 123 & 13 & 51 & 49 & 7.4 & 8.0 & 19 \\
\hline $06-11-97$ & 1240 & 168 & 48 & 37 & 46 & 7.2 & 10.0 & 19 \\
\hline $06-17-97$ & 1230 & 73 & 5 & 66 & 65 & 7.7 & 12.5 & 26 \\
\hline $07-18-97$ & 1045 & 27 & 2 & 79 & 94 & 7.9 & 11.0 & 36 \\
\hline $09-24-97$ & 1410 & 4.6 & 3 & 31 & 146 & 8.0 & 11.5 & 69 \\
\hline $02-09-98$ & 1310 & 3.8 & 1 & 75 & 148 & 7.5 & 0.0 & 63 \\
\hline $04-24-98$ & 1415 & 32 & 16 & 86 & 90 & 8.0 & 4.0 & 38 \\
\hline $05-05-98$ & 1200 & 77 & 14 & 69 & 54 & 7.7 & 3.5 & 22 \\
\hline $09-22-98$ & 1530 & 3.7 & 4 & 74 & 143 & 7.8 & 10.0 & 66 \\
\hline $05-25-99$ & 1250 & 97 & 14 & 51 & 48 & 7.5 & 7.0 & 18 \\
\hline $09-14-99$ & 1140 & 2.6 & 2 & 31 & 153 & 7.8 & 10.0 & 67 \\
\hline \multicolumn{9}{|c|}{ HIGH ORE CREEK } \\
\hline $10-17-96$ & 1400 & $\cdots$ & 9 & 75 & 149 & 7.6 & 0.0 & 67 \\
\hline $05-09-97$ & 0925 & 1.5 & 3 & 90 & 105 & 7.9 & 4.0 & 44 \\
\hline $09-23-98$ & 1105 & .27 & 4 & 59 & 123 & 8.2 & 5.5 & 59 \\
\hline $09-15-99$ & 1000 & .13 & 8 & 92 & 129 & 8.1 & 6.0 & 58 \\
\hline $10-17-96$ & 1455 & - & - & -- & 146 & -- & 1.0 & -- \\
\hline $05-08-97$ & 1350 & 2.1 & - & -- & 113 & -- & 8.5 & -- \\
\hline
\end{tabular}


Table 2. Water-quality data for streams in the Boulder River watershed, Montana, 1996-99 (Continued)

\begin{tabular}{|c|c|c|c|c|c|c|c|c|c|c|}
\hline $\begin{array}{c}\text { Site } \\
\text { num- } \\
\text { ber } \\
\text { (fig. 2) }\end{array}$ & Date & $\begin{array}{c}\text { Cal- } \\
\text { cium, } \\
\text { dissolved } \\
\text { (mg/L } \\
\text { as Ca) }\end{array}$ & $\begin{array}{c}\text { Magne- } \\
\text { sium, } \\
\text { dissolved } \\
\text { (mg/L } \\
\text { as } \mathbf{M g} \text { ) }\end{array}$ & $\begin{array}{c}\text { Sodium, } \\
\text { dissolved } \\
(\mathrm{mg} / \mathrm{L} \\
\text { as } \mathrm{Na})\end{array}$ & $\begin{array}{l}\text { Potas- } \\
\text { sium, } \\
\text { dissolved } \\
\text { (mg/L } \\
\text { as K) }\end{array}$ & $\begin{array}{c}\text { ANC, } \\
\text { lab } \\
(\mathrm{mg} / \mathrm{L} \\
\left.\text { as } \mathrm{CaCO}_{3}\right)\end{array}$ & $\begin{array}{c}\text { Sulfate, } \\
\text { dissolved } \\
(\mathrm{mg} / \mathrm{L} \\
\left.\text { as } \mathrm{SO}_{4}\right)\end{array}$ & $\begin{array}{c}\text { Chloride, } \\
\text { dissolved } \\
(\mathrm{mg} / \mathrm{L} \\
\text { as } \mathrm{Cl})\end{array}$ & $\begin{array}{c}\text { Fluoride, } \\
\text { dissolved } \\
(\mathrm{mg} / \mathbf{L} \\
\text { as F) }\end{array}$ & $\begin{array}{c}\text { Silica, } \\
\text { dissolved } \\
(\mathrm{mg} / \mathrm{L} \\
\left.\text { as } \mathrm{SiO}_{2}\right)\end{array}$ \\
\hline
\end{tabular}

\begin{tabular}{|c|c|c|c|c|c|c|c|c|c|c|c|}
\hline \multicolumn{12}{|c|}{ CATARACT CREEK--Continued } \\
\hline \multirow[t]{18}{*}{44} & $10-16-96$ & 17 & 3.1 & 3.4 & 1.0 & 44 & 21 & .70 & $<.10$ & 15 & 88 \\
\hline & $05-15-97$ & 5.6 & 1.1 & 1.6 & .83 & 12 & 9.5 & .44 & $<.10$ & 10 & 37 \\
\hline & $07-23-97$ & 11 & 2.1 & 2.5 & .88 & 1.8 & 11 & .37 & $<.10$ & 14 & 44 \\
\hline & $09-24-97$ & 17 & 3.3 & 3.4 & 1.0 & 43 & 19 & .59 & $<.10$ & 16 & 88 \\
\hline & $02-12-98$ & 16 & 3.1 & 3.3 & 1.0 & 43 & 18 & .64 & $<.10$ & 15 & 84 \\
\hline & $04-24-98$ & 10 & 2.0 & 2.1 & 1.7 & 30 & 9.0 & .56 & $<.10$ & 11 & 55 \\
\hline & $05-06-98$ & 6.2 & 1.1 & 1.6 & .83 & 17 & 5.6 & .34 & $<.10$ & 9.5 & 36 \\
\hline & $06-26-98$ & 6.5 & 1.2 & 1.9 & .54 & 19 & 5.9 & .19 & $<.10$ & 11 & 39 \\
\hline & $07-21-98$ & 11 & 2.0 & 2.6 & .88 & 32 & 9.0 & .35 & $<.10$ & 17 & 62 \\
\hline & $07-22-98$ & -- & -- & -- & -- & -- & -- & -- & -- & - & -- \\
\hline & $07-23-98$ & -- & -- & -- & -- & -- & -- & -- & -- & -- & -- \\
\hline & $07-24-98$ & -- & -- & -- & -- & -- & -- & -- & -- & - & -- \\
\hline & $09-22-98$ & 17 & 3.2 & 3.3 & .81 & 44 & 20 & .46 & .10 & 16 & 88 \\
\hline & $05-24-99$ & 5.4 & 1.1 & 1.6 & .73 & 14 & 6.9 & .23 & -- & - & \\
\hline & $08-02-99$ & - & - & -- & -- & -- & -- & -- & -- & -- & -- \\
\hline & $08-03-99$ & -- & -. & -- & -- & - & -- & -- & -- & -- & -- \\
\hline & $08-04-99$ & 15 & 2.8 & 3.2 & 1.1 & 39 & 18 & .41 & -- & - & - \\
\hline & $08-05-99$ & -- & -- & - & -- & -- & -- & -- & -- & -- & -- \\
\hline \multirow[t]{2}{*}{45} & $10-16-96$ & -- & -- & -- & -- & -- & -- & -- & - & -- & - \\
\hline & $05-13-97$ & -- & -- & -- & -- & -- & -- & -- & -- & -- & -- \\
\hline \multirow[t]{2}{*}{46} & $10-16-96$ & -- & -- & - & -- & - & - & -- & -- & -- & -- \\
\hline & $05-13-97$ & -- & -- & - & -- & -- & -- & - & - & - & -- \\
\hline \multirow[t]{19}{*}{47} & $10-15-96$ & 21 & 4.0 & 3.7 & 1.2 & 51 & 24 & 1.8 & $<.10$ & 15 & 102 \\
\hline & $12-04-96$ & 18 & 3.5 & 3.7 & 1.0 & 49 & 22 & .60 & $<.10$ & 15 & 94 \\
\hline & $02-11-97$ & 19 & 3.5 & -- & -- & - & -- & -- & - & -- & - \\
\hline & $04-18-97$ & 17 & 3.1 & 3.2 & 1.2 & 43 & 19 & .60 & $<.10$ & 15 & 84 \\
\hline & $05-08-97$ & 11 & 2.1 & 2.6 & .95 & 29 & 13 & .80 & $<.10$ & 13 & 60 \\
\hline & $05-15-97$ & 5.7 & 1.1 & 1.6 & .75 & 15 & 7.1 & .49 & $<.10$ & 9.7 & 36 \\
\hline & $05-22-97$ & 5.6 & 1.1 & 1.6 & .66 & 11 & 6.2 & 3.0 & $<.10$ & 11 & 36 \\
\hline & $06-03-97$ & 5.7 & 1.1 & 1.7 & .61 & 16 & 6.2 & .28 & $<.10$ & 11 & 36 \\
\hline & $06-11-97$ & 5.9 & 1.0 & 1.6 & .62 & 16 & 4.5 & .21 & $<.10$ & 9.8 & 34 \\
\hline & $06-17-97$ & 7.8 & 1.5 & 2.1 & .71 & 22 & 6.7 & .58 & $<.10$ & 12 & 45 \\
\hline & $07-18-97$ & 11 & 2.1 & 2.4 & .88 & 35 & 9.8 & .38 & $<.10$ & 14 & 62 \\
\hline & $09-24-97$ & 21 & 4.0 & 3.7 & 1.1 & 51 & 21 & .67 & $<.10$ & 16 & 98 \\
\hline & $02-09-98$ & 19 & 3.8 & 3.6 & 1.0 & -- & 20 & 4.6 & $<.10$ & 15 & -- \\
\hline & $04-24-98$ & 11 & 2.2 & 2.3 & 1.6 & 33 & 10 & .61 & $<.10$ & 11 & 60 \\
\hline & $05-05-98$ & 6.8 & 1.3 & 1.6 & .94 & 19 & 6.1 & .31 & $<.10$ & 9.4 & 38 \\
\hline & $09-22-98$ & 20 & 3.8 & 3.6 & 1.1 & 52 & 21 & .58 & $<.10$ & 16 & 98 \\
\hline & $05-25-99$ & 5.5 & 1.1 & 1.6 & .69 & 16 & 6.1 & .28 & - & - & - \\
\hline & $09-14-99$ & 20 & 3.9 & 3.7 & 1.2 & 52 & 22 & .83 & -- & - & - \\
\hline & \multicolumn{11}{|c|}{ HIGH ORE CREEK } \\
\hline \multirow[t]{4}{*}{49} & $10-17-96$ & 21 & 3.5 & 4.5 & .90 & 66 & 9.6 & .90 & .10 & 18 & 98 \\
\hline & $05-09-97$ & 14 & 2.2 & 3.2 & .75 & 45 & 7.7 & 1.1 & $<.10$ & 14 & 70 \\
\hline & $09-23-98$ & 18 & 3.0 & 3.9 & .69 & 58 & 7.9 & .51 & .10 & 18 & 87 \\
\hline & $09-15-99$ & 18 & 3.0 & 4.0 & .94 & 59 & 7.9 & .73 & - & -- & -- \\
\hline \multirow[t]{2}{*}{50} & $10-17-96$ & - & -- & -- & -- & -- & -- & -- & -- & -- & -- \\
\hline & $05-08-97$ & - & - & - & -- & -- & - & -- & -- & -- & -- \\
\hline
\end{tabular}


Table 2. Water-quality data for streams in the Boulder River watershed, Montana, 1996-99 (Continued)

\begin{tabular}{|c|c|c|c|c|c|c|c|}
\hline $\begin{array}{c}\text { Site } \\
\text { num- } \\
\text { ber } \\
\text { (fig. 2) }\end{array}$ & Date & $\begin{array}{c}\text { Nitrite, } \\
\text { dissolved } \\
(\mathbf{m g} / \mathbf{L} \\
\text { as } \mathbf{N})\end{array}$ & $\begin{array}{c}\text { Nitrite } \\
\text { plus } \\
\text { nitrate, } \\
\text { dissolved } \\
\text { (mg/L } \\
\text { as } \mathbf{N})\end{array}$ & $\begin{array}{c}\text { Ammonia, } \\
\text { dissolved } \\
(\mathrm{mg} / \mathbf{L} \\
\text { as } \mathbf{N})\end{array}$ & $\begin{array}{c}\text { Ammonia plus } \\
\text { organic } \\
\text { nitrogen, } \\
\text { total } \\
\text { (mg/L } \\
\text { as } \mathrm{N})\end{array}$ & $\begin{array}{c}\text { Phos- } \\
\text { phorus, } \\
\text { total } \\
\text { (mg/L } \\
\text { as P) }\end{array}$ & $\begin{array}{l}\text { Phos- } \\
\text { phorus, } \\
\text { ortho- } \\
\text { phosphate, } \\
\text { dissolved } \\
\text { (mg/L } \\
\text { as P) }\end{array}$ \\
\hline \multicolumn{8}{|c|}{ CATARACT CREEK-Continued } \\
\hline \multirow[t]{18}{*}{44} & $10-16-96$ & -- & -- & -- & -- & -- & -- \\
\hline & $05-15-97$ & -- & -- & -- & -- & -- & -- \\
\hline & $07-23-97$ & $<.01$ & $<.05$ & $<.015$ & -- & -- & $<.010$ \\
\hline & $09-24-97$ & $<.01$ & $<.05$ & $<.015$ & $<.2$ & $<.010$ & $<.010$ \\
\hline & $02-12-98$ & $<.01$ & .10 & .029 & -- & -- & .015 \\
\hline & $04-24-98$ & -- & -- & -- & -- & -. & -- \\
\hline & $05-06-98$ & -- & -- & -- & -- & - & -- \\
\hline & $06-26-98$ & -- & -- & -- & -- & -- & - \\
\hline & $07-21-98$ & .01 & $<.05$ & .045 & -- & -- & $<.010$ \\
\hline & $07-22-98$ & -- & -- & -- & -- & -- & -- \\
\hline & $07-23-98$ & -- & - & -- & -- & -- & -- \\
\hline & $07-24-98$ & -- & -- & -- & -- & -- & -- \\
\hline & $09-22-98$ & -- & -- & -- & -- & -- & -- \\
\hline & $05-24-99$ & -- & - & -- & -. & -- & -- \\
\hline & $08-02-99$ & -- & -- & -. & -- & -- & -- \\
\hline & 08-03-99 & -- & -- & -- & -- & -- & -- \\
\hline & 08-04-99 & - & - & -- & -- & -- & - \\
\hline & $08-05-99$ & - & - & -- & -- & -- & - \\
\hline \multirow[t]{2}{*}{45} & $10-16-96$ & -- & -- & -- & -- & -. & -. \\
\hline & $05-13-97$ & -- & -- & -- & -. & -- & - \\
\hline \multirow[t]{2}{*}{46} & $10-16-96$ & -- & - & -- & -- & -- & - \\
\hline & $05-13-97$ & - & - & -- & -- & -- & - \\
\hline \multirow[t]{19}{*}{47} & $10-15-96$ & -- & -- & -- & - & -- & -- \\
\hline & $12-04-96$ & .01 & .06 & .040 & -- & -. & $<.010$ \\
\hline & $02-11-97$ & -- & -- & -- & -- & -- & -- \\
\hline & $04-18-97$ & - & - & -- & -- & -- & -- \\
\hline & $05-08-97$ & -- & -- & -- & -- & -- & -- \\
\hline & $05-15-97$ & $<.01$ & $<.05$ & $<.015$ & -- & -- & $<.010$ \\
\hline & $05-22-97$ & $<.01$ & $<.05$ & $<.015$ & -- & -- & $<.010$ \\
\hline & $06-03-97$ & -- & -- & -- & -- & -- & - \\
\hline & $06-11-97$ & - & -- & -- & -- & -- & -- \\
\hline & $06-17-97$ & -- & -- & -- & -- & -- & -- \\
\hline & $07-18-97$ & $<.01$ & $<.05$ & $<.015$ & -- & -- & $<.010$ \\
\hline & $09-24-97$ & $<.01$ & $<.05$ & $<.015$ & $<.2$ & $<.010$ & $<.010$ \\
\hline & $02-09-98$ & $<.01$ & .09 & .050 & - & -- & .014 \\
\hline & $04-24-98$ & - & - & - & - & -- & -- \\
\hline & $05-05-98$ & -- & -- & - & -- & -- & -- \\
\hline & $09-22-98$ & -- & -- & -- & .15 & $<.05$ & - \\
\hline & $05-25-99$ & -- & -- & -- & -- & -- & -- \\
\hline & 09-14-99 & -- & -- & -- & - & -- & -- \\
\hline & \multicolumn{7}{|c|}{ HIGH ORE CREEK } \\
\hline \multirow[t]{4}{*}{49} & $10-17-96$ & -. & -- & -- & -- & -- & -- \\
\hline & $05-09-97$ & - & -- & -- & -- & - & -- \\
\hline & $09-23-98$ & - & - & -- & .10 & .084 & -- \\
\hline & $09-15-99$ & -- & -- & -- & - & -- & -- \\
\hline \multirow[t]{2}{*}{50} & $10-17-96$ & -- &.- & - & -- & -- & - \\
\hline & $05-08-97$ & -- & -- & -- & -- & -- & -- \\
\hline
\end{tabular}


Table 2. Water-quality data for streams in the Boulder River watershed, Montana, 1996-99 (Continued)

\begin{tabular}{|c|c|c|c|c|c|c|c|c|c|c|c|c|}
\hline $\begin{array}{c}\text { Site } \\
\text { num- } \\
\text { ber } \\
\text { (fig. 2) }\end{array}$ & Date & $\begin{array}{l}\text { Alumi- } \\
\text { num, } \\
\text { total } \\
\text { recov- } \\
\text { erable } \\
(\mu \mathrm{g} / \mathrm{L} \text { as } \\
\text { Al) }\end{array}$ & $\begin{array}{l}\text { Alumi- } \\
\text { num, } \\
\text { dis- } \\
\text { solved } \\
(\mu \mathrm{g} / \mathbf{L} \text { as } \\
\text { Al) }\end{array}$ & $\begin{array}{l}\text { Anti- } \\
\text { mony, } \\
\text { dis- } \\
\text { solved } \\
(\mu \mathrm{g} / \mathrm{L} \text { as } \\
\text { Sb) }\end{array}$ & $\begin{array}{c}\text { Arsenic, } \\
\text { total } \\
\text { recov- } \\
\text { erable } \\
(\mu g / L \text { as } \\
\text { As) }\end{array}$ & $\begin{array}{c}\text { Arsenic, } \\
\text { dis- } \\
\text { solved } \\
(\mu \mathrm{g} / \mathrm{L} \text { as } \\
\text { As) }\end{array}$ & $\begin{array}{c}\text { Barium, } \\
\text { dis- } \\
\text { solved } \\
(\mu \mathrm{g} / \mathbf{L} \text { as } \\
\text { Ba })\end{array}$ & $\begin{array}{l}\text { Beryl- } \\
\text { lium, } \\
\text { dis- } \\
\text { solved } \\
(\mu \mathrm{g} / \mathrm{L} \text { as } \\
\text { Be })\end{array}$ & $\begin{array}{l}\text { Cad- } \\
\text { mium, } \\
\text { total } \\
\text { recov- } \\
\text { erable } \\
(\mu \mathrm{g} / \mathrm{L} \text { as } \\
\text { Cd })\end{array}$ & $\begin{array}{c}\text { Cad- } \\
\text { mium, } \\
\text { dis- } \\
\text { solved } \\
(\mu \mathrm{g} / \mathrm{L} \text { as } \\
\text { Cd) }\end{array}$ & $\begin{array}{l}\text { Chro- } \\
\text { mium, } \\
\text { total } \\
\text { recov- } \\
\text { erable } \\
(\mu \mathrm{g} / \mathrm{L} \text { as } \\
\text { Cr })\end{array}$ & $\begin{array}{c}\text { Chro- } \\
\text { mium, } \\
\text { dis- } \\
\text { solved } \\
(\mu \mathrm{g} / \mathrm{L} \text { as } \\
\text { Cr) }\end{array}$ \\
\hline \multicolumn{13}{|c|}{ CATARACT CREEK-Continued } \\
\hline \multirow[t]{18}{*}{44} & $10-16-96$ & -- & 9.5 & $<1$ & 3 & 2 & 17 & $<1$ & 8 & 8.0 & $<1$ & $<1$ \\
\hline & $05-15-97$ & 910 & 81 & $<1$ & 93 & 4 & 11 & $<1$ & 5 & 3.4 & $<1$ & $<1$ \\
\hline & $07-23-97$ & 100 & 29 & $<1$ & 10 & 3 & 16 & $<1$ & 4 & 3.8 & -- & 1 \\
\hline & $09-24-97$ & 140 & 9.2 & $<1$ & 5 & 2 & 18 & $<1$ & 9 & 8.9 & - & $<1$ \\
\hline & $02-12-98$ & 30 & 5.8 & $<1$ & 3 & 2 & 16 & $<1$ & 5 & 5.6 & -- & $<1$ \\
\hline & $04-24-98$ & 330 & 32 & $<1$ & 20 & 2 & 14 & $<1$ & 3 & 2.3 & -- & $<1$ \\
\hline & $05-06-98$ & 330 & 57 & $<1$ & 12 & 3 & 11 & $<1$ & 1 & 1.1 & -- & $<1$ \\
\hline & $06-26-98$ & 270 & 50 & $<1$ & 15 & 2 & 10 & $<1$ & 2 & 1.2 & - & $<1$ \\
\hline & $07-21-98$ & 110 & 25 & $<1$ & 11 & 3 & 16 & $<1$ & 3 & 3.3 & -- & $<1$ \\
\hline & $07-22-98$ & -- & -- & - & -- & -- & -- & -- & -- & 4.6 & -- & $<1$ \\
\hline & $07-23-98$ & - & -- & -- & -- & -- & -- & - & - & 4.2 & -. & $<1$ \\
\hline & $07-24-98$ & -- & -. & -- & -- & -- & - & - & -- & 4.7 & -- & $<1$ \\
\hline & $09-22-98$ & 120 & 7.0 & $<1$ & 4 & 2 & 18 & $<1$ & 10 & 9.9 & -. & $<1$ \\
\hline & $05-24-99$ & 1,110 & 56 & -- & 180 & 4 & -- & -- & 5 & 2.0 & .. & -- \\
\hline & $08-02-99$ & -- & -- & -- & -- & -- & -- & -- & -- & 9.6 & - & -- \\
\hline & 08-03-99 & -- & -- & -- & -- & -- & -- & -- & - & 7.4 & -- & -- \\
\hline & 08-04-99 & 70 & 9.5 & - & 4 & 3 & -- & -- & 10 & 9.3 & $\ldots$ & - \\
\hline & 08-05-99 & -- & -- & -- & -- & -- & -- & -- & -- & 6.7 & - & -- \\
\hline \multirow[t]{2}{*}{45} & $10-16-96$ & - & -- & - & -- & -- & -- & - & - & -- & - & - \\
\hline & $05-13-97$ & -- & $-\cdot$ & -- & -- & - & -- & -- & -- & -- & -- & -- \\
\hline \multirow[t]{2}{*}{46} & $10-16-96$ & -- & -- & -- & -- & -- & -- & -- & -- & -. & - & -- \\
\hline & $05-13-97$ & -- & -- & - & -- & -- & -- & -- & -- & - & -- & -- \\
\hline \multirow[t]{18}{*}{47} & $10-15-96$ & -- & 13 & $<1$ & 5 & 4 & 21 & $<1$ & 5 & 4.9 & $<1$ & $<1$ \\
\hline & $12-04-96$ & -- & 6.5 & $<1$ & 5 & 3 & 18 & $<1$ & 3 & 3.3 & $<1$ & $<1$ \\
\hline & $02-11-97$ & -- & 6.5 & $<1$ & 3 & 3 & 20 & $<1$ & 3 & 3.0 & -- & $<1$ \\
\hline & $04-18-97$ & -- & 11 & $<1$ & 6 & 3 & 17 & $<1$ & 3 & 2.5 & -- & $<1$ \\
\hline & $05-08-97$ & 400 & 108 & $<1$ & 10 & 3 & 14 & $<1$ & 2 & 1.7 & -- & $<1$ \\
\hline & $05-15-97$ & 950 & 58 & $<1$ & 38 & 3 & 10 & $<1$ & 2 & 1.1 & $<1$ & $<1$ \\
\hline & $05-22-97$ & 330 & 85 & $<1$ & 19 & 4 & 10 & $<1$ & 1 & 1.3 & $<1$ & $<1$ \\
\hline & $06-03-97$ & 310 & 79 & $<1$ & 21 & 4 & 10 & $<1$ & 1 & 1.2 & -- & $<1$ \\
\hline & $06-11-97$ & 790 & 89 & $<1$ & 35 & 4 & 10 & $<1$ & 2 & 1.0 & -- & $<1$ \\
\hline & $06-17-97$ & 150 & 52 & $<1$ & 13 & 4 & 12 & $<1$ & 2 & 1.4 & -- & $<1$ \\
\hline & $07-18-97$ & -- & 29 & $<1$ & 8 & 4 & 16 & $<1$ & 2 & 2.4 & -- & $<1$ \\
\hline & $09-24-97$ & 40 & 11 & $<1$ & 5 & 3 & 21 & $<1$ & 5 & 5.0 & -- & $<1$ \\
\hline & $02-09-98$ & 10 & 5.8 & $<1$ & 3 & 3 & 18 & $<1$ & 3 & 2.9 & - & $<1$ \\
\hline & $04-24-98$ & 310 & 24 & $<1$ & 15 & 3 & 15 & $<1$ & 3 & 2.3 & -- & $<1$ \\
\hline & $05-05-98$ & 370 & 47 & $<1$ & 17 & 3 & 11 & $<1$ & 1 & 1.3 & -- & $<1$ \\
\hline & $09-22-98$ & 50 & 10 & $<1$ & 3 & 3 & 21 & $<1$ & 6 & 5.6 & - & $<1$ \\
\hline & $05-25-99$ & 410 & 66 & - & 21 & 3 & -- & -- & 2 & 1.2 & - & - \\
\hline & $09-14-99$ & 32 & 11 & -- & 5 & 4 & -- & -- & 6 & 5.8 & - & - \\
\hline \multicolumn{13}{|c|}{ HIGH ORE CREEK } \\
\hline \multirow[t]{4}{*}{49} & $10-17-96$ & -- & 3.5 & $<1$ & 2 & 2 & 21 & $<1$ & $<1$ & $<.1$ & $<1$ & $<1$ \\
\hline & $05-09-97$ & -- & 55 & $<1$ & 2 & 1 & 19 & $<1$ & $<1$ & $<.1$ & $<1$ & $<1$ \\
\hline & $09-23-98$ & 40 & 1.9 & $<1$ & 1 & 1 & 19 & $<1$ & $<1$ & $<.3$ & -- & $<1$ \\
\hline & $09-15-99$ & 170 & 4.9 & - & 2 & 2 & -- & -- & $<1$ & $<.3$ & -- & -- \\
\hline \multirow[t]{2}{*}{50} & $10-17-96$ & -- & -- & - & -- & -- & - & -- & -- & -- & - & -- \\
\hline & $05-08-97$ & -- & -- & -- & -- & -- & -- & -- & -- & - & - & -- \\
\hline
\end{tabular}


Table 2. Water-quality data for streams in the Boulder River watershed, Montana, 1996-99 (Continued)

\begin{tabular}{|c|c|c|c|c|c|c|c|c|c|c|c|}
\hline $\begin{array}{c}\text { Site } \\
\text { num- } \\
\text { ber } \\
\text { (fig. 2) }\end{array}$ & Date & $\begin{array}{c}\text { Cobalt, } \\
\text { dis- } \\
\text { solved } \\
(\mu \mathrm{g} / \mathrm{L} \text { as } \\
\text { Co })\end{array}$ & $\begin{array}{l}\text { Copper, } \\
\text { total recov- } \\
\text { erable } \\
(\mu \mathrm{g} / \mathrm{L} \text { as } \\
\mathrm{Cu})\end{array}$ & $\begin{array}{c}\text { Copper, } \\
\text { dis- } \\
\text { solved } \\
(\mu \mathrm{g} / \mathrm{L} \text { as } \\
\mathrm{Cu})\end{array}$ & $\begin{array}{c}\text { Iron, } \\
\text { total } \\
\text { recov- } \\
\text { erable } \\
(\mu \mathrm{g} / \mathrm{L} \text { as } \\
\text { Fe })\end{array}$ & $\begin{array}{c}\text { Iron, } \\
\text { dis- } \\
\text { solved } \\
(\mu \mathrm{g} / \mathrm{L} \text { as } \\
\text { Fe })\end{array}$ & $\begin{array}{c}\text { Lead, } \\
\text { total } \\
\text { recov- } \\
\text { erable } \\
(\mu \mathrm{g} / \mathrm{L} \text { as } \\
\text { Pb) }\end{array}$ & $\begin{array}{c}\text { Lead, } \\
\text { dis- } \\
\text { solved } \\
(\mu \mathrm{g} / \mathrm{L} \text { as } \\
\text { Pb) }\end{array}$ & $\begin{array}{l}\text { Lithium, } \\
\text { dis- } \\
\text { solved } \\
(\mu \mathrm{g} / \mathrm{L} \text { as } \\
\mathrm{Li})\end{array}$ & $\begin{array}{l}\text { Manga- } \\
\text { nese, } \\
\text { total } \\
\text { recov- } \\
\text { erable } \\
(\mu \mathrm{g} / \mathrm{L} \text { as } \\
\mathrm{Mn})\end{array}$ & $\begin{array}{c}\text { Manga- } \\
\text { nese, } \\
\text { dis- } \\
\text { solved } \\
(\mu \mathrm{g} / \mathrm{L} \text { as } \\
\text { Mn) }\end{array}$ \\
\hline
\end{tabular}

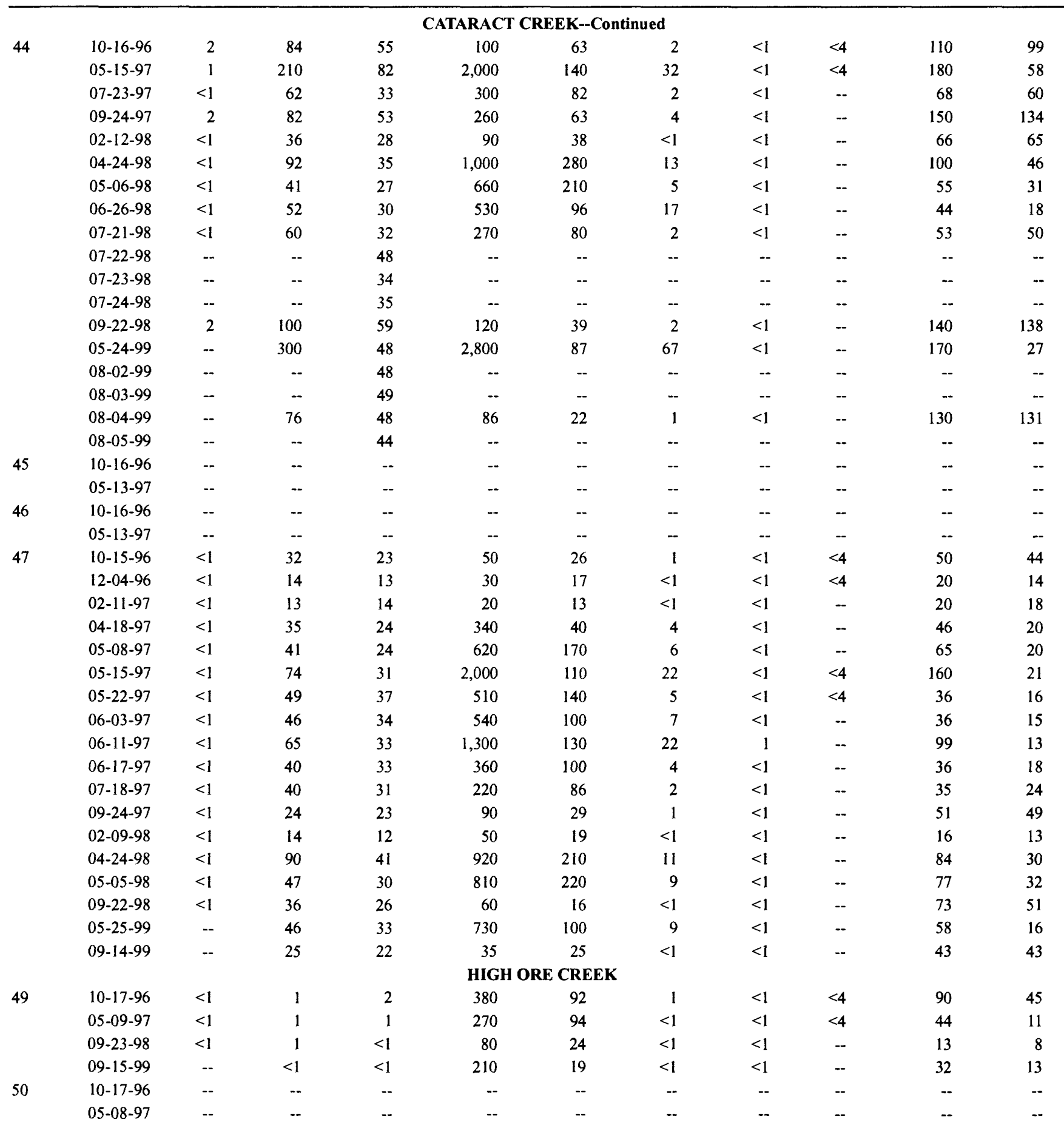


Table 2. Water-quality data for streams in the Boulder River watershed, Montana, 1996-99 (Continued)

\begin{tabular}{|c|c|c|c|c|c|c|c|c|c|c|c|}
\hline $\begin{array}{c}\text { Site } \\
\text { num- } \\
\text { ber } \\
\text { (fig. 2) }\end{array}$ & Date & $\begin{array}{c}\text { Mercury, } \\
\text { total } \\
\text { recov- } \\
\text { erable } \\
(\mu \mathrm{g} / \mathrm{L} \text { as } \\
\mathrm{Hg})\end{array}$ & $\begin{array}{c}\text { Mercury, } \\
\text { dis- } \\
\text { solved } \\
(\mu \mathrm{g} / \mathrm{L} \text { as } \\
\mathrm{Hg})\end{array}$ & $\begin{array}{c}\text { Molyb- } \\
\text { denum, } \\
\text { dis- } \\
\text { solved } \\
(\mu \mathrm{g} / \mathrm{L} \text { as } \\
\text { Mo) }\end{array}$ & $\begin{array}{c}\text { Nickel, } \\
\text { total } \\
\text { recov- } \\
\text { erable } \\
(\mu \mathrm{g} / \mathrm{L} \text { as } \\
\mathrm{Ni})\end{array}$ & $\begin{array}{c}\text { Nickel, } \\
\text { dis- } \\
\text { solved } \\
(\mu \mathrm{g} / \mathrm{L} \text { as } \\
\mathrm{Ni})\end{array}$ & $\begin{array}{l}\text { Silver, } \\
\text { total } \\
\text { recov- } \\
\text { erable } \\
(\mu \mathrm{g} / \mathrm{L} \text { as } \\
\mathrm{Ag})\end{array}$ & $\begin{array}{l}\text { Silver, } \\
\text { dis- } \\
\text { solved } \\
(\mu \mathrm{g} / \mathrm{L} \text { as } \\
\mathrm{Ag})\end{array}$ & $\begin{array}{c}\text { Uranium, } \\
\text { dis- } \\
\text { solved } \\
(\mu \mathrm{g} / \mathrm{L} \text { as } \\
\mathrm{U})\end{array}$ & $\begin{array}{c}\text { Zinc, } \\
\text { total } \\
\text { recov- } \\
\text { erable } \\
(\mu \mathrm{g} / \mathrm{L} \text { as } \\
\mathrm{Zn})\end{array}$ & $\begin{array}{c}\text { Zinc, } \\
\text { dis- } \\
\text { solved } \\
(\mu \mathrm{g} / \mathrm{L} \text { as } \\
\mathrm{Zn})\end{array}$ \\
\hline
\end{tabular}

\begin{tabular}{|c|c|c|c|c|c|c|c|c|c|c|c|}
\hline \multicolumn{12}{|c|}{ CATARACT CREEK--Continued } \\
\hline \multirow[t]{18}{*}{44} & $10-16-96$ & $<.1$ & $<.1$ & $<1$ & 1 & $<1$ & $<1$ & $<1$ & 1 & 710 & 662 \\
\hline & $05-15-97$ & $<.1$ & -- & $<1$ & 2 & $<1$ & $<1$ & $<1$ & $<1$ & 450 & 321 \\
\hline & $07-23-97$ & -- & - & $<1$ & -- & 1 & -- & $<1$ & $<1$ & 370 & 294 \\
\hline & $09-24-97$ & -- & -- & $<1$ & - & 2 & - & $<1$ & $<1$ & 770 & 744 \\
\hline & $02-12-98$ & -- & - & $<1$ & -- & $<1$ & -- & $<1$ & $<1$ & 570 & 528 \\
\hline & $04-24-98$ & -- & -- & $<1$ & -- & $<1$ & -- & $<1$ & $<1$ & 290 & 231 \\
\hline & $05-06-98$ & -- & -- & $<1$ & - & $<1$ & -- & $<1$ & $<1$ & 140 & 120 \\
\hline & $06-26-98$ & -- & -- & $<1$ & - & $<1$ & -. & $<1$ & $<1$ & 170 & 135 \\
\hline & $07-21-98$ & -- & -- & $<1$ & -- & $<1$ & -- & $<1$ & $<1$ & 320 & 275 \\
\hline & $07-22-98$ & -- & -- & -- & -- & -- & -- & -- & -- & - & 406 \\
\hline & $07-23-98$ & -- & -- & - & - & -- & -- & - & -- & - & 376 \\
\hline & $07-24-98$ & -. & -- & -- & .. & -- & $\ldots$ & - & - & - & 433 \\
\hline & $09-22-98$ & -- & -- & $<1$ & -- & $<1$ & -- & $<1$ & $<1$ & 860 & 813 \\
\hline & $05-24-99$ & -- & -- & -- & -- & -- & -- & -- & -- & 380 & 178 \\
\hline & $08-02-99$ & -- & -- & -- & -- & -- & -- & -- & -- & -- & 714 \\
\hline & 08-03-99 & -- & -- & -- & -- & -- & -- & -- & -- & - & 570 \\
\hline & 08-04-99 & -- & -- & -- & -- & -- & -- & -- & -- & 770 & 727 \\
\hline & $08-05-99$ & -- & -- & -- & -- & -- & -- & -- & - & - & 515 \\
\hline \multirow[t]{2}{*}{45} & $10-16-96$ & -- & -- & -- & -- & $\sim$ & -- & -- & -- & 20 & -- \\
\hline & $05-13-97$ & -- & - & -- & -- & - & -- & -- & -- & 30 & - \\
\hline \multirow[t]{2}{*}{46} & $10-16-96$ & -- & -. & -- & -- & -. & -- & -. & -- & $<10$ & -- \\
\hline & $05-13-97$ & -- & -- & -- & -- & - & -- & -- & - & 20 & - \\
\hline \multirow[t]{19}{*}{47} & $10-15-96$ & $<.1$ & $<.1$ & $<1$ & $<1$ & $<1$ & $<1$ & $<1$ & 3 & 430 & 406 \\
\hline & $12-04-96$ & $<.1$ & $<.1$ & 1 & $<1$ & $<1$ & $<1$ & $<1$ & 2 & 350 & 320 \\
\hline & $02-11-97$ & -- & -- & 1 & - & 2 & -- & $<1$ & 2 & 340 & 324 \\
\hline & $04-18-97$ & -- & -- & $<1$ & -- & 1 & -- & $<1$ & 2 & 290 & 258 \\
\hline & $05-08-97$ & $\cdots$ & - & $<1$ & -- & $<1$ & -- & $<1$ & $<1$ & 230 & 181 \\
\hline & $05-15-97$ & $<.1$ & - & $<1$ & 1 & $<1$ & $<1$ & $<1$ & $<1$ & 240 & 132 \\
\hline & $05-22-97$ & $<.1$ & - & $<1$ & $<1$ & $<1$ & $<1$ & $<1$ & $<1$ & 160 & 139 \\
\hline & $06-03-97$ & -- & -- & $<1$ & -- & $<1$ & -- & $<1$ & $<1$ & 140 & 126 \\
\hline & $06-11-97$ & -- & - & $<1$ & -- & $<1$ & - & $<1$ & $<1$ & 160 & 102 \\
\hline & $06-17-97$ & -- & -- & $<1$ & -- & $<1$ & -- & $<1$ & $<1$ & 170 & 143 \\
\hline & $07-18-97$ & -- & -- & $<1$ & -- & $<1$ & - & $<1$ & $<1$ & 220 & 191 \\
\hline & $09-24-97$ & -- & -- & $<1$ & - & 1 & -- & $<1$ & 2 & 420 & 397 \\
\hline & $02-09-98$ & -- & -- & 1 & - & $<1$ & -- & $<1$ & 3 & 290 & 284 \\
\hline & $04-24-98$ & -- & -- & $<1$ & -- & $<1$ & -- & $<1$ & $<1$ & 290 & 207 \\
\hline & $05-05-98$ & -- & -- & $<1$ & - & $<1$ & -- & $<1$ & $<1$ & 170 & 131 \\
\hline & $09-22-98$ & -- & -- & 1 & - & $<1$ & -- & $<1$ & 2 & 470 & 467 \\
\hline & $05-25-99$ & -- & -- & -- & - & -- & -- & - & -- & 180 & 132 \\
\hline & $09-14-99$ & -- & -- & -- & - & -- & -- & -- & - & 480 & 488 \\
\hline & \multicolumn{11}{|c|}{ HIGH ORE CREEK } \\
\hline \multirow[t]{4}{*}{49} & $10-17-96$ & $<.1$ & $<.1$ & $<1$ & $<1$ & $<1$ & $<1$ & $<1$ & $<1$ & $<10$ & 4 \\
\hline & $05-09-97$ & $<.1$ & $<.1$ & $<1$ & $<1$ & $<1$ & $<1$ & $<1$ & $<1$ & $<10$ & 2 \\
\hline & $09-23-98$ & -- & - & $<1$ & - & $<1$ & -- & $<1$ & $<1$ & $<10$ & $<1$ \\
\hline & $09-15-99$ & -- & - & -- & - & - & -- & -- & -- & $<31$ & $<1$ \\
\hline \multirow[t]{2}{*}{50} & $10-17-96$ & -- & -- & -- & - & - & -- & -- & - & 180 & -- \\
\hline & $05-08-97$ & -- & -- & -- & - & -. & -- & -- & -- & 20 & -- \\
\hline
\end{tabular}


Table 2. Water-quality data for streams in the Boulder River watershed, Montana, 1996-99 (Continued)

\begin{tabular}{|c|c|c|c|c|c|c|c|c|c|c|}
\hline $\begin{array}{c}\text { Site } \\
\text { num- } \\
\text { ber } \\
\text { (fig. 2) }\end{array}$ & Station name & Date & Time & $\begin{array}{c}\text { Dis- } \\
\text { charge, } \\
\text { instanta- } \\
\text { neous } \\
\left(\mathrm{ft}^{3} / \mathrm{s}\right)\end{array}$ & $\begin{array}{c}\text { Sedi- } \\
\text { ment, } \\
\text { sus- } \\
\text { pended } \\
\text { (mg/L) }\end{array}$ & $\begin{array}{c}\text { Sedi- } \\
\text { ment, } \\
\text { sus- } \\
\text { pended, } \\
\text { diameter } \\
\text { (percent } \\
\text { finer } \\
\text { than } \\
0.062 \\
\text { mm) }\end{array}$ & $\begin{array}{c}\text { Spe- } \\
\text { cific } \\
\text { con- } \\
\text { duct- } \\
\text { ance, } \\
\text { field } \\
(\mu \mathrm{S} / \mathrm{cm})\end{array}$ & $\begin{array}{c}\text { pH, } \\
\text { field } \\
\text { (stan- } \\
\text { dard } \\
\text { units) }\end{array}$ & $\begin{array}{l}\text { Tem- } \\
\text { pera- } \\
\text { ture, } \\
\text { water } \\
\left({ }^{\circ} \mathrm{C}\right)\end{array}$ & $\begin{array}{c}\text { Hard- } \\
\text { ness } \\
\text { (mg/L } \\
\text { as } \\
\left.\mathrm{CaCO}_{3}\right)\end{array}$ \\
\hline \multicolumn{11}{|c|}{ HIGH ORE CREEK-Continued } \\
\hline \multirow[t]{2}{*}{51} & Comet Mine outflow near Basin & $05-08-97$ & 1530 & .33 & -- & - & 1,500 & - & 9.5 & -- \\
\hline & & 06-03-99 & 1130 & .20 & -- & -- & 1,140 & 7.4 & 11.0 & 615 \\
\hline \multirow[t]{6}{*}{52} & High Ore Creek below Comet Mine outflow, & $10-17-96$ & 1502 & $<1$ & 36 & 80 & 430 & 7.6 & 1.5 & 180 \\
\hline & near Basin & 05-09-97 & 1125 & 2.5 & 8 & 74 & 234 & 7.8 & 9.5 & 100 \\
\hline & & $05-22-97$ & 1145 & 3.9 & 18 & 70 & 192 & 7.9 & 11.5 & 76 \\
\hline & & 08-03-99 & 1800 & .36 & 7 & 77 & 300 & 8.2 & 20.0 & 140 \\
\hline & & $08-04-99$ & 1600 & -- & -- & -- & -- & -- & -- & -- \\
\hline & & $08-05-99$ & 1400 & -- & -- & -- & -- & -- & - & -- \\
\hline \multirow[t]{5}{*}{53} & High Ore Creek above Bishop Creek, near Basin & $10-17-96$ & 1600 & -- & 37 & 73 & 397 & 7.8 & 1.0 & 170 \\
\hline & & $05-09-97$ & 1305 & 3.2 & 16 & 50 & 232 & 8.0 & 11.5 & 100 \\
\hline & & $03-25-99$ & 1445 & 2.3 & 86 & 80 & 306 & 8.2 & 1.5 & 134 \\
\hline & & 06-03-99 & 1330 & 3.8 & 21 & 60 & 191 & 8.0 & 10.5 & 83 \\
\hline & & $09-15-99$ & 0900 & .34 & 6 & 71 & 328 & 8.2 & 6.5 & 150 \\
\hline \multirow[t]{2}{*}{54} & Bishop Creek near Basin & $10-17-96$ & 1600 & - & -- & -- & 275 & -- & 1.5 & -- \\
\hline & & $05-08-97$ & 1445 & 2.7 & -- & -- & 184 & -- & 10.0 & -- \\
\hline \multirow[t]{2}{*}{55} & Peters Gulch near Basin & $10-17-96$ & 1655 & .10 & - & -- & 324 & -- & 1.5 & -- \\
\hline & & $05-08-97$ & 1530 & .38 & - & -. & 288 & -- & 8.0 & -- \\
\hline \multirow[t]{26}{*}{56} & High Ore Creek near Basin & $10-15-96$ & 1235 & 1.2 & 5 & 73 & 368 & 8.2 & 5.5 & 170 \\
\hline & & $12-03-96$ & 1430 & 1.0 & 8 & 62 & 340 & 7.9 & 0.0 & 160 \\
\hline & & $02-12-97$ & 0920 & .89 & 8 & 81 & 334 & 7.6 & 0.0 & 150 \\
\hline & & $04-19-97$ & 1215 & 3.0 & 38 & 63 & 227 & 8.2 & 5.5 & 110 \\
\hline & & $05-09-97$ & 1500 & 5.4 & 40 & 54 & 231 & 8.4 & 14.0 & 96 \\
\hline & & $05-14-97$ & 1630 & -- & 77 & 82 & 217 & 8.1 & 15.5 & 89 \\
\hline & & $05-22-97$ & 1345 & 6.2 & 51 & 48 & 189 & 8.1 & 12.5 & 74 \\
\hline & & $06-03-97$ & 1330 & 6.8 & 32 & 59 & 182 & 8.2 & 14.0 & 80 \\
\hline & & $06-11-97$ & 1415 & 6.3 & 82 & 47 & 216 & 8.1 & 11.5 & 93 \\
\hline & & $06-17-97$ & 1400 & 5.7 & 29 & 43 & 210 & 8.2 & 16.0 & 95 \\
\hline & & $07-18-97$ & 0900 & 2.6 & 11 & 66 & 324 & 8.3 & 11.0 & 150 \\
\hline & & $09-23-97$ & 1300 & 1.3 & 8 & 60 & 336 & 8.3 & 10.5 & 160 \\
\hline & & $02-09-98$ & 1030 & 1.1 & 3 & 85 & 335 & 7.9 & 0.0 & 150 \\
\hline & & $04-24-98$ & 1515 & 3.5 & -- & -- & 225 & 8.2 & 7.0 & 98 \\
\hline & & $05-05-98$ & 1430 & 3.3 & 10 & 64 & 220 & 8.2 & -- & 97 \\
\hline & & $07-21-98$ & 1430 & 1.8 & 3 & 73 & 285 & 8.3 & 20.5 & 130 \\
\hline & & $07-22-98$ & 0950 & - & - & -- & 300 & 8.1 & 11.5 & -- \\
\hline & & $07-23-98$ & 0905 & -- & -- & -- & 302 & 8.2 & 12.0 & -- \\
\hline & & $07-24-98$ & 0920 & -- & -- & -- & 301 & 8.3 & 14.0 & - \\
\hline & & $09-23-98$ & 1200 & 1.0 & 5 & 73 & 330 & 8.3 & 8.0 & 160 \\
\hline & & $03-15-99$ & 1435 & 2.3 & 81 & 78 & 258 & 8.4 & 0.0 & 117 \\
\hline & & $03-25-99$ & 1620 & 4.6 & 308 & 49 & 288 & 8.2 & 17.0 & 130 \\
\hline & & $05-25-99$ & 1410 & 3.7 & 34 & 49 & 186 & 8.2 & 17.5 & 80 \\
\hline & & $06-03-99$ & 1500 & 5.4 & 56 & 61 & 186 & 8.0 & 11.0 & 82 \\
\hline & & $08-02-99$ & 1701 & .52 & 1 & 50 & 298 & 8.4 & 21.0 & 140 \\
\hline & & 09-14-99 & 1330 & .82 & 14 & 58 & 327 & 8.2 & 12.5 & 150 \\
\hline
\end{tabular}


Table 2. Water-quality data for streams in the Boulder River watershed, Montana, 1996-99 (Continued)

\begin{tabular}{|c|c|c|c|c|c|c|c|c|c|c|c|}
\hline $\begin{array}{c}\text { Site } \\
\text { num- } \\
\text { ber } \\
\text { (fig. 2) }\end{array}$ & Date & $\begin{array}{c}\text { Cal- } \\
\text { cium, } \\
\text { dissolved } \\
(\mathrm{mg} / \mathrm{L} \\
\text { as Ca) }\end{array}$ & $\begin{array}{l}\text { Magne- } \\
\text { sium, } \\
\text { dissolved } \\
\text { (mg/L } \\
\text { as } \mathbf{M g})\end{array}$ & $\begin{array}{c}\text { Sodium, } \\
\text { dissolved } \\
\text { (mg/L } \\
\text { as } \mathrm{Na} \text { ) }\end{array}$ & $\begin{array}{l}\text { Potas- } \\
\text { sium, } \\
\text { dissolved } \\
\text { (mg/L } \\
\text { as K) }\end{array}$ & $\begin{array}{c}\text { ANC, } \\
\text { lab } \\
(\mathrm{mg} / \mathrm{L} \\
\left.\text { as } \mathrm{CaCO}_{3}\right)\end{array}$ & $\begin{array}{c}\text { Sulfate, } \\
\text { dissolved } \\
(\mathrm{mg} / \mathrm{L} \\
\left.\text { as } \mathrm{SO}_{4}\right)\end{array}$ & $\begin{array}{c}\text { Chloride, } \\
\text { dissolved } \\
(\mathrm{mg} / \mathrm{L} \\
\text { as } \mathrm{Cl})\end{array}$ & $\begin{array}{c}\text { Fluoride, } \\
\text { dissolved } \\
\text { (mg/L } \\
\text { as F) }\end{array}$ & $\begin{array}{c}\text { Silica, } \\
\text { dissolved } \\
(\mathrm{mg} / \mathrm{L} \\
\left.\text { as } \mathrm{SiO}_{2}\right)\end{array}$ & $\begin{array}{c}\text { Dis- } \\
\text { solved } \\
\text { solids, } \\
\text { calcu- } \\
\text { lated } \\
(\mathrm{mg} / \mathrm{L})\end{array}$ \\
\hline
\end{tabular}

\begin{tabular}{|c|c|c|c|c|c|c|c|c|c|c|c|}
\hline \multicolumn{12}{|c|}{ HIGH ORE CREEK-Continued } \\
\hline \multirow[t]{2}{*}{51} & $05-08-97$ & -- & -- & -- & - & -- & -- & - & -- & -- & -- \\
\hline & 06-03-99 & 167 & 48 & 6.9 & 3.2 & 80 & 559 & 1.5 & -- & - & -- \\
\hline \multirow[t]{6}{*}{52} & $10-17-96$ & 50 & 14 & 4.7 & 1.3 & 66 & 140 & 1.3 & .10 & 17 & 273 \\
\hline & $05-09-97$ & 30 & 7.1 & 3.8 & 1.1 & 50 & 63 & .89 & $<.10$ & 16 & 154 \\
\hline & $05-22-97$ & 22 & 5.3 & 3.3 & .83 & 40 & 46 & .64 & $<.10$ & 16 & 120 \\
\hline & 08-03-99 & 38 & 9.6 & 5.3 & 1.8 & 65 & 82 & 1.4 & -- & -- & -- \\
\hline & 08-04-99 & -- & -- & -- & -- & -- & -- & - & -- & -- & - \\
\hline & $08-05-99$ & -- & -- & -- & - & - & -- & - & -- & - & -- \\
\hline \multirow[t]{5}{*}{53} & $10-17-96$ & 46 & 13 & 4.7 & 1.3 & 68 & 120 & 1.1 & .10 & 18 & 250 \\
\hline & $05-09-97$ & 30 & 7.1 & 4.3 & 1.1 & 53 & 58 & 1.1 & $<.10$ & 17 & 153 \\
\hline & $03-25-99$ & 38 & 10 & 3.6 & 1.5 & 38 & 101 & .57 & $<.10$ & 14 & 198 \\
\hline & 06-03-99 & 24 & 5.7 & 3.6 & 1.0 & 44 & 47 & .77 & -- & -- & -. \\
\hline & $09-15-99$ & 41 & 11 & 5.1 & 1.5 & 75 & 86 & .40 & -- & -- & - \\
\hline \multirow[t]{2}{*}{54} & $10-17-96$ & -- & -- & -- & -- & -- & -- & -- & -- & -- & -- \\
\hline & $05-08-97$ & -- & -- & - & - & -- & -- & -- & -- &.- & - \\
\hline \multirow[t]{2}{*}{55} & $10-17-96$ & -- & -- & -- & -- & -- & -. & -- & -- & -- & - \\
\hline & $05-08-97$ & - & -- & -- & -. & -- & -- & -- & -- & - & - \\
\hline \multirow[t]{26}{*}{56} & $10-15-96$ & 46 & 13 & 5.4 & 1.7 & 95 & 92 & 1.6 & .10 & 19 & 239 \\
\hline & $12-03-96$ & 43 & 12 & 5.5 & 1.6 & 86 & 88 & 1.4 & .10 & 19 & 226 \\
\hline & $02-12-97$ & 41 & 12 & -- & -- & -- & - & -- & - & -- & -- \\
\hline & $04-19-97$ & 30 & 7.8 & 4.3 & 1.5 & 66 & 58 & 1.4 & $<.10$ & 17 & 161 \\
\hline & $05-09-97$ & 27 & 6.9 & 4.5 & 1.4 & 60 & 50 & 1.4 & .12 & 17 & 147 \\
\hline & $05-14-97$ & 25 & 6.4 & 4.2 & 1.3 & 50 & 48 & 1.1 & .13 & 17 & 135 \\
\hline & $05-22-97$ & 21 & 5.4 & 3.8 & 1.1 & 49 & 35 & .88 & $<.10$ & 17 & 115 \\
\hline & $06-03-97$ & 22 & 5.8 & 3.9 & 1.1 & 51 & 35 & .95 & .11 & 17 & 118 \\
\hline & $06-11-97$ & 26 & 6.8 & 4.2 & 1.1 & 58 & 43 & .74 & .10 & 17 & 135 \\
\hline & $06-17-97$ & 27 & 6.8 & 4.3 & 1.1 & 60 & 40 & .77 & .11 & 18 & 135 \\
\hline & $07-18-97$ & 40 & 11 & 5.2 & 1.5 & 88 & 73 & 1.2 & .17 & 19 & 207 \\
\hline & 09-23-97 & 43 & 13 & 5.9 & 1.6 & 98 & 71 & 1.6 & .17 & 19 & 217 \\
\hline & $02-09-98$ & 42 & 12 & 5.4 & 1.6 & 90 & 77 & 1.6 & .12 & 18 & 215 \\
\hline & $04-24-98$ & 28 & 6.7 & 4.3 & 1.4 & 61 & 45 & 1.5 & $<.10$ & 15 & 140 \\
\hline & $05-05-98$ & 27 & 6.9 & 4.3 & 1.3 & 60 & 44 & 1.1 & .12 & 16 & 137 \\
\hline & $07-21-98$ & 37 & 9.7 & 5.4 & 1.7 & 87 & 55 & 1.0 & .14 & 20 & 182 \\
\hline & $07-22-98$ & -- & -- & -- & -. & -- & -- & -- & - & -- & -- \\
\hline & $07-23-98$ & -- & -- & -- & -- & -- & -- & -- & - & -- & - \\
\hline & $07-24-98$ & -- & -- & -- & -- & -- & -- & -- & - & -- & - \\
\hline & $09-23-98$ & 44 & 13 & 5.8 & 1.7 & 101 & 76 & 1.7 & .14 & 19 & 224 \\
\hline & $03-15-99$ & 32 & 9.0 & 4.2 & 2.3 & 65 & 59 & 1.3 & $<.10$ & 15 & 162 \\
\hline & $03-25-99$ & 36 & 9.7 & 4.5 & 1.7 & 60 & 74 & 1.2 & .11 & 16 & 182 \\
\hline & $05-25-99$ & 23 & 5.7 & 3.9 & 1.2 & 56 & 33 & .91 & - & -- & - \\
\hline & 06-03-99 & 23 & 5.9 & 3.9 & 1.1 & 50 & 39 & 1.0 & -- & -- & -- \\
\hline & 08-02-99 & 38 & 11 & 5.8 & 2.0 & 90 & 59 & 2.2 & - & -- & - \\
\hline & $09-14-99$ & 42 & 12 & 5.8 & 1.9 & 97 & 68 & 1.3 & - & -- & -- \\
\hline
\end{tabular}


Table 2. Water-quality data for streams in the Boulder River watershed, Montana, 1996-99 (Continued)

\begin{tabular}{|c|c|c|c|c|c|c|c|}
\hline $\begin{array}{c}\text { Site } \\
\text { num- } \\
\text { ber } \\
\text { (fig. 2) }\end{array}$ & Date & $\begin{array}{c}\text { Nitrite, } \\
\text { dissolved } \\
\text { (mg/L } \\
\text { as } \mathbf{N})\end{array}$ & $\begin{array}{l}\text { Nitrite } \\
\text { plus } \\
\text { nitrate, } \\
\text { dissolved } \\
\text { (mg/L } \\
\text { as } N \text { ) }\end{array}$ & $\begin{array}{c}\text { Ammonia, } \\
\text { dissolved } \\
\text { (mg/L } \\
\text { as } N)\end{array}$ & $\begin{array}{c}\text { Ammonia plus } \\
\text { organic } \\
\text { nitrogen, } \\
\text { total } \\
\text { (mg/L } \\
\text { as } N)\end{array}$ & $\begin{array}{c}\text { Phos- } \\
\text { phorus, } \\
\text { total } \\
\text { (mg/L } \\
\text { as P) }\end{array}$ & $\begin{array}{l}\text { Phos- } \\
\text { phorus, } \\
\text { ortho- } \\
\text { phosphate, } \\
\text { dissolved } \\
\text { (mg/L } \\
\text { as P) }\end{array}$ \\
\hline
\end{tabular}

\begin{tabular}{|c|c|c|c|c|c|c|c|}
\hline \multicolumn{8}{|c|}{ HIGH ORE CREEK--Continued } \\
\hline \multirow[t]{2}{*}{51} & $05-08-97$ & -- & -- & -- & -- & -- & -- \\
\hline & $06-03-99$ & -- & -- & -- & - & -- & -- \\
\hline \multirow[t]{6}{*}{52} & $10-17-96$ & -- & -- & - & -- & - & -- \\
\hline & $05-09-97$ & -- & -- & -- & -- & - & -- \\
\hline & $05-22-97$ & -- & -- & -- & - & -- & -- \\
\hline & 08-03-99 & - & -- & -- & -- & -- & -- \\
\hline & 08-04-99 & -- & -- & - & -- & -- & -- \\
\hline & $08-05-99$ & -- & -- & -- & -- & - & -- \\
\hline \multirow[t]{5}{*}{53} & $10-17-96$ & -- & -- & -- & -- & -- & $-\cdot$ \\
\hline & $05-09-97$ & -- & -- & -- & - & -- & -- \\
\hline & $03-25-99$ & -- & -- & -- & -- & -- & -- \\
\hline & 06-03-99 & -- & -- & -- & -- & -- & -- \\
\hline & $09-15-99$ & -- & -- & -- & -- & -- & -- \\
\hline \multirow[t]{2}{*}{54} & $10-17-96$ & -- & -- & -- & -- & -- & -- \\
\hline & $05-08-97$ & -- & -- & -- & -- & -- & -- \\
\hline \multirow[t]{2}{*}{55} & $10-17-96$ & -- & -- & -- & - & -- & -- \\
\hline & $05-08-97$ & -- & -- & - & -- & - & $\cdots$ \\
\hline \multirow[t]{26}{*}{56} & $10-15-96$ & -- & -- & -- & -- & -- & -- \\
\hline & $12-03-96$ & $<.01$ & .15 & .040 & -- & -- & .020 \\
\hline & $02-12-97$ & - & - & -- & -- & -- & -- \\
\hline & $04-19-97$ & -- & -- & -- & -- & -- & -- \\
\hline & $05-09-97$ & $<.01$ & .12 & $<.015$ & -- & -- & $<.010$ \\
\hline & $05-14-97$ & $<.01$ & .06 & $<.015$ & -- & -- & $<.010$ \\
\hline & $05-22-97$ & $<.01$ & $<.05$ & $<.015$ & -- & -- & $<.010$ \\
\hline & $06-03-97$ & - & -- & -- & -- & -- & -- \\
\hline & $06-11-97$ & -- & -- & -- & -- & -- & -- \\
\hline & $06-17-97$ & -- & -- & -- & -- & - & -- \\
\hline & $07-18-97$ & $<.01$ & $<.05$ & $<.015$ & -- & -- & .010 \\
\hline & $09-23-97$ & $<.01$ & $<.05$ & $<.015$ & $<.2$ & $<.010$ & $<.010$ \\
\hline & $02-09-98$ & $<.01$ & .17 & .032 & -- & -- & .016 \\
\hline & $04-24-98$ & -- & -- & -- & -- & -- & -- \\
\hline & $05-05-98$ & - & -- & -- & -- & -- & -- \\
\hline & $07-21-98$ & $<.01$ & $<.05$ & .034 & -- & - & $<.010$ \\
\hline & $07-22-98$ & - & - & -- & -- & -- & -- \\
\hline & $07-23-98$ & -- & -- & -- & -- & -- & -- \\
\hline & $07-24-98$ & -- & - & -- & - & -- & -- \\
\hline & $09-23-98$ & - & -- & -- & -- & -- & -- \\
\hline & $03-15-99$ & - & - & -- & - & -- & -- \\
\hline & $03-25-99$ & -- & -- & -- & -- & -- & -- \\
\hline & $05-25-99$ & -- & -- & -- & -- & -. & -- \\
\hline & $06-03-99$ & -- & -- & -- & -- & -- & -- \\
\hline & $08-02-99$ & -- & - & -- & -- & -- & -- \\
\hline & 09-14-99 & - & - & -- & -- & - & -- \\
\hline
\end{tabular}


Table 2. Water-quality data for streams in the Boulder River watershed, Montana, 1996-99 (Continued)

\begin{tabular}{|c|c|c|c|c|c|c|c|c|c|c|c|c|}
\hline $\begin{array}{c}\text { Site } \\
\text { num- } \\
\text { ber } \\
\text { (fig. 2) }\end{array}$ & Date & $\begin{array}{l}\text { Alumi- } \\
\text { num, } \\
\text { total } \\
\text { recov- } \\
\text { erable } \\
(\mu \mathrm{g} / \mathrm{L} \text { as } \\
\text { Al) }\end{array}$ & $\begin{array}{l}\text { Alumi- } \\
\text { num, } \\
\text { dis- } \\
\text { solved } \\
(\mu \mathrm{g} / \mathrm{L} \text { as } \\
\text { Al) }\end{array}$ & $\begin{array}{l}\text { Anti- } \\
\text { mony, } \\
\text { dis- } \\
\text { solved } \\
(\mu \mathrm{g} / \mathrm{L} \text { as } \\
\text { Sb) }\end{array}$ & $\begin{array}{c}\text { Arsenic, } \\
\text { total } \\
\text { recov- } \\
\text { erable } \\
(\mu \mathrm{g} / \mathrm{L} \text { as } \\
\text { As) }\end{array}$ & $\begin{array}{c}\text { Arsenic, } \\
\text { dis- } \\
\text { solved } \\
(\mu \mathrm{g} / \mathrm{L} \text { as } \\
\text { As })\end{array}$ & $\begin{array}{c}\text { Barium, } \\
\text { dis- } \\
\text { solved } \\
(\mu \mathrm{g} / \mathrm{L} \text { as } \\
\mathrm{Ba})\end{array}$ & $\begin{array}{c}\text { Beryl- } \\
\text { lium, } \\
\text { dis- } \\
\text { solved } \\
(\mu \mathrm{g} / \mathrm{L} \text { as } \\
\text { Be })\end{array}$ & $\begin{array}{c}\text { Cad- } \\
\text { mium, } \\
\text { total } \\
\text { recov- } \\
\text { erable } \\
(\mu \mathrm{g} / \mathrm{L} \text { as } \\
\text { Cd) }\end{array}$ & $\begin{array}{c}\text { Cad- } \\
\text { mium, } \\
\text { dis- } \\
\text { solved } \\
(\mu \mathrm{g} / L \text { as } \\
\text { Cd })\end{array}$ & $\begin{array}{c}\text { Chro- } \\
\text { mium, } \\
\text { total } \\
\text { recov- } \\
\text { erable } \\
(\mu \mathrm{g} / \mathrm{L} \text { as } \\
\mathrm{Cr})\end{array}$ & $\begin{array}{c}\text { Chro- } \\
\text { mium, } \\
\text { dis- } \\
\text { solved } \\
(\mu \mathrm{g} / \mathrm{L} \text { as } \\
\text { Cr) }\end{array}$ \\
\hline \multicolumn{13}{|c|}{ HIGH ORE CREEK--Continued } \\
\hline \multirow[t]{2}{*}{51} & $05-08-97$ & - & - & -- & -- & -- & - & -- & - & -- & -- & -- \\
\hline & $06-03-99$ & e28 & 6.0 & -- & 43 & 16 & - & -- & 37 & 36 & - & -- \\
\hline \multirow[t]{6}{*}{52} & $10-17-96$ & -- & 2.5 & $<1$ & 110 & 8 & 25 & $<1$ & 6 & 4.6 & $<1$ & $<1$ \\
\hline & $05-09-97$ & -- & 39 & $<1$ & 25 & 5 & 24 & $<1$ & 5 & 4.6 & -- & $<1$ \\
\hline & $05-22-97$ & 220 & 6.3 & $<1$ & 41 & 4 & 20 & $<1$ & 3 & 2.4 & $<1$ & $<1$ \\
\hline & $08-03-99$ & 200 & 2.2 & $-\cdot$ & 43 & 23 & -- & -- & 3 & 1.7 & -- & -- \\
\hline & 08-04-99 & - & - & - & -- & -- & - & - & - & 2.7 & -- & -- \\
\hline & 08-05-99 & -- & -- & -- & -- & -- & -- & -- & -- & 2.5 & -- & -- \\
\hline \multirow[t]{5}{*}{53} & $10-17-96$ & - & 2.5 & 1 & 110 & 14 & 27 & $<1$ & 6 & 4.9 & $<1$ & $<1$ \\
\hline & $05-09-97$ & -- & 7.8 & 1 & 44 & 9 & 27 & $<1$ & 5 & 4.6 & -- & $<1$ \\
\hline & $03-25-99$ & 980 & 5.8 & $<1$ & 180 & 8 & 26 & $<1$ & 29 & 22 & -. & $<1$ \\
\hline & $06-03-99$ & 300 & 5.6 & - & 150 & 12 & -- & - & 5 & 3.4 & -- & - \\
\hline & $09-15-99$ & 90 & 5.7 & -- & 38 & 21 & -. & -- & 4 & 3.3 & -- & -- \\
\hline \multirow[t]{2}{*}{54} & $10-17-96$ & - & -- & -- & -- & -- & - & -- & - & -- & -- & -- \\
\hline & $05-08-97$ & - & -- & - & -- & -- & - & - & - & -- & - & - \\
\hline \multirow[t]{2}{*}{55} & $10-17-96$ & - & - & - & -- & -- & - & $\cdots$ & - & -- & -- & -- \\
\hline & $05-08-97$ & - & -- & -- & -. & -- & -- & -- & -- & - & -- & -- \\
\hline \multirow[t]{26}{*}{56} & $10-15-96$ & - & 1.5 & 2 & 28 & 18 & 35 & $<1$ & 5 & 4.4 & $<1$ & $<1$ \\
\hline & $12-03-96$ &.- & $<1$ & 2 & 39 & 14 & 32 & $<1$ & 5 & 4.5 & $<1$ & $<1$ \\
\hline & $02-12-97$ & - & 1.5 & 2 & 37 & 13 & 32 & $<1$ & 5 & 4.0 & - & $<1$ \\
\hline & $04-19-97$ & - & 7.0 & 1 & 75 & 10 & 27 & $<1$ & 7 & 4.4 & -- & 1 \\
\hline & $05-09-97$ & -- & 63 & 1 & 64 & 13 & 31 & $<1$ & 4 & 2.5 & $<1$ & 1 \\
\hline & $05-14-97$ & 1,200 & 4.4 & 1 & 200 & 13 & 27 & $<1$ & 7 & 2.3 & 1 & $<1$ \\
\hline & $05-22-97$ & 650 & 5.8 & 1 & 69 & 9 & 25 & $<1$ & 3 & - & $<1$ & $<1$ \\
\hline & $06-03-97$ & 390 & 5.7 & 1 & 44 & 10 & 26 & $<1$ & 3 & 1.9 & -. & $<1$ \\
\hline & $06-11-97$ & 950 & 6.3 & 1 & 130 & 14 & 31 & $<1$ & 5 & 2.4 & -- & $<1$ \\
\hline & $06-17-97$ & 190 & 3.6 & 1 & 33 & 12 & 30 & $<1$ & 3 & 2.3 & -- & $<1$ \\
\hline & $07-18-97$ & - & 1.1 & 2 & 31 & 13 & 39 & $<1$ & 5 & 4.7 & - & 1 \\
\hline & $09-23-97$ & 50 & $<1.0$ & 2 & 25 & 14 & 35 & $<1$ & 4 & 3.8 & .. & 1 \\
\hline & $02-09-98$ & 40 & $<1.0$ & 2 & 19 & 15 & 31 & $<1$ & 4 & 4.2 & -- & 1 \\
\hline & $04-24-98$ & 370 & 4.3 & 1 & 58 & 10 & 26 & $<1$ & 5 & 2.8 & -- & $<1$ \\
\hline & $05-05-98$ & 90 & 3.6 & 1 & 18 & 12 & 27 & $<1$ & 3 & 2.3 & -- & $<1$ \\
\hline & $07-21-98$ & 50 & 2.6 & 2 & 24 & 22 & 36 & $<1$ & 2 & 2.4 & -- & $<1$ \\
\hline & $07-22-98$ & -- & -- & -- & -- & - & -- & -- & - & 3.7 & - & - \\
\hline & $07-23-98$ & -- & -- & -- & -- & -- & -- & -- & - & 3.7 & - & - \\
\hline & $07-24-98$ & -- & -- & -- & -- & -- & -- & -- & -- & 3.7 & -- & - \\
\hline & $09-23-98$ & 40 & $<1$ & 2 & -- & -- & 36 & $<1$ & 4 & 3.3 & -- & $<1$ \\
\hline & $03-15-99$ & 1,440 & 3.3 & 1 & 100 & 23 & 25 & $<1$ & 6 & 3.4 & -- & $<1$ \\
\hline & $03-25-99$ & 2,920 & 2.1 & 1 & 350 & 11 & 29 & $<1$ & 23 & 7.5 & -- & $<1$ \\
\hline & $05-25-99$ & 460 & 4.1 & -- & 57 & 15 & -. & -- & 3 & 1.6 & -- & -- \\
\hline & 06-03-99 & 34 & 7 & -. & 100 & 15 & -- & -- & 5 & 2.0 & -- & -- \\
\hline & $08-02-99$ & $<30$ & 1.5 & -- & 38 & 33 & -- & -- & 2 & 1.5 & - & - \\
\hline & $09-14-99$ & 250 & 3.9 & -- & 60 & 26 & -- & -- & 4 & 2.2 & -- & -- \\
\hline
\end{tabular}


Table 2. Water-quality data for streams in the Boulder River watershed, Montana, 1996-99 (Continued)

\begin{tabular}{|c|c|c|c|c|c|c|c|c|c|c|c|}
\hline $\begin{array}{c}\text { Site } \\
\text { num- } \\
\text { ber } \\
\text { (fig. 2) }\end{array}$ & Date & $\begin{array}{c}\text { Cobalt, } \\
\text { dis- } \\
\text { solved } \\
(\mu \mathrm{g} / \mathrm{L} \text { as } \\
\text { Co) }\end{array}$ & $\begin{array}{l}\text { Copper, } \\
\text { total recov- } \\
\text { erable } \\
(\mu \mathrm{g} / \mathrm{L} \text { as } \\
\mathrm{Cu})\end{array}$ & $\begin{array}{l}\text { Copper, } \\
\text { dis- } \\
\text { solved } \\
(\mu \mathrm{g} / \mathrm{L} \text { as } \\
\text { Cu })\end{array}$ & $\begin{array}{c}\text { Iron, } \\
\text { total } \\
\text { recov- } \\
\text { erable } \\
(\mu \mathrm{g} / \mathrm{L} \text { as } \\
\text { Fe) }\end{array}$ & $\begin{array}{c}\text { Iron, } \\
\text { dis- } \\
\text { solved } \\
(\mu \mathrm{g} / \mathrm{L} \text { as } \\
\text { Fe) }\end{array}$ & $\begin{array}{c}\text { Lead, } \\
\text { total } \\
\text { recov- } \\
\text { erable } \\
(\mu \mathrm{g} / \mathrm{L} \text { as } \\
\text { Pb) }\end{array}$ & $\begin{array}{c}\text { Lead, } \\
\text { dis- } \\
\text { solved } \\
(\mu \mathrm{g} / \mathrm{L} \text { as } \\
\text { Pb) }\end{array}$ & $\begin{array}{c}\text { Lithium, } \\
\text { dis- } \\
\text { solved } \\
(\mu \mathrm{g} / \mathrm{L} \text { as } \\
\mathrm{Li})\end{array}$ & $\begin{array}{l}\text { Manga- } \\
\text { nese, } \\
\text { total } \\
\text { recov- } \\
\text { erable } \\
(\mu \mathrm{g} / \mathrm{L} \text { as }\end{array}$ & $\begin{array}{l}\text { Manga- } \\
\text { nese, } \\
\text { dis- } \\
\text { solved } \\
(\mu \mathrm{g} / \mathrm{L} \text { as } \\
\text { Mn) }\end{array}$ \\
\hline
\end{tabular}

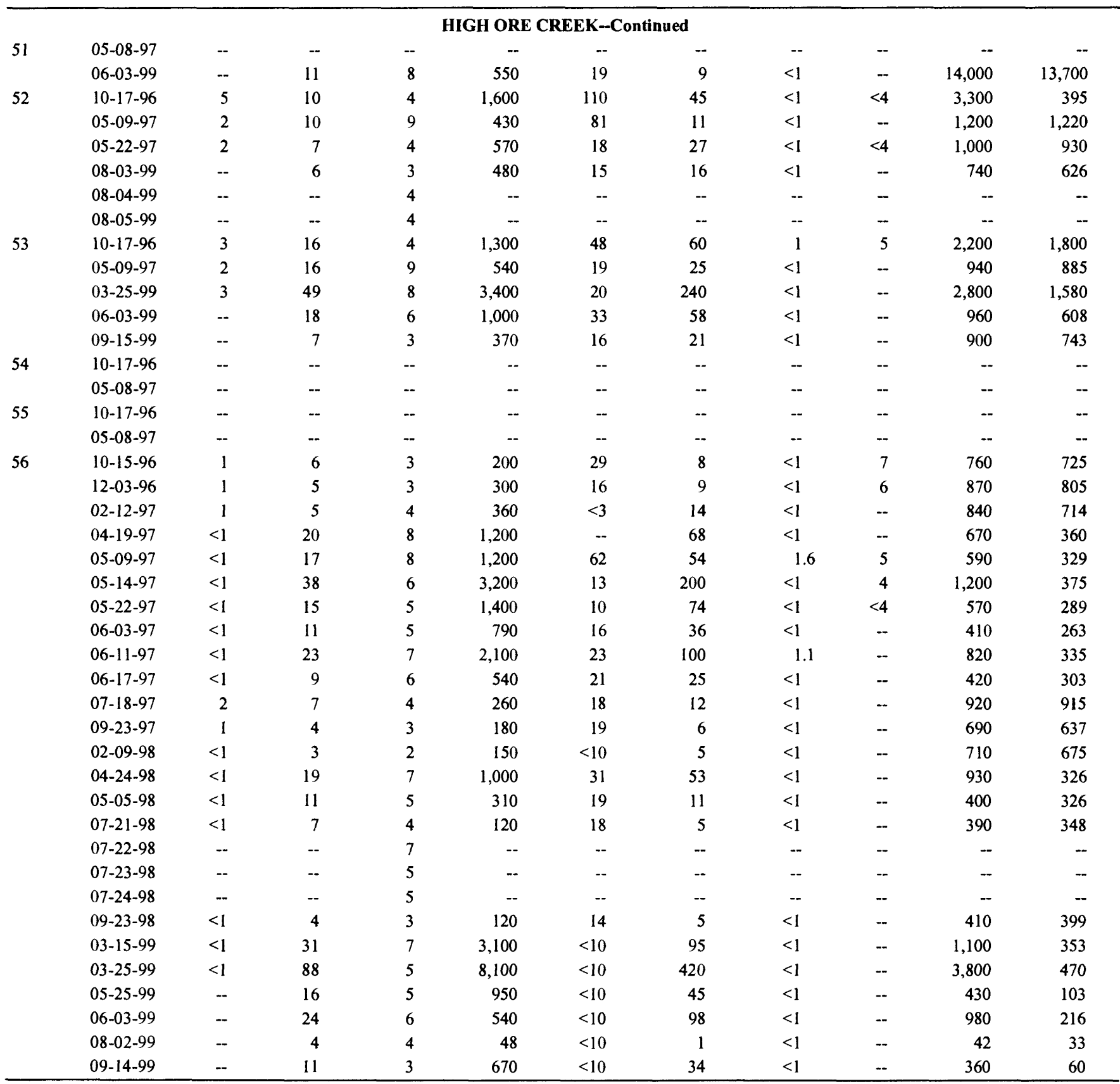


Table 2. Water-quality data for streams in the Boulder River watershed, Montana, 1996-99 (Continued)

\begin{tabular}{|c|c|c|c|c|c|c|c|c|c|c|c|}
\hline $\begin{array}{c}\text { Site } \\
\text { num- } \\
\text { ber } \\
\text { (fig. 2) }\end{array}$ & Date & $\begin{array}{c}\text { Mercury, } \\
\text { total } \\
\text { recov- } \\
\text { erable } \\
(\mu \mathrm{g} / \mathrm{L} \text { as } \\
\mathrm{Hg})\end{array}$ & $\begin{array}{c}\text { Mercury, } \\
\text { dis- } \\
\text { solved } \\
(\mu \mathrm{g} / \mathrm{L} \text { as } \\
\text { Hg) }\end{array}$ & $\begin{array}{c}\text { Molyb- } \\
\text { denum, } \\
\text { dis- } \\
\text { solved } \\
(\mu \mathrm{g} / \mathrm{L} \text { as } \\
\text { Mo })\end{array}$ & $\begin{array}{c}\text { Nickel, } \\
\text { total } \\
\text { recov- } \\
\text { erable } \\
(\mu \mathrm{g} / \mathrm{L} \text { as } \\
\mathrm{Ni})\end{array}$ & $\begin{array}{c}\text { Nickel, } \\
\text { dis- } \\
\text { solved } \\
(\mu \mathrm{g} / \mathrm{L} \text { as } \\
\mathrm{Ni})\end{array}$ & $\begin{array}{c}\text { Silver, } \\
\text { total } \\
\text { recov- } \\
\text { erable } \\
(\mu \mathrm{g} / \mathrm{L} \text { as } \\
\mathrm{Ag})\end{array}$ & $\begin{array}{l}\text { Silver, } \\
\text { dis- } \\
\text { solved } \\
(\mu \mathrm{g} / \mathrm{L} \text { as } \\
\text { Ag) }\end{array}$ & $\begin{array}{c}\text { Uranium, } \\
\text { dis- } \\
\text { solved } \\
(\mu \mathrm{g} / \mathrm{L} \text { as } \\
\mathrm{U})\end{array}$ & $\begin{array}{c}\text { Zinc, } \\
\text { total } \\
\text { recov- } \\
\text { erable } \\
(\mu \mathrm{g} / \mathrm{L} \text { as } \\
\mathrm{Zn})\end{array}$ & $\begin{array}{c}\text { Zinc, } \\
\text { dis- } \\
\text { solved } \\
(\mu \mathrm{g} / \mathrm{L} \text { as } \\
\mathrm{Zn})\end{array}$ \\
\hline
\end{tabular}

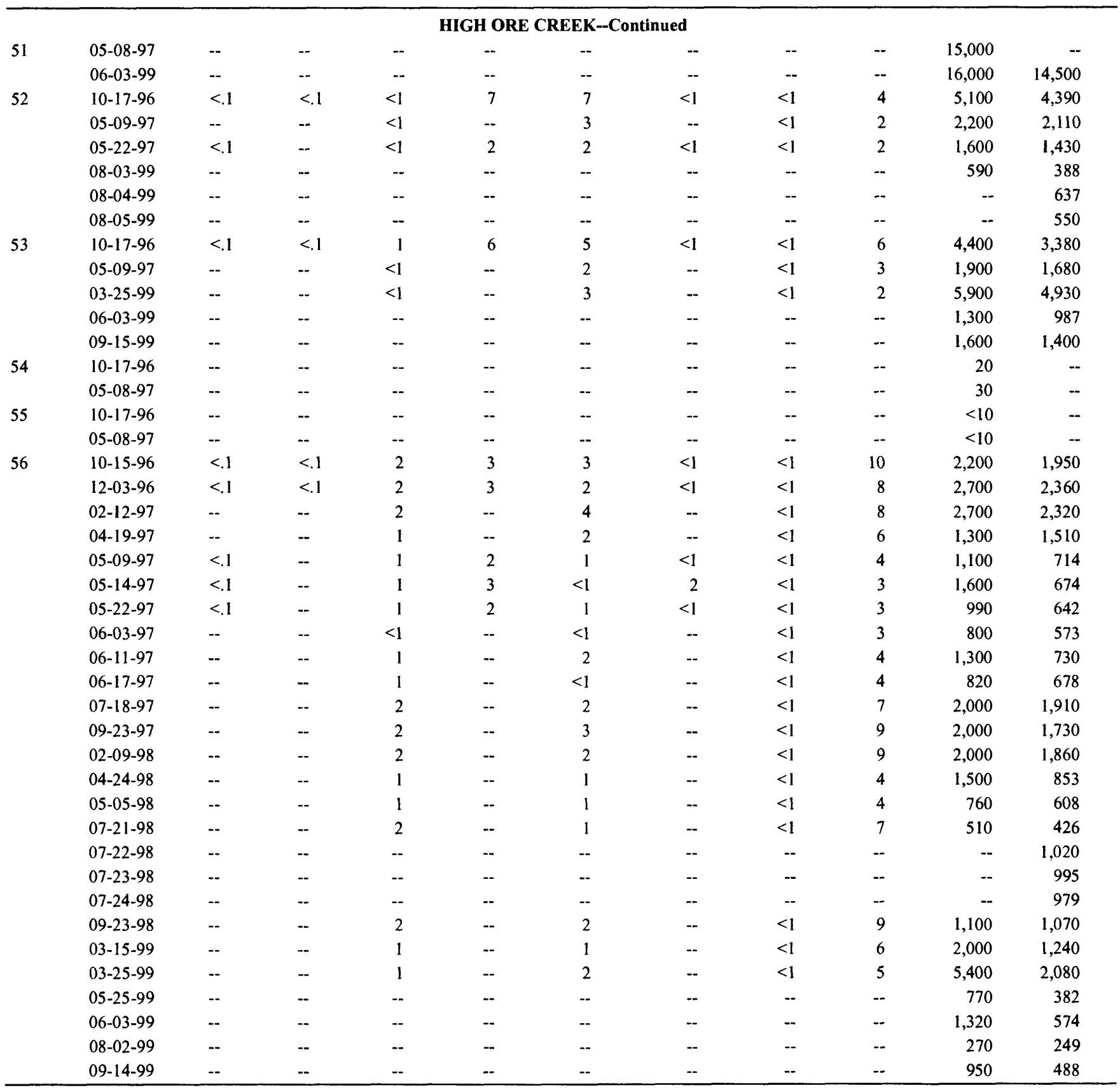


Table 3. Historical water-quality sampling sites in the Boulder River watershed, Montana, with data in the Montana Bureau of Mines and Geology Ground Water Information Center database

[Abbreviations: MBMG, Montana Bureau of Mines and Geology; USGS, U.S. Geological Survey; $\mathrm{ft}$, feet; mi, mile. Symbol: --, no data]

\begin{tabular}{|c|c|c|c|c|c|}
\hline $\begin{array}{c}\text { Site } \\
\text { number } \\
\text { (fig. 2) }\end{array}$ & $\begin{array}{c}\text { Analogous } \\
\text { USGS site } \\
\text { (fig. 2) }\end{array}$ & $\begin{array}{l}\text { MBMG site } \\
\text { number }\end{array}$ & Latitude & Longitude & Site name \\
\hline \multicolumn{6}{|c|}{ BOULDER RIVER } \\
\hline $1 \mathrm{M}$ & 3 & $M: 144336$ & 461611 & 1121643 & Boulder River above Kleinsmith Gulch \\
\hline $26 \mathrm{M}$ & & M:122779 & 461614 & 1121427 & Boulder River above Cataract Creek \\
\hline $68 \mathrm{M}$ & & $M: 121565$ & 461614 & 1121424 & Boulder River below Cataract Creek \\
\hline $69 \mathrm{M}$ & & M: 122777 & 461614 & 1121358 & Boulder River at old railsoad bridge \\
\hline $70 \mathrm{M}$ & 48 & $\mathrm{M}: 122778$ & 461611 & 1121338 & Boulder River 1 mi below Cataract Creek \\
\hline $71 \mathrm{M}$ & & M:122775 & 461552 & 1121309 & Boulder River 2 mi below Cataract Creek \\
\hline $82 \mathrm{M}$ & & M: 137080 & -- & -- & Boomerang Gulch 1 mi below Hope and Bullion Mine \\
\hline $83 \mathrm{M}$ & & M:137086 & -- & -- & Boomerang Gulch below Molly McGregor and Baltimore Mines \\
\hline \multicolumn{6}{|c|}{ BASIN CREEK } \\
\hline $2 \mathrm{M}$ & 5 & M:128487 & 462354 & 1121732 & Basin Creek above Buckeye Mine \\
\hline $3 \mathrm{M}$ & 8 & M: 128462 & 462340 & 1121756 & Basin Creek below Buckeye Mine \\
\hline $4 \mathrm{M}$ & & M:890860 & 462139 & 1122047 & Basin Creek below Weasel Gulch \\
\hline $5 \mathrm{M}$ & & M:128194 & 462109 & 1122046 & Basin Creek $0.5 \mathrm{mi}$ above Jack Creek \\
\hline $6 \mathrm{M}$ & & M: 128477 & 462247 & 1121638 & Jack Creek above Hawkeye Mine \\
\hline $7 \mathrm{M}$ & & M: 128478 & 462233 & 1121652 & Jack Creek below Hawkeye Mine \\
\hline $8 \mathrm{M}$ & & M:123696 & 462229 & 1121703 & Jack Creek below Hawkeye Mine \\
\hline $9 \mathrm{M}$ & & $M: 123713$ & 462227 & 1121635 & Jack Creek tributary $1.25 \mathrm{mi}$ above Bullion Mine tributary \\
\hline $10 \mathrm{M}$ & & $M: 123712$ & 462213 & 1121700 & Vindicator Mine tributary \\
\hline $11 \mathrm{M}$ & 16 & $\begin{array}{l}M: 128490 \\
M: 128501\end{array}$ & $\begin{array}{l}-- \\
--\end{array}$ & - & Jack Creek above Bullion Mine tributary \\
\hline $12 \mathrm{M}$ & & $M: 128468$ & -- & -- & Bullion Mine tributary above Bullion Mine \\
\hline $13 \mathrm{M}$ & & $\begin{array}{l}M: 128482 \\
M: 128494\end{array}$ & $\begin{array}{c}462131 \\
--\end{array}$ & $\begin{array}{c}1121749 \\
-\end{array}$ & Bullion Mine tributary below Bullion Mine \\
\hline $14 \mathrm{M}$ & 17 & $\begin{array}{l}M: 128504 \\
M: 128491\end{array}$ & $\begin{array}{c}462153 \\
--\end{array}$ & $\begin{array}{c}1121817 \\
--\end{array}$ & Bullion Mine tributary at mouth \\
\hline $15 \mathrm{M}$ & 19 & M: 128489 & 462152 & 1121833 & Jack Creek below Bullion Mine tributary \\
\hline $16 \mathrm{M}$ & & M: 123716 & 462137 & 1121857 & Jack Creek $0.5 \mathrm{mi}$ below Bullion Mine tributary \\
\hline $17 \mathrm{M}$ & & M: 123715 & 462137 & 1121908 & $\begin{array}{l}\text { Jack Creek tributary } 0.25 \mathrm{mi} \text { above Bullion Mine smelter } \\
\text { tributary }\end{array}$ \\
\hline $18 \mathrm{M}$ & & $\begin{array}{l}\text { M:123702 } \\
\text { M:128471 } \\
\text { M:128496 }\end{array}$ & $\begin{array}{c}462113 \\
-- \\
--\end{array}$ & $\begin{array}{c}1121816 \\
- \\
-\end{array}$ & Bullion Mine smelter tributary above smelter \\
\hline $19 \mathrm{M}$ & & $\begin{array}{l}M: 128492 \\
M: 128497\end{array}$ & $\begin{array}{l}-- \\
-\end{array}$ & - & Bullion Mine smelter tributary below smelter \\
\hline $20 \mathrm{M}$ & & M: 123717 & 462049 & 1122010 & Jack Creek $0.25 \mathrm{mi}$ above mouth \\
\hline $21 \mathrm{M}$ & & M: 128196 & 462046 & 1122018 & Jack Creek $0.125 \mathrm{mi}$ above mouth \\
\hline $22 \mathrm{M}$ & & $M: 890861$ & 462033 & 1122012 & Basin Creek below Jack Creek \\
\hline $23 \mathrm{M}$ & & $M: 128195$ & 462039 & 1122022 & Basin Creek above Lily-of-the-West Gulch \\
\hline $24 M$ & & $\mathrm{M}: 128511$ & 461740 & 1121645 & Lily-of-the-West Gulch \\
\hline $25 \mathrm{M}$ & & M:128197 & 461709 & 1121632 & Basin Creek above Spring Gulch \\
\hline \multicolumn{6}{|c|}{ CATARACT CREEK } \\
\hline $27 \mathrm{M}$ & & M:134779 & 462243 & 1121413 & Overland Creek $20 \mathrm{ft}$ above west adit \\
\hline $28 \mathrm{M}$ & & M:134782 & 462241 & 1121408 & Overland Creek $100 \mathrm{ft}$ below east shaft \\
\hline $29 \mathrm{M}$ & & M: 132718 & 462140 & 1121514 & Rocker Creek $10 \mathrm{ft}$ above seep entrance \\
\hline $30 \mathrm{M}$ & & M: 132720 & 462143 & 1121510 & Rocker Creek $10 \mathrm{ft}$ below west adit discharge \\
\hline $31 \mathrm{M}$ & & $\mathrm{M}: 132719$ & 462143 & 1121502 & Rocker Creek below Ada Mine site \\
\hline $32 \mathrm{M}$ & & M:128549 & 462122 & 1121050 & Hoodoo Creek below Occidental/Blue Diamond Mine \\
\hline $33 \mathrm{M}$ & 36 & $\begin{array}{l}M: 121539 \\
M: 128538\end{array}$ & - & -- & Cataract Creek at unnamed mine \#1 \\
\hline $34 \mathrm{M}$ & & M: 128456 & 462018 & 1121349 & Cataract Creek above Cataract Mine \\
\hline $35 \mathrm{M}$ & & $M: 128459$ & 462016 & 1121351 & Cataract Creek below Cataract Mine \\
\hline
\end{tabular}


Table 3. Historical water-quality sampling sites in the Boulder River watershed, Montana, with data in the Montana Bureau of Mines and Geology Ground Water Information Center database (Continued)

\begin{tabular}{|c|c|c|c|c|c|}
\hline $\begin{array}{c}\text { Site } \\
\text { number } \\
\text { (fig. 2) }\end{array}$ & $\begin{array}{l}\text { Analogous } \\
\text { USGS site } \\
\text { (fig. 2) }\end{array}$ & $\begin{array}{c}\text { MBMG site } \\
\text { number }\end{array}$ & Latitude & Longitude & Site name \\
\hline \multicolumn{6}{|c|}{ CATARACT CREEK--Continued } \\
\hline $36 \mathrm{M}$ & & $M: 128424$ & 462058 & 1121441 & Unnamed stream above Black Bear Mine \\
\hline $37 \mathrm{M}$ & & $M: 128427$ & -- & -- & Unnamed stream below Black Bear Mine \\
\hline $38 \mathrm{M}$ & & $M: 128452$ & 462002 & 1121443 & Upper Hattie Ferguson Mine tributary above mine \\
\hline $39 \mathrm{M}$ & 40 & M: 128448 & 461937 & 1121420 & Upper Hattie Ferguson Mine tributary at mouth \\
\hline $40 \mathrm{M}$ & & $M: 128191$ & 461916 & 1121427 & Unnamed tributary below Vera and Marie Mines \\
\hline $41 \mathrm{M}$ & 41 & $\begin{array}{l}M: 128543 \\
M: 122781\end{array}$ & $\begin{array}{l}461905 \\
461904\end{array}$ & $\begin{array}{l}1121442 \\
1121444\end{array}$ & Cataract Creek above Uncle Sam Gulch \\
\hline $42 \mathrm{M}$ & & $M: 122787$ & 462105 & 1121542 & Uncle Sam Gulch above Crystal Mine \\
\hline $43 \mathrm{M}$ & & $M: 121572$ & 462054 & 1121536 & Uncle Sam Gulch above Crystal Mine \\
\hline \multirow[t]{2}{*}{$44 \mathrm{M}$} & & $M: 121573$ & 462051 & 1121537 & Uncle Sam Gulch below Crystal Mine \\
\hline & & M:128541 & 462051 & 1121537 & \\
\hline $45 \mathrm{M}$ & & $M: 122785$ & 462042 & 1121540 & Uncle Sam Gulch 0.25 mi below Crystal Mine \\
\hline $46 \mathrm{M}$ & & $M: 122783$ & 462021 & 1121546 & Uncle Sam Gulch 0.5 mi below Crystal Mine \\
\hline $47 \mathrm{M}$ & 42 & $M: 122784$ & 462012 & 1121553 & Uncle Sam Gulch tributary below Crystal Mine \\
\hline $48 \mathrm{M}$ & & $M: 122782$ & 461935 & 1121540 & Uncle Sam Gulch 1.5 mi below Crystal Mine \\
\hline $49 \mathrm{M}$ & & $M: 122780$ & 461913 & 1121452 & Uncle Sam Gulch 2 mi below Crystal Mine \\
\hline $50 \mathrm{M}$ & 43 & $M: 128542$ & 461905 & 1121444 & Uncle Sam Gulch at mouth \\
\hline \multirow[t]{2}{*}{$51 \mathrm{M}$} & 44 & $M: 128544$ & 461902 & 1121444 & Cataract Creek below Uncle Sam Gulch \\
\hline & & $M: 121568$ & 461902 & 1121444 & \\
\hline $52 \mathrm{M}$ & & $M: 128545$ & 461845 & 1121500 & Cataract Creek $1,000 \mathrm{ft}$ below Phantom Mine \\
\hline $53 \mathrm{M}$ & & $M: 122773$ & 461826 & 1121510 & Cataract Creek $0.4 \mathrm{mi}$ above Deer Creek \\
\hline $54 \mathrm{M}$ & 45 & $M: 122772$ & 461807 & 1121518 & Deer Creek \\
\hline $55 \mathrm{M}$ & & $M: 137083$ & 461444 & 1121739 & Cataract $C$ reek $600 \mathrm{ft}$ above Mantle Mine \\
\hline $56 \mathrm{M}$ & & $M: 137082$ & -- & -. & Cataract Creek $1,600 \mathrm{ft}$ below Mantle Mine \\
\hline $57 \mathrm{M}$ & & $M: 137075$ & -- & -- & Big Limber Gulch above North Waldy Mine \\
\hline $58 \mathrm{M}$ & & M:137089 & -. & -. & Big Limber Gulch below North Waldy Mine above Waldy Mine \\
\hline $59 \mathrm{M}$ & & M:137093 & - & -- & Big Limber Gulch below Waldy Mine above Redwing Mine \\
\hline $60 \mathrm{M}$ & & $M: 137088$ & -- & -- & Big Limber Gulch $125 \mathrm{ft}$ below Redwing Mine \\
\hline $61 \mathrm{M}$ & & $M: 137097$ & -- & -- & Big Limber Gulch 0.25 mile below Redwing Mine \\
\hline $62 \mathrm{M}$ & & $M: 137090$ & -- & -- & Minneapolis Mine tributary at mouth \\
\hline $63 \mathrm{M}$ & & $M: 137076$ & -- & -- & Big Limber Gulch below Minneapolis Mine tributary \\
\hline $64 \mathrm{M}$ & 46 & $\begin{array}{l}M: 890813 \\
M: 137085\end{array}$ & $\begin{array}{c}461710 \\
--\end{array}$ & 1121425 & Big Limber Gulch at mouth \\
\hline $65 \mathrm{M}$ & & $M: 122776$ & 461651 & 1121449 & Cataract Creek below Big Limber Gulch \\
\hline $66 \mathrm{M}$ & & $M: 137084$ & -- & -- & Cataract Creek $1,600 \mathrm{ft}$ below South Mantle Mine \\
\hline $67 \mathrm{M}$ & 47 & $M: 121566$ & 461615 & 1121426 & Cataract Creek above Boulder River \\
\hline \multicolumn{6}{|c|}{ HIGH ORE CREEK } \\
\hline $72 \mathrm{M}$ & 49 & $M: 137333$ & -- & -- & High Ore Creek $0.9 \mathrm{mi}$ above Comet Mine \\
\hline $73 \mathrm{M}$ & & M:137322 & 461756 & 1121136 & High Ore Creek $0.3 \mathrm{mi}$ below Comet Mine \\
\hline $74 \mathrm{M}$ & & $M: 162780$ & 461818 & 1121106 & High Ore Creek above Bishop Creek \\
\hline $75 \mathrm{M}$ & & $M: 137324$ & 461822 & 1121101 & High Ore Creek below tributary at King Cole Mine \\
\hline $76 \mathrm{M}$ & 55 & $M: 137332$ & 461704 & 1121159 & Peters Gulch \\
\hline $77 \mathrm{M}$ & & $M: 137334$ & 461700 & 1121204 & High Ore Creek below Peters Gulch \\
\hline $78 \mathrm{M}$ & & M:137325 & 461648 & 1121219 & High Ore Creek above Golconda/Reliance Mine \\
\hline $79 \mathrm{M}$ & & $M: 137328$ & 461637 & 1121209 & High Ore Creek 0.3 mi below Golconda/Reliance Mine \\
\hline $80 \mathrm{M}$ & & $M: 137331$ & 461212 & 1121625 & High Ore Creek $0.6 \mathrm{mi}$ below Golconda/Reliance Mine \\
\hline $81 \mathrm{M}$ & 56 & $M: 137330$ & 461214 & 1121613 & High Ore Creek $0.85 \mathrm{mi}$ below Golconda/Reliance Mine \\
\hline
\end{tabular}


Table 4. Historical water-quality data retrieved from the Montana Bureau of Mines and Geology Ground Water Information Center database for streams in the Boulder River watershed, Montana

[Analyses by MBMG laboratory, Butte, Mont. Abbreviations: $\mathrm{ft}^{3} / \mathrm{s}$, cubic feet per second; $\mu \mathrm{g} / \mathrm{L}$, micrograms per liter; $\mu \mathrm{S} / \mathrm{cm}$, microsiemens per centimeter at 25 degrees Celsius; MBMG, Montana Bureau of Mines and Geology. Symbols: <, less than minimum reporting level; --, no data]

\begin{tabular}{|c|c|c|c|c|c|c|c|c|c|c|c|}
\hline $\begin{array}{c}\text { Site } \\
\text { number } \\
\text { (fig. 2) }\end{array}$ & Date & $\begin{array}{c}\text { Discharge, } \\
\text { instan- } \\
\text { taneous } \\
\left(\mathrm{ft}^{3} / \mathrm{s}\right)\end{array}$ & $\begin{array}{c}\text { Specific } \\
\text { conduc- } \\
\text { tance, } \\
\text { field } \\
(\mu S / \mathrm{cm})\end{array}$ & $\begin{array}{c}\text { pH, } \\
\text { field } \\
\text { (standard } \\
\text { units) }\end{array}$ & $\begin{array}{c}\text { Cadmium, } \\
\text { dis- } \\
\text { solved } \\
(\mu \mathrm{g} / \mathrm{L} \text { as } \mathrm{Cd})\end{array}$ & $\begin{array}{l}\text { Copper, } \\
\text { dissolved } \\
(\mu \mathrm{g} / \mathrm{L} \text { as } \\
\text { Cu })\end{array}$ & $\begin{array}{c}\text { Iron, } \\
\text { dissolved } \\
(\mu \mathrm{g} / \mathrm{L} \text { as } \\
\text { Fe })\end{array}$ & $\begin{array}{c}\text { Lead, } \\
\text { dissolved } \\
(\mu \mathrm{g} / \mathrm{L} \text { as } \\
\mathrm{Pb})\end{array}$ & $\begin{array}{c}\text { Zinc, } \\
\text { dissolved } \\
(\mu \mathrm{g} / \mathrm{L} \text { as } \\
\mathrm{Zn})\end{array}$ & $\begin{array}{l}\text { MBMG } \\
\text { laboratory } \\
\text { number }\end{array}$ & $\begin{array}{c}\text { MBMG } \\
\text { site } \\
\text { number }\end{array}$ \\
\hline $1 \mathrm{M}$ & $10-25-94$ & 12.6 & ${ }^{1} 163$ & 7.9 & $<2$ & $<2$ & 173 & $<2$ & 6.0 & $95 Q 0300$ & M:144336 \\
\hline $2 \mathrm{M}$ & $07-07-92$ & 1.0 & 58.2 & 6.7 & $<2$ & 3.7 & 71 & $<2$ & 10.7 & $92 \mathrm{Q} 0345$ & M:128487 \\
\hline $3 \mathrm{M}$ & $07-08-92$ & 3.4 & ${ }^{1} 68.8$ & 7.9 & $<5$ & 12.9 & 164 & 11.2 & 152.5 & $92 \mathrm{Q} 0355$ & $M: 128462$ \\
\hline $4 M$ & $09-25-89$ & 5.4 & 55 & 7.1 & 6 & 5 & 26 & $<50$ & 24 & $89 Q 1308$ & M: 890860 \\
\hline $5 \mathrm{M}$ & $07-15-92$ & 11.8 & 64.4 & 7.8 & $<2$ & 2.9 & 76 & $<2$ & 17.1 & $92 \mathrm{Q} 0461$ & M:128194 \\
\hline $6 \mathrm{M}$ & $06-26-92$ & .5 & 95.3 & 7.8 & $<2$ & 5.5 & 28 & $<2$ & 31.1 & $92 Q 0299$ & M: 128477 \\
\hline $7 \mathrm{M}$ & $06-26-92$ & .6 & 91.4 & 7.8 & $<2$ & 4.1 & 25 & $<3$ & 11.6 & $92 \mathrm{Q} 0300$ & M: 128478 \\
\hline $8 \mathrm{M}$ & $08-08-91$ & .1 & ${ }^{1} 114$ & 7.5 & 5 & $<5$ & 1,008 & $<100$ & 11 & 91Q0952 & M:123696 \\
\hline $9 \mathrm{M}$ & $08-08-91$ & .1 & -- & 7.7 & $<5$ & $<5$ & 64 & $<100$ & 26 & 91Q0955 & M: 123713 \\
\hline $10 \mathrm{M}$ & $08-08-91$ & .1 & - & 7.8 & 5 & $<5$ & $<3$ & $<100$ & $<5$ & $91 \mathrm{Q} 0953$ & $\mathrm{M}: 123712$ \\
\hline \multirow[t]{2}{*}{$11 \mathrm{M}$} & $07-06-92$ & 1.9 & 79.5 & 8.0 & $<2$ & 3.6 & 33 & $<2$ & 12.3 & $92 Q 0338$ & M:128490 \\
\hline & $07-13-92$ & 2.2 & 87.6 & 7.6 & $<2$ & 4.4 & 24 & $<2$ & 23.6 & $92 \mathrm{Q} 0418$ & M:128501 \\
\hline $12 \mathrm{M}$ & $06-30-92$ & 1.7 & 66.6 & 7.1 & $<2$ & 4.9 & 60 & $<2$ & 31.7 & $92 Q 0309$ & M: 128468 \\
\hline \multirow[t]{2}{*}{$13 \mathrm{M}$} & $07-01-92$ & 1.8 & 181 & 6.6 & 21.8 & 80.2 & 231 & $<2$ & 2,750 & $92 \mathrm{Q} 0316$ & M: 128482 \\
\hline & $07-13-92$ & 1.1 & 149 & 6.3 & 52.2 & 116.8 & 751 & $<2$ & 3,220 & $92 \mathrm{Q} 0413$ & M:128494 \\
\hline \multirow[t]{2}{*}{$14 \mathrm{M}$} & $07-06-92$ & 1.1 & 128 & 7.0 & 18.9 & 38.2 & 46 & $<2$ & 2,230 & $92 \mathrm{Q} 0339$ & M:128491 \\
\hline & $07-13-92$ & -- & 134 & 7.0 & 19.1 & 35.5 & 20 & $<2$ & 2,290 & $92 \mathrm{Q} 0421$ & M:128504 \\
\hline $15 \mathrm{M}$ & $07-06-92$ & 5.2 & 82.5 & 7.5 & 4.0 & 21.7 & 59 & $<2$ & 373.2 & $92 Q 0337$ & M:128489 \\
\hline $16 \mathrm{M}$ & $08-08-91$ & 1.5 & ${ }^{1} 120$ & 7.2 & 12 & 38 & 192 & 105 & 694 & $91 \mathrm{Q} 0939$ & M:123716 \\
\hline $17 \mathrm{M}$ & $08-08-91$ & .4 & - & 7.5 & $<5$ & $<5$ & -- & $<100$ & 7 & $91 \mathrm{Q} 0956$ & M:123715 \\
\hline \multirow[t]{3}{*}{$18 \mathrm{M}$} & $08-08-91$ & 1.2 & - & 7.6 & $<2$ & $<5$ & 6 & $<100$ & 14 & $91 \mathrm{Q} 0947$ & M:123702 \\
\hline & $07-01-92$ & 1.5 & 66.2 & 6.9 & $<2$ & 2.5 & 33 & $<2$ & 17.0 & $92 Q 0315$ & M:128471 \\
\hline & $07-13-92$ & 1.0 & 71.4 & 7.6 & $<2$ & $<2$ & 9 & $<2$ & 6.0 & $92 Q 0415$ & M:128496 \\
\hline \multirow[t]{2}{*}{$19 \mathrm{M}$} & $07-06-92$ & 1.6 & 75.4 & 7.7 & $<5$ & $<2$ & 31 & $<2$ & 5.5 & $92 \mathrm{Q} 0340$ & M:128492 \\
\hline & $07-13-92$ & 1.5 & 71.5 & 7.6 & $<2$ & 2.3 & 16 & $<2$ & 8.7 & $92 \mathrm{Q} 0416$ & M:128497 \\
\hline $20 \mathrm{M}$ & $08-08-91$ & 1.8 & - & 7.5 & $<5$ & 11 & 135 & $<100$ & 324 & $91 \mathrm{Q} 0957$ & $M: 123717$ \\
\hline $21 \mathrm{M}$ & $07-05-92$ & 5.3 & 87.6 & 7.8 & 3.7 & 16.4 & 67 & $<2$ & 206 & $92 \mathrm{Q} 0463$ & M:128196 \\
\hline $22 \mathrm{M}$ & $09-25-89$ & - & 81.4 & 7.2 & -- & 17 & 86 & $<50$ & 178 & $89 \mathrm{Q} 1309$ & $M: 890861$ \\
\hline $23 \mathrm{M}$ & $07-15-92$ & 18.9 & 74.6 & 7.4 & $<2$ & $<2$ & 73 & $<2$ & 98.4 & $92 \mathrm{Q} 0462$ & M:128195 \\
\hline $24 \mathrm{M}$ & $04-14-92$ & -- & 309 & 8.2 & $<2$ & $<2$ & 7 & $<2$ & 29 & $92 \mathrm{Q} 0437$ & $M: 128511$ \\
\hline $25 \mathrm{M}$ & $07-15-92$ & 23.0 & 84.6 & 7.8 & $<2$ & 7.8 & 222 & $<2$ & 54.7 & $92 \mathrm{Q} 0464$ & $M: 128197$ \\
\hline \multirow[t]{2}{*}{$26 \mathrm{M}$} & $07-25-91$ & - & ${ }^{1} 82.8$ & 7.8 & $<6$ & $<6$ & 150 & - & 25 & $91 \mathrm{Q} 0323$ & $\mathrm{M}: 122779$ \\
\hline & $10-22-91$ & -- & ${ }^{1} 147$ & 8.3 & $<6$ & 17 & 124 & - & 70 & 91Q1116 & M:122779 \\
\hline $27 \mathrm{M}$ & $08-05-92$ & .042 & 63.2 & 7.3 & $<2$ & $<2$ & 28 & $<2$ & 5.6 & $92 \mathrm{Q} 0721$ & $M: 134779$ \\
\hline $28 \mathrm{M}$ & $08-05-92$ & .056 & 65.8 & 7.5 & $<2$ & $<2$ & 24 & $<2$ & 27.1 & $92 Q 0720$ & M:134782 \\
\hline $29 \mathrm{M}$ & $08-04-92$ & .037 & 58.1 & 7.2 & $<2$ & 2.2 & 71 & $<2$ & 4.2 & $92 \mathrm{Q} 0684$ & $M: 132718$ \\
\hline $30 \mathrm{M}$ & $08-04-92$ & .045 & 81.9 & 6.8 & $<2$ & 2.7 & 180 & $<2$ & 4.8 & $92 Q 0687$ & M:132720 \\
\hline
\end{tabular}


Table 4. Historical water-quality data retrieved from the Montana Bureau of Mines and Geology Ground Water Information Center database for streams in the Boulder River Abandoned Mine Lands lnitiative study area, Montana (Continued)

\begin{tabular}{|c|c|c|c|c|c|c|c|c|c|c|c|}
\hline $\begin{array}{c}\text { Site } \\
\text { number } \\
\text { (fig. 2) }\end{array}$ & Date & $\begin{array}{c}\text { Discharge, } \\
\text { instan- } \\
\text { taneous } \\
\left(\mathrm{ft}^{3} / \mathbf{s}\right)\end{array}$ & $\begin{array}{c}\text { Specific } \\
\text { conduc- } \\
\text { tance, } \\
\text { field } \\
(\mu \mathrm{S} / \mathrm{cm})\end{array}$ & $\begin{array}{c}\text { pH, } \\
\text { field } \\
\text { (standard } \\
\text { units) }\end{array}$ & $\begin{array}{c}\text { Cadmium, } \\
\text { dis- } \\
\text { solved } \\
(\mu \mathrm{g} / \mathrm{L} \text { as } \mathrm{Cd})\end{array}$ & $\begin{array}{l}\text { Copper, } \\
\text { dissolved } \\
(\mu \mathrm{g} / \mathrm{L} \text { as } \\
\mathrm{Cu})\end{array}$ & $\begin{array}{l}\text { Iron, } \\
\text { dissolved } \\
(\mu \mathrm{g} / \mathrm{L} \text { as } \\
\text { Fe })\end{array}$ & $\begin{array}{c}\text { Lead, } \\
\text { dissolved } \\
(\mu \mathrm{g} / \mathrm{L} \text { as } \\
\mathrm{Pb})\end{array}$ & $\begin{array}{c}\text { Zinc, } \\
\text { dissolved } \\
(\mu \mathrm{g} / \mathrm{L} \text { as } \\
\mathrm{Zn})\end{array}$ & $\begin{array}{l}\text { MBMG } \\
\text { laboratory } \\
\text { number }\end{array}$ & $\begin{array}{c}\text { MBMG } \\
\text { site } \\
\text { number }\end{array}$ \\
\hline $31 \mathrm{M}$ & 08-04-92 & .045 & 97.4 & 5.8 & $<2$ & 159 & 65 & $<2$ & 190 & $92 \mathrm{Q} 0686$ & M:132719 \\
\hline $32 \mathrm{M}$ & $07-29-92$ & .018 & 81.1 & 7.1 & $<2$ & 2.8 & 65 & $<2$ & 41 & $92 Q 0659$ & M:128549 \\
\hline \multirow[t]{2}{*}{$33 \mathrm{M}$} & $07-27-92$ & 4.4 & 72.3 & 7.5 & $<2$ & 3.0 & 200 & $<2$ & 14.2 & $92 \mathrm{Q} 0626$ & M:128539 \\
\hline & $07-27-92$ & 4.4 & 77.2 & 7.8 & $<2$ & 3.1 & 195 & $<2$ & 13.3 & $92 \mathrm{Q} 0625$ & M:128538 \\
\hline $34 \mathrm{M}$ & $07-22-92$ & 11.5 & 66.2 & 7.6 & 9.5 & 7.6 & 120 & $<2$ & 34.6 & $92 \mathrm{Q} 0483$ & M:128456 \\
\hline $35 \mathrm{M}$ & $07-22-92$ & 10.7 & 70.1 & 7.7 & $<2$ & 7.8 & 130 & $<2$ & 38.6 & $92 \mathrm{Q} 0487$ & M: 128459 \\
\hline $36 \mathrm{M}$ & $07-30-92$ & .04 & -- & 7.0 & $<2$ & $<2$ & 21 & $<2$ & 20.2 & $92 \mathrm{Q} 0668$ & M: 128424 \\
\hline $37 \mathrm{M}$ & $07-30-92$ & .04 & 64.2 & 6.8 & 7.6 & 61.3 & 26 & $<2$ & 714 & $92 \mathrm{Q} 0671$ & M128427 \\
\hline $38 \mathrm{M}$ & $07-23-92$ & .017 & 320 & 7.7 & 3.6 & $<2$ & 20 & $<2$ & 1,350 & $92 \mathrm{Q} 0505$ & M:128452 \\
\hline $39 \mathrm{M}$ & $07-23-92$ & .11 & 95.3 & 7.2 & $<2$ & 8.0 & 141 & $<2$ & 400 & $92 \mathrm{Q} 0501$ & M:128448 \\
\hline $40 \mathrm{M}$ & $07-20-92$ & .1 & 150 & 7.7 & $<2$ & $<2$ & 34 & $<2$ & 7.5 & $92 Q 0479$ & M128191 \\
\hline \multirow[t]{3}{*}{$41 M$} & $06-25-91$ & 38.6 & ${ }^{1} 60.6$ & 7.7 & $<6$ & 14 & 154 & - & 34 & 91Q0322 & M:122781 \\
\hline & $10-22-91$ & -- & ${ }^{1} 124$ & 8.0 & $<6$ & $<6$ & 91 & -- & 77 & $91 Q 1114$ & M:122781 \\
\hline & $07-28-92$ & 6.0 & 98.6 & 8.0 & $<2$ & 6.0 & 116 & $<2$ & 42.6 & $92 Q 0641$ & M:128543 \\
\hline $42 \mathrm{M}$ & $06-27-91$ & .5 & ${ }^{1} 27.3$ & 6.5 & $<6$ & 9 & 78 & -- & $<6$ & 91Q0311 & M:122787 \\
\hline $43 M$ & $-\quad-91$ & - & ${ }^{1} 62.8$ & 7.2 & 6 & 24 & 175 & $\cdots$ & 134 & $90 \mathrm{Q} 0439$ & M:121572 \\
\hline $44 \mathrm{M}$ & $07-28-92$ & .25 & 434 & 3.5 & 215.5 & 4,850 & 4,540 & 78.6 & 21,000 & $92 \mathrm{Q} 0639$ & M:128541 \\
\hline $45 \mathrm{M}$ & $06-26-91$ & 1.3 & ${ }^{1} 273$ & 3.7 & 115 & 2,850 & 3,730 & -- & 9,550 & $91 \mathrm{Q} 0312$ & M:122785 \\
\hline $46 \mathrm{M}$ & $06-26-91$ & - & ${ }^{1} 149$ & 4.7 & 60 & 1,520 & 1,160 & -- & 5,250 & $91 \mathrm{Q} 0328$ & $\mathrm{M}: 122783$ \\
\hline $47 \mathrm{M}$ & $06-26-91$ & - & ${ }^{1} 98.0$ & 6.6 & $<6$ & $<6$ & 28 & -- & 54 & $91 \mathrm{Q} 0327$ & M:122784 \\
\hline $48 \mathrm{M}$ & $06-26-91$ & -- & -- & 6.4 & 22 & 235 & 126 & -- & 1,630 & $91 Q 0313$ & $M: 122782$ \\
\hline \multirow[t]{2}{*}{$49 \mathrm{M}$} & $06-26-91$ & 5.1 & ${ }^{1} 78.6$ & 6.9 & 12 & 183 & 84 & -- & 1,450 & $91 \mathrm{Q} 0314$ & $\mathrm{M}: 122780$ \\
\hline & $10-22-91$ & -- & ${ }^{1} 135$ & 6.5 & 59 & 312 & 12 & -- & 5,160 & $91 \mathrm{Q} 1112$ & $\mathrm{M}: 122780$ \\
\hline $50 \mathrm{M}$ & $07-28-92$ & 1.1 & 155 & 7.1 & 44.6 & 76 & 8 & $<2$ & 3,150 & $92 \mathrm{Q} 0640$ & M:128542 \\
\hline \multirow[t]{3}{*}{$51 \mathrm{M}$} & $06-25-91$ & 43.2 & ${ }^{1} 62.8$ & 7.4 & $<6$ & 43 & 141 & -- & 185 & $91 Q 0315$ & M:121568 \\
\hline & $10-22-91$ & -- & ${ }^{1} 149$ & 7.8 & 8 & 61 & 71 & -- & 802 & 91Q1110 & M:121568 \\
\hline & $07-28-92$ & 7.1 & 119 & 7.9 & 3.0 & 27.2 & 87 & $<2$ & 372.9 & $92 \mathrm{Q} 0642$ & $\mathrm{M}: 128544$ \\
\hline $52 \mathrm{M}$ & $07-28-92$ & 5.7 & 107 & 7.7 & 4.7 & 35.2 & 71 & $<2$ & 477.4 & $92 \mathrm{Q} 0643$ & M:128545 \\
\hline $53 \mathrm{M}$ & $06-25-91$ & 43.2 & ${ }^{1} 64.1$ & 7.6 & $<6$ & 25 & 121 & -- & 178 & $91 \mathrm{Q} 0316$ & M:122773 \\
\hline $54 \mathrm{M}$ & $06-25-91$ & .5 & ${ }^{1} 120$ & 7.9 & $<6$ & $<6$ & 80 & -- & 40 & 91Q0325 & M:122772 \\
\hline $55 \mathrm{M}$ & $10-13-93$ & 13.7 & 97.2 & 7.9 & 3.2 & 44.9 & 121 & $<2$ & 278 & $94 Q 0767$ & M:137083 \\
\hline $56 \mathrm{M}$ & $10-13-93$ & 14.4 & 90.6 & 8.2 & 3.4 & 39.5 & 124 & $<2$ & 277 & $94 Q 0765$ & M:137082 \\
\hline $57 \mathrm{M}$ & $10-12-93$ & .017 & 81.9 & 6.6 & $<2$ & $<2$ & 24 & $<2$ & 3.5 & $94 Q 0751$ & M:137075 \\
\hline $58 \mathrm{M}$ & $10-12-93$ & .015 & 149 & 7.3 & $<2$ & 3.6 & 28 & $<2$ & 9.1 & $94 Q 0779$ & M:137089 \\
\hline $59 \mathrm{M}$ & $10-12-93$ & .049 & 233 & 7.6 & $<2$ & $<2$ & 40 & $<2$ & 4.8 & $94 Q 0743$ & M:137093 \\
\hline $60 \mathrm{M}$ & $10-12-93$ & .049 & 207 & 7.6 & $<2$ & 3.2 & 33 & $<2$ & 38.8 & 94Q0777 & M:137088 \\
\hline $61 \mathrm{M}$ & $10-12-93$ & .045 & 206 & 7.3 & $<2$ & 8.7 & 61 & $<2$ & 33.5 & $94 Q 0747$ & M:137097 \\
\hline $62 \mathrm{M}$ & $10-14-93$ & .030 & 384 & 7.6 & $<2$ & 2.6 & 32 & 2.5 & 6.7 & $94 Q 0781$ & M:137090 \\
\hline $63 \mathrm{M}$ & $10-14-93$ & .11 & 305 & 7.7 & $<2$ & $<2$ & 44 & $<2$ & 4.9 & $94 Q 0753$ & M:137076 \\
\hline
\end{tabular}


Table 4. Historical water-quality data retrieved from the Montana Bureau of Mines and Geology Ground Water Information Center database for streams in the Boulder River Abandoned Mine Lands Initiative study area, Montana (Continued)

\begin{tabular}{|c|c|c|c|c|c|c|c|c|c|c|c|}
\hline $\begin{array}{c}\text { Site } \\
\text { number } \\
\text { (fig. 2) }\end{array}$ & Date & $\begin{array}{c}\text { Discharge, } \\
\text { instan- } \\
\text { taneous } \\
\left(\mathrm{ft}^{3} / \mathrm{s}\right)\end{array}$ & $\begin{array}{c}\text { Specific } \\
\text { conduc- } \\
\text { tance, } \\
\text { field } \\
(\mu \mathrm{S} / \mathrm{cm})\end{array}$ & $\begin{array}{c}\text { pH, } \\
\text { field } \\
\text { (standard } \\
\text { units) }\end{array}$ & $\begin{array}{c}\text { Cadmium, } \\
\text { dis- } \\
\text { solved } \\
(\mu \mathrm{g} / \mathrm{L} \text { as } \mathrm{Cd})\end{array}$ & $\begin{array}{l}\text { Copper, } \\
\text { dissolved } \\
(\mu \mathrm{g} / \mathrm{L} \text { as } \\
\mathrm{Cu})\end{array}$ & $\begin{array}{c}\text { Iron, } \\
\text { dissolved } \\
(\mu \mathrm{g} / \mathrm{L} \text { as } \\
\text { Fe })\end{array}$ & $\begin{array}{c}\text { Lead, } \\
\text { dissolved } \\
(\mu \mathrm{g} / \mathrm{L} \text { as } \\
\text { Pb })\end{array}$ & $\begin{array}{c}\text { Zinc, } \\
\text { dissolved } \\
(\mu \mathrm{g} / \mathrm{L} \text { as } \\
\mathrm{Zn})\end{array}$ & $\begin{array}{c}\text { MBMG } \\
\text { laboratory } \\
\text { number }\end{array}$ & $\begin{array}{c}\text { MBMG } \\
\text { site } \\
\text { number }\end{array}$ \\
\hline \multirow[t]{2}{*}{$64 M$} & $06-25-91$ & .2 & $1_{248}$ & 8.1 & $<6$ & $<6$ & 14 & -- & $<6$ & $91 Q 0324$ & $\mathrm{M}: 890813$ \\
\hline & $10-13-93$ & .1 & 300 & 8.0 & $<2$ & $<2$ & 26 & $<2$ & 4.9 & $94 \mathrm{Q} 0771$ & $\mathrm{M}: 137085$ \\
\hline \multirow[t]{2}{*}{$65 \mathrm{M}$} & $06-25-91$ & 43.8 & ${ }^{1} 67.3$ & 7.6 & $<6$ & 29 & 111 & -- & 165 & $91 Q 0317$ & M:122776 \\
\hline & $11-16-91$ & -- & ${ }^{1} 138$ & 7.8 & $<6$ & 47 & 41 & - & 528 & 91Q1118 & M: 122776 \\
\hline $66 \mathrm{M}$ & $10-13-93$ & 11.6 & $1_{99.2}$ & 7.8 & 3.1 & 40.7 & 109 & $<2$ & 252 & $94 Q 0769$ & M:137084 \\
\hline $67 \mathrm{M}$ & $-\quad-91$ & -- & ${ }^{1} 178$ & 7.8 & 6 & 27 & 167 & -- & 634 & $90 \mathrm{Q} 0432$ & M:121566 \\
\hline $68 \mathrm{M}$ & $-\quad-91$ & -- & ${ }^{1} 142$ & 8.2 & $<5$ & 13 & 78 & -- & 159 & $90 \mathrm{Q} 0434$ & $M: 121565$ \\
\hline \multirow[t]{2}{*}{$69 \mathrm{M}$} & $06-25-91$ & -- & ${ }^{1} 80.5$ & 7.6 & $<6$ & 17 & 154 & -- & 51 & $91 Q 0318$ & $\mathrm{M}: 122777$ \\
\hline & $10-22-91$ & -- & ${ }^{1} 160$ & 8.1 & $<6$ & 27 & 84 & -- & 134 & 91Q1108 & M:122777 \\
\hline $70 \mathrm{M}$ & $06-25-91$ & -- & $1_{83.0}$ & 7.6 & $<6$ & 8 & 148 & -- & 49 & $91 \mathrm{Q} 0319$ & M: 122778 \\
\hline $71 \mathrm{M}$ & $06-25-91$ & - & $1_{85.5}$ & 7.9 & $<6$ & 17 & 148 & -- & 48 & $91 \mathrm{Q} 0320$ & M:122775 \\
\hline $72 \mathrm{M}$ & $09-28-93$ & .4 & 105 & 7.8 & $<2$ & 2.0 & 100 & $<2$ & 54 & $94 Q 0707$ & M:137333 \\
\hline $73 \mathrm{M}$ & $09-29-93$ & .7 & 454 & 7.1 & 6.5 & 3.8 & 198 & $<2$ & 4,145 & $94 Q 0687$ & $\mathrm{M}: 137322$ \\
\hline \multirow[t]{2}{*}{$74 \mathrm{M}$} & $08-26-97$ & 1.4 & 458 & 8.1 & 9.4 & 2.5 & 14 & $<2$ & 4,423 & $98 \mathrm{Q} 0224$ & M: 162780 \\
\hline & $08-27-97$ & 1.7 & 431 & 8.3 & 9.0 & 2.7 & 32 & $<2$ & 5,214 & $98 \mathrm{Q} 0230$ & M: 162780 \\
\hline $75 \mathrm{M}$ & $09-29-93$ & 1.2 & 443 & 6.5 & 6.7 & 5.2 & 177 & $<2$ & 3,736 & $94 Q 0691$ & M: 137324 \\
\hline $76 \mathrm{M}$ & $09-28-93$ & .006 & 381 & 8.9 & $<2$ & $<2$ & 6 & $<2$ & 63.9 & $94 \mathrm{Q} 0705$ & M:137332 \\
\hline $77 \mathrm{M}$ & $09-28-93$ & 1.1 & 380 & 8.6 & 3.9 & 5.9 & 103 & 2.0 & 1,201 & $94 \mathrm{Q} 0709$ & M:137334 \\
\hline $78 \mathrm{M}$ & $09-28-93$ & 1.5 & 388 & 8.1 & 5.0 & 5.3 & 108 & $<2$ & 1,659 & $94 \mathrm{Q} 0693$ & M:137325 \\
\hline $79 \mathrm{M}$ & $09-28-93$ & 1.4 & 396 & 8.0 & 4.8 & 4.4 & 97 & $<2$ & 1,776 & $94 Q 0697$ & $M: 137328$ \\
\hline $80 \mathrm{M}$ & $09-28-93$ & 1.6 & 410 & 7.7 & 5.3 & 4.9 & 95 & 3.6 & 2,000 & $94 Q 0703$ & $\mathrm{M}: 137331$ \\
\hline $81 \mathrm{M}$ & $09-28-93$ & 1.3 & 402 & 6.9 & 5.6 & 5.1 & 107 & $<2$ & 1,970 & $94 \mathrm{Q} 0701$ & M: 137330 \\
\hline $82 \mathrm{M}$ & $10-13-93$ & .045 & 382 & 6.9 & $<2$ & 4.7 & 10 & $<2$ & 19 & $94 \mathrm{Q} 0761$ & M:137080 \\
\hline $83 \mathrm{M}$ & $10-13-93$ & .1 & 392 & 6.5 & $<2$ & $<2$ & 16 & $<2$ & 7.6 & $94 Q 0773$ & M:137086 \\
\hline
\end{tabular}

${ }^{1}$ Specific-conductance value measured in laboratory. 
Table 5. Water-quality data for field duplicates, Boulder River watershed, Montana

[Samples collected by the U.S. Geological Survey. ANC, acid-neutralizing capacity determined on unfiltered samples, formerly referred to as alkalinity. Abbreviations: lab, laboratory; $\mu \mathrm{g} / \mathrm{L}$, micrograms per liter; $\mu \mathrm{S} / \mathrm{cm}$, microsiemens per centimeter at 25 degrees Celsius; mg/L, milligrams per liter. Symbols: $<$, less than minimum reporting level; --, no data]

\begin{tabular}{|c|c|c|c|c|c|c|c|c|c|c|}
\hline $\begin{array}{c}\text { Site } \\
\text { number } \\
\text { (fig. 2) }\end{array}$ & Date & Time & $\begin{array}{c}\text { Specific } \\
\text { conduc- } \\
\text { tance, } \\
\text { lab } \\
(\mu \mathrm{S} / \mathrm{cm})\end{array}$ & $\begin{array}{c}\text { pH, } \\
\text { lab } \\
\text { (standard } \\
\text { units) }\end{array}$ & $\begin{array}{c}\text { Hard- } \\
\text { ness, } \\
\text { total } \\
(\mathrm{mg} / \mathrm{L} \text { as } \\
\left.\mathrm{CaCO}_{3}\right)\end{array}$ & $\begin{array}{l}\text { Calcium, } \\
\text { dissolved } \\
(\mathrm{mg} / \mathrm{L} \text { as } \\
\text { Ca) }\end{array}$ & $\begin{array}{l}\text { Magne- } \\
\text { sium, } \\
\text { dissolved } \\
\text { (mg/L as } \\
\quad \text { Mg) }\end{array}$ & $\begin{array}{c}\text { Sodium, } \\
\text { dissolved } \\
\text { (mg/L as } \\
\text { Na) }\end{array}$ & $\begin{array}{c}\text { Potas- } \\
\text { sium, } \\
\text { dissolved } \\
(\mathrm{mg} / \mathrm{L} \text { as } \mathrm{K})\end{array}$ & $\begin{array}{c}\mathrm{ANC}, \\
\mathrm{lab} \\
(\mathrm{mg} / \mathrm{l} \text { as } \\
\left.\mathrm{CaCO}_{3}\right)\end{array}$ \\
\hline \multirow[t]{2}{*}{24} & $12-03-96$ & 1245 & 107 & 7.3 & 39 & 11 & 2.7 & 3.6 & 1.3 & 29 \\
\hline & $12-03-96$ & 1250 & 108 & 6.9 & 41 & 12 & 2.7 & 3.8 & 1.2 & 30 \\
\hline \multirow[t]{2}{*}{56} & $05-09-97$ & 1500 & 238 & 7.7 & 96 & 27 & 6.9 & 4.5 & 1.4 & 60 \\
\hline & $05-09-97$ & 1501 & 237 & 7.7 & 99 & 28 & 7.1 & 4.6 & 1.4 & 60 \\
\hline \multirow[t]{2}{*}{58} & $11-01-96$ & 1200 & 177 & 7.2 & 68 & 20 & 4.3 & 7.6 & 1.5 & 60 \\
\hline & $11-01-96$ & 1205 & 178 & 7.6 & 65 & 19 & 4.3 & 7.8 & 1.6 & 60 \\
\hline \multirow[t]{2}{*}{59} & $05-24-97$ & 0930 & 58 & 7.5 & 21 & 6.2 & 1.3 & 2.8 & .96 & 19 \\
\hline & $05-24-97$ & 0935 & 58 & 7.3 & 21 & 6.2 & 1.4 & 2.8 & .96 & 19 \\
\hline \multirow[t]{2}{*}{25} & $02-11-98$ & 1430 & 151 & 7.8 & 55 & 16 & 3.6 & 7.4 & 1.2 & 53 \\
\hline & $02-11-98$ & 1431 & 151 & 7.7 & 55 & 16 & 3.5 & 7.4 & 1.2 & 51 \\
\hline \multirow[t]{2}{*}{44} & $05-06-98$ & 1415 & 51 & 7.2 & 20 & 6.2 & 1.1 & 1.6 & .83 & 17 \\
\hline & $05-06-98$ & 1420 & 51 & 6.9 & 20 & 6.2 & 1.1 & 1.6 & .85 & 17 \\
\hline \multirow[t]{2}{*}{17} & $10-31-96$ & 1600 & 247 & 5.8 & 86 & 24 & 6.4 & 3.2 & 1.1 & 1.3 \\
\hline & $10-31-96$ & 1605 & 249 & 4.9 & 83 & 23 & 6.3 & 3.3 & 1.1 & 1.4 \\
\hline \multirow[t]{2}{*}{8} & $09-13-99$ & 1250 & 82 & 7.6 & 33 & 9.5 & 2.3 & 2.3 & 1.1 & 32 \\
\hline & $09-13-99$ & 1251 & 82 & 7.6 & 34 & 9.6 & 2.4 & 2.3 & 1.1 & 34 \\
\hline
\end{tabular}

\begin{tabular}{|c|c|c|c|c|c|c|c|c|c|c|c|}
\hline $\begin{array}{c}\text { Site } \\
\text { number } \\
\text { (fig. 2) }\end{array}$ & Date & $\begin{array}{l}\text { Sulfate, } \\
\text { dissolved } \\
(\mathrm{mg} / \mathrm{L} \text { as } \\
\left.\mathrm{SO}_{4}\right)\end{array}$ & $\begin{array}{c}\text { Chloride, } \\
\text { dissolved } \\
\text { (mg/L as } \\
\text { Cl) }\end{array}$ & $\begin{array}{l}\text { Fluoride, } \\
\text { dissolved } \\
(\mathrm{mg} / \mathrm{L} \text { as } \mathrm{F})\end{array}$ & $\begin{array}{c}\text { Silica, } \\
\text { dissolved } \\
(\mathrm{mg} / \mathrm{L} \text { as } \\
\left.\mathrm{SiO}_{2}\right)\end{array}$ & $\begin{array}{l}\text { Nitrite, } \\
\text { dissolved } \\
\text { (mg/L as } \\
\text { N) }\end{array}$ & $\begin{array}{l}\text { Nitrite plus } \\
\text { nitrate, } \\
\text { dissolved } \\
(\mathrm{mg} / \mathrm{L} \text { as } \\
\mathrm{N})\end{array}$ & $\begin{array}{l}\text { Ammonia, } \\
\text { dissolved } \\
(\mathrm{mg} / \mathrm{L} \text { as } \mathbf{N})\end{array}$ & $\begin{array}{c}\text { Phos- } \\
\text { phorus, } \\
\text { ortho, } \\
\text { dissolved } \\
\text { (mg/L as P) }\end{array}$ & $\begin{array}{l}\text { Alum- } \\
\text { inum, } \\
\text { total } \\
\text { recov- } \\
\text { erable } \\
(\mu \mathrm{g} / \mathrm{L} \text { as } \\
\text { Al) }\end{array}$ & $\begin{array}{l}\text { Alum- } \\
\text { inum, } \\
\text { dissolved } \\
(\mu \mathrm{g} / \mathrm{L} \text { as } \\
\text { Al) }\end{array}$ \\
\hline \multirow[t]{2}{*}{24} & $12-03-96$ & 20 & 1.0 & 0.10 & 17 & $<0.01$ & 0.06 & 0.030 & $<0.010$ & -- & 6.5 \\
\hline & $12-03-96$ & 19 & 2.3 & $<.10$ & 17 & .01 & .05 & .030 & $<.010$ & -- & 6.4 \\
\hline \multirow[t]{2}{*}{56} & $05-09-97$ & 50 & 1.4 & .12 & 17 & $<.01$ & .12 & $<.015$ & $<.010$ & -- & 63 \\
\hline & $05-09-97$ & 50 & 1.5 & .14 & 17 & $<.01$ & .14 & $<.015$ & $<.010$ & - & 67 \\
\hline \multirow[t]{2}{*}{58} & $11-01-96$ & 24 & 1.9 & .10 & 21 & -- & -- & -- & -- & -- & 12 \\
\hline & $11-01-96$ & 24 & 1.9 & .10 & 21 & -- & -- & -- & -- & -- & 11 \\
\hline \multirow[t]{2}{*}{59} & $05-24-97$ & 6.7 & .61 & $<.10$ & 14 & -- & -- & -- & - & 2,050 & 68 \\
\hline & $05-24-97$ & 6.7 & .59 & $<.10$ & 14 & -- & -- & -- & - & - & 72 \\
\hline \multirow[t]{2}{*}{25} & $02-11-98$ & 16 & 2.1 & $<.10$ & 21 & $<.01$ & .13 & .030 & .023 & 40 & 5.6 \\
\hline & $02-11-98$ & 16 & 2.2 & $<.10$ & 21 & $<.01$ & .14 & .026 & .027 & 52 & 5.2 \\
\hline \multirow[t]{2}{*}{44} & $05-06-98$ & 5.6 & .34 & $<.10$ & 9.5 & -- & -- & -- & -- & 330 & 57 \\
\hline & $05-06-98$ & 5.7 & .36 & $<.10$ & 9.7 & -- & -- & -- & -- & 300 & 52 \\
\hline \multirow[t]{2}{*}{17} & $10-31-96$ & 100 & .40 & .10 & 21 & -- & - & -- & -- & -- & 249 \\
\hline & $10-31-96$ & 100 & .40 & .10 & 21 & - & -- & -- & -- & -- & 254 \\
\hline \multirow[t]{2}{*}{8} & $09-13-99$ & 8.0 & $<.29$ & -- & -- & -. & -. & -- & -- & 39 & 26 \\
\hline & $09-13-99$ & 8.0 & $<.29$ & -- & -- & -- & -- & -- & - & 42 & 25 \\
\hline
\end{tabular}


Table 5. Water-quality data for field duplicates, Boulder River watershed, Montana (Continued)

\begin{tabular}{|c|c|c|c|c|c|c|c|c|c|c|c|}
\hline $\begin{array}{c}\text { Site } \\
\text { number } \\
\text { (fig. 2) }\end{array}$ & Date & $\begin{array}{c}\text { Antimony, } \\
\text { dissolved } \\
(\mu \mathrm{g} / \mathrm{L} \text { as } \\
\text { Sb) }\end{array}$ & $\begin{array}{c}\text { Arsenic, } \\
\text { total } \\
\text { recov- } \\
\text { erable } \\
(\mu \mathrm{g} / \mathrm{L} \text { as } \\
\text { As) }\end{array}$ & $\begin{array}{c}\text { Arsenic, } \\
\text { dissolved } \\
(\mu \mathrm{g} / \mathrm{L} \text { as } \\
\text { As })\end{array}$ & $\begin{array}{c}\text { Barium, } \\
\text { dissolved } \\
(\mu \mathrm{g} / \mathrm{L} \text { as Ba })\end{array}$ & $\begin{array}{l}\text { Beryllium, } \\
\text { dissolved, } \\
(\mu \mathrm{g} / \mathrm{L} \text { as } \\
\text { Be })\end{array}$ & $\begin{array}{c}\text { Cadmium, } \\
\text { total } \\
\text { recov- } \\
\text { erable } \\
(\mu \mathrm{g} / \mathrm{L} \text { as } \\
\text { Cd) }\end{array}$ & $\begin{array}{c}\text { Cadmium, } \\
\text { dissolved } \\
\text { ( } \mu \mathrm{g} / \mathrm{L} \text { as } \\
\text { Cd) }\end{array}$ & $\begin{array}{c}\text { Chromium, } \\
\text { total } \\
\text { recov- } \\
\text { erable } \\
(\mu \mathrm{g} / \mathrm{L} \text { as } \\
\text { Cr) }\end{array}$ & $\begin{array}{c}\text { Chromium, } \\
\text { dissolved } \\
(\mu \mathrm{g} / \mathrm{L} \text { as } \mathrm{Cr})\end{array}$ & $\begin{array}{c}\text { Cobalt, } \\
\text { dissolved } \\
(\mu \mathrm{g} / \mathrm{L} \text { as } \\
\text { Co) }\end{array}$ \\
\hline \multirow[t]{2}{*}{24} & $12-03-96$ & $<1$ & 5 & 4 & 23 & $<1$ & $<1$ & 0.4 & $<1$ & $<1$ & $<1$ \\
\hline & $12-03-96$ & $<1$ & 5 & 4 & 24 & $<1$ & $<1$ & .4 & $<1$ & $<1$ & $<1$ \\
\hline \multirow[t]{2}{*}{56} & $05-09-97$ & 1 & 64 & 13 & 31 & $<1$ & 4 & 2.5 & $<1$ & 1 & $<1$ \\
\hline & $05-09-97$ & 1 & 86 & 13 & 31 & $<1$ & 4 & 2.6 & - & 1 & $<1$ \\
\hline \multirow[t]{2}{*}{58} & $11-01-96$ & $<1$ & 7 & 5 & 22 & $<1$ & 1 & 1.0 & - & $<1$ & $<1$ \\
\hline & $11-01-96$ & $<1$ & 6 & 5 & 23 & $<1$ & 1 & .9 & -- & $<1$ & $<1$ \\
\hline \multirow[t]{2}{*}{59} & $05-24-97$ & $<1$ & 97 & 5 & 12 & $<1$ & 2 & .5 & - & $<1$ & $<1$ \\
\hline & $05-24-97$ & $<1$ & 86 & 6 & 12 & $<1$ & 2 & .5 & -- & $<1$ & $<1$ \\
\hline \multirow[t]{2}{*}{25} & $02-11-98$ & $<1$ & 3 & 3 & 18 & $<1$ & $<1$ & $<.3$ & - & 1 & $<1$ \\
\hline & $02-11-98$ & $<1$ & 3 & 2 & 19 & $<1$ & $<1$ & $<.3$ & -- & $<1$ & $<1$ \\
\hline \multirow[t]{2}{*}{44} & $05-06-98$ & $<1$ & 12 & 3 & 11 & $<1$ & 1 & 1.1 & -- & $<1$ & $<1$ \\
\hline & $05-06-98$ & $<1$ & 14 & 2 & 10 & $<1$ & 1 & 1.2 & - & $<1$ & $<1$ \\
\hline \multirow[t]{2}{*}{17} & $10-31-96$ & $<1$ & 17 & $<1$ & 26 & $<1$ & 52 & 54 & $<1$ & $<1$ & 17 \\
\hline & $10-31-96$ & $<1$ & 20 & $<1$ & 26 & $<1$ & 53 & 53 & $<1$ & $<1$ & 17 \\
\hline \multirow[t]{2}{*}{8} & 09-13-99 & - & 36 & 32 & -- & - & $<1$ & $<.3$ & - & - & -- \\
\hline & $09-13-99$ & -- & 37 & 32 & -. & -- & $<1$ & $<.3$ & -- & -- & -- \\
\hline
\end{tabular}

\begin{tabular}{|c|c|c|c|c|c|c|c|c|c|c|c|}
\hline $\begin{array}{c}\text { Site } \\
\text { number } \\
\text { (fig. 2) }\end{array}$ & Date & $\begin{array}{l}\text { Copper, } \\
\text { total } \\
\text { recov- } \\
\text { erable } \\
(\mu \mathrm{g} / \mathrm{L} \text { as } \\
\mathrm{Cu})\end{array}$ & $\begin{array}{c}\text { Copper, } \\
\text { dissolved } \\
(\mu \mathrm{g} / \mathrm{L} \text { as } \\
\text { Cu) }\end{array}$ & $\begin{array}{c}\text { Iron, } \\
\text { total } \\
\text { recov- } \\
\text { erable } \\
(\mu \mathrm{g} / \mathrm{L} \text { as } \\
\text { Fe })\end{array}$ & $\begin{array}{c}\text { Iron, } \\
\text { dissolved } \\
(\mu \mathrm{g} / \mathrm{L} \text { as } \\
\text { Fe })\end{array}$ & $\begin{array}{c}\text { Lead, } \\
\text { total } \\
\text { recov- } \\
\text { erable } \\
(\mu \mathrm{g} / \mathrm{L} \text { as } \\
\text { Pb })\end{array}$ & $\begin{array}{c}\text { Lead, } \\
\text { dissolved } \\
(\mu \mathrm{g} / \mathrm{L} \text { as } \\
\text { Pb) }\end{array}$ & $\begin{array}{c}\text { Lithium, } \\
\text { dissolved } \\
(\mu \mathrm{g} / \mathrm{L} \text { as } \\
\mathrm{Li})\end{array}$ & $\begin{array}{c}\text { Manga- } \\
\text { nese, } \\
\text { total } \\
\text { recov- } \\
\text { erable } \\
(\mu \mathrm{g} / \mathrm{L} \text { as } \\
\mathrm{Mn})\end{array}$ & $\begin{array}{l}\text { Manga- } \\
\text { nese, } \\
\text { dissolved } \\
(\mu \mathrm{g} / \mathrm{L} \text { as } \\
\text { Mn) }\end{array}$ & $\begin{array}{c}\text { Mercury, } \\
\text { total } \\
\text { recov- } \\
\text { erable } \\
(\mu \mathrm{g} / \mathrm{L} \text { as } \\
\mathrm{Hg})\end{array}$ \\
\hline \multirow[t]{2}{*}{24} & $12-03-96$ & 3 & 3 & 100 & 47 & $<1$ & $<1$ & $<4$ & 10 & 6 & $<0.1$ \\
\hline & $12-03-96$ & 3 & 3 & 90 & 44 & $<1$ & $<1$ & $<4$ & 10 & 6 & $<.1$ \\
\hline \multirow[t]{2}{*}{56} & $05-09-97$ & 17 & 8 & 1,200 & 62 & 54 & 1.6 & 5 & 590 & 329 & $<.1$ \\
\hline & $05-09-97$ & 22 & 9 & 1,100 & 71 & 88 & 1.8 & -- & 550 & 325 & - \\
\hline \multirow[t]{2}{*}{58} & $11-01-96$ & 10 & 9 & 220 & 91 & 1 & $<1$ & 4 & 50 & 29 & $<.1$ \\
\hline & $11-01-96$ & 12 & 9 & 210 & 81 & 1 & $<1$ & 5 & 50 & 30 & $<.1$ \\
\hline \multirow[t]{2}{*}{59} & $05-24-97$ & 110 & 25 & 6,600 & 130 & 72 & $<1$ & -- & 240 & 17 & $<.1$ \\
\hline & $05-24-97$ & 110 & 24 & 4,000 & 140 & 68 & 1 & -- & 220 & 17 & $<.1$ \\
\hline \multirow[t]{2}{*}{25} & $02-11-98$ & 8 & 6 & 270 & 64 & $<1$ & $<1$ & -- & 19 & 14 & - \\
\hline & $02-11-98$ & 8 & 6 & 270 & 66 & $<1$ & $<1$ & - & 19 & 14 & -- \\
\hline \multirow[t]{2}{*}{44} & $05-06-98$ & 41 & 27 & 660 & 210 & 5 & $<1$ & -- & 55 & 31 & -- \\
\hline & $05-06-98$ & 40 & 27 & 640 & 200 & 5 & $<1$ & -- & 55 & 31 & -- \\
\hline \multirow[t]{2}{*}{17} & $10-31-96$ & 590 & 537 & 2,600 & 570 & 12 & 2.0 & 8 & 1,700 & 1,690 & $<.1$ \\
\hline & $10-31-96$ & 560 & 574 & 2,600 & 580 & 12 & 2.0 & 7 & 1,700 & 1,730 & $<.1$ \\
\hline \multirow[t]{2}{*}{8} & $09-13-99$ & 3 & 2 & 220 & 160 & 5 & 2.0 & -- & 61 & 55 & - \\
\hline & $09-13-99$ & 3 & 2 & 230 & 160 & 6 & 2.0 & -- & 62 & 55 & -- \\
\hline
\end{tabular}


Table 5. Water-quality data for field duplicates, Boulder River watershed, Montana (Continued)

\begin{tabular}{|c|c|c|c|c|c|c|c|c|c|c|}
\hline $\begin{array}{c}\text { Site } \\
\text { number } \\
\text { (fig. 2) }\end{array}$ & Date & $\begin{array}{c}\text { Mercury, } \\
\text { dissolved } \\
(\mu \mathrm{g} / \mathrm{L} \text { as } \\
\mathrm{Hg})\end{array}$ & $\begin{array}{l}\text { Molyb- } \\
\text { denum, } \\
\text { dissolved } \\
(\mu \mathrm{g} / \mathrm{L} \text { as } \\
\text { Mo) }\end{array}$ & $\begin{array}{c}\text { Nickel, } \\
\text { total } \\
\text { recov- } \\
\text { erable } \\
(\mu \mathrm{g} / \mathrm{L} \text { as } \\
\mathrm{Ni})\end{array}$ & $\begin{array}{c}\text { Nickel, } \\
\text { dissolved } \\
(\mu \mathrm{g} / \mathrm{L} \text { as } \\
\mathrm{Ni})\end{array}$ & $\begin{array}{c}\text { Silver, } \\
\text { total } \\
\text { recov- } \\
\text { erable } \\
(\mu \mathrm{g} / \mathrm{L} \text { as } \\
\text { Ag) }\end{array}$ & $\begin{array}{c}\text { Silver, } \\
\text { dissolved } \\
(\mu \mathrm{g} / \mathrm{L} \text { as } \\
\mathrm{Ag})\end{array}$ & $\begin{array}{c}\text { Uranium, } \\
\text { dissolved } \\
(\mu \mathrm{g} / \mathrm{L} \text { as } \mathrm{U})\end{array}$ & $\begin{array}{c}\text { Zinc, } \\
\text { total } \\
\text { recov- } \\
\text { erable } \\
(\mu \mathrm{g} / \mathrm{L} \text { as } \\
\mathrm{Zn})\end{array}$ & $\begin{array}{c}\text { Zinc, } \\
\text { dissolved } \\
(\mu \mathrm{g} / \mathrm{L} \text { as } \\
\mathrm{Zn})\end{array}$ \\
\hline \multirow[t]{2}{*}{24} & $12-03-96$ & $<0.1$ & $<1$ & $<1$ & $<1$ & $<1$ & $<1$ & $<1$ & 90 & 82 \\
\hline & $12-03-96$ & $<.1$ & $<1$ & $<1$ & $<1$ & $<1$ & $<1$ & $<1$ & 90 & 81 \\
\hline \multirow[t]{2}{*}{56} & $05-09-97$ & -- & 1 & 2 & 1 & $<1$ & $<1$ & 4 & 1,100 & 714 \\
\hline & $05-09-97$ & -- & 1 & -- & $<1$ & -- & $<1$ & 4 & 1,100 & 750 \\
\hline \multirow[t]{2}{*}{58} & $11-01-96$ & - & 1 & -- & $<1$ & -. & $<1$ & 2 & 230 & 208 \\
\hline & $11-01-96$ & - & $<1$ & -- & $<1$ & -- & $<1$ & 2 & 230 & 207 \\
\hline \multirow[t]{2}{*}{59} & $05-24-97$ & -- & $<1$ & -- & $<1$ & -- & $<1$ & $<1$ & 250 & 89 \\
\hline & $05-24-97$ & -- & $<1$ & -- & $<1$ & -- & $<1$ & $<1$ & 250 & 86 \\
\hline \multirow[t]{2}{*}{25} & $02-11-98$ & - & $<1$ & -- & $<1$ & -- & $<1$ & 1 & 44 & 44 \\
\hline & $02-11-98$ & -- & $<1$ & -- & $<1$ & - & $<1$ & 1 & 44 & 42 \\
\hline \multirow[t]{2}{*}{44} & $05-06-98$ & - & $<1$ & -- & $<1$ & - & $<1$ & $<1$ & 140 & 120 \\
\hline & $05-06-98$ & -- & $<1$ & -- & $<1$ & - & $<1$ & $<1$ & 140 & 120 \\
\hline \multirow[t]{2}{*}{17} & $10-31-96$ & $<.1$ & $<1$ & 13 & 12 & $<1$ & $<1$ & 4 & 6,000 & 5,660 \\
\hline & $10-31-96$ & $<.1$ & $<1$ & 13 & 12 & $<1$ & $<1$ & 4 & 5,800 & 5,690 \\
\hline \multirow[t]{2}{*}{8} & 09-13-99 & -- & - & -- & - & -- & -- & - & 37 & 35 \\
\hline & 09-13-99 & -- & - & -- & -- & -- & -- & -- & $<31$ & 35 \\
\hline
\end{tabular}


Table 6. Water-quality data for field blanks, Boulder River watershed, Montana

[ANC, acid-neutralizing capacity determined on unfiltered samples, formerly referred to as alkalinity. Abbreviations: lab, laboratory; $\mu \mathrm{g} / \mathrm{L}$, micrograms per liter; $\mu \mathrm{S} / \mathrm{cm}$, microsiemens per centimeter at 25 degrees Celsius; $\mathrm{mg} / \mathrm{L}$, milligrams per liter. Symbols: $<$, less than minimum reporting level; --, no data]

\begin{tabular}{|c|c|c|c|c|c|c|c|c|c|}
\hline Date & Time & $\begin{array}{c}\text { Specific } \\
\text { conduc- } \\
\text { tance, } \\
\text { lab } \\
(\mu \mathrm{S} / \mathrm{cm})\end{array}$ & $\begin{array}{c}\mathrm{pH}, \\
\text { lab } \\
\text { (standard } \\
\text { units) }\end{array}$ & $\begin{array}{c}\text { Calcium } \\
\text { dissolved, } \\
(\mathrm{mg} / \mathrm{L} \text { as } \\
\mathrm{Ca})\end{array}$ & $\begin{array}{c}\text { Magne- } \\
\text { sium, } \\
\text { dissolved } \\
\text { (mg/L as } \mathrm{Mg} \text { ) }\end{array}$ & $\begin{array}{l}\text { Sodium, } \\
\text { dissolved } \\
(\mathrm{mg} / \mathrm{L} \text { as } \mathrm{Na})\end{array}$ & $\begin{array}{l}\text { Potas- } \\
\text { sium, } \\
\text { dissolved } \\
\text { (mg/L as } \\
\text { K) }\end{array}$ & $\begin{array}{c}\mathrm{ANC}, \\
\text { lab } \\
(\mathrm{mg} / \mathrm{L} \text { as } \\
\left.\mathrm{CaCO}_{3}\right)\end{array}$ & $\begin{array}{c}\text { Sulfate, } \\
\text { dissolved } \\
\left(\mathrm{mg} / \mathrm{L} \text { as } \mathrm{SO}_{4}\right)\end{array}$ \\
\hline $10-31-96^{1}$ & 1350 & 1 & 6.1 & $<0.02$ & $<0.01$ & $<0.2$ & $<0.1$ & 1.5 & $<0.1$ \\
\hline $12-04-96^{1}$ & 0945 & 2 & 7.3 & $<.02$ & $<.01$ & $<.2$ & $<.1$ & 1.5 & $<.1$ \\
\hline $02-12-97^{2}$ & 0900 & 2 & 7.9 & $<.02$ & $<.01$ & - & - & - & - \\
\hline $05-22-97^{1}$ & 1245 & 2 & 7.1 & $<.02$ & $<.01$ & $<.2$ & $<.1$ & 1.1 & $<.1$ \\
\hline $06-18-97^{2}$ & 1430 & 2 & 7.9 & $<.02$ & $<.01$ & $<.2$ & $<.1$ & 1.8 & $<.1$ \\
\hline $09-22-97^{2}$ & 1500 & 2 & 7.0 & $<.02$ & $<.01$ & $<.2$ & $<.1$ & 1.7 & .1 \\
\hline $09-22-97^{1}$ & 1515 & 2 & 7.0 & - & - & - & -- & - & -- \\
\hline $05-05-98^{3}$ & 1030 & 1 & 7.7 & $<.02$ & $<.004$ & $<.1$ & $<.1$ & 1.5 & $<.1$ \\
\hline $09-25-98^{3}$ & 0945 & 2 & 7.7 & $<.02$ & $<.004$ & $<.1$ & $<.1$ & 1.4 & $<.1$ \\
\hline $05-28-99^{3}$ & 0825 & 1 & 7.1 & $<.02$ & $<.004$ & $<.06$ & $<.1$ & 1.9 & $<.1$ \\
\hline $05-28-99^{3}$ & 1000 & 2 & 7.3 & $<.02$ & .008 & $<.06$ & $<.1$ & 1.7 & $<.1$ \\
\hline $08-05-99^{3}$ & 1145 & 2 & 5.5 & $<.02$ & $<.12$ & $<.09$ & $<.1$ & 1.8 & $<3$ \\
\hline
\end{tabular}

\begin{tabular}{|c|c|c|c|c|c|c|c|c|c|c|}
\hline Date & $\begin{array}{c}\text { Chloride, } \\
\text { dissolved } \\
\text { (mg/L as Cl) }\end{array}$ & $\begin{array}{c}\text { Fluoride, } \\
\text { dissolved } \\
\text { (mg/L as } \\
\text { F) }\end{array}$ & $\begin{array}{c}\text { Silica, } \\
\text { dissolved } \\
(\mathrm{mg} / \mathrm{L} \text { as } \\
\left.\mathrm{SiO}_{2}\right)\end{array}$ & $\begin{array}{c}\text { Nitrite, } \\
\text { dissolved } \\
\text { (mg/L as } \\
\mathrm{N})\end{array}$ & $\begin{array}{l}\text { Nitrite pius } \\
\text { nitrate, } \\
\text { dissolved } \\
\text { (mg/L as } \\
\text { N) }\end{array}$ & $\begin{array}{c}\text { Ammonia, } \\
\text { dissolved } \\
\text { (mg/L as } N)\end{array}$ & $\begin{array}{c}\text { Ammonia } \\
\text { plus organic } \\
\text { nitrogen, } \\
\text { total } \\
\text { (mg/L as } \\
\mathrm{N})\end{array}$ & $\begin{array}{l}\text { Phos- } \\
\text { phorus, } \\
\text { total } \\
\text { (mg/L as } \\
\text { P) }\end{array}$ & $\begin{array}{l}\text { Phos- } \\
\text { phorus, } \\
\text { ortho, } \\
\text { dissolved } \\
\text { (mg/L as } \\
\text { P) }\end{array}$ & $\begin{array}{c}\text { Alum- } \\
\text { inum, } \\
\text { total } \\
\text { recov- } \\
\text { erable } \\
(\mu \mathrm{g} / \mathrm{L} \text { as } \\
\text { Al) }\end{array}$ \\
\hline $10-31-96$ & $<0.1$ & $<0.1$ & $<0.1$ & - & -- & - & -. & -- & - & -- \\
\hline $12-04-96$ & $<.1$ & $<.1$ & $<.1$ & $<0.01$ & $<0.05$ & 0.03 & -- & -- & $<0.01$ & -- \\
\hline $02-12-97$ & -- & -- & -- & - & -- & -- & -- & - & -- & -- \\
\hline $05-22-97$ & .1 & $<.1$ & .2 & -- & -- & -- & -- & -- & -- & -- \\
\hline $06-18-97$ & $<.1$ & $<.1$ & .1 & -- & -- & -- & -- & - & -- & -- \\
\hline $09-22-97$ & $<.1$ & $<.1$ & .4 & $<.01$ & $<.05$ & $<.01$ & $<.2$ & $<.01$ & $<.01$ & $<10$ \\
\hline $09-22-97$ & -- & - & -- & -- & -- & -- & - & -- & -- & -- \\
\hline $05-05-98$ & $<.1$ & $<.1$ & $<.1$ & -- & -- & -- & -- & -- & - & $<10$ \\
\hline $09-25-98$ & $<.1$ & $<.1$ & $<.1$ & -- & - & -- & -- & $<.05$ & -- & $<10$ \\
\hline $05-28-99$ & $<.1$ & -- & -- & - & -- & -- & -- & -- & -- & $<28$ \\
\hline $05-28-99$ & $<.1$ & -- & -- & -- & -- & - & -- & -- & -- & $<28$ \\
\hline 08-05-99 & $<.3$ & -- & -- & -- & - & -- & -- & -- & -- & $<28$ \\
\hline
\end{tabular}


Table 6. Water-quality data for field blanks, Boulder River watershed, Montana (Continued)

\begin{tabular}{|c|c|c|c|c|c|c|c|c|c|c|}
\hline Date & $\begin{array}{c}\text { Alum- } \\
\text { inum, } \\
\text { dissolved } \\
(\mu \mathrm{g} / \mathrm{L} \text { as } \mathrm{Al})\end{array}$ & $\begin{array}{c}\text { Antimony, } \\
\text { dissolved } \\
(\mu \mathrm{g} / \mathrm{L} \text { as } \\
\text { Sb) }\end{array}$ & $\begin{array}{c}\text { Arsenic, } \\
\text { total } \\
\text { recov- } \\
\text { erable } \\
(\mu \mathrm{g} / \mathrm{L} \text { as } \\
\text { As) }\end{array}$ & $\begin{array}{c}\text { Arsenic, } \\
\text { dissolved } \\
(\mu \mathrm{g} / \mathrm{L} \text { as } \\
\text { As })\end{array}$ & $\begin{array}{c}\text { Barium, } \\
\text { dissolved } \\
(\mu \mathrm{g} / \mathrm{L} \text { as } \\
\mathrm{Ba})\end{array}$ & $\begin{array}{c}\text { Beryllium, } \\
\text { dissolved, } \\
(\mu \mathrm{g} / \mathrm{L} \mathrm{a} \\
\mathrm{Be})\end{array}$ & $\begin{array}{c}\text { Cadmium, } \\
\text { total } \\
\text { recov- } \\
\text { erable } \\
(\mu \mathrm{g} / \mathrm{L} \text { as } \\
\text { Cd })\end{array}$ & $\begin{array}{c}\text { Cadmium, } \\
\text { dissolved } \\
(\mu \mathrm{g} / \mathrm{L} \text { as } \\
\text { Cd })\end{array}$ & $\begin{array}{c}\text { Chromium, } \\
\text { total } \\
\text { recov- } \\
\text { erable } \\
(\mu \mathrm{g} / \mathrm{L} \text { as } \\
\text { Cr) }\end{array}$ & $\begin{array}{c}\text { Chromium, } \\
\text { dissolved } \\
(\mu \mathrm{g} / \mathrm{L} \text { as } \\
\text { Cr) }\end{array}$ \\
\hline $10-31-96$ & 3 & $<1$ & $<1$ & $<1$ & $<1$ & $<1$ & $<1$ & $<1$ & $<1$ & $<1$ \\
\hline $12-04-96$ & 4 & $<1$ & $<1$ & $<1$ & $<1$ & $<1$ & $<1$ & $<1$ & $<1$ & $<1$ \\
\hline $02-12-97$ & $<1$ & $<1$ & $<1$ & $<1$ & $<1$ & $<1$ & $<1$ & $<.1$ & -- & $<1$ \\
\hline $05-22-97$ & 4 & $<1$ & -- & $<1$ & $<1$ & $<1$ & $<1$ & $<.1$ & $<1$ & $<1$ \\
\hline $06-18-97$ & 4 & $<1$ & - & $<1$ & $<1$ & $<1$ & $<1$ & $<.1$ & -- & $<1$ \\
\hline $09-22-97$ & $<1$ & $<1$ & $<1$ & $<1$ & $<1$ & $<1$ & $<1$ & $<.1$ & -- & $<1$ \\
\hline $09-22-97$ & 3 & $<1$ & - & -- & $<1$ & $<1$ & -- & $<.1$ & - & $<1$ \\
\hline $05-05-98$ & $<1$ & $<1$ & $<1$ & $<1$ & $<1$ & $<1$ & $<1$ & $<.3$ & - & $<1$ \\
\hline $09-25-98$ & 2 & $<1$ & $<1$ & $<1$ & $<1$ & $<1$ & $<1$ & $<.3$ & - & $<1$ \\
\hline $05-28-99$ & $<1$ & - & $<1$ & $<1$ & -- & -- & $<1$ & $<.3$ & -- & -- \\
\hline $05-28-99$ & $<1$ & -- & $<1$ & $<1$ & - & - & $<1$ & $<.3$ & - & - \\
\hline $08-05-99$ & $<1$ & - & $<1$ & $<1$ & -- & -- & $<1$ & $<.3$ & - & -- \\
\hline
\end{tabular}

\begin{tabular}{|c|c|c|c|c|c|c|c|c|c|c|}
\hline Date & $\begin{array}{c}\text { Cobalt, } \\
\text { dissolved } \\
(\mu \mathrm{g} / \mathrm{L} \text { as } \\
\text { Co })\end{array}$ & $\begin{array}{l}\text { Copper, total } \\
\text { recov- } \\
\text { erable } \\
(\mu \mathrm{g} / \mathrm{L} \text { as } \\
\mathrm{Cu})\end{array}$ & $\begin{array}{c}\text { Copper, } \\
\text { dissolved } \\
(\mu \mathrm{g} / \mathrm{L} \text { as } \\
\mathrm{Cu})\end{array}$ & $\begin{array}{c}\text { Iron, } \\
\text { total } \\
\text { recov- } \\
\text { erable } \\
(\mu \mathrm{g} / \mathrm{L} \text { as } \\
\mathrm{Fe})\end{array}$ & $\begin{array}{c}\text { Iron, } \\
\text { dissolved } \\
(\mu \mathrm{g} / \mathrm{L} \text { as } \\
\mathrm{Fe})\end{array}$ & $\begin{array}{c}\text { Lead, } \\
\text { total } \\
\text { recov- } \\
\text { erable } \\
(\mu \mathrm{g} / \mathrm{L} \text { as } \\
\mathrm{Pb})\end{array}$ & $\begin{array}{c}\text { Lead, } \\
\text { dissolved } \\
(\mu \mathrm{g} / \mathrm{L} \text { as } \\
\text { Pb) }\end{array}$ & $\begin{array}{c}\text { Lithium, } \\
\text { dissolved } \\
(\mu \mathrm{g} / \mathrm{L} \text { as } \\
\text { Li) }\end{array}$ & $\begin{array}{c}\text { Manganese, } \\
\text { total } \\
\text { recov- } \\
\text { erable } \\
(\mu \mathrm{g} / \mathrm{L} \text { as } \mathrm{Mn})\end{array}$ & $\begin{array}{c}\text { Manga- } \\
\text { nese, } \\
\text { dissolved } \\
(\mu \mathrm{g} / \mathrm{L} \text { as } \mathrm{Mn})\end{array}$ \\
\hline $10-31-96$ & $<1$ & $<1$ & $<1$ & $<10$ & $<3$ & $<1$ & $<1$ & $<4$ & $<10$ & $<1$ \\
\hline $12-04-96$ & $<1$ & $<1$ & $<1$ & $<10$ & $<3$ & $<1$ & $<1$ & $<4$ & $<10$ & $<1$ \\
\hline $02-12-97$ & $<1$ & $<1$ & $<1$ & $<10$ & $<3$ & $<1$ & $<1$ & - & $<10$ & $<1$ \\
\hline $05-22-97$ & $<1$ & $<1$ & $<1$ & $<10$ & $<3$ & $<1$ & $<1$ & $<4$ & $<10$ & $<1$ \\
\hline $06-18-97$ & $<1$ & $<1$ & $<1$ & $<10$ & $<3$ & $<1$ & $<1$ & - & $<10$ & $<1$ \\
\hline $09-22-97$ & $<1$ & $<1$ & $<1$ & $<10$ & 3 & $<1$ & $<1$ & - & $<10$ & $<1$ \\
\hline $09-22-97$ & $<1$ & -- & $<1$ & -- & - & - & $<1$ & -- & - & $<1$ \\
\hline $05-05-98$ & $<1$ & $<1$ & $<1$ & $<10$ & $<10$ & $<1$ & $<1$ & - & $<10$ & $<1$ \\
\hline $09-25-98$ & $<1$ & $<1$ & $<1$ & $<10$ & $<10$ & $<1$ & $<1$ & - & $<10$ & $<1$ \\
\hline $05-28-99$ & - & $<1$ & $<1$ & $<14$ & $<10$ & $<1$ & $<1$ & - & $<3$ & $<1$ \\
\hline $05-28-99$ & - & $<1$ & $<1$ & $<14$ & $<10$ & $<1$ & $<1$ & - & $<3$ & $<1$ \\
\hline $08-05-99$ & -- & $<1$ & $<1$ & $<14$ & $<10$ & $<1$ & $<1$ & - & $<3$ & $<1$ \\
\hline
\end{tabular}


Table 6. Water-quality data for field blanks, Boulder River watershed, Montana (Continued)

\begin{tabular}{|c|c|c|c|c|c|c|c|c|c|c|}
\hline Date & $\begin{array}{c}\text { Mercury, } \\
\text { total } \\
\text { recov- } \\
\text { erable } \\
(\mu \mathrm{g} / \mathrm{L} \text { as } \\
\text { Hg) }\end{array}$ & $\begin{array}{c}\text { Mercury, } \\
\text { dissolved } \\
(\mu \mathrm{g} / \mathrm{L} \text { as } \\
\mathrm{Hg})\end{array}$ & $\begin{array}{c}\text { Molyb- } \\
\text { denum, } \\
\text { dissolved } \\
(\mu \mathrm{g} / \mathrm{L} \text { as } \\
\text { Mo) }\end{array}$ & $\begin{array}{c}\text { Nickel, } \\
\text { total } \\
\text { recov- } \\
\text { erable } \\
(\mu \mathrm{g} / \mathrm{L} \text { as } \\
\mathrm{Ni})\end{array}$ & $\begin{array}{l}\text { Nickel, } \\
\text { dissolved } \\
(\mu \mathrm{g} / \mathrm{L} \text { as } \\
\mathrm{Ni})\end{array}$ & $\begin{array}{c}\text { Silver, } \\
\text { total } \\
\text { recov- } \\
\text { erable } \\
(\mu \mathrm{g} / \mathrm{L} \text { as } \\
\text { Ag) }\end{array}$ & $\begin{array}{c}\text { Silver, } \\
\text { dissolved } \\
(\mu \mathrm{g} / \mathrm{L} \text { as } \\
\mathrm{Ag})\end{array}$ & $\begin{array}{c}\text { Uranium, } \\
\text { dissolved } \\
(\mu \mathrm{g} / \mathrm{L} \text { as } \\
\text { U) }\end{array}$ & $\begin{array}{c}\text { Zinc, } \\
\text { total } \\
\text { recov- } \\
\text { erable } \\
(\mu \mathrm{g} / \mathrm{L} \text { as } \\
\text { Zn) }\end{array}$ & $\begin{array}{c}\text { Zinc, } \\
\text { dissolved } \\
(\mu \mathrm{g} / \mathrm{L} \text { as } \\
\mathrm{Zn})\end{array}$ \\
\hline $10-31-96$ & $<0.1$ & $<0.1$ & $<1$ & $<1$ & $<1$ & $<1$ & $<1$ & $<1$ & $<10$ & $<1$ \\
\hline $12-04-96$ & $<.1$ & $<.1$ & $<1$ & $<1$ & $<1$ & $<1$ & $<1$ & $<1$ & $<10$ & $<1$ \\
\hline $02-12-97$ & - & - & $<1$ & - & $<1$ & - & $<1$ & $<1$ & $<10$ & $<1$ \\
\hline $05-22-97$ & $<.1$ & -- & $<1$ & $<1$ & $<1$ & $<1$ & $<1$ & $<1$ & $<10$ & $<1$ \\
\hline $06-18-97$ & - & - & $<1$ & -- & $<1$ & - & $<1$ & $<1$ & $<10$ & 1 \\
\hline $09-22-97$ & - & -- & $<1$ & -- & $<1$ & -- & $<1$ & $<1$ & $<10$ & $<1$ \\
\hline $09-22-97$ & -- & - & $<1$ & -- & $<1$ & -- & $<1$ & $<1$ & - & $<1$ \\
\hline $05-05-98$ & - & - & $<1$ & - & $<1$ & -- & $<1$ & $<1$ & $<10$ & $<1$ \\
\hline $09-25-98$ & -- & -- & $<1$ & -- & $<1$ & -- & $<1$ & $<1$ & $<10$ & $<1$ \\
\hline $05-28-99$ & $\cdots$ & - & - & -- & -- & -- & -- & -- & $<40$ & $<1$ \\
\hline $05-28-99$ & -- & -- & -- & -- & -- & -- & -- & -- & $<40$ & $<1$ \\
\hline $08-05-99$ & -- & -- & -. & - & -- & -- & - & -- & $<40$ & $<1$ \\
\hline
\end{tabular}

${ }^{1}$ Blank preserved in the field with concentrated nitric acid from glass vials.

${ }^{2}$ Blank preserved in the field with concentrated nitric acid from polytetrafluorethylene vials.

${ }^{3}$ Blank preserved in the laboratory with concentrated nitric acid from pipettor within 24 hours of sample collection. 